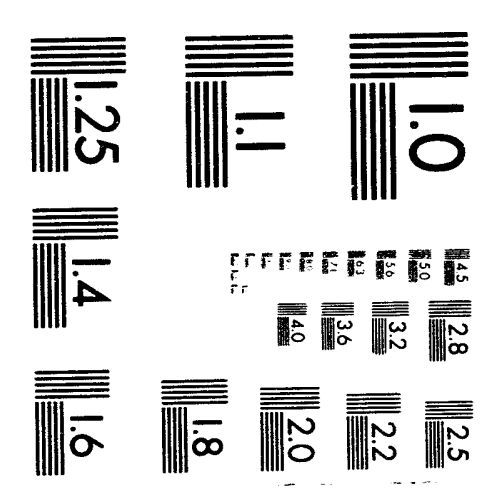

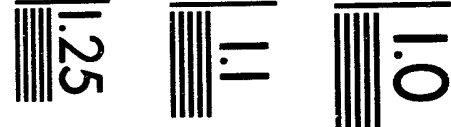

Everneser

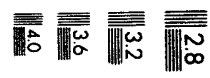

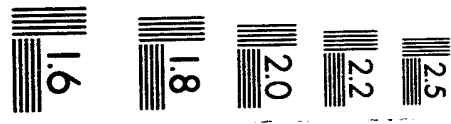



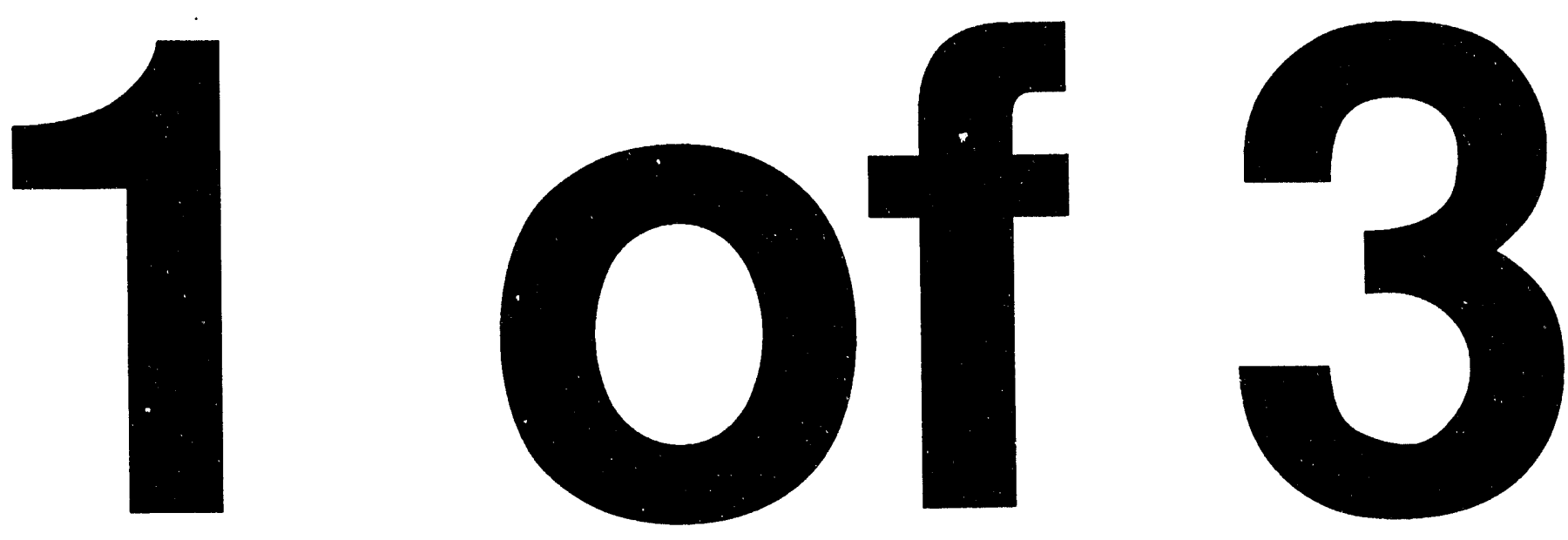


\title{
New York Btate
}

Energy Research and Development Authority

Two Rockefelier Plaza - Albany, New York 12223

(518) 465-6251

\section{An Energy Authority Report in Brief}

\author{
Report: Uncertainty Analysis of the Measured Performance \\ Rating (MPR) Method, Report 94-2
}

Project Manager: Norine H. Karins

Contractor:

\author{
NAHB/National Research Center \\ Upper Marlboro, MD
}

Background: The Measured Performance Rating (MPR) method is an empirical approach to evaluating building thermal performance based on one or more days of testing. Empirical data are collected on site during an actual heating period, from which an estimate of building heat loss coefficient, heating system efficiency and annual energy consumption are determined. As with all quantitative methods, accuracy of an estimate is affected by computational methodologies and quality of data. Furthermore, the overall uncertainty places limitation on the ability to detect weatherization treatments. The detection limit was previously unknown and it is an important characteristic for defining the applicability of the test method.

Objectives: To determine the uncertainty of the estimates made by the MPR system using four approaches: 1) comparison of predicted fuel consumption based on MPR results to utility bill data on four homes; 2 ) analysis of repeated MPR tests on actual homes; 3 ) many repeated tests on two simulated houses under varying weather conditions and thermal parameter changes; and 4) evaluation of measurement equipment bias and scatter that are propagated into the heat loss coefficient and the heating system efficiency estimates.

R \& D Results: The analysis indicates that the MPR method should be able to detect as little as a 7 percent change in the heat loss coefficient at the 95 percent confidence level. MPR appears sufficiently robust for characterizing common weatherization treatments, i.e., increasing attic insulation from R-7 to R-19 in a typical, single-story, 1,100-square-foot house, resulting in a 19 percent reduction in heat loss coefficient. Furnace efficiency uncertainties ranged up to three times those of the heat loss coefficient. Measurement uncertainties (at the 95 percent confidence level) were estimated to be from 1 to 5 percent for heat loss coefficients, and 1.5 percent for a typical furnace efficiency.

The analysis also shows a limitation in applying MPR to houses with heating ducts in slabs on grade, and to those with very large thermal mass. Most of the uncertainties encountered in the study were due more to the methods of estimating the "true" heat loss coefficients, furnace efficiency, and furnace fuel consumption (by collecting fuel bills and simulating two actual houses) than to the MPR approach. These uncertainties in the true parameter values become evidence for arguments in favor of the need for empirical measures of heat loss coefficient and furnace efficiency, like the MPR method.

Copies Available: A limited number of copies of the full report are available from Media Relations and Outreach, The New York State Energy Research and Development Authority, Two Rockefeller Plaza, Albany, New York 12223. 


\title{
UNCERTAINTY ANALYSIS OF THE MEASURED
}

PERFORMANCE RATING METHOD

\author{
Final Report
}

Prepared for

THE NEW YORK STATE

ENERGY RESEARCH AND DEVELOPMENT AUTHORITY

Project Manager

Norine H. Karins

and

ELECTRIC POWER RESEARCH INSTITUTE

Project Manager

John Kesselring

Prepared by

NAHB RESEARCH CENTER, INC.

400 Prince George's Boulevard

Upper Marlboro, MD 20772-8731

1129-EEED-AEP-89

Energy Authority

Report 94-2

November 1993

8 


\section{NOTICE}

This report was prepared by NAHB Research Center, Inc. (Research Center) in the course of performing work contracted for and sponsored by the Electric Power Research Institute and the New York State Energy Research and Development Authority (hereafter the "Sponsors"). The opinions expressed in this report do not necessarily reflect those of the Sponsors or the State of New York, and reference to any specific product, service, process, or method does not constitute an implied or expressed recommendation or endorsement of it. Further, the Sponsors and the State of New York make no warranties or representations, expressed or implied, as to the fitness for particular purpose or merchantability of any product, apparatus, or service, or the usefulness, completeness, or accuracy of any processes, methods, or other information contained, described, disclosed, or referred to in this report. The Sponsors, the State of New York, and the contractor make no representation that the use of any product, apparatus, process, method, or other information will not infringe privately owned rights and will assume no liability for any loss, injury, or damage resulting from, or occurring in connection with, the use of information contained, described, disclosed, or referred to in this report.

First Printing: February 1994 


\begin{abstract}
A report was commissioned by the New York State Energy Research and Development Authority and the Electric Power Research Institute to evaluate the uncertainties in the energy monitoring method known as measured performance rating (MPR). The work is intended to help further development of the MPR system by quantitatively analyzing the uncertainties in estimates of the heat loss coefficients and heating system efficiencies. The analysis indicates that the MPR should be able to detect as little as a 7 percent change in the heat loss coefficient at 95 percent confidence level. MPR appears sufficiently robust for characterizing common weatherization treatments; e.g., increasing attic insulation from R-7 to R-19 in a typical single-story, 1,100 sq. $\mathrm{ft}$. house resulting in a 19 percent reduction in heat loss coefficient. Furnace efficiency uncertainties ranged up to three times those of the heat loss coefficients. Measurement uncertainties (at the 95 percent confidence level) were estimated to be from 1 to 5 percent for heat loss coefficients and 1.5 percent for a typical furnace efficiency. The analysis also shows a limitation in applying MPR to houses with heating ducts in slabs on grade and to those with very large thermal mass. Most of the uncertainties encountered in the study were due more to the methods of estimating the "true" heat loss coefficients, furnace efficiency, and furnace fuel consumption (by collecting fuel bills and simulating two actual houses) than to the MPR approach. These uncertainties in the true parameter values become evidence for arguments in favor of the need of empirical measures of heat loss coefficient and furnace efficiency, like the MPR method, rather than arguments against.
\end{abstract}

Key Words: Short-term energy monitoring, measured performance rating system, uncertainty analysis, residential 


\section{TABLE OF CONTENTS}

$\underline{\text { Section }}$

Page

SUMMARY

1 INTRODUCTION

Background of the MPR Methodology

$1-1$

Background on Uncertainty Analysis

$1-3$

Uncertainty Analysis of the MPR System

2 MEASURED FUEL CONSUMPTION COMPARED TO MPR PREDICTIONS

Results of Two Weatherized Homes

Results of Two Previously Tested Occupied Homes

3 SENSITIVITY ANALYSIS OF TWO PREVIOUSLY TESTED HOMES

Results of Heating Season Simulations

Results of Multi-Parameter Analysis

4 IMPLICATIONS OF THE UNCERTAINTY ANALYSIS RESULTS

5 RECOMMENDATIONS FOR FUTURE WORK

REFERENCES

$\mathrm{R}-1$

APPENDIX A Measurement Uncertainty in the Heat Loss Coefficient and Heating System Efficiency Estimates

APPENDIX B Summary of Predicted Fuel Usage

B-1

APPENDIX C Synertech Systems Corporation Final Report:

Uncertainty Analysis of the Measured Performance

Rating System

C-1

APPENDIX D J.J. Duffy Associates Final Report: Uncertainty

Analysis of the Measured Performance Rating System

D-1 


\section{SUMMARY}

Researchers have explored the use of short-term automated techniques to measure thermal performance of homes. These techniques can help determine the impact and cost-effectiveness of energy conservation measures. This document investigates the uncertainties in estimates of the heat loss coefficient and heating system efficiency made by the MPR method.

BACKGROUND - The MPR method is an empirical approach to evaluating building thermal performance based on one or more days of testing. Empirical data are collected on site during an actual heating period, from which an estimate of building heat loss coefficient, heating system efficiency and annual energy consumption are determined. As with all quantitative methods, accuracy of an estimate is effected by computational methodologies and quality of data. And, the overall uncertainty places limitation on the ability to detect weatherization treatments. The detection limit was previously unknown and it is an important characteristic for defining the applicability of the test method.

OBJECTIVE - To determine the uncertainty of the estimates made by the MPR system.

APPROACH - Four approaches were used in the uncertainty analysis: 1) comparison of predicted fuel consumption based on MPR results to utility bill data on four houses; 2 ) analysis of repeated MPR tests on actual houses; 3) many repeated tests on two simulated houses under varying weather conditions and thermal parameter changes; and 4) evaluation of measurement equipment bias and scatter that are propagated into the heat loss coefficient and the heating system efficiency estimates.

RESULTS - The analysis indicates that the MPR should be able to detect as little as a 7 percent change in the heat loss coefficient at 95 percent confidence level. MPR appears sufficiently robust for characterizing common weatherization treatments; e.g., increasing attic insulation from $\mathrm{R}-7$ to $\mathrm{R}-19$ in a typical single-story, $1,100 \mathrm{sq}$. $\mathrm{ft}$. house resulting in a 19 percent reduction in heat loss coefficient. Furnace efficiency uncertainties ranged up to three times those of the heat loss coefficients. Measurement uncertainties (at the 95 percent confidence level) were estimated to be from 1 to 5 percent for heat loss coefficients and 1.5 percent for a typical furnace efficiency. The analysis also shows a limitation in applying MPR to houses with heating ducts in slabs on grade and to those with very large thermal mass. Most of the uncertainties encountered in the study were due more to the methods of estimating the "true" heat loss coefficients, furnace efficiency, and furnace fuel consumption (by collecting fuel bills and simulating two actual houses) than to the MPR approach. These uncertainties in the true parameter values become evidence for arguments in favor of the need of empirical measures of heat loss coefficient and furnace efficiency, like the MPR method, rather than arguments against. 


\section{IN'TRODUCTION}

The NAHB Research Center, İnc. (Research Center), in conjunction with John Duffy of the University of Massachusetts Lowell and J.J. Duffy Associates, and Synertech Systems Corporation have field tested and developed a short-term energy monitoring method known as the measured performance rating (MPR) system. The MPR system is an empirically-based approach to estinnate a building heat loss coefficient, overall heating system efficiency, and fuel consumption. This technology has potential for aiding utilities and state agencies in evaluating effectiveness of demand-side management (DSM) projects and energy savings attributable to weatherization programs.

Previous work for the New York State Energy Research and Development Authority (the Energy Authority) (Research Center 1991) and the Electric Power Research Institute (EPRI) (Research Center 1992) has shown that uncertainties exist in the estimates of the heat loss coefficient and heating system efficiency. These uncertainties need to be addressed if the MPR system is to become a useful evaluation tool.

The purpose of this phase in the development of the MPR system is to analyze quantitatively the uncertainties in estimates of the heat loss coefficients and heating system efficiencies. The work reported herein was conducted under contract to the Energy Authority and EPRI. J.J. Duffy Associates (Duffy) acted as principal investigator in the sensitivity analysis of heat loss coefficient and heating system efficiency estimates and was assisted by the Research Center. Synertech Systems Corporation (Synertech) assisted the Research Center in comparing utility bill data and MPR predictions of three previously tested homes in the State of New York. In all, four comparisons of actual fuel consumption data to MPR predictions were made: two homes that had repeated tests performed on them and two homes that were weatherized.

\section{BACKGROUND OF THE MPR METHODOLOGY}

Originally, the MPR system was designed by the building industry for use on large samples of buildings; therefore, cost, speed, simplicity, and accuracy were important. The 
method has been in development over several years. Early on, Duffy and Puri (1985) addressed the feasibility of extrapolating short-term test results to long-term predictions. Building on that work, Duffy and Saunders (1987) developed a low-cost approach to short-term energy monitoring for evaluating the energy performance of buildings and successfully field tested it on three homes.

More recently, the method was field tested to determine its practicality in evaluating weatherization measures performed on homes (Research Center 1991), and was also compared to another short-term energy monitoring method (Research Center 1992). During that period of development, Bourque and Duffy (1992) used a system identification approach to refine the MPR analysis to offer explicit uncertainty estimates and flexibility in the number and type of thermal parameters estimated.

The intended use of the MPR system is both to predict fuel consumption of new homes and to measure the effectiveness of weatherization measures on retrofitted dwellings. Actual data are collected onsite over one night of heating and are then used to estimate average seasonal thermal characteristics of the building. Four tests are primarily involved: electric coheating, heating system efficiency, tracer gas dilution, and blower door leakage. A fifth test, the cool-down, is called for when the inside temperature drifts more than $2^{\circ} \mathrm{F}$.

In the electric coheat test, the indoor temperature is maintained at a preset temperature for the duration of the test using portable electric heaters. The measured electric energy consumed during the test period represents the total energy needed to heat the house at the existing conditions. The assumption is made that 100 percent of indoor appliance electricity is converted to heat, so appliance usage is included in the measurement of electric energy consumed during the test. Data collected on electric energy consumption, indoor temperatures, and outdoor temperature are used to calculate an overall unadjusted heat loss coefficient (UA).

At the start of the heating system efficiency test, the portable electric heaters are turned off and the indoor temperature is maintained by the house heating system. The test is 
performed so that at least four complete heating system on-off cycles occur. For typical systems, such as central forced-air systems, this will require only three to four hours. The heat load of the house during the test is calculated from the unadjusted UA determined by the coheat test, and the temperature conditions at the time of the heating system efficiency test. The net heat added to the space by the house heating system is computed as the difference between the calculated heating load and measured total electric energy. The efficiency is then estimated by the ratio of net heat added to the heating system fuel flow rate and on-time.

Because a significant component of the UA is believed to be due to air infiltration, a tracer gas test is performed during the coheat test to assess the air infiltration affect on the coheat test results. This test includes injecting a tracer gas into the interior of the house and mixing it by fan for at least 30 minutes. Air samples are then collected every 30 minutes until at least four samples have been collected. The samples are then analyzed for concentration of tracer gas. The dilution of tracer gas concentration over time is an indicator of the air infiltration rate if the house air is assumed to be well mixed. The UA due to infiltration during the coheat test can then be calculated from the tracer gas test results.

To obtain the overall adjusted heating scason UA, the heating season average UA due to infiltration needs to be estimated. Wind speed is measured onsite during the night of testing, and a blower door air leakage test is performed the following morning. A model, such as the one developed by Sherman and Grimsrud (1982), is calibrated with empirical data to estimate the heating season average UA due to infiltration. Infiltration models are known to have a bias and, therefore, require compensation to improve accuracy. The MPR protocol accounts for this shortcoming by calibrating the model with a ratio factor of measured air infiltration based on tracer gas dilution measurements and the model's estimates. The tracer gas test is conducted during the coheat test and an infiltration estimate evaluated for those ambient conditions. The overall heating season UA is then estimated by adding the adjusted infiltration UA to the conductive UA determined by the coheat and tracer gas tests. 
gains and weather data to predict either the fuel consumption over a typical heating season or the fuel consumption over a reference period.

The MPR system approach has many strengths: low cost with particular emphasis on low labor costs; measurement-based, not simulation-based; predictable accuracy, preferably within \pm 10 percent; ${ }^{2}$ easy to learn and understand; relatively quick (one day for testing and results); and potential to be insensitive to test operator influences. On the other hand, previous work (Research Center 1991, 1992) has pointed out weaknesses in the MPR approach: consistent bias in the estimates of heat loss coefficients and heating system efficiencies; potential for operator error when manually collecting tracer gas samples; and potential for significant occupant effects if homes are occupieci during testing.

Two alternatives have been suggested for two of these weaknesses: the operator error in manual tracer gas tests can be eliminated with an automated tracer gas test developed by J.J. Duffy Associates, and occupant effects can be eliminated by relocating occupants to alternative locations during testing. The bias (and scatter) in predictions of heat loss coefficients and heating system efficiencies is the subject of this report.

\section{BACKGROUND ON UNCERTAINTY ANALYSIS}

Recent work (Duffy and Saunders 1987, Research Center 1991, Bourque and Duffy 1992) have shown that uncertainties exist in MPR estimates of the heat loss coefficients and heating system efficiencies. These uncertainties need to be addressed if the MPR system is to become a useful evaluation tool.

The MPR system has been designed to be economically accurate, which is an effect of the lowcost, speed and simplicity design constraints. The word accuracy refers to the closeness of agreement between a predicted value and the true value. The degree of inaccuracy is the

\footnotetext{
'Full costs for measurernent and analysis per house may range from $\$ 1,000$ to $\$ 2,000$. Higher costs are expected in occupied homes where greater care to installation and safeguards are required.

${ }^{2}$ Recent MPR predictions of space heating fuel use in four unoccupied homes, two of which were research homes with long-term monitoring, were within a range of 2 to 6 percent of the measured values (Research Center 1991, 1992).
} 
The MPR system has been designed to be economically accurate, which is an effect of the low-cost, speed and simplicity design constraints. The word accuracy refers to the closeness of agreement between a predicted value and the true value. The degree of inaccuracy is the difference between the predicted value and the true value. This total error is the sum of the bias error and the precision error.

The bias error is a systematic, or constant error, during a given test. The bias can not be defined with a statistical equation. Instead, it must be estimated, which can be difficult since the true value is not known. Bias errors, or noise, can occur because of data acquisition including environmental and installation effects as well as the acquisition, conditioning, and storage of sensor outputs. Corrections can be made for bias errors whose values are known, such as those determined by calibration. However, the bias associated with the standard or reference used in the calibration -- no reference or standard is perfect -- will remain in the output of the calibrated device. A bias error source can also be due to data reduction methods, including those in curve-fits and model simplifications. All unknown bias errors need to be estimated.

The precision error is the random component of the total error. It can be determined by taking repeated measurements and calculating the sample standard deviation of the mean. The precision error is sometimes called the repeatability or scatter. Both precision and bias errors are propagated into the derived result; in this example, through the functional relationship between the estimates of the heat loss coefficient and heating system efficiency and their key parameters.

\section{Uncertainty Analysis of the MPR System}

Two approaches were used in the uncertainty analysis: comparison of measured fuel consumption data to MPR predictions for four previously tested homes (two which had repeated tests performed on them and two which were weatherized), and computer simulation tests of models on two previously tested homes. 
The first approach in the uncertainty analysis was to collect and compare measured fuel consumption data to MPR predictions of fuel use for four previously tested homes. The measured fuel consumption data were collected by an elapsed time meter in one home (Winchester home) and through the local utility company for the other three homes. All predictions were made without knowledge of the actual fuel consumption. The results of these comparisons are presented in Section 2.

In the second approach, simulation models based on an electric circuit analogy (a conceptual approach known as a thermal network that allows use of circuit theory to model the heat transfer of a building) were developed to characterize two homes that were repeatedly tested in previous work (Research Center 1991). The models were used to estimate the sensitivity of the heat loss coefficient and heating system efficiency estimates to several key parameters: thermal mass, outdoor temperature, wind speed, solar gain, basement and slab heat loss, and air infiltration.

The models were calibrated using actual MPR data from tests conducted in 1990. The models were then used in repeated simulations of MPR tests under two sets of conditions. The first set contained an entire average heating season of hourly weather data. MPR tests were simulated each night for 181 days of typical weather. The second set contained planned changes in 15 parameters of thermal characteristics and MPR test lengths. The results of these tests are presented in Section 3.

In addition to the comparisons and the sensitivity analysis, an analysis of the measurement error in the estimation of the heat loss coefficient and heating system efficiency is presented in Appendix A. The analysis follows the approach in the ANSI/ASME Standard on Uncertainty Analysis and is based on the presentations of Abernathy et al. (1985) and Coleman and Steele (1989). The results suggest that the measurement uncertainty in the UA estimate ranges between 1 and 5 percent of typical UA values. The measurement of uncertainty in the heating system efficiency estimate is about 1.5 percent for a MPR heating system efficiency estimate of 50 percent. 


\section{Section 2}

\section{MEASURED FUEL CONSUMPTION COMPARED TO MPR PREDICTIONS}

The first approach in the uncertainty analysis was to collect and compare measured fuel consumption data to MPR predictions of fuel use for four previously tested homes. The measured fuel consumption data were collected by an elapsed time meter in one home (Winchester home) and through the local utility company for the other three homes.

The elapsed time meter installed on the Winchester home furnace captured the cumulative on-time of the gas valve. Readings were taken once a week of the furnace elapsed time meter, the gas meter, the electric meter, and a thermometer in the thermostat located in the home.

Utility bill data were collected for the three homes located in New York State. The data were analyzed using a statistical tool, the Princeton Scorekeeping Method (PRISM)(Fels 1986). Two of these homes had participated in weatherization tests. Repeated tests were performed in an occupied third home (the Tully home). The results of these tests were recently reported by the Research Center (1991).

The predictions of fuel consumption for each home were made by the Research Center and are shown in Appendix B. Estimates of monthly fuel consumption were made using the variable base degree-day method. Internal gains were estimated from field audits for the three New York State homes. The internal gains for the Winchester home were estimated from the sum of electricity consumption and 10 percent (SERI 1984) of the gas consumption not used by the furnace. Solar gains were estimated using textbook methods such as presented by Duffie and Beckman (1980) and outlined in Appendix C. The following section summarizes the utility bill analysis of two weatherized homes; details are also presented in Appendix C. 


\section{RESULTS OF TWO WEATHERIZED HOMES}

The heating system in weatherization house \#1 is gas-fired and forced-air. The gas consumption data for house \#1 were well described by the PRISM model. Both pre- and post-weatherization gas consumption data exhibited a linear relationship with outdoor temperature. For the pre-weatherization period, the MPR predictions were well correlated with the utility bill data. Two of the MPR data points were within one standard error of the estimate based on the linear regression, while the third point was very close to one standard error. Four of the utility bill data points fell outside one standard error, but were within two standard errors. The reference temperature (the outdoor temperature at which heating is first required) predicted by PRISM was found to be $65^{\circ} \mathrm{F}\left( \pm 3.7^{\circ} \mathrm{F}\right)$. The MPR method predicted an average reference temperature of $62.6^{\circ} \mathrm{F}$.

The two MPR predictions of monthly fuel consumption in house \#1 for the postweatherization period were within two standard errors of the regressed line fit to the utility bill data. The data from three of the 10 utility bills also fell within two standard errors of the linear regression. The PRISM reference temperature for the postweatherization period was found to be $65.3^{\circ} \mathrm{F}\left( \pm 2.1^{\circ} \mathrm{F}\right)$, which is not significantly different from the pre-weatherization period. The MPR method predicted an average reference temperature of $60.2^{\circ} \mathrm{F}$ for the two months analyzed.

Weatherization house $\# 2$ is heated by an oil-fired furnace. The billing data did not fit a linear trend. In both pre- and post-periods there is a wide scatter in the billing data, and in both cases the reference temperature was well above $80^{\circ} \mathrm{F}$. The MPR method predicted

a reference temperature of $69^{\circ} \mathrm{F}$ for the pre-period and $63^{\circ} \mathrm{F}$ for the post-period. Two of the four MPR predictions fall within the general range of the raw billing data in the preweatherization case. The three MPR predictions fall within the general range of the raw billing data in the post-weatherization case. No attempt was made to compare the MPR predictions with the standard error of estimation because the apparent reference temperature was so high, which indicates a potential problem in trying to apply PRISM analysis (or even making assumptions about the linearity of the data). 
Occupant activity can dramatically influence fuel usage, which may have been the reason for the non-linear energy usage of the house. Many factors could have caused the aberrant energy performance. Sporadic events such as doors and windows opening, erratic thermostat settings, and partially filled fuel tank and frequency of fuel billing could contribute to the disorderly behavior. Consequently, no inferences should be made from these data as to the suitability of the MPR method to oil-heated houses. Rather it points to the need of ensuring that pre- and post-weatherization assessments be conducted under similar occupant circumstances. Unoccupied status for both periods (pre and post) would be optimum and the least desirable comparison would be during dissimilar occupancy.

\section{RESULTS OF TWO PREVIOUSLY TESTED OCCUPIED HOMES}

The Tully house is heated by a gas furnace. The house's gas furnace heating consumption was not well described by a linear trend. The average number of days in the billing periods was 61.3 (range: 59-63). In order to bring the periods of MPR's predictions into agreement with the billing data periods, MPR estimates were combined for the months of October and November, for the months of December and January and for the months of February and March.

Two of MPR's predictions fell within the general range of the raw billing data; one of the MPR values overestimates consumption. MPR predicted a reference temperature of $50^{\circ} \mathrm{F}$, which is based on the audit data but is not matched by the distribution of billing data in that range of average outdoor temperatures. For the period of October to February, MPR predicted a fuel consumption over twice as indicated by the billing data. However, it appears that PRISM predicts a reference temperature well over $90^{\circ} \mathrm{F}$. Clearly, this house was difficult to model with both PRISM and the MPR system.

The final comparison was on the Winchester house, which is about 100 years old and is heated by a gas forced-air heating system. The "best" MPR prediction of gas consumption over the reference period was $67.8 \mathrm{MMBtu}$ (million Btu). This estimate was based or the average UA and heating system efficiency over five tests. The measured furnace gas consumption was $68 \mathrm{MMBtu}$. Appendix B presents the summary of MPR 
predictions for the five tests. All five estimates are within two standard deviations of the measured consumption. The bias in the predicted consumption was about 4 percent of the measured consumption. The scatter resulted in a precision of 26 percent of the mean predicted UA. Therefore, with the Winchester house, most of the error was random and not systematic. 
Section 3

\section{SENSITIVITY ANALYSIS OF TWO PREVIOUSLY TESTED HOMES}

The sensitivity analysis of two previously tested homes was provided by Duffy and is attached as Appendix D. The simulation models were then developed to characterize these homes (Winchester and Bowie). The models were used to estimate the sensitivity to several key parameters: varying outside temperatures, basement and slab-on-grade ground loss and thermal mass, infiltration, mass in exterior walls, multizone heat transfer, and effective sky temperature.

The models of the Winchester and Bowie homes were calibrated with actual MPR test data. These calibrations adjusted the thermal resistances and conductances until an energy balance was achieved. Numerical problems occurred, particularly with the Bowie model, because of the lack of specialized data, such as cool-down test data. The actual UA values are, therefore, estimated by adjusting the model to fit the MPR data.

Simulations were then run using historical weather data and after changing the input values of key parameters. The output of the simulations are indoor and outdoor temperatures and heater on-times. These are input into the MPR analysis code for prediction of the UA and heating system efficiency.

Two approaches were used in the sensitivity tests. 'The first approach used an entire heating season of hourly weather data (TMY) for the respective locations of each home. The simulations and MPR analyses were repeated each night for 181 days of typical weather. The second approach consisted of planned changes of 15 parameters of thermal characteristics and lengths of MPR tests.

\section{RESULTS OF HEATING SEASON SIMULATIONS}

The heating season simulations on the Winchester home resulted in a mean predicted UA that was 10 percent less than the mean actual UA. The random component (standard deviation) was found to be approximately 3 percent of the mean. Furnace efficiency was 
overpredicted by 10 percent with a random component of 10 percent. Two parameters that were found to significantly affect the UA estimates were outdoor temperature and infiltration.

The simulations suggested that the indoor temperature should be about $20^{\circ} \mathrm{F}$ to $30^{\circ} \mathrm{F}$ higher than ambient. This range appears to offer the best tradeoff between bias and random errors. The infiltration component of the UA was the most variable component of those modeled. The significance of the parameter verified the importance of obtaining infiltration data, preferably by tracer gas, but at least by blower door measurements and models. Additional regressions of the effect of solar gains and drifting outdoor temperature indicated no significant relationships.

The heating season simulations indicated little effect of heating system efficiency estimates to outdoor temperatures and to differences between predicted and actual UA.

The Bowie model was less well-defined than the Winchester model. As a result, there was more scatter between predicted UA and actual UA. The mean predicted UA was about 35 percent less than the actual UA. The predicted heating system efficiency was about 30 percent lower than actual efficiency. One of the reasons for the increased bias and scatter in the Bowie home is the forced-air distribution located in the slab. The simulations and multi-parameter analysis suggested that the UA of a slab-on-grade (and a basement) should be estimated separately from the above-grade UA. Some success has been achieved (Gouveia 1993) in extending the MPR method to estimate the UA components for slab-on-grade houses.

\section{RESULTS OF MULTI-PARAMETER ANALYSIS}

The multi-parameter analysis was concerned with the effects of parameters other than weather: thermal mass, heating system distribution patterns, and the interactions of several parameters. Duffy used a matrix approach to screen for critical parameter effects. This greatly reduced the computer time of a typical two-level experimental design which could require over 30,000 simulations for 15 parameters. The matrix approach also is 
based on two levels: a default level based on the heating season analysis and a level that represented a change by a factor of two (in most cases). Details again may be found in Appendix D.

For the Winchester home, reducing wall exterior mass had the largest impact on the predicted UA. Reducing infiltration loss, reducing furnace air to the heated zone, and increasing interzone heat transfer aiso appear to stand out as having large impacts.

For the Bowie house, reducing the furnace output to the air while increasing the output to the slab by a factor of two had a dramatic effect on both the UA and heating system efficiency predictions. Duffy reports:

In fact, in at least one (simulated) test involving a decreased initial slab temperature along with the increased furnace output to the slab, the furnace was on all day and increased the slab heat content enough so that the furnace and heaters essentially did not go on the entire night. Consequently, the estimated UA was zero. Obviously, the implication for houses with in-slab heating is to monitor the initial slab conditions prior to MPR testing. 


\section{Section 4}

\section{IMPLICATIONS OF THE UNCERTAINTY ANALYSIS RESULTS}

The following approaches were used in the uncertainty analysis of the MPR system: 1) comparing MPR predicted fuel consumption to the utility bill data for three homes and to the measured furnace consumption for one home; and 2) a sensitivity analysis using simulated MPR tests with historical weather data and varying thermal characteristics. Both approaches were useful in estimating the probable effects of bias and scatter on UA and heating system efficiency estimates.

A third approach looked at the measurement bias and scatter that is propagated into the UA and heating system efficiency estimates. These error sources are inherent to some degree in all measurement applications. The results presented in Appendix A suggest that the uncertainty due to measurement error may range from 1 percent to 5 percent of typical UA values, and about 3 percent of a heating system efficiency estimate of 50 percent.

The heating season simulations of the Winchester home indicated bias and random errors both about 15 percent of the mean UA. In comparison, measurement error would be about 2.5 percent of the mean UA. The bias of the heating system efficiency estimate was 18 percent and the scatter was about 9 percent of the mean.

The results of the Bowie house indicated much more uncertainty. In addition to real sensitivities, some of the uncertainty is also believed to be due largely to the model rather than the MPR method.

The comparisons between utility bill data and actual consumption indicated problems with both the MPR approach as well as PRISM's approach. Two of the homes' consumption were well defined by the measured data: weatherization house $\# 1$ and the Winchester home. The MPR predictions for those two homes were in most cases within one standard deviation of the measured data. In fact, for the Winchester house, a comparison of 
predicted fuel consumption based on MPR tests to measured fuel consumption yielded ar average difference of only 0.3 percent and a standard deviation of 4 percent. However, the other two homes were more difficult to analyze with PRISM. Indeed, both predicted unrealistic reference temperatures that differed considerably from the audit information.

The results of both approaches suggest that the bias and random errors in the MPR system vary from house to house and over different weather conditions. In some cases the MPR system appears to characterize the house well. But in others, MPR and PRISM fail to model energy consumption adequately. This suggests that for some complicated structures, such as ground-coupled heat loss, the basic MPR protocol does not adequately characterize the heating requirements.

MPR protocols were not designed to examine large thermal mass effects such as widely variable internal gains, heating ducts in or below slab foundations, and passive solar buildings with thermal mass storage. However, Duffy estimates that MPR can detect UA changes as low as 7 percent tused on single before and after tests. Also, MPR estimates of UA compare well with other similar analytical tools (Research Center 1992) in light frame and unoccupied houses.

Duffy questions the wisdom of using a single UA to represent the thermal efficiency of a building that is strongly ground-coupled, like the slab-on-grade Bowie house. There should be two heat loss coefficients examined separately: the UA from the heated zone to the ambient air and the UA from the heated zone to the earth. This would require modifying the test protocol to include extra tests. Such modifications could add one to two extra days of testing but would appear to greatly reduce the uncertainty of the MPR system.

Based on the results presented herein, the following observations were made by Duffy and are concurred by the Research Center: It appears that the MPR method is reasonably precise for large field applications (for which it was originally intended). For weatherization studies, with a single before and a single after test, a change of 7 percent 
in UA should be detectable at the 95 percent confidence level. The bias in UA predictions and associated energy estimates can be reduced by applying regression models developed by Duffy for sensitivity to ambient temperatures and infiltration levels. Additional bias correction equations could be developed with further simulations and more complete experimental designs focused on the most sensitive parameters. Initial conditions at the time of the start of the tests must be monitored and accounted for in the analysis. Further development of non-steady-state models and ramp-up-type corrections need to be undertaken. Differences between the indoor and outdoor temperatures of at least $20^{\circ} \mathrm{F}$ should be maintained by raising the indoor thermostat set points if necessary.

Synertech's final report recommends re-examining the principles of MPR before applying it to weatherization programs. This comment is especially relevant if the objective is to replicate utility bills. Reconciling differences between an estimate and utility bill is flawed and a somewhat meaningless approach because of the potential impact occupants have on energy consumption. All energy estimation models require assumptions of occupant behavior; the basic assumption being that occupants are predictable and uniform. However, occupants may not follow the model's estimate of behavior; i.e. doors or windows may be left open, thermostat settings may be changed frequently, etc. These events would account for differences between estimated and actual utility bills. The difficulties encountered in this study in using fuel bill data in estimating changes in UA point even more to the need of an MPR method to detect UA improvements due to weatherization.

At this time, the overall best method of uncertainty prediction in the field appears to be the system identification method (Bourque and Duffy 1992) of estimating the model equation error in real time given the measurement error estimates (which are relatively more easily predicted).

In this study, it was obvious that most of the difficulties and uncertainties were due more to the methods of estimating the true UA, furnace efficiency, and furnace fuel consumption (by collecting fuel bills and simulating two actual houses) than to the MPR 
approach. As some evidence for the statement for the Winchester house for which the true parameters were known with more certainty, the MPR predictions compared more favorably. Consequently, in retrospect, these uncertainties become arguments in favor of the need of an empirical measure of UA and furnace efficiency, like the MPR method, rather than arguments against. In other words, it appears that simulations and estimates of UA and furnace efficiency based on fuel bills have more uncertainty than the MPR method. 


\section{Section 5}

\section{RECOMMENDATIONS FOR FUTURE WORK}

At this time, the overall best method of uncertainty prediction in this field appears to be the system identification method (Bourque and Duffy 1992) of estimating the model equation error in real time given the measurement error estimates (which are relatively more easily predicted). The MPR method should be extended to predict both the UA-toair and the UA-to-earth, separately using the method of Gouveia (1993). A somewhat simplified version of the analytic approach of Bourque and Duffy (1992) with a series of coheat, furnace, and cooldown tests would probably be adequate to predict both UA components. Of course, more study would be needed here. In addition, the test protocols would need to be modified to incorporate the changes in the analytic approach.

Given the extent of investment into the thermal network simulation models, we also recommend that further simulation be performed on the parameters outdoor temperature and infiltration that appear to largely impact the MPR results. Such simulation should provide insight into the best approach for revising the test protocol.

One other result of this analysis is worth noting. The MPR predictions of furnace efficiencies for the Winchester house model overestimated the actual by 10 percent on average. Many of the MPR predictions of furnace efficiencies obtained in the field were low, with some even in the range of 30 percent (Research Center 1991). If, in fact, the actual heating system efficiencies were even lower, the implications for the need of weatherization and upgrades of heating systems and for the need of a method such as the MPR to test entire heating systems is clear. 


\section{REFERENCES}

1. Abernathy, R.B., R.P. Benedict, and R.B. Dowdell. "ASME Measurement Uncertainty," ASME Journal of Fluids Engineering, Vol. 107. 1985.

2. ASHRAE. Handbook of Fundamentals. American Society of Heating, Refrigerating and Air-Conditioning Engineers, Atlanta, GA. 1989.

3. Bourque, K., and J.J. Duffy. "Building Thermal Parameter Estimation using System Identification and Short-Term Tests," Proceedings of the 1992 National Solar Energy Conference. American Solar Energy Society, Boulder, CO. 1992.

4. Coleman, H.W., and W.G. Steelc. Experimentation and Uncertainty Analysis for Engineers. John Wiley \& Sons, New York. 1989.

5. Duffie, J.A., and W.A. Beckman. Solar Engineering of Thermal Processes. John Wiley \& Sons, New York. 1980.

6. Duffy, J.J., and H. Puri. "Extrapolation of Short-Term Measurements to LongTerm Thermal Performance of Passive Buildings," Proceedings of the 10th National Passive Solar Conference. American Solar Energy Society, Boulder, CO. 1985.

7. Duffy, J.J., and D.H. Saunders. 'Low-cost Methods for Evaluation of Space Conditioning Efficiency of Existing Homes," University of Lowell final report to NAHB Research Center. 1987.

8. Duffy, J.J., D.H. Saunders, and J. Spears. "Low-cost Methods for Evaluation of Space Conditioning Efficiency of Existing Homes," Proceedings of the 1987 Passive Solar Conference, American Solar Energy Society, Boulder, CO. 1987.

9. Fels, M.F. ed. "Measuring Energy Savings: The Scorekeeping Approach," Energy and Buildings. Vol. 9, No. 1 \& 2. 1986.

10. Gouveia, Brett. "Sensitivity Analysis of the Measured Performance Rating Method." MS Thesis, University of Massachusetts, Lowell. 1993.

11. Research Center. Short-Term Test Methods for Predicting the Thermal I'erformance of Buildings, NAHB Research Center final report to the New York State Energy Research and Development Authority, 1129-EEED-AEP-89. 1991.

12. Research Center. Comparison of Two Building Thermal Test Methods, EPRI TR101478. Palo Alto, CA, 1992.

13. SERI. Passive Solar Performance, Summary Class B Results, Solar Energy Research Institute Report, SERI/SP-271-2362, Golden, CO. 1984. 
14. Sherman, M., and D. Grimsrud. Measurement of infiltration Using Fan Pressurization and Weather Data. Lawrence Berkeley Laboratory report LBL10852. Berkeley, CA, 1982. 


\section{APPENDIX A}




\section{HEAT LOSS COEFFICIENT ESTIMATE}

The data reduction equation for the heat loss coefficient (UA) $\left(\mathrm{Btu} / \mathrm{hr}-{ }^{\circ} \mathrm{F}\right)$ is:

$$
\mathrm{UA}=\frac{\mathrm{Q}}{\Delta \mathrm{T}}=\frac{\sum_{\mathrm{i}=0}^{60} \mathrm{C} \cdot \mathrm{V} \cdot \mathrm{I}}{\Delta \mathrm{T}}
$$

where $Q$ is the heating energy provided to the building by the electric space heaters during the coheating test and $\Delta \mathrm{T}$ is the measured indoor-outdoor temperature difference $\left({ }^{\circ} \mathrm{F}\right)$. $\mathrm{Q}$ is the product of two measured parameters, voltage (V) and electric current (I) in amperes. The hourly equivalent of UA is evaluated every fifteen minutes based on fifteen second scan frequency; thus every estimate of UA is evaluated from 60 observations of energy consumption and temperature differential. The constant $(\mathrm{C})$ provides conversion to appropriate energy and time units.

Abernathy et al. (1985) present two methods of combining the bias and precision errors. The first method provides a 99 percent confidence level and is combination by straight addition. The second method provides 95 percent coverage and is combination by root-sum-square (RSS). The analysis presented here follows the RSS method

$$
U_{r}=\left[B^{2}+\left(P_{r}\right)^{2}\right]^{1 / 2}
$$

where $U_{r}$ is the uncertainty of a result, $B$ is the bias limit and $P_{r}$ is the precision index of the result. The precision index is estimated by

$$
P_{r}=t \cdot S_{r}
$$

where $S_{r}$ is the standard deviation of the result. The Student $t$ value is a function of the degrees of freedom used in calculating $S_{r}$.

\section{Bias Limit Estimation}

To determine the overall bias and precision limits of the UA estimate we need to estimate the bias limits and precision limits of the variables in the UA estimate. First, the bias limits for the measurement devices currently being used are estimated. The estimates are based on the manufacturer's specifications, and in part on the judgement of those who have used the instruments in experimental applications. The estimated bias limits for the temperature, voltage and current measurements are listed in Table A-1. 
Table A-1

Bias limits for variables in the UA data reduction equation.

\begin{tabular}{||l|c|}
\hline \multicolumn{1}{|c|}{ Variable } & Bias Limit \\
\hline \hline Indoor temperature $\left(T_{i}\right)$ & $\pm 1.0^{\circ} \mathrm{F}$ \\
\hline Outdoor temperature $\left(T_{0}\right)$ & $\pm 1.0^{\circ} \mathrm{F}$ \\
\hline Voltage $(V)$ & \pm 1.0 Volts \\
\hline Current $1\left(i_{1}\right)$ & $\pm 0.6 \mathrm{~A}$ \\
\hline Current $2\left(i_{2}\right)$ & $\pm 0.6 \mathrm{~A}$ \\
\hline
\end{tabular}

Following Abernathy et al. (1985), the uncertainty analysis equation for bias is

$$
B_{U A}^{2}=\left(\frac{\partial U A}{\partial V}\right)^{2} B_{V}^{2}+\left(\frac{\partial U A}{\partial I}\right)^{2} B_{I}^{2}+\left(\frac{\partial U A}{\partial \Delta T}\right)^{2} B_{\Delta T}^{2}
$$

The bias due to $\Delta T$ and $I\left(i_{1}+i_{2}\right)$ are nonindependent: the temperature sensors were calibrated at the same time against the same reference temperature sensor, and placed adjacent to each other at essentially the same location; and the current sensors were calibrated at the same time against the same factory-calibrated reference current transducer. The procedure for estimating nonindependent bias limits is outlined in Coleman and Steele (1989). In this case, the bias limits of the indoor and outdoor temperature measurements are correlated, and the current measurements of the two 120 Volt service lines are correlated. The uncertainty expression for propagation of the bias limits of the temperature measurements into the UA estimate is

$$
\mathrm{B}_{\Delta \mathrm{T}}^{2}=\left(\frac{\partial \Delta \mathrm{T}}{\partial \mathrm{T}_{\mathrm{i}}}\right)^{2} \mathrm{~B}_{\mathrm{T}_{\mathrm{i}}}^{2}+\left(\frac{\partial \Delta \mathrm{T}}{\partial \mathrm{T}_{0}}\right)^{2} \mathrm{~B}_{\mathrm{T}_{0}}^{2}+2\left(\frac{\partial \Delta \mathrm{T}}{\partial \mathrm{T}_{\mathrm{i}}}\right)\left(\frac{\partial \Delta \mathrm{T}}{\partial \mathrm{T}_{0}}\right) \rho_{\mathrm{T}_{\mathrm{T}} \mathrm{T}_{0}} \mathrm{~B}_{\mathrm{T}_{1}} \mathrm{~B}_{\mathrm{T}_{0}}
$$

We have assumed that there is perfect correlation so that the coefficient of correlation ( $\rho)$ is set to unity. Substituting the bias limits of the temperature measurements and the partial derivatives into Equation 5 results in an overall bias limit for the temperature difference of zero:

$$
\mathrm{B}_{\Delta \mathrm{T}}^{2}=(1)^{2}(1)^{2}+(-1)^{2}(1)^{2}+2(1)(-1)(1)(1)(1)=0
$$

Similarly, the bias limit for the nonindependent current measurements is found to equal $1.2 \mathrm{~A}$. 
The partial derivatives of the data reduction equation are

$$
\frac{\partial U A}{\partial V}=\frac{I}{\Delta T}, \frac{\partial U A}{\partial I}=\frac{V}{\Delta T}, \frac{\partial U A}{\partial \Delta T}=\frac{-V \cdot I}{\Delta T^{2}}
$$

Since the bias limit due to the temperature difference was found to equal zero, Equation 4 reduces to

$$
\mathrm{B}_{\mathrm{UA}}^{2}=\left(\frac{\mathrm{I}}{\Delta \mathrm{T}}\right)^{2} \mathrm{~B}_{\mathrm{V}}^{2}+\left(\frac{\mathrm{V}}{\Delta \mathrm{T}}\right)^{2} \mathrm{~B}_{1}^{2}
$$

If we assume nominal values fcr the parameters that are equivalent to typical values during a coheat test -- 240 Volts, 40 amp hours (about 80 percent heater on-time) and $30^{\circ} \mathrm{F} \mathrm{--}$ then the square of the bias limit for the UA estimate becomes

$$
B_{U A}^{2}=\left(\frac{40}{30}\right)^{2}(1)^{2}+\left(\frac{240}{30}\right)^{2}(1.2)^{2}=94
$$

\section{Precision Limit Estimation}

The uncertainty equation for the precision limit is similar to that for the bias limit

$$
\mathrm{P}_{U A}^{2}=\left(\frac{\partial U A}{\partial V}\right)^{2} P_{V}^{2}+\left(\frac{\partial U A}{\partial I}\right)^{2} P_{I}^{2}+\left(\frac{\partial U A}{\partial \Delta T}\right)^{2} P^{2}{ }_{\Delta T}
$$

The precision limits were estimated at the 95 percent confidence level using Equation 3 and repeated measurements at fixed conditions. Table A-2 lists the estimated precision limits for the temperature, voltage and current measurements. 
Table A-2

Precision limits for variables in the UA data reduction equation.

\begin{tabular}{||l|c|}
\hline \multicolumn{1}{|c|}{ Variable } & Precision Limit \\
\hline \hline Indoor temperature $\left(\mathrm{T}_{\mathrm{i}}\right)$ & $\pm 0.4^{\circ} \mathrm{F}$ \\
\hline Outdoor temperature $\left(\mathrm{T}_{0}\right)$ & $\pm 0.4^{\circ} \mathrm{F}$ \\
\hline Voltage $(\mathrm{V})$ & \pm 0.6 Volts \\
\hline Current 1 $\left(\mathrm{i}_{1}\right)$ & $\pm 0.2 \mathrm{~A}$ \\
\hline Current 2 $\left(\mathrm{i}_{2}\right)$ & $\pm 0.2 \mathrm{~A}$ \\
\hline
\end{tabular}

When the temperature precision limits are combined by RSS method, the resulting limit is $\pm 0.6^{\circ} \mathrm{F}$. Similarly, using the RSS method to combine the precision limits of the current measurements results in a limit of $\pm 0.28 \mathrm{~A}$. Substituting the partial derivatives and precision limits into Equation 10 results in

$$
P_{U A}^{2}=\left(\frac{40}{30}\right)^{2}(0.6)^{2}+\left(\frac{240}{30}\right)^{2}(0.28)^{2}+\left(\frac{240 \cdot 40}{30^{2}}\right)^{2}(0.6)^{2}=47
$$

The measurement uncertainty of the UA estimate is found by substituting the results of Equations 9 and 11 into Equation 2

$$
\mathrm{U}_{\mathrm{UA}}=(94+47)^{1 / 2}= \pm 12 \mathrm{Btu} / \mathrm{hr}{ }^{\circ} \mathrm{F}
$$

The result in Equation 12 is about 1 percent of a UA of $1090 \mathrm{Btu} / \mathrm{h}^{\circ} \mathrm{F}$ estimated from the nominal values. That $U A$ represents the high end of the range of UA estimates. The uncertainty is about 5 percent of $200 \mathrm{Btu} / \mathrm{hr}^{\circ} \mathrm{F}$ which may be considered as the low end of the range.

\section{HEATING SYSTEM EFFICIENCY ESTIMATE}

The data reduction equation for the overall heating system efficiency estimate is

$$
\eta=\frac{(U A \cdot \Delta T-E)}{W \cdot t_{\text {on }}}
$$


Bias Limit Estimation

Table A-3

Bias and precision limits for variables in the heating system efficiency data reduction equation.

\begin{tabular}{|l|c|c|}
\hline \multicolumn{1}{|c|}{ Variable } & Bias Limit & Precision Limit \\
\hline \hline Indoor temperature $\left(\mathrm{T}_{1}\right)$ & $\pm 1^{\circ} \mathrm{F}$ & $\pm 0.4^{\circ} \mathrm{F}$ \\
\hline Outdoor temperature $\left(\mathrm{T}_{0}\right)$ & $\pm 1^{\circ} \mathrm{F}$ & $\pm 0.4^{\circ} \mathrm{F}$ \\
\hline Heat loss coefficient $(\mathrm{UA})$ & $\gamma^{\prime} .7 \mathrm{Btu} / \mathrm{hr}{ }^{\circ} \mathrm{F}$ & $\pm 6.9 \mathrm{Bru} / \mathrm{hr}^{\circ} \mathrm{F}$ \\
\hline Internal gains $(\mathrm{E})$ & $\pm 115 \mathrm{Btu}$ & $\pm 132 \mathrm{Btu}$ \\
\hline Fuel input rate $(\mathrm{W})$ & $\pm 1000 \mathrm{Btuh}$ & $\pm 3000 \mathrm{Btuh}$ \\
\hline System on-time $\left(\mathrm{t}_{\mathrm{on}}\right)$ & 0 & 0 \\
\hline
\end{tabular}

The bias and precision limits for the variables in Equation 13 are listed in Table A-3. We have assumed that the bias and precision limits for "time" and the "on-time" of the furnace are zero. The uncertainty equation for the bias limit of the heating system efficiency is

$$
\begin{aligned}
\mathrm{B}_{\eta}^{2}=\left(\frac{\partial \eta}{\partial \mathrm{T}_{\mathrm{i}}} \mathrm{B}_{\mathrm{T}_{1}}\right)^{2} & +\left(\frac{\partial \eta}{\partial \mathrm{T}_{\mathrm{o}}} \mathrm{B}_{\mathrm{T}_{0}}\right)^{2}+\left(\frac{\partial \eta}{\partial \mathrm{UA}} \mathrm{B}_{\mathrm{UA}}\right)^{2}+\left(\frac{\partial \eta}{\partial \mathrm{E}} \mathrm{B}_{\mathrm{E}}\right)^{2} \\
& +\left(\frac{\partial \eta}{\partial \mathrm{W}} \mathrm{B}_{\mathrm{w}}\right)^{2}+2 \frac{\partial \eta}{\partial \mathrm{T}_{\mathrm{i}}} \frac{\partial \eta}{\partial \mathrm{T}_{0}}(1) \mathrm{B}_{\mathrm{T}}^{2}
\end{aligned}
$$

The partial derivatives are

$$
\begin{aligned}
& \frac{\partial \eta}{\partial T_{i}}=\frac{U A}{W \cdot t_{o n}} \\
& \frac{\partial \eta}{\partial T_{o}}=-\frac{U A}{W t_{o n}} \\
& \frac{\partial \eta}{\partial U A}=\frac{\Delta T}{W \cdot t_{o n}}
\end{aligned}
$$




$$
\begin{gathered}
\frac{\partial \eta}{\partial E}=-\frac{1}{W \cdot t_{\text {on }}} \\
\frac{\partial \eta}{\partial W}=-\frac{(U A \cdot \Delta T-E)}{W^{2} \cdot t_{\text {on }}}=-\frac{\eta}{W} \\
\frac{\partial \eta}{\partial t_{\text {on }}}=-\frac{(U A \cdot \Delta T-E)}{W \cdot t^{2}{ }_{\text {on }}}=-\frac{\eta}{t_{\text {on }}}
\end{gathered}
$$

In Equation 14, the terms containing the temperature bias limit add to zero, and the term containing the bias limit for the system on-time is zero. Substituting the remaining partial derivatives into Equation 14 results in

$$
B_{\eta}^{2}=\left(\frac{T_{i}-T_{o}}{W \cdot t_{o n}}\right)^{2} B_{U A}^{2}+\left(\frac{1}{W \cdot t_{o n}}\right)^{2} B_{E}^{2}+\left(\frac{\eta}{W}\right)^{2} B^{2}{ }_{w}
$$

Nominal values for the parameters in the heating system efficiency data reduction equation are listed in Table A-4, and are based on a four hour heating system efficiency test.

Table A.4

Nominal values for variables in the heating system efficiency data reduction equation.

\begin{tabular}{||l|c||}
\hline \multicolumn{1}{|c|}{ Variable } & Value \\
\hline \hline Indoor temperature $\left(T_{i}\right)$ & $70^{\circ} \mathrm{F}$ \\
\hline Outdoor temperature $\left(T_{0}\right)$ & $40^{\circ} \mathrm{F}$ \\
\hline Heat loss coefficient $(U A)$ & $400 \mathrm{Btu} / \mathrm{hr}^{\circ} \mathrm{F}$ \\
\hline Internal gains $(\mathrm{E})$ & $4,800 \mathrm{Btu}$ \\
\hline Fuel input rate $(\mathrm{W})$ & $100,000 \mathrm{Btuh}$ \\
\hline System on-time $\left(\mathrm{t}_{\mathrm{on}}\right)$ & $1 \mathrm{hr}$ \\
\hline Efficiency & $50 \%$ \\
\hline
\end{tabular}


Substituting the bias limits from Table A-3 and the nominal values from Table A-4 into Equation 21 results in

$$
\begin{aligned}
B_{\eta}^{2}=\left(\frac{30}{100000 \cdot 1}\right)^{2}(9.7)^{2} & +\left(\frac{1}{100000 \cdot 1}\right)^{2}(115)^{2}+\left(\frac{0.5}{100000}\right)^{2}(1000)^{2} \\
& =0.348\left(10^{-6}\right)
\end{aligned}
$$

The square root of the result is 0.006 or 0.6 percent.

\section{Precision Limit Estimation}

The precision limit is found using

$$
\mathrm{P}_{\eta}^{2}=\left(\frac{\partial \eta}{\partial \mathrm{T}_{\mathrm{i}}} \mathrm{P}_{\mathrm{T}_{i}}\right)^{2}+\left(\frac{\partial \eta}{\partial \mathrm{T}_{\mathrm{o}}} \mathrm{P}_{\mathrm{T}_{0}}\right)^{2}+\left(\frac{\partial \eta}{\partial \mathrm{UA}} \mathrm{P}_{\mathrm{UA}}\right)^{2}+\left(\frac{\partial \eta}{\partial \mathrm{E}} \mathrm{P}_{\mathrm{E}}\right)^{2}+\left(\frac{\partial \eta}{\partial \mathrm{W}} \mathrm{P}_{\mathrm{W}}\right)^{2}+\left(\frac{\partial \eta}{\partial \mathrm{t}_{0 n}} \mathrm{P}_{\mathrm{I}_{-}}\right)^{2}
$$

Since we have assumed that the precision limit of the system on-time measurement is zero (this has not, however, been field verified), the term containing that limit equals zero. Substituting the partial derivatives into Equation 23 results in

$$
P_{\eta}^{2}=\left(\frac{U A}{W \cdot t_{o n}}\right)^{2} P_{T_{i}}^{2}+\left(\frac{U A}{W \cdot t_{o n}}\right)^{2} P^{2} T_{0}+\left(\frac{\Delta T}{W \cdot t_{o n}}\right)^{2} P_{E}^{2}+\left(\frac{\eta}{W}\right)^{2} P^{2}{ }_{W}
$$

Substituting the nominal values and precision limits into Equation 24 results in

$$
\begin{aligned}
P_{\eta}^{2}=\left(\frac{400}{100000}\right)^{2}(0.4)^{2} & +\left(\frac{400}{100000}\right)^{2}(0.4)^{2}+\left(\frac{30}{100000}\right)^{2}(6.9)^{2} \\
& +\left(\frac{1}{100000}\right)^{2}(132)^{2}+\left(\frac{0.5}{100000}\right)^{2}(3000)^{2}=2.36\left(10^{-4}\right)
\end{aligned}
$$

It is evident from Equation 25 that the precision of the fuel input rate; measurement is important when trying to increase the precision of the heating system efficier.iy estimate. The square root of the Equation 25 result equals 0.15 or 1.5 percent. 
The uncertainty (with 95 percent coverage) in the heating system efficiency estimate is found by using Equation 2

$$
U_{\eta}=\left[B_{\eta}^{2}+P_{\eta}^{2}\right]^{1 / 2}
$$

Using the bias and precision limits previously calculated results in

$$
U_{\eta}=\left[(0.6)^{2}+(1.5)^{2}\right]^{1 / 2}= \pm 1.6 \%
$$

Therefore, the measurement uncertainty of the heat loss coefficient estimate was found to be approximately 1 percent to 5 percent of estimates ranging from $200 \mathrm{Btu} / \mathrm{hr}{ }^{\circ} \mathrm{F}$ to $1000 \mathrm{Btu} / \mathrm{hr}{ }^{\circ} \mathrm{F}$, and the measurement uncertainty of the heating system efficiency estimate was found to be about 1.5 percent or 3 percent of a nominal efficiency estimate of 50 percent. The measurement of the furnace fuel rate appears to largely influence the precision of the heating system efficiency estimate. Bias errors of measurement sensors appear to influence the heat loss coefficient estimate more than the precision of the sensors. 
APPENDIX B

SUMMARY OF PREDICTED FUEL USAGE 
Weatherization House \#1

Average indoor temperature $67.8^{\circ} \mathrm{F}$

Solar area $3 \mathrm{ft}^{2}$

Internal gains $23,891 \mathrm{Btu} / \mathrm{day}$

Table B.1

Pre- and Post-Predictions for Weatherization

House \#1

\begin{tabular}{|c|c|c|c|c|c|c|c|c|c|}
\hline \multicolumn{10}{|c|}{$\begin{array}{l}\text { Pre-period October } 1,1990 \text { to December } 31,1990 \\
\text { UA }-201 \text { Btu/hro } \\
\text { Heating system efficiency }=0.36\end{array}$} \\
\hline Month & Days & $\begin{array}{l}\text { Outdoor } \\
\text { Temperanure } \\
{ }^{\circ} \mathrm{F}\end{array}$ & $\begin{array}{l}\text { Degree } \\
\text { Days }\end{array}$ & $\begin{array}{c}\text { Total Load } \\
\text { Bru }\end{array}$ & $\begin{array}{l}\text { Solar } \\
\text { Gains } \\
\text { Btu }\end{array}$ & $\begin{array}{l}\text { Internal } \\
\text { Gains } \\
\text { Bu }\end{array}$ & $\begin{array}{c}\text { Net Load } \\
\text { Bns }\end{array}$ & $\begin{array}{c}\text { Fuel Usage } \\
\text { Btu }\end{array}$ & $\begin{array}{l}\text { Reference } \\
\text { Temp. } \\
\text { of }\end{array}$ \\
\hline Oct & 31 & 53.1 & 456 & $2,198,297$ & 64,949 & 740.621 & $1,392,727$ & $3,868,686$ & 62.4 \\
\hline Nov & 30 & 42.5 & 759 & $3,661,416$ & 37,836 & 716,730 & $2,906,850$ & $8,074,583$ & 62.6 \\
\hline Dec & 31 & 33.8 & 1054 & $5,084,496$ & 24,568 & 740,621 & $4,319,307$ & $11,998,075$ & 62.7 \\
\hline \multicolumn{10}{|c|}{ Total fuel usage $-23.9 \mathrm{MMBu}$} \\
\hline \multicolumn{10}{|c|}{$\begin{array}{l}\text { Post-period February 1, } 1991 \text { to March 31, } 1991 \\
\text { UA }-141 \text { BtwhroF } \\
\text { Heating system efficiency }-0.36\end{array}$} \\
\hline Month & Days & $\begin{array}{l}\text { Outdoor } \\
\text { Temperature } \\
{ }^{\circ} \mathrm{F}\end{array}$ & $\begin{array}{c}\text { Degree } \\
\text { Days }\end{array}$ & $\begin{array}{c}\text { Total Load } \\
\text { Bnu }\end{array}$ & $\begin{array}{c}\text { Solar } \\
\text { Gains } \\
\text { Btu }\end{array}$ & $\begin{array}{l}\text { Intemal } \\
\text { Gains } \\
\text { Btu }\end{array}$ & $\begin{array}{c}\text { Net Load } \\
\text { Btu }\end{array}$ & $\begin{array}{c}\text { Fuel Usage } \\
\text { Btu }\end{array}$ & $\begin{array}{c}\text { Reference } \\
\text { Temp. } \\
{ }^{\circ} \mathrm{F}\end{array}$ \\
\hline Feb & 28 & 30.0 & 1058 & $3,581,626$ & 44,383 & 668.948 & $2,868,295$ & $7,967,485$ & 60.3 \\
\hline Mar & 31 & 37.9 & 927 & $3,136,630$ & 68,729 & 740,621 & $2.327,280$ & $6,464,666$ & 60.1 \\
\hline
\end{tabular}


Weatherization House \#2

Average indoor temperature $74.0^{\circ} \mathrm{F}$

Solar area $46.9 \mathrm{ft}^{2}$

Internal gains $45,256 \mathrm{Btu} /$ day

Table B.2

Pre- and Post-Predictions for Weatherization

House \#2

\begin{tabular}{|c|c|c|c|c|c|c|c|c|c|}
\hline \multicolumn{10}{|c|}{$\begin{array}{l}\text { Pre-period October } 1,1990 \text { to January } 28,1991 \\
\text { UA }-555 \text { Btu/hro } \\
\text { Heating system efficiency }-0.41\end{array}$} \\
\hline Month & Days & $\begin{array}{l}\text { Outdoor } \\
\text { Temperature } \\
{ }^{\circ} \mathrm{F}\end{array}$ & $\begin{array}{c}\text { Degree } \\
\text { Days }\end{array}$ & $\begin{array}{c}\text { Total Load } \\
\text { Btu }\end{array}$ & $\begin{array}{l}\text { Solar } \\
\text { Gains } \\
\text { Btu }\end{array}$ & $\begin{array}{l}\text { Internal } \\
\text { Gains } \\
\text { Bwu }\end{array}$ & $\begin{array}{c}\text { Net Load } \\
\text { Btu }\end{array}$ & $\begin{array}{c}\text { Fuel Usage } \\
\text { Btu }\end{array}$ & $\begin{array}{l}\text { Reference } \\
\text { Temp. } \\
{ }^{\circ} \mathrm{F}\end{array}$ \\
\hline Oct & 31 & 53.1 & 648 & $8,630,028$ & $1,012,211$ & $1,402,936$ & $6,214,881$ & $15,158,246$ & 68.2 \\
\hline Nov & 30 & 42.5 & 945 & $12,587,400$ & 598,944 & $1,357,680$ & $10,630,776$ & $25,928,722$ & 69.1 \\
\hline Dec & 31 & 33.8 & 1246 & $16,599,384$ & 375,169 & $1,402,936$ & $14,821,279$ & $36,149,461$ & 69.7 \\
\hline Jan & 28 & 24.0 & 1400 & $18,648,000$ & 477,096 & $1,267,168$ & $16,903,736$ & $41,228,624$ & 69.3 \\
\hline \multicolumn{10}{|c|}{ Total fuel usage $118.5 \mathrm{MMBtu}$} \\
\hline \multicolumn{10}{|c|}{$\begin{array}{l}\text { Post-period February } 1,1991 \text { to April } 30,1991 \\
\text { UA - } 289 \text { BtwhroF } \\
\text { Heating system efficiency }=0.38\end{array}$} \\
\hline Montb & Days & $\begin{array}{l}\text { Outdoor } \\
\text { Temperature } \\
{ }^{\circ} \mathrm{F}\end{array}$ & $\begin{array}{l}\text { Degree } \\
\text { Days }\end{array}$ & $\begin{array}{c}\text { Total Load } \\
\text { Bur }\end{array}$ & $\begin{array}{c}\text { Solar } \\
\text { Gains } \\
\text { Btu }\end{array}$ & $\begin{array}{l}\text { Internal } \\
\text { Gains } \\
\text { Bru }\end{array}$ & $\begin{array}{c}\text { Net Load } \\
\text { Bus }\end{array}$ & $\begin{array}{l}\text { Fuel Usage } \\
\text { Btu }\end{array}$ & $\begin{array}{l}\text { Reference } \\
\text { Temp. } \\
{ }^{\circ} \mathrm{F}\end{array}$ \\
\hline Feb & 28 & 30.0 & 1232 & $8,545,152$ & 689,170 & $1,267,168$ & $6,588,814$ & $17,338,984$ & 63.9 \\
\hline Mar & 31 & 37.9 & 1119 & $7,762,077$ & $1,076,399$ & $1,402,936$ & $5,282,743$ & $13,901,954$ & 62.5 \\
\hline Apr & 30 & 51.3 & 681 & $4,723,416$ & $1,237,446$ & $1,357,680$ & $2,128,290$ & $5,600,763$ & 61.5 \\
\hline
\end{tabular}


Tully, NY, House

Average indoor temperature setpoint $56.7^{\circ} \mathrm{F}$

Solar area $58.5 \mathrm{ft}^{2}$

Internal Gains 48,260 Btu/day

Best Estimate -- average of 5 tests

$\mathrm{UA}=476 \mathrm{Btu} / \mathrm{hr}^{\circ} \mathrm{F}$

Heating System Efficiency $=0.44$

Table B.3

Prediction Using Average UA and

Heating System Efficiency Estimate

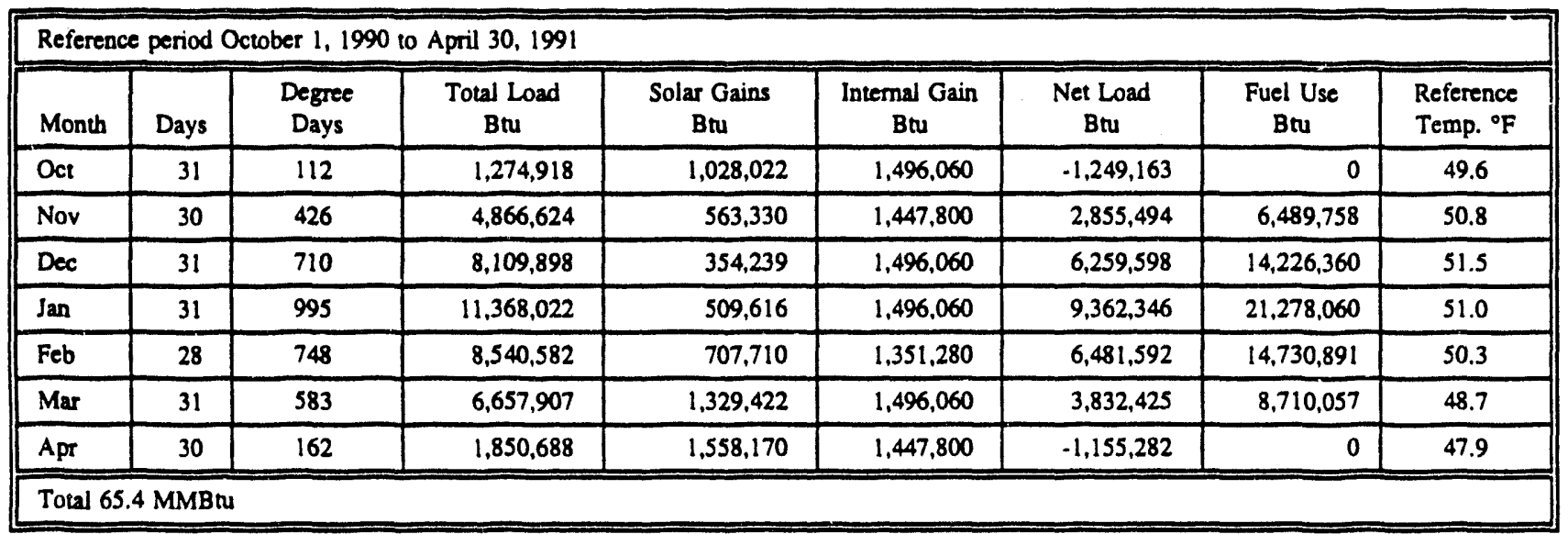

Test 121490

$\mathrm{UA}=477 \mathrm{Btw} / \mathrm{hr}^{\circ} \mathrm{F}$

Heating System Efficiency $=0.48$

Table B.4

Prediction Using Test 121490 Data

\begin{tabular}{|c|c|c|c|c|c|c|c|}
\hline Month & Days & $\begin{array}{l}\text { Degree } \\
\text { Days }\end{array}$ & $\begin{array}{c}\text { Total Load } \\
\text { But }\end{array}$ & $\begin{array}{c}\text { Solar Gains } \\
\text { Btu }\end{array}$ & $\begin{array}{c}\text { Internal Gains } \\
\text { Btu }\end{array}$ & $\begin{array}{l}\text { Net Load } \\
\text { Btu }\end{array}$ & $\begin{array}{c}\text { Fuel Use } \\
\text { Bru }\end{array}$ \\
\hline Oct & 31 & 112 & $1,277,597$ & $1,028,022$ & $1,496,060$ & $-1,246,485$ & 0 \\
\hline Nov & 30 & 426 & $4,876,848$ & 563,330 & $1,447,800$ & $2,865.718$ & $5,970,245$ \\
\hline Dec & 31 & 710 & $8,126,935$ & 354,239 & $1,496,060$ & $6,276,636$ & $13,076,325$ \\
\hline Jan & 31 & 995 & $11,391,905$ & 509,616 & $1,496,060$ & $9,386,229$ & $19,554,643$ \\
\hline Feb & 28 & 748 & $8,558,525$ & 707,710 & $1,351,280$ & $6,499,535$ & $13,540,697$ \\
\hline Mar & 31 & 583 & $6.671,894$ & $1,329,422$ & $1,496,060$ & $3,846,412$ & $8,013.359$ \\
\hline Apr & 30 & 162 & $1,854,576$ & $1,558,170$ & $1,447,800$ & $-1,151,394$ & 0 \\
\hline
\end{tabular}


Test 121590

$\mathrm{UA}=435 \mathrm{Btu} / \mathrm{hr}^{\circ} \mathrm{F}$

Heating System Efficiency $=0.46$

Table B.5

Predictions Using Test 121590 Data

\begin{tabular}{||l|c|c|c|c|c|c|c||}
\hline Month & Days & $\begin{array}{c}\text { Degree } \\
\text { Days }\end{array}$ & $\begin{array}{c}\text { Total Load } \\
\text { Btu }\end{array}$ & $\begin{array}{c}\text { Solar Gains } \\
\text { Btu }\end{array}$ & $\begin{array}{c}\text { Internal Gains } \\
\text { Btu }\end{array}$ & $\begin{array}{c}\text { Net Load } \\
\text { Btu }\end{array}$ & $\begin{array}{c}\text { Fuel Use } \\
\text { Btu }\end{array}$ \\
\hline Oct & 31 & 112 & $1,165,104$ & $1,028,022$ & $1,496,060$ & $-1,358,978$ & 0 \\
\hline Nov & 30 & 426 & $4,447,440$ & 563,330 & $1,447,800$ & $2,436,310$ & $5,296,325$ \\
\hline Dec & 31 & 710 & $7,411,356$ & 354,239 & $1,496,060$ & $5,561,057$ & $12,089,254$ \\
\hline Jan & 31 & 995 & $10,388,844$ & 509,616 & $1,496,060$ & $8,383,168$ & $18,224,278$ \\
\hline Feb & 28 & 748 & $7,804,944$ & 707,710 & $1,351,280$ & $5,745,954$ & $12,491,204$ \\
\hline Mar & 31 & 583 & $6,084,432$ & $1,329,422$ & $1,496,060$ & $3,258,950$ & 7,084574 \\
\hline Apr & 30 & 162 & $1,691,280$ & $1,558,170$ & $1,447,800$ & $-1,314,690$ & \\
\hline \hline Total 55.2 MMBtu & & & & & \\
\hline
\end{tabular}

Test 121790

$\mathrm{UA}=477 \mathrm{Btu} / \mathrm{hr}^{\circ} \mathrm{F}$

Heating System Efficiency $=0.42$

Table B-6

Predictions Using Test 121790 Data

\begin{tabular}{||l|c|c|c|c|c|c|c||}
\hline \multicolumn{1}{|c|}{ Month } & Days & $\begin{array}{c}\text { Degree } \\
\text { Days }\end{array}$ & $\begin{array}{c}\text { Total Load } \\
\text { Btu }\end{array}$ & $\begin{array}{c}\text { Soiar Gains } \\
\text { Btu }\end{array}$ & $\begin{array}{c}\text { Internal Gains } \\
\text { Btu }\end{array}$ & $\begin{array}{c}\text { Net Load } \\
\text { Btu }\end{array}$ & $\begin{array}{c}\text { Fuel Use } \\
\text { Bus }\end{array}$ \\
\hline Oct & 31 & 112 & $1,277,597$ & $1,028,022$ & $1,496,060$ & $-1,246,485$ & 0 \\
\hline Nov & 30 & 426 & $4,876,848$ & 563,330 & $1,447,800$ & $2,865,718$ & $6,823,137$ \\
\hline Dec & 31 & 710 & $8,126,935$ & 354,239 & $1,496,060$ & $6,276,636$ & $14,944,371$ \\
\hline Jan & 31 & 995 & $11,391,905$ & 509,616 & $1,496,060$ & $9,386,229$ & $22,348,164$ \\
\hline Feb & 28 & 748 & $8,558,525$ & 707,710 & $1,351,280$ & $6,499,535$ & $15,475,082$ \\
\hline Mar & 31 & 583 & $6,671,894$ & $1,329,422$ & $1,496,060$ & $3,846,412$ & $9,158,125$ \\
\hline Apr & 30 & 162 & $1,854,576$ & $1,558,170$ & $1,447,800$ & $-1,151,394$ & \\
\hline \hline Total 68,7 MMBtu & & & & & \\
\hline
\end{tabular}


Test 121990

$\mathrm{UA}=452 \mathrm{Btu} / \mathrm{hr}^{\circ} \mathrm{F}$

Heating System Efficiency $=0.41$

Table B.7

Predictions Using Test 121990 Data

\begin{tabular}{||l|c|c|r|r|r|r|r||}
\hline \multicolumn{1}{|c|}{ Month } & Days & $\begin{array}{c}\text { Degree } \\
\text { Days }\end{array}$ & $\begin{array}{c}\text { Total Load } \\
\text { Btu }\end{array}$ & $\begin{array}{c}\text { Solar Gains } \\
\text { Btu }\end{array}$ & $\begin{array}{c}\text { Internal Gains } \\
\text { Btu }\end{array}$ & $\begin{array}{c}\text { Net Load } \\
\text { Btu }\end{array}$ & $\begin{array}{c}\text { Fuel Use } \\
\text { Bu }\end{array}$ \\
\hline Oct & 31 & 112 & $1,210,637$ & $1,028,022$ & $1,496,060$ & $-1,313,445$ & 0 \\
\hline Nov & 30 & 426 & $4,621,248$ & 563,330 & $1,447,800$ & $2,610,118$ & $6,366,141$ \\
\hline Dec & 31 & 710 & $7,700,995$ & 354,239 & $1,496,060$ & $5,850,696$ & $14,269,990$ \\
\hline Jan & 31 & 995 & $10,794,845$ & 509,616 & $1,496,060$ & $8,789,169$ & $21,436,997$ \\
\hline Feb & 28 & 748 & $8,109,965$ & 707,710 & $1,351,280$ & $6,050,975$ & $14,758,474$ \\
\hline Mar & 31 & 583 & $6,322,214$ & $1,329,422$ & $1,496,060$ & $3,496,732$ & $8,528,616$ \\
\hline Apt & 30 & 162 & $1,757,376$ & $1,558,170$ & $1,447,800$ & $-1,248,594$ & \\
\hline \hline Total 65.4 MMBtu & & & & & \\
\hline
\end{tabular}

Test 122090

$\mathrm{UA}=540 \mathrm{Btu} / \mathrm{hr}^{\circ} \mathrm{F}$

Heating System Efficiency $=0.43$

Table B-8

Predictions Using Test 122090 Data

\begin{tabular}{||l|c|c|r|r|r|r|r||}
\hline \multicolumn{1}{|c|}{ Month } & Days & $\begin{array}{c}\text { Degree } \\
\text { Days }\end{array}$ & $\begin{array}{c}\text { Total Load } \\
\text { Btu }\end{array}$ & $\begin{array}{c}\text { Solar Gain } \\
\text { Btu }\end{array}$ & $\begin{array}{c}\text { Internal Gains } \\
\text { Btu }\end{array}$ & $\begin{array}{c}\text { Net Load } \\
\text { Btu }\end{array}$ & $\begin{array}{c}\text { Fuel Use } \\
\text { Btu }\end{array}$ \\
\hline Oct & 31 & 112 & $1,446,336$ & $1,028,022$ & $1,496,060$ & $-1,077,746$ & 0 \\
\hline Nov & 30 & 426 & $5,520,960$ & 563,330 & $1,447,800$ & $3,509,830$ & $8,162,395$ \\
\hline Dec & 31 & 710 & $9,200,304$ & 354,239 & $1,496,060$ & $7,350,005$ & $17,093,034$ \\
\hline Jan & 31 & 995 & $12,896,496$ & 509,616 & $1,496,060$ & $10,890,820$ & $25,327,488$ \\
\hline Feb & 28 & 748 & $9,688,896$ & 707,710 & $1,351,280$ & $7,629,906$ & $17,743,967$ \\
\hline Mar & 31 & 583 & $7,553,088$ & $1,329,422$ & $1,496,060$ & $4,727,606$ & $10,994,433$ \\
\hline Apr & 30 & 162 & $2,099,520$ & $1,558,170$ & $1,447,800$ & $-906,450$ & \\
\hline \hline Total 79.3 MMBtu & & & & & \\
\hline
\end{tabular}


Table B.10

Summary of Tully, NY, predictions

\begin{tabular}{|l|c|c|c||}
\hline Test & $\begin{array}{c}\text { UA } \\
\text { Bu/hroF }\end{array}$ & $\begin{array}{c}\text { Heating } \\
\text { Efficiency }\end{array}$ & $\begin{array}{c}\text { Fuel use } \\
\text { MMBu }\end{array}$ \\
\hline 121490 & 477 & 0.48 & 60.2 \\
\hline 121590 & 435 & 0.46 & 55.2 \\
\hline 121790 & 477 & 0.42 & 68.7 \\
\hline 121890 & 452 & 0.41 & 65.4 \\
\hline 121990 & 540 & 0.43 & 79.3 \\
\hline Mean & 476 & 0.44 & 65.8 \\
\hline Standard deviation & 36 & 0.03 & 8.2 \\
\hline Percent of mean & 7.5 & 5.9 & 12.5 \\
\hline
\end{tabular}


Winchester, MA, House

Solar predictions

Solar Area $=54 \mathrm{ft} 2$

tau $=0.7$

Table B.10

Solar Predictions for Winchester House

\begin{tabular}{||l|c|c|c|c|c|c||}
\hline \multirow{2}{*}{ Month } & \multirow{2}{*}{$\begin{array}{c}\text { Insolation } \\
\mathrm{MJ} / \mathrm{m}^{2}\end{array}$} & $\begin{array}{c}\text { Actual percent } \\
\text { sunshine at } \\
\text { airport }\end{array}$ & \multicolumn{2}{|c|}{$\begin{array}{c}\text { Estimated Daily Insolation on } \\
\text { Vertical Surface }\end{array}$} & \multicolumn{2}{|c|}{ Net Solar Gains } \\
\cline { 5 - 7 } & 18.97 & 0.65 & 13.39 & 1179.36 & 44579.9 & 0.490379 \\
\hline Jan & 19.64 & 0.53 & 11.32 & 996.53 & 37668.8 & 1.092396 \\
\hline Feb & 17.78 & 0.54 & 10.32 & 909.06 & 34362.4 & 1.065233 \\
\hline Mar & 12.44 & 0.59 & 7.87 & 693.16 & 26201.3 & 0.733638 \\
\hline Apr & & & & & & Btu/ft \\
\hline
\end{tabular}

Best estimate -- average of 5 tests

$\mathrm{UA}=420 \mathrm{Btw} / \mathrm{hr}^{\circ} \mathrm{F}$

Heating System Efficiency $=0.324$

Table B.11

Predictions Using Average UA and

Heating System Efficiency Estimates

\begin{tabular}{||l|c|c|c|c|c|c|c||}
\hline \multicolumn{1}{|c|}{ Month } & Days & $\begin{array}{c}\text { Degree Days } \\
\text { base-69 }\end{array}$ & $\begin{array}{c}\text { Total Load } \\
\text { Btu }\end{array}$ & $\begin{array}{c}\text { Internal Gains } \\
\text { Btu }\end{array}$ & $\begin{array}{c}\text { Solar Gains } \\
\text { Btu }\end{array}$ & $\begin{array}{c}\text { Net Load } \\
\text { Btu }\end{array}$ & $\begin{array}{c}\text { Fuel Use } \\
\text { Btu }\end{array}$ \\
\hline Jan & 11 & 459 & $4,626,720$ & $1,160,000$ & 490,379 & $2,976,341$ & $9,186,238$ \\
\hline Feb & 29 & 1149 & $11,581,920$ & $2,920,000$ & $1,092,396$ & $7,569,524$ & $23,362,728$ \\
\hline Mar & 31 & 1125 & $11,340,000$ & $2,940,000$ & $1,065,233$ & $7,334,767$ & $22,638,170$ \\
\hline Apr & 28 & 714 & $7,197,120$ & $2,370,000$ & 733,638 & $4,093,482$ & $12,634,204$ \\
\hline \hline
\end{tabular}


Test 120690

$\mathrm{UA}=389 \mathrm{Btu} / \mathrm{h}-\mathrm{F}$

Heating System Efficiency $=0.298$

Table B. 12

Predictions Using Test 120690 Data

\begin{tabular}{||l|c|c|c|c|c|c|c||}
\hline Month & Days & $\begin{array}{c}\text { Degree Days } \\
\text { base-69 }\end{array}$ & $\begin{array}{c}\text { Total Load } \\
\text { Btu }\end{array}$ & $\begin{array}{c}\text { Internal Gains } \\
\text { Btu }\end{array}$ & $\begin{array}{c}\text { Solar Gains } \\
\text { Btu }\end{array}$ & $\begin{array}{c}\text { Net Load } \\
\text { Btu }\end{array}$ & $\begin{array}{c}\text { Fuel Use } \\
\text { Btu }\end{array}$ \\
\hline Jan & 11 & 459 & $4,285,224$ & $1,160,000$ & 490,379 & $2,634,845$ & $8,841,762$ \\
\hline Feb & 29 & 1149 & $10,727,064$ & $2,920,000$ & $1,092,396$ & $6,714,668$ & $22,532,443$ \\
\hline Mar & 31 & 1125 & $10,503,000$ & $2,940,000$ & $1,065,233$ & $6,497,767$ & $21,804,587$ \\
\hline Apr & 28 & 714 & $6,665,904$ & $2,370,000$ & 733,638 & $3,562,266$ & $11,953,913$ \\
\hline \hline
\end{tabular}

Test 120790

$\mathrm{UA}=423 \mathrm{Btu} / \mathrm{hr}^{\circ} \mathrm{F}$

Heating System Efficiency $=0.334$

Table B.13

Predictions Using Test 120790 Data

\begin{tabular}{||l|c|c|c|c|c|c|c|}
\hline \multicolumn{1}{|c|}{ Month } & Days & $\begin{array}{c}\text { Degree Days } \\
\text { base-69 }\end{array}$ & $\begin{array}{c}\text { Total Load } \\
\text { Btu }\end{array}$ & $\begin{array}{c}\text { Internal Gains } \\
\text { Btu }\end{array}$ & $\begin{array}{c}\text { Solar Gains } \\
\text { Btu }\end{array}$ & $\begin{array}{c}\text { Net Load } \\
\text { Btu }\end{array}$ & $\begin{array}{c}\text { Fuel Use } \\
\text { Btu }\end{array}$ \\
\hline Jan & 11 & 459 & $4,659,768$ & $1,160,000$ & 490,379 & $3,009,389$ & $9,010,147$ \\
\hline Feb & 29 & 1149 & $11,664,648$ & $2,920,000$ & $1,092,396$ & $7,652,252$ & $22,910,934$ \\
\hline Mar & 31 & 1125 & $11,421,000$ & $2,940,000$ & $1,065,233$ & $7,415,767$ & $22,202,895$ \\
\hline Apr & 28 & 714 & $7,248,528$ & $2,370,000$ & 733,638 & $4,144,890$ & $12,409,850$ \\
\hline \hline Total 66.5 MMBtu
\end{tabular}

\section{Test 120890}

$\mathrm{UA}=420 \mathrm{Btu} / \mathrm{hr}^{\circ} \mathrm{F}$

Heating System Efficiency $=0.32$

Table B.14

Predictions Using Test 120890 Data

\begin{tabular}{||l|c|c|c|c|c|c|c||}
\hline \multicolumn{1}{|c|}{ Month } & Days & $\begin{array}{c}\text { Degree Days } \\
\text { base-69 }\end{array}$ & $\begin{array}{c}\text { Total Load } \\
\text { Btu }\end{array}$ & $\begin{array}{c}\text { Internal Gains } \\
\text { Btu }\end{array}$ & $\begin{array}{c}\text { Solar Gains } \\
\text { Btu }\end{array}$ & $\begin{array}{c}\text { Net Load } \\
\text { Btu }\end{array}$ & $\begin{array}{c}\text { Fuel Use } \\
\text { Btu }\end{array}$ \\
\hline Jan & 11 & 459 & $4,626,720$ & $1,160,000$ & 490,379 & $2,976,341$ & $9,301,066$ \\
\hline Feb & 29 & 1149 & $11,581,920$ & $2,920,000$ & $1,092,396$ & $7,569,524$ & $23,654,763$ \\
\hline Mar & 31 & 1125 & $11,340,000$ & $2,940,000$ & $1,065,233$ & $7,334,767$ & $22,921,147$ \\
\hline Apr & 28 & 714 & $7,197,120$ & $2,370,000$ & 733,638 & $4,093,482$ & $12,792,131$ \\
\hline \hline \multicolumn{1}{|l}{ Total 68.7 MMBtu } \\
\hline
\end{tabular}


Test 120990

$\mathrm{UA}=466 \mathrm{Btu} / \mathrm{hr}^{\circ} \mathrm{F}$

Heating System Efficiency $=0.246$

Table B.15

Predictions Using Test 120990 Data

\begin{tabular}{|l|c|c|c|c|c|c|c||}
\hline \multicolumn{1}{|c|}{ Month } & Days & $\begin{array}{c}\text { Degree Days } \\
\text { base-69 }\end{array}$ & $\begin{array}{c}\text { Total Load } \\
\text { Bru }\end{array}$ & $\begin{array}{c}\text { Internal Gains } \\
\text { Btu }\end{array}$ & $\begin{array}{c}\text { Solar Gains } \\
\text { Btu }\end{array}$ & $\begin{array}{c}\text { Net Load } \\
\text { Btu }\end{array}$ & $\begin{array}{c}\text { Fuel Use } \\
\text { Btu }\end{array}$ \\
\hline Jan & 11 & 459 & $5,133,456$ & $1,160,000$ & 490,379 & $3,483,077$ & $14,158,850$ \\
\hline Feb & 29 & 1149 & $12,850,416$ & $2,920,000$ & $1,092,396$ & $8,838,020$ & $35,926,911$ \\
\hline Mar & 31 & 1125 & $12,582,000$ & $2,940,000$ & $1,065,233$ & $8,576,767$ & $34,864,907$ \\
\hline Apr & 28 & 714 & $7,985,376$ & $2,370,000$ & 733,638 & $4,881,738$ & $19,844,463$ \\
\hline \hline Total 104.8 MMBtu
\end{tabular}

Test 121090

$\mathrm{UA}=402 \mathrm{Btu} / \mathrm{hr}^{\circ} \mathrm{F}$

Heating System Efficiency $=0.424$

Table B.16

Predictions Using Test 121090 Data

\begin{tabular}{|l|c|c|c|c|c|c|c||}
\hline \multicolumn{1}{|c|}{ Month } & Days & $\begin{array}{c}\text { Degree Days } \\
\text { base-69 }\end{array}$ & $\begin{array}{c}\text { Total Load } \\
\text { Btu }\end{array}$ & $\begin{array}{c}\text { Internal Gains } \\
\text { Btu }\end{array}$ & $\begin{array}{c}\text { Solar Gains } \\
\text { Btu }\end{array}$ & $\begin{array}{c}\text { Net Load } \\
\text { Btu }\end{array}$ & $\begin{array}{c}\text { Fuel Use } \\
\text { Btu }\end{array}$ \\
\hline Jan & 11 & 459 & $4,428,432$ & $1,160,000$ & 490,379 & $2,778,053$ & $6,552,012$ \\
\hline Feb & 29 & 1149 & $11,085,552$ & $2,920,000$ & $1,092,396$ & $7,073,156$ & $16,681,972$ \\
\hline Mar & 31 & 1125 & $10,854,000$ & $2,940,000$ & $1,065,233$ & $6,848,767$ & $16,152,752$ \\
\hline Apr & 28 & 714 & $6,888,672$ & $2,370,000$ & 733,638 & $3,785,034$ & $8,926,967$ \\
\hline \hline Total 48.3 MMBru
\end{tabular}


Table B.17

Summary of Winchester, MA results

\begin{tabular}{|l|c|c|c||}
\hline Test & $\begin{array}{c}\text { UA } \\
\text { Btw/hroF }\end{array}$ & $\begin{array}{c}\text { Heating } \\
\text { Efficiency }\end{array}$ & $\begin{array}{c}\text { Fuel use } \\
\text { MMBtu }\end{array}$ \\
\hline 120690 & 389 & 0.298 & 65.1 \\
\hline 120790 & 423 & 0.334 & 66.5 \\
\hline 120890 & 420 & 0.320 & 68.7 \\
\hline 120990 & 466 & 0.246 & 104.8 \\
\hline 121090 & 402 & 0.424 & 48.3 \\
\hline \hline Mean & 420 & 0.324 & 70.7 \\
\hline Standard deviation & 26 & 0.058 & 18.5 \\
\hline Percent of mean & 6.2 & 17.9 & 26.2 \\
\hline
\end{tabular}




\section{APPENDIX C}

SYNERTECH SYSTEMS CORPORATION FINAL REPORT 


\section{UNCERTAINTY ANALYSIS OF THE \\ MEASURED PERFORMANCE RATING SYSTEM}

Prepared for the NAHB National Research Center

under

NYSERDA Contract 1129-EEED-AEP-90

subcontract No. 2164.0002

This is a draft final report to which all readers are welcome to respond. Please share comments with its author, Dr. Michael stiles, at the address and phone below.

472-South Salina Street / Suite 410 / Syracuse. New York 13202-2401/ (315)422-3828 / FAX (315)422-6165 gò recrcled paper 


\section{INTRODUCTION}

The work that is the subject of this report was undertaken to assist the National Association of Home Builders Research Center (Research Center) in an uncertainty analysis by collecting utility bill data over at least one heating season and comparing it to MPR predictions for occupied houses.

Recently, the Measured Performance Rating (MPR) system had been previously used in two Geomet test houses with fair success (1). One of the primary questions investigated concerned the heat gains that are not directly measured by the MPR technique -- solar heat gain being foremost. The strategy was to carefully monitor the test structures and to use the fairly high resolution data thus obtained to estimate non-heating system heat gains. The Geomet houses were also ideal for supplying long-term results to compare with predictions based on MPR's short-term testing.

The MPR technique is also potentially applicable to the older housing stock typically receiving weatherization measures. Additional variables due to occupancy and other factors tend to complicate this application. Now that two years have passed since the MPR technique was used in a number of weatherization homes in upstate New York, many fuel bills have become available for postweatherization analysis. Detailed information on actual consumption is now available for comparison to MPR predictions about fuel consumption.

One of the implications of a successful application of the MPR technique of interest to the world of weatherization is the possibility of assessing the efficacy of the weatherization job at a given house with only a few days of tests. Ideally, the data needed to assess a weatherization job should be fairly simple to obtain and use. It was with this impetus that we undertook the development of a method for using easy-to-obtain data on average 
temperatures, wind speeds, and insolation in conjunction with the information gathered by an MPR test. The goal was then one of predicting heating fuel consumption for a period of a few months both before and after weatherization of a given house.

\section{METHODS}

Predictions of heating fuel consumption were attempted for two weatherized houses and one control house in upstate New York. Details of the methods for estimating heat gains not accounted for by the MPR technique were described in an earlier document prepared under this subcontract; this document is reproduced in Appendix A.

In summary, the following parameters were supplied for use in conjunction with the MPK test results:

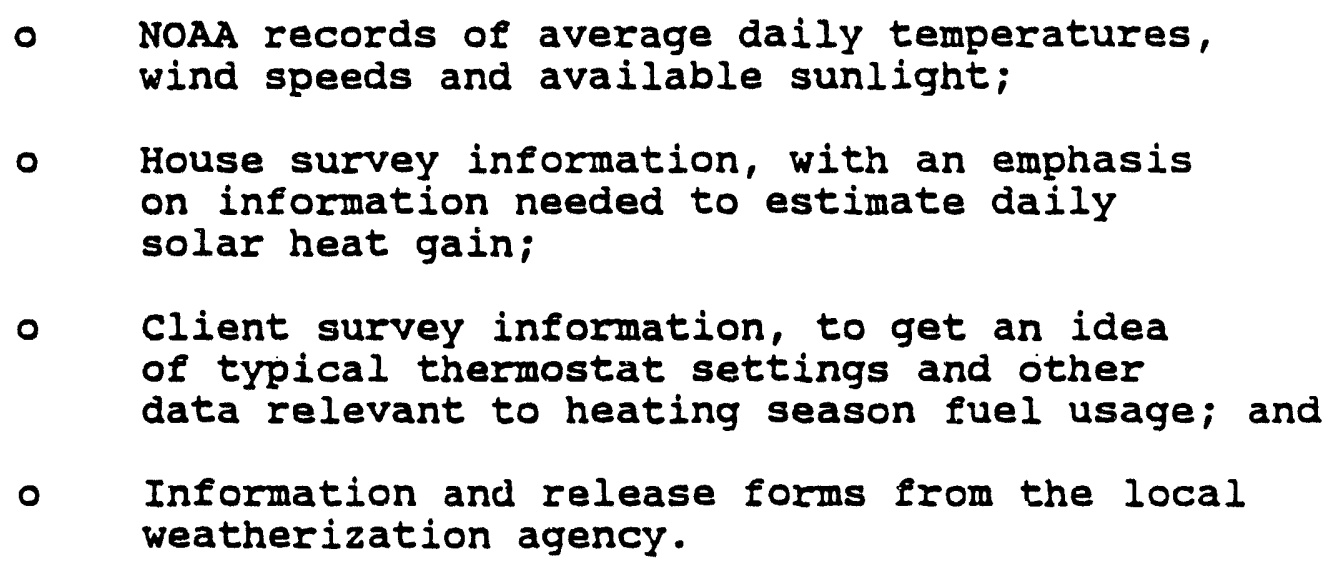

The house and client survey forms are reproduced in Appendix B.

There were sufficient numbers of fuel and electric bills from the period under consideration to benchmark pre- and postweatherization fuel consumption; only actual consumption information was used. PRISM was applied to this information. Estimates were attempted for entire heating-season trends in the houses, so that a "smoothed" trend in heating fuel use could be 
established in the form of a linear regression to the data. The best circumstance possible for analysis was thus a comparison between MPR predictions and values predicted by the linear trend assumed by PRISM (assuming that the data were well-described by a linear trend).

Results from PRISM analysis are presented in a slightly different format in this report. MPR predictions were made for specific months in both pre- and post-weatherization periods and were supplied in the form of Btu's needed for heating as a function of average outside temperature. PRISM data was thus re-plotted in this format in order to clearly compare its trends with those predicted by MPR. Additionally, the periods over which billing data were taken should be comparable to the periods over which MPR predictions of consumption were made. The MPR predictions were therefore grouped in periods of time equivalent to the average number of days in the corresponding billing periods plus or minus four days.

There are problems in many instances where the heating fuel consumption does not fit the linear model assumed by PRISM. In such cases, the slope and reference temperature resulting from PRISM analysis are suspect and cannot be used as reliable benchmarks. The data and their best linear fits are thus presented for such cases in this report, and the predictions from MPR are examined for whether or not they fall in the range of the billing data. More rigorous quantitative comparisons are not statistically applicable when the billing data do not fit a linear trend very well.

RESULTS

Weatherized Houses

The billing data from weatherized house \#1 are well described by 
the PRISM model in both the pre- and post-weatherization periods. House \#1 is heated by a gas furnace. Figure 1 shows a plot of the heating therms from the pre-weatherization billing data as well as the solid line based on PRISM's description of the trend. Also shown are two dashed lines, which demark the values that are within one standard error of estimation of the linear regression. The average number of days in the billing periods was 30.1 (range: 2333 days). Reference temperature was found to be 65.0 degrees (+13.7).

The asterisks in Figure 1 are MPR's predictions of consumption. It is seen that MPR's predictions of heating fuel requirements fall within the error bounds reasonable expected for the data; recall that the standard error of estimation is analogous to a standard deviation.

Similar data is plotted in Figure 2 for the post-weatherization period for house \#1. The solid line indicates PRISM's description of the linear trend in the data, and the dashed lines demark values within one standard error of estimation. The average number of days in the billing periods was 30.2 (range: 29-33). The reference temperature was found to be $65.3+/-2.1$ degrees, which is not significantly different from the pre-weatherization case; there is, however, a marked reduction in the slope. The MPR predictions in this case, however, fall short of the trend that is well described by PRISM's results. MPR's results are approximately within two standard errors of estimation in this case.

House \#2 is heated by an oil-fired furnace. There was no baseload to estimate in this case, because the furnace is dedicated solely to space heating. The billing data do not fit a linear trend very well, however. Pre- and post-weatherization results are shown in the graphs of Figures 3 and 4 , respectively. Average number of days in the pre-weatherization billing periods was 31.8 (range: 27-39), and the average number of days in the post-weatherization 
billing periods was 32.9 (range: 27-43).

The low R-squared values presented in Figures 3 and 4 indicate a wide scatter of the data points, and in both cases the apparent reference temperature was well above 80 degrees. It should be noted that the PRISM model of analysis does not apply very well to the data sets from this house, and so the apparently high reference temperature is extremely suspect.

Following ASHRAE, a Btu content of 1.4 therms/gallon of number two fuel oil was applied to the predictions of Btu values made by MPR in order to plot them on the same scales found in Figures 3 and 4 for the billing data. Two of the four MPR predictions fall within the general range of the raw billing data in the pre-weatherization case (Figure 3). The three MPR predictions fall within the general range of the raw billing data in the post-weatherization case (Figure 4). In the latter case, no attempt was made to compare the MPR predictions with the standard error of estimation because the apparent reference temperature was so high, which indicates a potential problem in trying to apply PRISM analysis (or even making assumptions about the linearity of the data).

The results from the control house are presented in Figure 5 . This house's gas furnace heating consumption was not well described by a linear trend. The average number of days in the billing periods was 61.3 (range: 59-63). In order to bring the periods of MPR's predictions into agreement with the billing data periods, MPR estimates were combined for the months of October and November, for the months of December and January and for the months of February and March.

Two of MPR's predictions fall within the general range of the raw billing data shown in Figure 5; one of the MPR values clearly overestimates consumption. MPR predicts, however, an apparent 
reference temperature in the range of about 50 degrees, which is reasonable but is not at all matched by the distribution of billing data in that range of average outdoor temperatures. The billing data may not fit a linear trend at all in this case, and one might expect the trend to asymptotically go to zero in the range of about 60-70 degrees.

One outlier was excluded from the billing data prior to PRISM analysis; this was a consumption of 515 therms for the months of March and April, when average outside temperature was 39.4 degrees. Excluding these values, the control house consumed 259.4 therms for heating (according to the actual billing data). MPR predicted a total consumption of 567.3 therms for heating for the same period (excluding March and April), an overestimation of 219\%. Clearly, this house was difficult to model with both PRISM and the MPR technique.

\section{DISCUSSION}

There was sporadic agreement between the fuel bill data and the predictions of consumption based on the MPR technique. There were no systematic differences in the departures of billing data and MPR predictions.

These findings point to several conclusions. First, there is no statistically rigorous method le.g., the standard error or estimation) for comparing MPR predictions to actual monthly consumption when the assumption of linearity does not hold. Second, the method of predicting consumption based on MPR does not consistently work very well when applied to month-long periods before and after weatherization. Third, neither PRISM nor MPR can always give reliable predictions of consumption when applied to periods of a month in duration. The poor performance of PRISM in certain cases reported here makes benchmarking consumption for 
purposes of an uncertainty analysis for MPR difficult.

There is the possibility that if higher-resolution data had been supplied for estimating extraneous heat gains, the MPR predictions might have been more in accord with the billing data. Such information is not, however, generally available to non-research personnel (such as typical weatherization professionals). We therefore recommend that the principles of MPR analysis be reexamined before the technique can be readily applied to houses undergoing weatherization.

Not the least among the problems encountered by MPR in predicting monthly consumption are occupancy during testing and the difficulties in estimating solar heat gain. These aspects of fuel consumption are notoriously difficult to model accurately with gross average data. Further development of the analytical and practical facets of the MPR technique will reveal whether it should be applied to the problems introduced in this report.

\section{REFERENCE}

(1) "Comparison of Two Building Thermal Test Methods," Final Project Report, Project No. 2146. NAHB National Research Center, 400 Prince George's Blvd., Upper Marlboro MD 20772-8731. May 1991. 


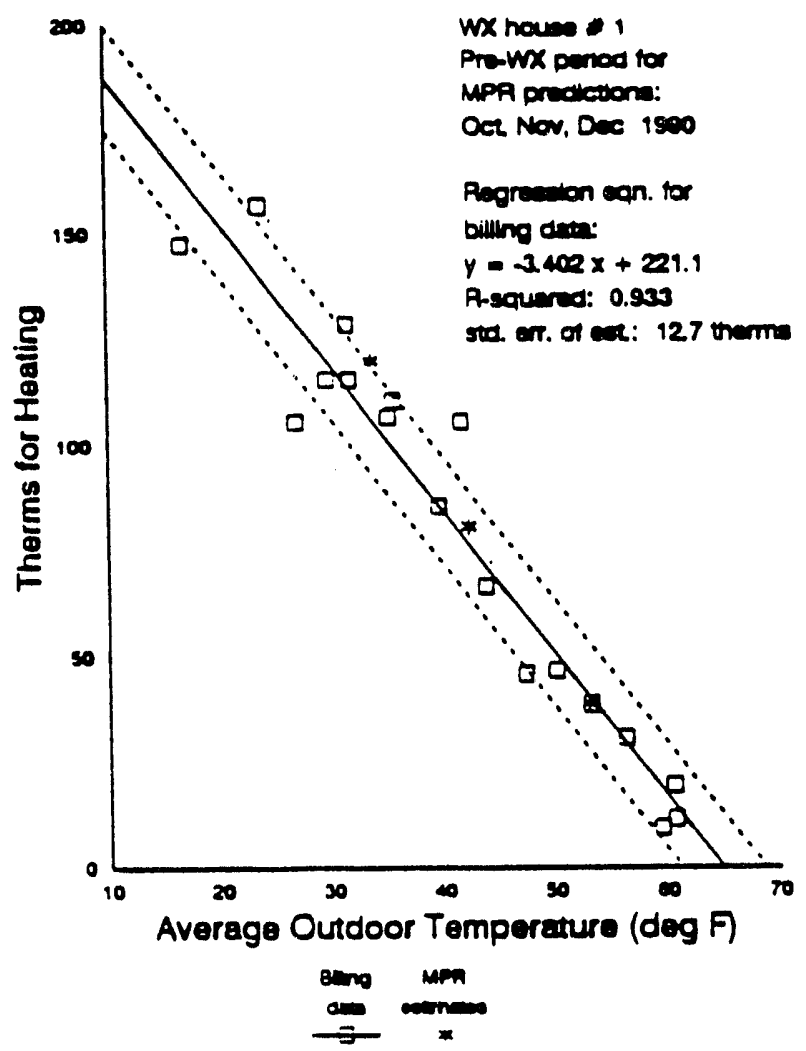

Figure 1.

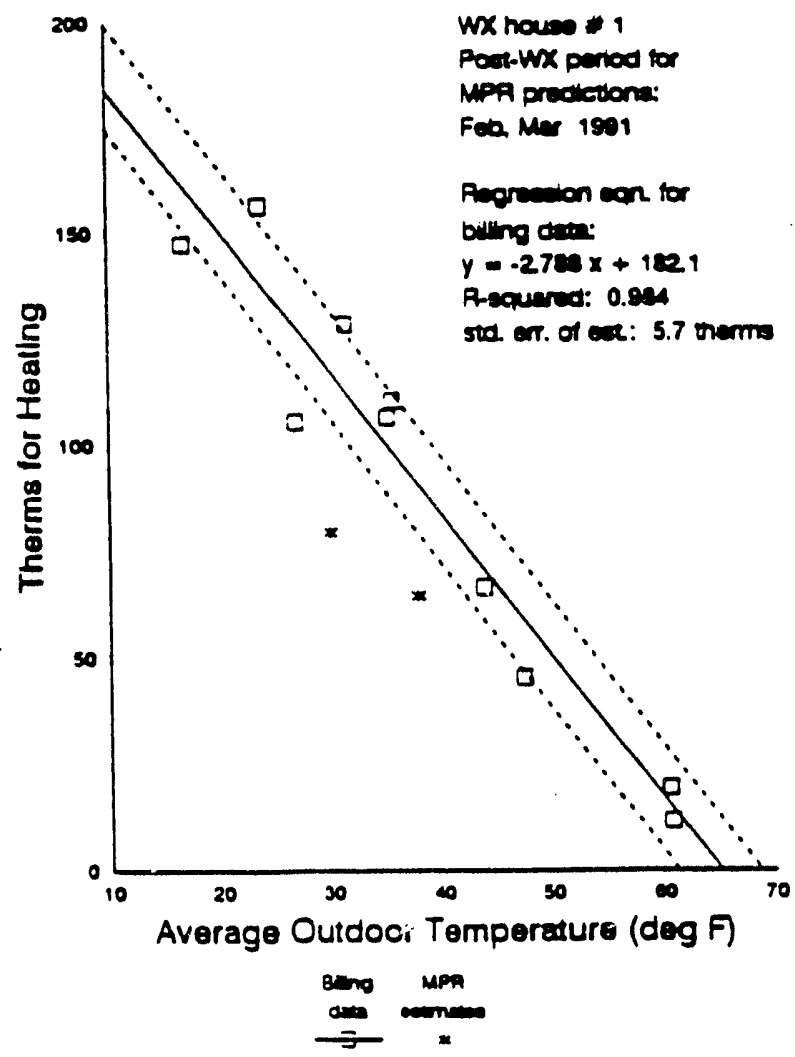

Figure 2. 


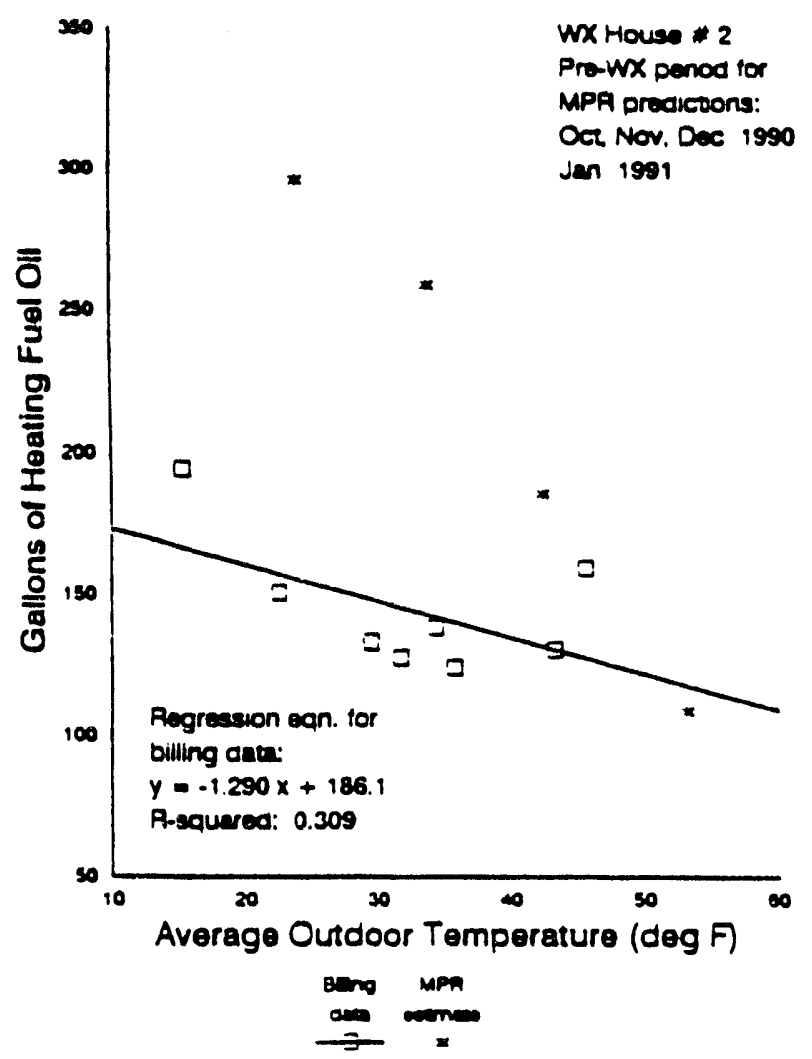

Figure 3.

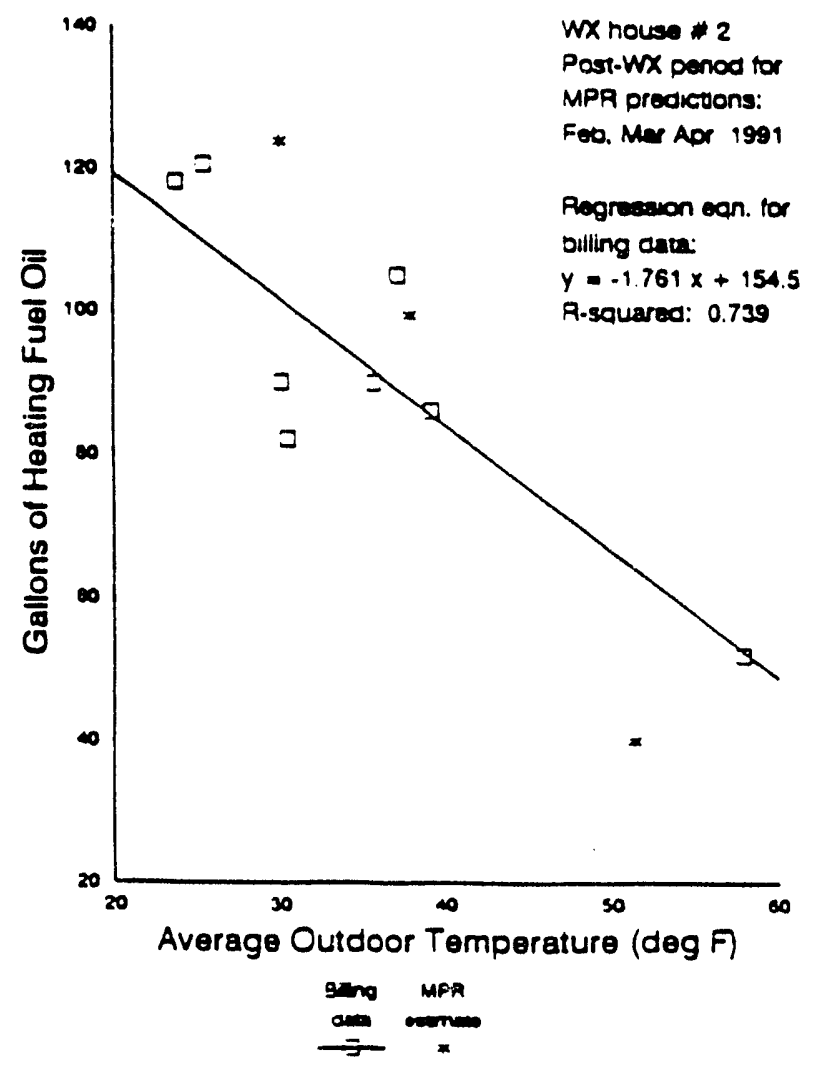

Figure 4. 


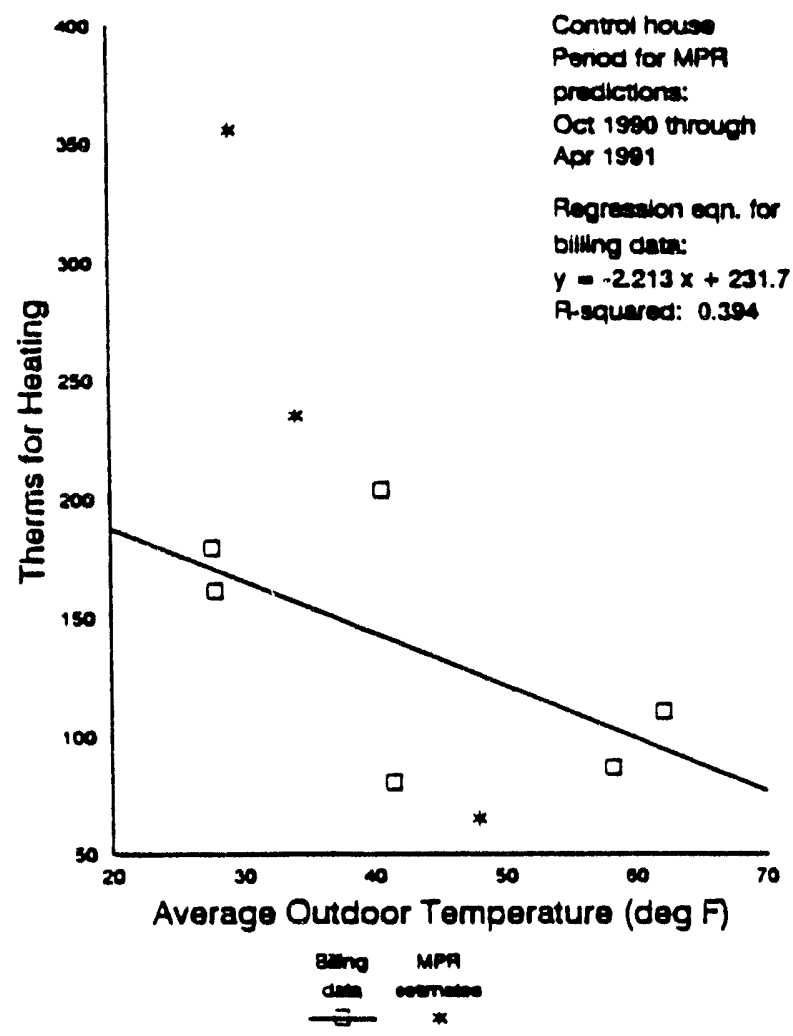

Figure 5. 
Appendix A

Heat Gains and Average Parameter Methods for MPR Analysis 
SWN TN 92-523

June 1992

\title{
TIME-AVERAGED HEAT GAINS AND TEMPERATURE PROFILES FOR MPR ESTMMATES OF FUEL CONSUMPTION
}

\author{
Prepared for the NABB Research Center \\ under NYSERDA Contract 1129-EEED-AEP-90 \\ Subcontract No. 2164.0002
}

Comments on this Technical Note should be directed to Dr. Michaei stiles at the address and phone number below. 


\author{
Time-Averaged Heat Gains and Temperature Profiles \\ for MPR Estimates of Fuel Consumption
Prepared for the NAHB Research Center by
Michael R. Stiles, Ph.D. Synertech Systems Corporation

\title{
Introduction
}

The MPR (Measured Performance Rating) technique has been designed to find the overall heat loss coefficient for a building. This Technical Note was prepared as part of MPR's characterization of weatherized houses in upstate New York. The goal of this phase of evaluation is to compare MPR estimates of heating season fuel consumption with those from other methods, e.g., PRISM, which have traditionally been applied to houses in weatherization programs. In particular, the MPR estimates of consumption over specific billing periods will be generated and compared to the actual consumption. MPR estimates of normalized annual consumption will be compared with comparable estimates from other methods, such as PRISM.

A number of interesting analytical features are introduced by using MPR on weatherized houses in upstate New York. The housing stock typically is of large old houses that have moderate to high thermal masses and moderate to substantial ground couplings. Heating effects due to occupancy must be taken into account. client behaviors must also be considered that may relate to the heating load demands of a house.

This Technical Note proposes the application of standard methods to estimate the factors that relate to the consumption of fuel as predicted by MPR analysis. The temporal resolution used for these factors in this phase of evaluation is that of the daily average. The principal factors that relate to heating demands include solar gains and gains due to occupancy and to appliance use. The losses due to conductive and convective processes, as estimated by MPR, can be expressed as functions of daily averages of temperature and wind velocities, respectively.

There are both advantages and disadvantages to using daily averaged estimates of the relevant variables. The advantages include the need for smaller databases than for computations based on hourly averages for the same period. The effects of unusual transient phenomena (such as a few hours of unusually windy weather) tend to be smoothed out in daily averages, which tends to simplify the details of analysis. The disadvantages arise if the predictive powers of a method of analysis decrease with decreasing data resolution. Thus, one important subtask in this study is to find whether daily averaged variables can be used in MPR analysis to

$$
\text { C. - . }
$$


predict consumption in occupied weatherized houses.

There are two basic issues that can examined, based on the information to be gathered in this study. The first issue is the degree to which MPR-based estimates of consumption for occupied weatherization houses agree with billing data throughout a given year's heating season. The second issue is the similarity among the estimates of normalized annual consumption that are based on MPR analysis, PRISM, and possibly other methods such as those afforded by furnace run-time data. Differences in methodologies for MPR analysis that are relevant to each of these issues are emphasized in the sections below.

Methods

\section{Direct Solar Gain}

The basic ain of this section is to apply standard principles of direct solar gain, such as those that are endorsed by ASHRAE. The detailed applications of these principles have been adapted from the text, solar Fngineering of Thermal processes by Duffie and Beckman. The method presented here for computing direct solar gain emphasizes the use of average daily estimates of the relevant variables.

We will consider heat gains across windows that face the most southerly for a given building (within 30 degrees of true south). A case can be made that windows facing other directions during winter in upstate New York contribute relatively little heat gain. The sun rises and sets at azimuth angles that are relatively far southeasterly and southwesterly, respectively.

Solar gain computations start with the statement of flow of energy across windows. There are gains and losses, with the loss terms already included in MPR's net heat loss coefficient. Heat gain is from solar radiation that takes the forms of direct beam, diffuse radiation and ground-reflected radiation.

The beam component is expressed as a function of average daily solar angle of incidence during a specific month. It is also a function of any overhang shading on windows. The diffuse component is assumed to be isotropic across the entire sky. The reflected component is assumed to be from both the beam and the diffuse sources combined.

The solar energy absorbed is proportional to the products of incident energy and net transmittance-absorptances. Absorptance is approximated for whole room-window configurations. Transmittances 
are taken as functions of window characteristics and averaged angles of incidence of the solar radiation.

The basic formulation of average daily solar gain through windows is taken from section 15.5 of Solar Engineering of Thermal processes. In a given month of the year, let the average daily radiation absorbed through a south-facing window be denoted as $\langle S\rangle$, having units of Joules/square meter/day. If the receiving area of window is $A$, then the average daily radiation absorbed by a room in the given month is:

$$
\begin{aligned}
& A_{I}\langle S\rangle=A_{I} \alpha\left\langle F_{\tau}\right\rangle\left(\left\langle f_{i}\right\rangle\left\langle\tau \tau_{b}\right\rangle\left\langle H_{b}\right\rangle\left\langle R_{b}\right\rangle+\right. \\
& \left\langle\tau \tau_{d}\right\rangle F_{I-s}\left\langle H_{d}\right\rangle+p\left\langle\tau q^{\rangle}\langle H\rangle / 2\right)
\end{aligned}
$$

The $\ll$ notation throughout Eqn. 1 denotes the average value of the quantity within. The terms of Eqn. 1 may be found from the following standard methods.

The effective absorptance of the combination of a room and its windows is $\underline{\alpha}$, and it can be approximated by:

$$
\alpha-1-\frac{\left\langle\tau_{b}\right\rangle \rho_{R} A_{I} / A_{R}}{1-\rho_{R}\left(1-A_{I} / A_{R}\right)}
$$

where $A_{R}$ is the room's surface area, $\left\langle\tau_{b}\right\rangle$ is the average value of solar beam transmittance (see below), and $\rho_{R}$ is the gross reflectance of the room's surfaces. The gross reflectance of interior surfaces of residences can be taken as an average midpoint value of the published ranges (Figure 10-2) in the IES Lighting Handbook, 1987 Applications Volume. A value of $\rho_{R}=0.5$ is thus obtained.

The monthly average radiation incident on a horizontal surface on a daily basis is $\langle\mathrm{H}\rangle$, which has units of Joules/square meter/day. $\langle\mathrm{H}\rangle$ has two components, which are $\left\langle\mathrm{H}_{\mathrm{b}}\right\rangle$ (beam radiation) and $\left\langle\mathrm{H}_{\mathrm{d}}\right\rangle$ (diffuse radiation), such that:

$$
\langle H\rangle=\left\langle H_{b}\right\rangle+\left\langle H_{d}\right\rangle
$$

The ratio $\left\langle\mathrm{H}_{\mathrm{d}}\right\rangle /\langle\mathrm{H}\rangle$ is usually taken as a function of the average monthly clearness index, $\left\langle\mathrm{K}_{\mathrm{T}}\right\rangle$.

For computing direct gain for normalized annual consumption: Use the tabulated values for upstate New York found in Table 1. 
For computing direct gain during an arbitrary billing period: Take the value of $<H>$ for the appropriate month from Table 1 , and instead of using the tabulated value of $\left\langle\mathrm{k}_{\mathrm{T}}\right\rangle$, use the value of percent available sunlight obtained from the National Weather Service for a given day and call it $R_{\mathrm{r}}$. Then compute the appropriate ratio $\left\langle H_{d}\right\rangle /\langle H\rangle$ for that day according to the following formulas:

$$
\begin{aligned}
& =0.99 \ldots \text { for } K_{T} \leq 0.17 \\
& =\left(1.188-2.272 K_{T}+9.473 K_{T}^{2}\right. \\
\left\langle H_{d}\right\rangle /\langle H\rangle & \left.-21.865 K_{T}^{3}+14.648 K_{T}^{6}\right) \text { for } 0.17<K_{T}<0.75 \\
& =-0.54 K_{T}+0.632 \ldots K_{T}<0.80 \\
& =0.2 \ldots \text { for } 0.75<K_{T} 20.80
\end{aligned}
$$

These formulas are taken directly from section 2.11 of Duffie and Beckman's text.

The control function, $\leq F_{0} \geq$, in Eqn. 1 , is set equal to unity if window insulation is used only when no solar radiation is incident on the southerly windows. If window insulators (e.g., heavy drapes) are used on a window on a sunny day during the heating season, $\left\langle F_{\mathrm{c}}\right\rangle$ is set to zero for that day.

The transmittance ( $\tau$ ) terms in Eqn. 1 are for the windows through which solar radiation enters the room. The three terms are for average transmittance of beam radiation $\left(\left\langle\tau_{b}\right\rangle\right)$, for diffuse radiation $\left(\left\langle\tau_{d}\right\rangle\right)$ and for radiation reflected from the ground $\left(\left\langle\tau_{z}\right\rangle\right)$. The term containing $\left\langle\tau_{z}\right\rangle$ in Eqn. 1 also contains a term for overall reflectance from the ground, 2 . Typical values for ground reflectance are usually given as:

$$
\begin{aligned}
& =0.2 & & \text { for no snow cover } \\
& =0.5 & & \text { for patchy snow cover } \\
& =0.7 & & \text { for fresh snow cover }
\end{aligned}
$$

The evaluation of transmittance is explained very clearly in Sections 5.1 through 5.4 of Duffie and Beckman's text. Transmittances may be found from either calculation or they may be read from the charts provided. Three additional pieces of information are needed, however. Transmittance evaluations require knowledge of the number of transmitting surfaces, their extinction coefficients and the average values of angles of incidence of sunlight.

The number of transmitting surfaces is simply the number of panes 
per window per room. Further characterization includes the composition of the panes (plastic or glass) and the approximate thicknesses of the panes. This information can be gathered during a detailed site audit.

Extinction coefficients can be looked up in standard tables once the composition of the panes is known. For example, the typical extinction coefficient for window glass is $30 \mathrm{~m}^{-1}$. Any special features of the windowpanes are to be noted during the site audit.

The monthly average angles of incidence of the solar radiation for a south-facing room, at $43^{\circ}$ latitude for upstate New York, are listed in Table 2. These values are for the average incident angles of the beam component, $\left\langle\theta_{b}\right\rangle$. Together with the information outlined above, the beam transmittance $\left(\left\langle\tau_{b}\right\rangle\right)$ may be evaluated.

Approximations given by Duffie and Beckman show that the average angle of incidence for diffuse and ground-reflected radiation at a vertical window is independent of the month and is constant at about $59^{\circ}$. Further, when evaluating the remaining transmittances, one may make the assumption that:

$$
\tau_{d}=\tau_{g}
$$

of the remaining terms in Eqn. 1, there are two that are functions of shading due to overhangs and eaves. The ratio of beam radiation received by a shaded window to the beam radiation received by an unshaded window is $\leq f_{i} \geq$. The window's view factor of radiation received from the sky is $F_{r-s}$. The evaluation of these terms from measured window geometry is straight forward and is given in Section 15.4 of the text by Duffie and Beckman. of course, any windows that are shaded by larje evergreen trees or other obstructing objects throughout the day will be excluded from the direct solar gain calculation.

The final term to be defined in Eqn. 1 is the ratio of beam radiation on a window to the beam radiation on a horizontal surface, $\left\langle R_{b}\right\rangle$. Monthly average values of $\left\langle R_{b}\right\rangle$ are listed for upstate New York (latitude 430) in Table 3 .

Eqn. 1 can be used to compute solar gains for any number of days within given months, or for a series of entire months. If an MIPRbased estimate of consumption is to be mide for an arbitrary billing period, the former situation applies for as many months as there are in the billing period. An estimate of normalized annual consumption requires the latter situation. 


\section{Gains Due to Occupancy}

Typical values of heat gains are usually attributed to occupancy of a house. The Air Conditioning Contractors of America recommend the following values: study

$300 \mathrm{Btu} / \mathrm{hr}$ per person occupying house during time period under

1200 Btu/hr per hour of average use of appliances

\section{Temperature and wind Profiles}

We are attempting consumption estimates based on average daily quantities. What will be supplied will be average daily temperatures, average daily wind velocities and average daily percentage of available sunlight. The latter two profiles will be obtained from the National weather Service, located at Hancock Airport in Syracuse, New York. Synertech has maintained a database of average daily temperatures for New York State since 1987.

The average daily temperature differential that drives heat loss can be found as a function of average outside temperature and the average thermostat setting in a particular house. Average daily infiltration losses can be estimated from average daily wind speeds. The use of average daily availability of sunlight in determining daily solar gains has been discussed above. 
In addition to providing weather profiles, the basic information needed to compute heat gains that are not directly available from the MPR method will be gathered by synertech. This information will be gathered from a number of surveys, which are outlined below.

House survey

Gross house dimensions (if not already recorded)

Photographs of house, emphasizing trees or other shading features

Compass orientation of most southerly face of house

Types of windows on most southerly face of house; number of panes/window; glass or plastic; approximate thicknesses of panes; other special or unique features

window dimensions, projection of overhangs for shading, spacings between windows and overhangs

Room surface area, for each room having windows facing south

Client survey

Permission and release documents, as needed Permission to do follow-up study Permission to secure and use fuel bills Fuel bills

Verification of client occupancy

Thermostat settings and patterns of setback

Number of occupants in house during period under analysis

Appliance survey, client-estimated number of hours of operation of appliances

Curtaining or other seasonal window-insulating practices

Seasonal installation and removal of storm windows

close-off patterns of rooms during heating season

Weatherization agency survey

Permission and release documents, as needed

Copies of audit; work as done; follow-up inspection

Records of elapsed-timer heating system usage (Hunter, etc.) 
TABLE 1

Average Daily Solar Radiation on a Horizontal Surface,

in Joules/ $\mathrm{m}^{2} /$ day, by Month and by Average Monthly

Clearness Index, $\left\langle k_{\mathrm{T}}\right\rangle$, for Upstate New York

\begin{tabular}{|c|c|c|c|c|}
\hline Month & $\left\langle\boldsymbol{K}_{\mathrm{T}}\right\rangle$ & $<\mathrm{H}>$ & $\left\langle\mathrm{H}_{d}\right\rangle$ & $\left\langle\mathrm{H}_{b}\right\rangle$ \\
\hline Jan & 0.33 & 4,370 & 3,802 & 568 \\
\hline Feb & 0.35 & 6,484 & 5,511 & 973 \\
\hline Mar & 0.39 & 10,105 & 7,882 & 2,223 \\
\hline Apr & 0.45 & 15,025 & 10,518 & 4,507 \\
\hline May & 0.46 & 17,908 & 12,536 & 5,372 \\
\hline Jun & 0.49 & 20,177 & 12,712 & 5,196 \\
\hline Jul & 0.50 & 19,948 & 12,368 & 7,580 \\
\hline Aug & 0.48 & 17.064 & 11,092 & 5,972 \\
\hline Sep & 0.46 & 13,225 & 9,258 & 3,967 \\
\hline oct & 0.43 & 6,822 & 6,440 & 2,382 \\
\hline Nov & 0.31 & 4,525 & $4, \cap 27$ & 498 \\
\hline Dec & 0.28 & 3,238 & 2,979 & 259 \\
\hline
\end{tabular}




\section{TABLE 2}

Average Monthly Angles of Incidence

of Solar Beam for Upstate New York

\begin{tabular}{|c|c|}
\hline Month & $\left\langle\theta_{b}\right\rangle$, Degrees \\
\hline Jan & 40 \\
\hline Feb & 48 \\
\hline Mar & 58 \\
\hline Apr & 65 \\
\hline May & 69 \\
\hline תuJ & 74 \\
\hline Jul & 73 \\
\hline Aug & 68 \\
\hline Sep & 61 \\
\hline oct & 52 \\
\hline Nov & 42 \\
\hline Dec & 36 \\
\hline
\end{tabular}


Month

$<R_{\boldsymbol{B}}>$

Jan

2.65

Feb

1.75

Max

1.05

Apr

0.55

May

0.30

Jun

0.22

Jul

0.25

Aug

0.42

Sep

0.82

oct

1.50

Nov

2.40

Dec

3.00

Values are taken from Figure 2.16.1(d) of Duffie and Becloman's text, for a latitude of $43^{\circ}$. 


\section{REFERENCES}

Duffie, J. A. and Beckman, W. A. Solar Engineering of Thermal Processes. Wiley and Sons, NY, 1980 .

Kaufman, J. E. (ed.). IES Lighting Handbook, 1987 Applications Volume. Illuminating Engineering Society of North America, NY, NY.

Load Calculation for Residential winter and summer Air conditioning (Manual J). Air Conditioning Contractors of America, Washington D.C., 1986 . 
Appendix B

Survey Forms 
client's Name:

Date:

Address:

1) Please tell me how many people lived in the house from the period just prior to weatherization through the past winter. $B e$ sure to include yourself and please explain any special circumstances (such as long-term guests and people moving).

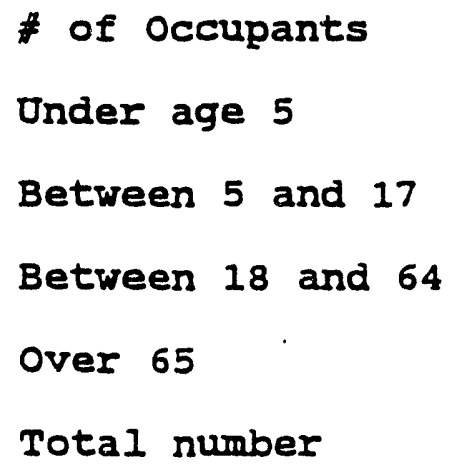

2a) During the heating season, do you keep the thermostat at one temperature setting, or do you change the setting? Fixed at degrees Changed it

2b) If you change the setting, what do you keep it at during the day while people are home?

What is the setting during the day when no one is at home? What is the setting at night, when you sleep? NOTES : 
3) Appliance use.

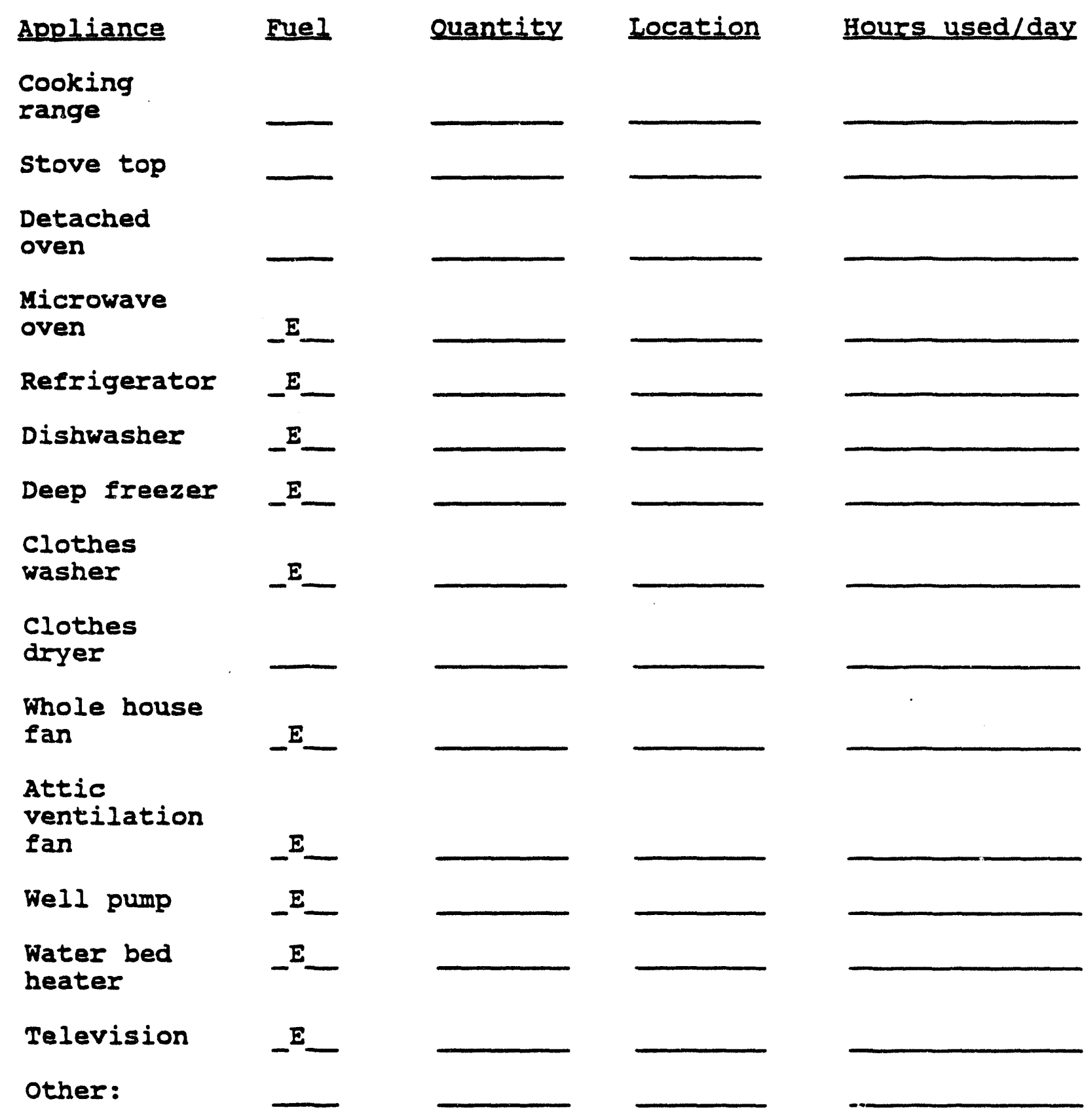

FUELS: NG natural gas; $P$ propane; 0 oil; $K$ kerosene; $E$ electricity; $W$ wood; $C$ coal; $x$ other

LOCATION: NH non-heated space; IH intentionally heated space; UH unintentionally heated space 
4) Curtaining and seasonal heating practice.

4a) Please describe briefly your household's seasonal windowinsulating practices, including use of curtains, plastic, or other insulation.

4b) Do you use storm windows during the heating season?

If yes, when approximately do you install them and when do you take them down?

Instail:

Remove:

4c) Do you close off any rooms during the heating season?

If yes, please describe: 
1) House plan. On a separate piece of paper, sketch out the floor plan, including dimensions.

Number of heated stories:

Height of building:

Is the basement heated?

Is the attic heated?

2) Solar admittance. Compass orientation of most southerly face of house:

(If one corner of the house faces due south within 20 degrees, supply the following information for both of the adjacent faces.)

For the southern face(s) of the house, sketch the windows and supply dimensions (height and width).

For each window on the southern face(s), list the composition of the window (glass, plexiglass, etc.) and approximate thickness (1/8" thickness is typical for glass).

For each window on the southern face(s), indicate the number of panes per window during the heating season.

Iist any other special or unique features of the southern windows.

4) Window overhangs. Refer to the accompanying diagram. For awning-style overhangs:

$$
\begin{aligned}
& P=\begin{array}{l}
\text { maximum distance that overhang extends away } \\
\text { from house }
\end{array} \\
& G=\begin{array}{l}
\text { gap between top of window glass and bottom } \\
\text { of overhang }
\end{array} \\
& H=\text { height of window glass } \\
& W=\text { width of window glass } \\
& E_{L}, E_{R}=\text { left and right extensions, respectively, } \\
& \text { between ends of overhang and ends of windows }
\end{aligned}
$$


For eave-style overhangs,

$P=$ maximum distance that eaves extend away from house

$G=$ gap between top of window glass and bottom of overhanging eaves

$\mathrm{H}=$ beight of window glass

4) Solar utilization. Room surface areas on the southern face(s) of the house for which there are windows. 
5) Photograph the sides of the house. Pay particular attention to any trees, houses or other obstructions near the southern face (s). Approximate the dimensions of any obstructions near the souther face (s).

NOTES on obstructions:

$\mathrm{C}_{\mathbf{3}} 31$ 


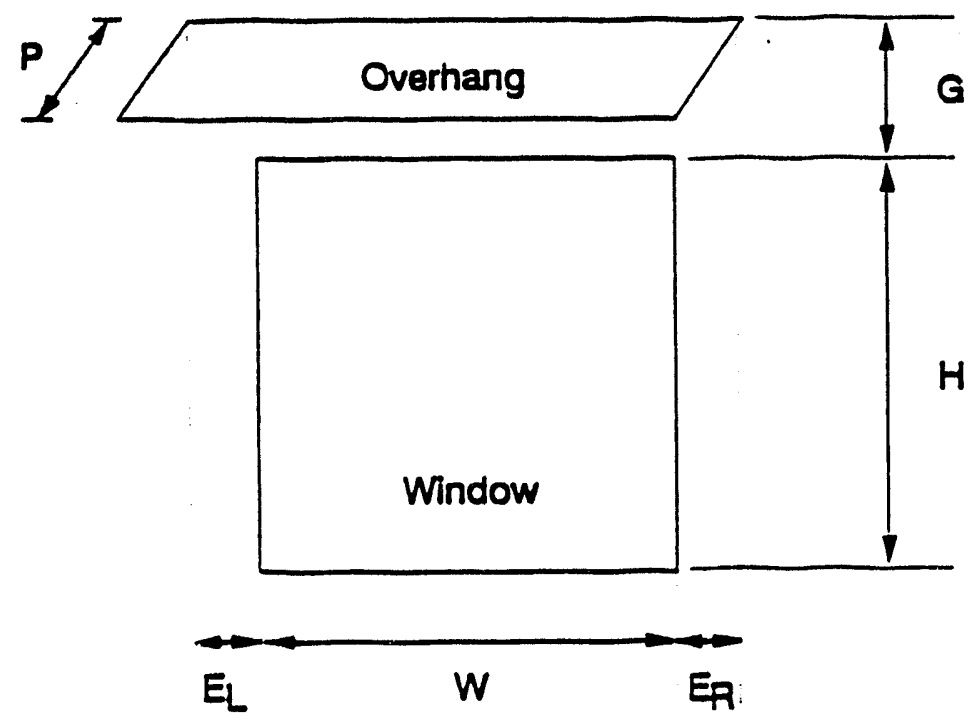

Measurements for awning-style overhangs
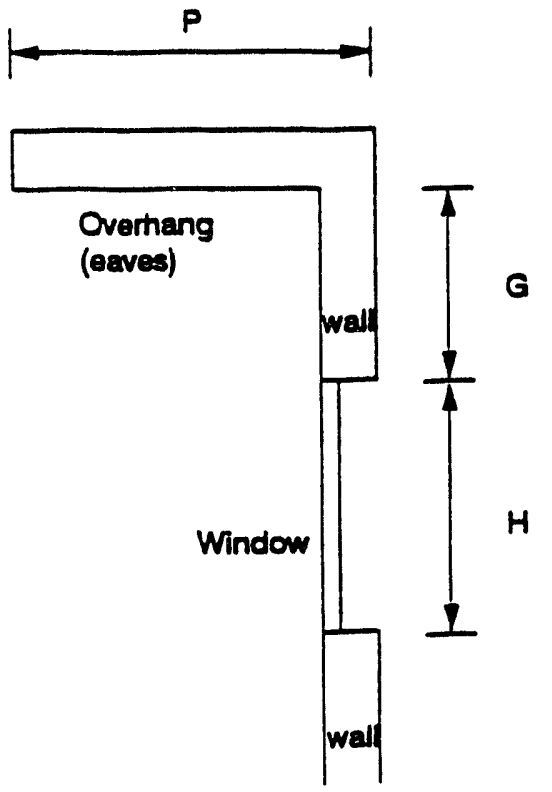

Measurements for eave-style overhangs 
APPENDIX D

J.J. DUFFY ASSOCIATES

FINAL REPORT 


\title{
ONCERTAINTY ANALYSIS OF THE MEABURED PERTORMANCE RATING BYBTEY
}

FINAL REPORT

By:

John Duffy and Brett Gouveia

J.J. Duffy Associates

8 orient st.

Winchester, MA $01890^{\circ}$

\author{
To: \\ NAHB Research Center \\ 400 Prince George's Blvd. \\ Upper Marlboro, MD 20772-9831
}

January 28, 1993 
CONTENTS

Section

1

INTRODUCTION

$1-1$

2

SIMULATION MODEL DESCRIPTION

$2-1$

3

EXPERIMENTAL DESIGN FOR SIMUIATION

TESTING MPR TESTS

$3-1$

4

WINCHESTER HOUSE MONITORING DATA

$4-1$

5

SUMMARY AND OBSERVATIONS

$5-1$

APPENDIX A. Bowie and winchester

Calibration Plots

$A-1$

APPENDIX B. Winchester House Monitoring Data

B-1

APPENDIX C. Simulation Programs

$c-1$

APPENDIX D. Miscellaneous Heating Season

Simulation Plots

$\mathrm{D}-1$ 


\section{INTRODUCTION}

The Measured Performance Rating (MPR) system has been in the process of development over the last several years $k Y J . J$. Duffy Associates, the University of Massachusetts Lowell, NAHB Research Center, and synertech under the sponsorship of the U.S. Department of Energy, the New York State Energy Research and Development Agency, and the Electric Power Research Institute (Duffy and Puri, 1985; Duffy and Saunders, 1987; NAHB/RC, 1991; Bourque and Duffy, 1992). The purpose of the MPR system is to provide an empirical measure of the thermal efficiency of small buildings. The MPR system involves the use of portable heaters, a blower door, temperature and electrical sensors, a data acquisition and control computer system in a one-night series of tests, after which relatively straightforward analyses are performed. The outcomes are estimates of the overall heat loss coefficient and the furnace system efficiency expected over a typical heating season.

The method is being developed for both predicting fuel usage for new homes and for measuring the effects of various weatherization procedures. One of the original design goals of the MPR method was that it would be suitable for use in a large number of buildings. Consequently, cost, speed, simplicity, and accuracy were important. The purpose of the present phase of development of the MFR approach is to analyze quantitatively the uncertainties in the estimates of the heat loss coefficients and furnace 
system efficiencies. In other words, we seek to answer the question: How much bias and scatter would we expect in a prediction of the heat loss coefficient and furnace system efficiency in any given MPR test and in a typical MPR test?

Two approaches were used to answer that question: (a) development and use of simulation models of two houses previously tested, and (b) collection of measured furnace fuel use during a heating season and comparison to the MPR predictions. The methods and results of these two approaches are reported in this document.

Although simulations have obvious drawbacks over the "real thing," there are three reasons (at least) for their use here: (a) they provide a cost-effective means of sensitivity and uncertainty analysis. (b) In addition, the field "worthiness" of the MPR method has been demonstrated, with over 100 field tests completed. (c) Also, there are very few (if any) existing houses for which the real heat loss coefficient and furnace system efficiency are known to a degree of precision greater than that of initial MPR estimates based on repeated tests.

The simulation models developed to characterize the winchester house and the Bowie house (on which several MPR tests were performed) is described in detail in section 2. With this model we have estimated the sensitivities of the heat loss coefficient and furnace system efficiency estimates to several key parameters, such as: varying outside temperatures, multizone heat transfer, basement ground loss and thermal mass, infiltration, mass in exterior walls, and effective sky temperature.

The experimental design approach that was used in this work is described in section 3. The results are also presented there.

The other approach to estimating the uncertainty in MPR predictions used in this study is that of comparing MPR predicted 
furnace fuel usage with measured fuel usage. The fuel data that we have gathered along with appropriate corresponding weather data for the winchester house is described and summarized in Section 4 .

Finally, some concluding remarks are given in section 5 .

\section{SECTION 1 REFERENCES}

Bourque, K., and J.J. Duffy, 1991, "Residential Thermal system Identification," Report to NAHB Research Center (also M.S. thesis of K. Bourque).

Bourque, K., and J.J. Duffy, 1992, "Building Thermal Parameter Estimation using system Identification and Short-Term Tests," Proceedings of the 1992 National Solar Energy Conference, American Solar Lnergy Society, Boulder, co.

Duffy, J.J., 1983, "Estimation of the Uncertainty in Class B Monitoring Results," Report to SERI, November.

Duffy, J.J., and H. Puri, 1985, "Extrapolation of short-Term Measurements to Long-Term Thermal Performance of Passive Buildings," Proceedings of the 10th National Passive Solar Conference, American Solar Energy Society, Boulder, $c o$.

Duffy, J.J., and D. Saunders, 1987, "Low-Cost Methods for Fvaluation of Space Conditioning Efficiency or Existing Homes," Univ. of Lowell report to NAHB Research Center.

Duffy, J.J., 1989, "Uncertainty in Passive Solar Performance Estimates," Proc. 14th National Passive Solar Conference, ASES, Boulder, co. 
Duffy, J.J., D. Saunders, and J. Spears, 1987, "Low-Cost Method for Evaluation of space Heating Efficiency of Existing Homes," Proc. 1987 Passive Solar Conf., ASES, Boulder, CO.

NAHB Research Center, 1991, "Measured Performance Rating system", NAHB/RC reports, Upper Marlboro, MD. 


\section{Section 2}

\section{SIMULATION MODEL DESCRIPTION}

By Brett Gouveia

\subsection{MODEL OVERVIEW}

Two models were constructed to simulate two houses tested in 1990 MPR tests. The buildings are located in Winchester, MA and Bowie,MD. The Winchester house is a typical older New England home built in the 1800 's. It is three stories with a basement. A central high efficiency gas furnace is located in the basement with heating ducts running throughout the house. The house has been weatherized with the addition of storm windows to the first and second floors, caulking to stop air leaks, and insulation added to the walls and attic. The Bowie house is a more modern style house that is two stories with an attached garage and heating ducts embedded in the first floor cement slab. Two out of the three upstairs rooms are closed off during the heating season. The first floor slab is not issulated around the perimeter.

The models approximating each building use a thermal network node map. A survey of the building construction was done and calculations of the thermal network parameters were made based on the ASHRAE estimates of building material resistance values. Each building was broken into nodes representing outside walls, inside walls, thermal mass within each room, basement floor and room air 
mass. Each zone or room is coupled to the adjacent zones and the outside. Heat transfer mechanisms modeled are radiation, convection, conduction and infiltration.

Schematics showing the Winchester and Bowie houses and the representative nodes are shown in Figures 2-1 (Bowie) and 2-8 (Winchester). Also included are Figures 2-2, 2-3 (Bowie) and Figure 2-9 (Winchester) indicating what physical locations in each house each node represents. A listing of the equations describing the relationships between the nodes for both houses can be found in Figures 2-6 through 2-7 (Bowie) and Figures 2-10 through 2-13 (winchester). Tables 2-1 (Bowie) and 2-2 (Winchester) are keys to the position each node has in the matrix.

MATLAB was used to implement this model. MATLAB is a matrix based mathematical tool. MATLAB was chosen because it allows easy manipulation of large groups of simultaneous equations and is well knowr by the authors.

The model is designed to use ambient dry bulb temperature, earth temperature, solar insolation, electric heaters or a central furnace and wind speed as inputs. The model outputs node temperatures, several heat loss coefficients, parameter test values, ambient temperature, and wind speed.

Each model is calibrated using test data from the previous MPR tests. Three calibrations are performed, coheat temperature, coheat energy balance, and the furnace efficiency and distribution.

\subsection{SIMULATION PROGRAM OVERVIEW}

The simulation is broken up into three files. The file init.m sets these initial temperatures for all nodes. The file prm.m 
contains all the resistance and capacitance values defining the thermal network. The file mtx.m builds the $A$ and $B$ matrices in the difference equation $X(t)=A X(t-1)+B U$ defining the relationships between the nodes. EQ3a.m or simu.m (Figure 2-14) performs the calculations and rebuilds the nonlinear portion of the thermal network equations at each time step. Eq3.m was used for calibration purposes and simu.m is configured for the parameter tests.

The simulation is broken up into half-minute time intervals for the winchester house and quarter-minute intervals for the Bowie house. These time steps were chosen for stability reasons. Both of these models were sensitive to room to room resistance values of less than 0.001 . As the coupling between rooms was increased the tendency for instability increased.

During each time step the node equations are solved simultaneously for the given set of inputs. The resulting node temperatures are then stored in data matrices. Another time step is then taken and new values calculated. Recalculating the system of equations each time step allows the non-linear terms to be linearized, increasing the accuracy of the simulation. The drawback of this method is it increases computer run time. At this time a one day simulation takes approximately 90 seconds on a $486 / 33 \mathrm{mHz}$ computer.

Inputs to the system are energy from a distributed forced air heating system or individual room electric heaters, outside air temperature, sky temperature, wind velocity (infiltration), and solar insolation.

The distributed forced air heating system during normal furnace operation is controlled by on-off thermostats simulated by a model developed by James kao et al. (1983). During the furnace efficiency test the furnace is controlled by an AD590 sensor. The time constant of the sensor incorporates the mixing time of the 
heater energy into the room and was determined during the calibration tests. During the coheat tests each node heater is controlled by a corresponding AD590 sensor.

Outside air temperature, wind velocity (infiltration), and direct incident solar is directly taken from a weather file constructed for this test using TMY files.

\subsection{MODEL DESCRIPTION}

This model assumes that the air space of any node is well mixed and therefore the same temperature throughout. In addition any heat inputs are assumed to be applied to the entire air mass evenly and instantaneously. The delay that occurs in the actual heating of $a \mathrm{r}$ rom is accounted for by increasing the time constant of the AD590 temperature sensor in the model.

Lumping the room air mass has drawbacks. This became apparent up in the calibratinn of the model. In the model all of the room air is at one temperature while in the reality it would be stratified. Because of stratification the data used to compare the model to is sensitive to location. For exampl?, in the winchester house the basement temperature was taken near the ceiling. Matching the model to this will force the model to heat the entire basement air to the higher ceiling temperature and thus use more energy than the data indicates. The second drawback is the temperature probe only sees the energy from the heaters after the air has time to mix. This delay doesn't exist with a lumped mass model. To compensate for this a 15 minute time constant was added to the model's AD590 temperature sensors, based on comparing the model temperature variations to the data's.

Infiltration is based on a model proposed by Sherman and Grimsrud (1980). This model predicts infiltration according to wind effects, stack effects and the ASHRAE's estimate of crack areas 
for varicus building parts such as windows, fireplaces, etc.. The wind effect varies linearly with wind speed and the stack effect is a function of the square root of the inside-outside temperature difference. The infiltration is calculated separately for each room. These numbers are later adjusted according to blower door results from each house. These equations are updated once an hour in the program to take into account the changes in the mentioned factors. It was felt that updating the infiltration or the radiation and solar increased computer time without any appreciable gain in accuracy.

Radiation is a function of the fourth power of the absolute temperature of the radiating surface and the sky temperature. since this is nonlinear, the radiation resistance was recalculated every hour based on new surface and sky temperatures. The sky temperature was taken to be 6 degrees Celsius below the air temperature according to a model by Anderson (1983). Radiation is calculated for the outside surface of the walls and the windows.

The solar insolation is input directly into the outside wall nodes and the interior room mass nodes. The energy through the windows is inputted into the interior room mass instead of the air directly to simulate the heating of the floor walls and furniture that would occur in the actual house. The horizontal insolation is first taken on an hourly basis from TMY files and converted to four vertical surfaces in the east, west, north and south directions and the roof. This is done in a separate program and then added to the weather data file. The simulation model then calculates the energy into the outside wall based on wall area and absorptivity. Energy through the windows into the interior rorn mass is calculated based on window area and transmissivity. The solar energy reaching the room air mass will have a time delay therefore based on the size of the interior room mass and the exterior wall mass. 
Convection was considered to be linear function of temperature. Values for horizontal and vertical convection coefficients were taken from the ASHRAE handbook and assumed to be constant. These were added in series with conduction components also taken from the ASHRAE handbook to get a resistance into and out of each interior and exterior wall.

Heat transfer between room and floors was modeled by estimating the thermal resistance using the ASHRAE Handbook, the wall/floor areas and construction. These numbers were then adjusted during the calibration of the model. If it appeared in the data that two rooms were strongly coupled the numbers for those rooms were reduced by a factor of up to 10. One drawback to these models is that the difference in heat transfer due to hot air rising up to the next floor but not the other way around is not recognized. It is assumed that heat transfer between floors is the same whether it is up or down.

\subsection{MODEL CALIBRATION}

The model is calibrated by comparing it to the actual house that it is attempting to represent. The computer models and their data are referred to as winchester and Bowie. Data was taken during earlier MPR tests at both of these sites.

Three calibrations are done to fine tune the model. These are the coheat temperature calibration, the coheat energy balance. and the furnace distribution and efficiency test. The models were originally constructed from knowledge of the building construction and the ASHRAE Handbook thermal resistance estimates. The model has electric heaters located in the same areas as during the MPR tests with the same wattage. Heater on-off times along with outside temperature and wind speed if available are input into the model from the original MPR data. 
In the coheat temperature profile test the computer model electric heater on-off times follow the MPR data. The MPR room temperature data is then plotted against the resulting model's room temperatures. The building parameters are adjusted to get the model temperature profile to look similar to the data. When every room or node is within one to two degrees and the trends look similar, then the next stage in calibration occurs. As was mentioned earlier, the AD590 time constant was set during this test. The AD590 time constant was increased until the temperature swings in the sensors matched those observed in the data. The time constant chosen was 15 minutes.

In the coheat energy balance test, the model is allowed to turn the electric heaters off and on, sijaulating control by AD590 sensors located in each room. This follows the MPR coheat test protocol. This time the total energy in BTUs is added up for each room for the model and the MPR data and compared. If the total house energy usage for the entire period of the test is within $5 \%$ then adjustment of the model parameters stops. The model parameters can be adjusted only slightly in this test in order to not disturb the results of the first test. After this test a check must be made to see that the temperature comparisons are still reasonably close.

The furnace is calibrated during the furnace efficiency and distribution test. Normal furnace operation is controlled by a thermostat located in one room of both the Winchester and Bowie houses. This is node two for the winchester house and node one for the Bowie house. During the furnace efficiency test the thermostat is replaced by an AD590 sensor in the MPR tests and in this model. The winchester house will be used as an example. since node two has the sensor, then the node 2 temperature will always coincide with the data unless the sensor in the model is set at the wrong temperature. The energy going to remaining nodes depend on the furnace on-off times set by node 2 and the percentage of the total furnace output that is allocated to each 
of them. The percentage or distribution is adjusted until all of the nodes is approximately the same number of degrees away from the data for that node. If all are several degrees above what the data indicates, then the total furnace output is increased by adjusting the furnace efficiency upward causing the sensor in node two to reduce the furnace on times. If all are below what the data indicates then the furnace efficiency is decreased causing the thermostat in node two to increase the furnace on times and thus the temperature in the nodes. This is done until the temperature profiles match.

\subsection{BOWIE CALIBRATION}

The Bowie house was calibrated to two sets of data taken during MPR testing i. the fall of 1990. The results of this calibration can be found in appendix $A$ of this report. Each section is lateled according to the test types mentioned above.

Looking at the Coheat Node temperature calibration, one can see that the test was run for a period of 16 hours: 13 hours of coheat followed by 3 hours of normal furnace operation. Nodes 1 and 2 follow the data within $1 / 2$ degree. Node 3 ramps up 2 degrees for the 13-hour period staying within 1 degree of the data. This phenomena would have occurred to an even greater degree except that a strong coupling was made between the first floor cement slab and the room air. In the Bowie house the entire first floor consists of a heated cement slab. During the calibration of the Bowie house model it became clear that the slab served to dampen out temperature fiuctuations.

In order for the model to match the data, a strong coupling between the room air and the floor was made. It also became clear that the slab was coupled to the outside air to some degree. There was some data on the temperature of the kitchen floor to indicate this. In the Bowie model the floor was originally broken up into three layers from top to bottom as seen in figure 2-1. As 
the calibration went on the bottom two layers got larger and in essence represented the earth. To represent the data, the middle layer was coupled to the outside air. The first floor heated slab was difficult to model since it is affected by ground temperature, outside air temperature, inside air temperature, its capacitance or size, and the quantity of energy flowing into it from the embedded heating ducts. Trying to balance all of these factors was difficult since data only existed for the temperature of the top of the slab in the kitchen area.

The kitchen floor temperature plot shows the model follows the data very closely except during the first four hours. The data seems to indicate that some heat was still going into the slab four hours into the coheat.

The coheat energy calibration plots show that the model follows the temperature profile of the data indicating the thermostat set point is correct. However, looking at the heater energy plots, one can observe the model does not ramp up while the data shows it should. This is more pronounced in node 3 . The ramping up in the data seems to mirror the ramping down of the slab temperature. The node 2 data shows the first four hours of hea-er energy is flat corresponding to the flat slab temperature profile. After four hours the heater output ramps up while the slab temperature ramps down, suggesting a strong coupling between the slab and the air temperature. For the entire 13 hour coheat test the energy predicted by the model was adjusted to within $+0.23 \%$ of the data.

The furnace distribution and efficiency calibration shows that nodes 1 through 3 are very close to the data after adjusting the furnace output and distribution. One discrepancy that does stand out is node 3 . The temperature swings shown in the data are around 1 degree while the model swings 2 to $2+1 / 2$ degrees. This would be worse except that the model puts $60 \%$ of the furnace energy going to node 3 into node 3 's room mass. This damps out 


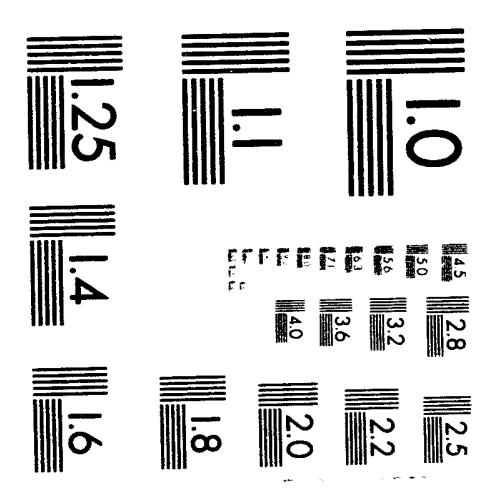



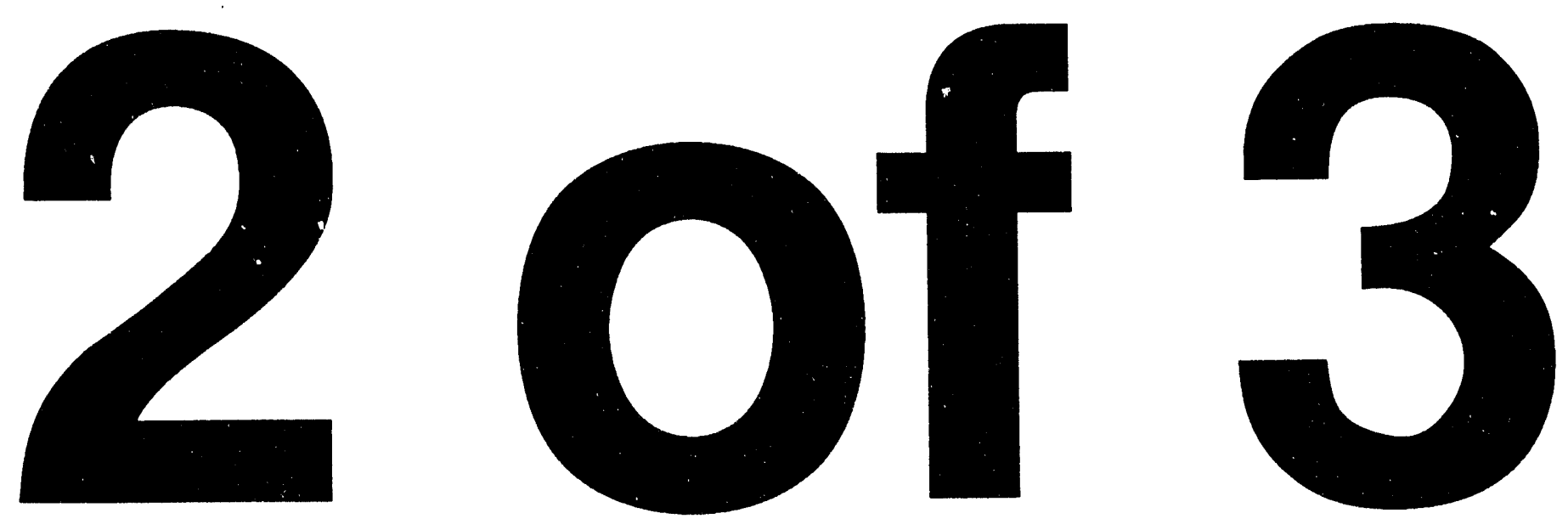
larger temperature swings to what they are now. This seems to say that node three should be getting little energy from the heating register and instead be getting more from nodes 1 and 2 . The way the model is currently set this will not work since node 3 is at $76 \mathrm{~F}$ while nodes 1 and 2 are at $74 \mathrm{~F}$ and $73 \mathrm{~F}$ respectively. This is a limitation of this model since it does not allow for a difference in energy transfer from downstairs to upstairs as opposed to the reverse.

\subsection{WINCHESTER CALIBRATION}

The winchester house does not have a heated slab but has a heated basement instead. The basement was heated to near the temperature of the upstairs thus minimizing any heat transfer between the two sections of the house. This made calibrating the winchester house a little more straightforward. All calibration plots for the Winchester house follow the Bowie house in Appendix $B$ and can be identified by the appropriate title.

From the winchester coheat temperature profile test nodes 1 through 3 are within $1 / 2$ degree of the data, nodes 4 and 5 are within 1.5 degrees and are dropping. Node 6 drops to 2 degrees below the data but then comes back up to follow the data closely. Notice that these tests are for a three hour period as opposed to the Bowie house tests of six hours. Initial conditions become more important in these shorter tests as the room doesn't have as much time to reach squilibrium. The set point of the initial temperatures of the wall masses did have a large effect on the calibration of this model. The rule was to set the interior wall mass temperatures to the same temperature as the room air.

In the coheat energy balance test, it appears that the node 1 AD590 set point for the furnace is $0.4 \mathrm{~F}$ too low. The temperature swings look the same. Node 2 follows the data very closely. Node 3 is approximately $1 / 2$ degree too low while node 4 is $1 / 2$ degree to high, indicating the furnace distribution is close but a 
little more energy could go to node 3 and a little less to node 4. Node 5 shows a 2 degree drop which is in contrast to a flat data temperature profile. This indicates that node 5 is losing too much heat or not gaining enough from the adjacent nodes. Node 6 also shows temperature fluctuations greater than the data.

overall, looking only at the upstairs reveals that the model uses 8.5\% more energy than the data indicates for the same period. The basement uses $11.65 \%$ more. This was by design since the basement was calibrated to a data sensor that was located at the ceiling. To actually heat the entire basement air mass to the ceiling temperature takes more energy.

The furnace distribution and efficiency calibration shows that nodes 1,3 and 4 are approximately 1 to 2 degrees too high. Node two is correct since the AD590 sensor is located in that node. Node 6 ramps up 6 degrees above the data. This indicates that the furnace efficiency is set a few percentage points too high but that it is close. Node 5 again shows that its temperature is not as stable as the data indicates it should be. The difference however is $t /$ - one degree.

There could be some further slight adjustments made for node 5's ramping up and down, for node 6's ramping, to adjust the furnace efficiency down and to adjust the model's energy usage down about 8.5\%. However time constraints necessitated that time would be better spent on other aspects of this project.

\subsection{RUNNING THE WINCHESTER AND BOWIE SIMULATIONS}

The simulation tests followed the sequence shown in figure 2-15. Yearly weather files were created from TMY data. These files contained the information shown in Table 2-3. Since we are only interested in outside temperatures where the furnace is operating, the middle part of the year containing the summer was omitted. The hourly horizontal solar data from the TMY file is 
converted to solar for vertical surfaces corresponding to the building walls. This data is then embedded in the control program ml.m that runs 24 hour simulations for the heating season. This control program calls the files shown, in the order shown in Fiqure 2-16. The control program calls the analysis program ANLZMPRD.exe last. After each 24 hour run it creates its own output file MPR. out (see Tables 2-4 and 2-5). The Winchester and Bowie houses differ slightly in the output parameters due to different building configurations. Run time for one heating season averaged five hours for the winchester model and eight hours for the Bowie model. 


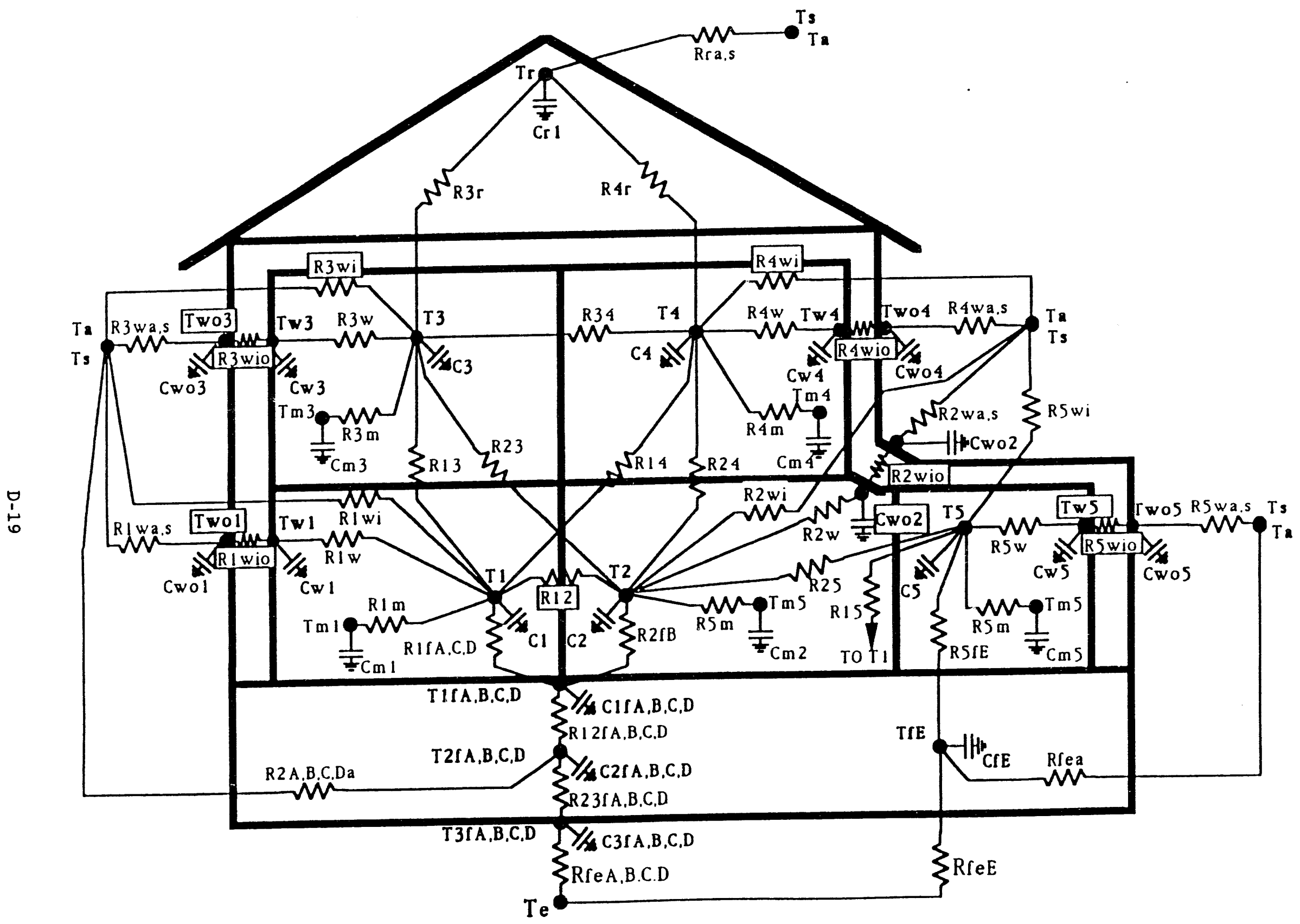

Figure 2-1 BOWIE HOUSE THERMAL NETWORK MODEL 

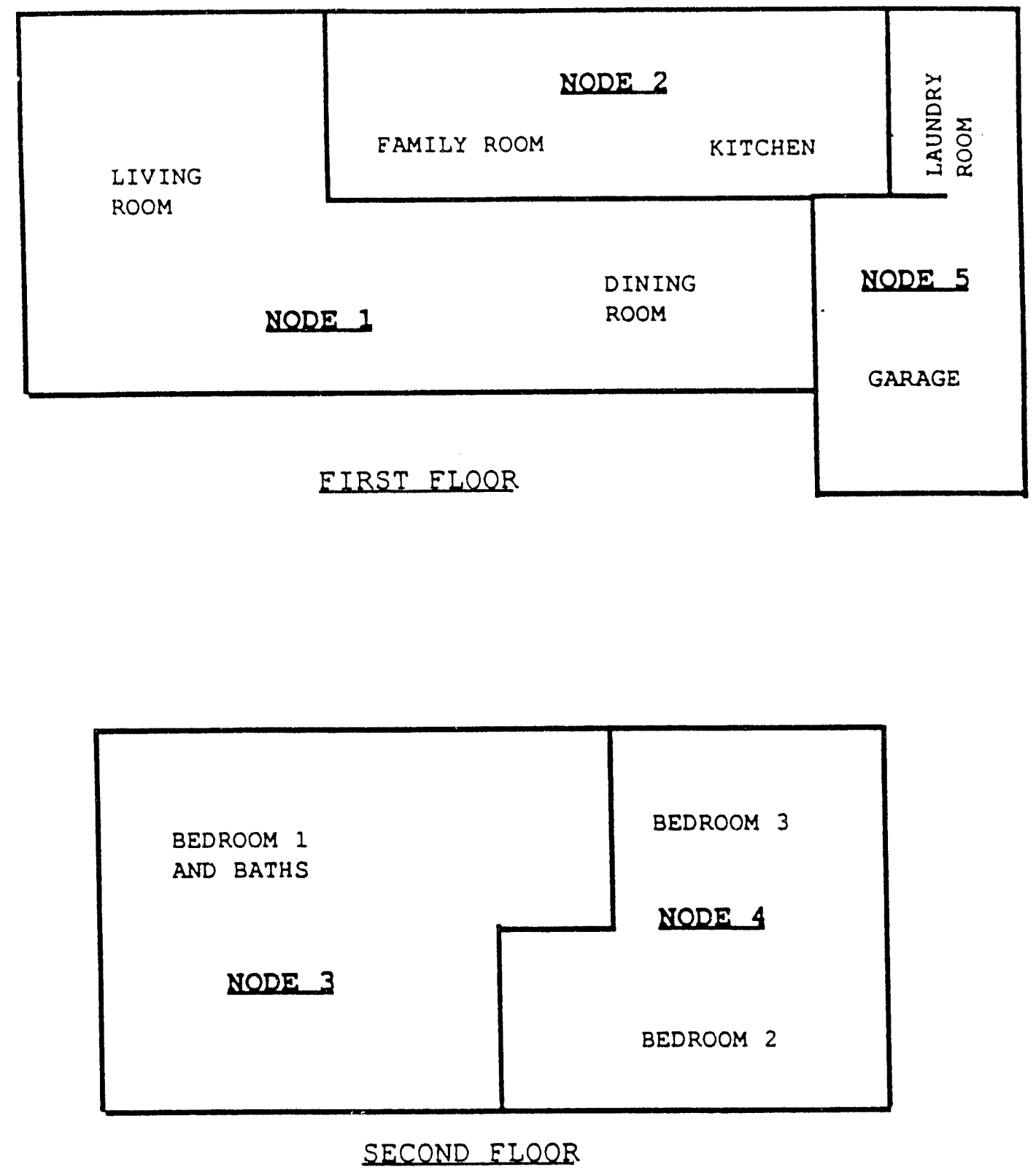

Eigure 2-2 BOWIE NODE LOCATIONS 


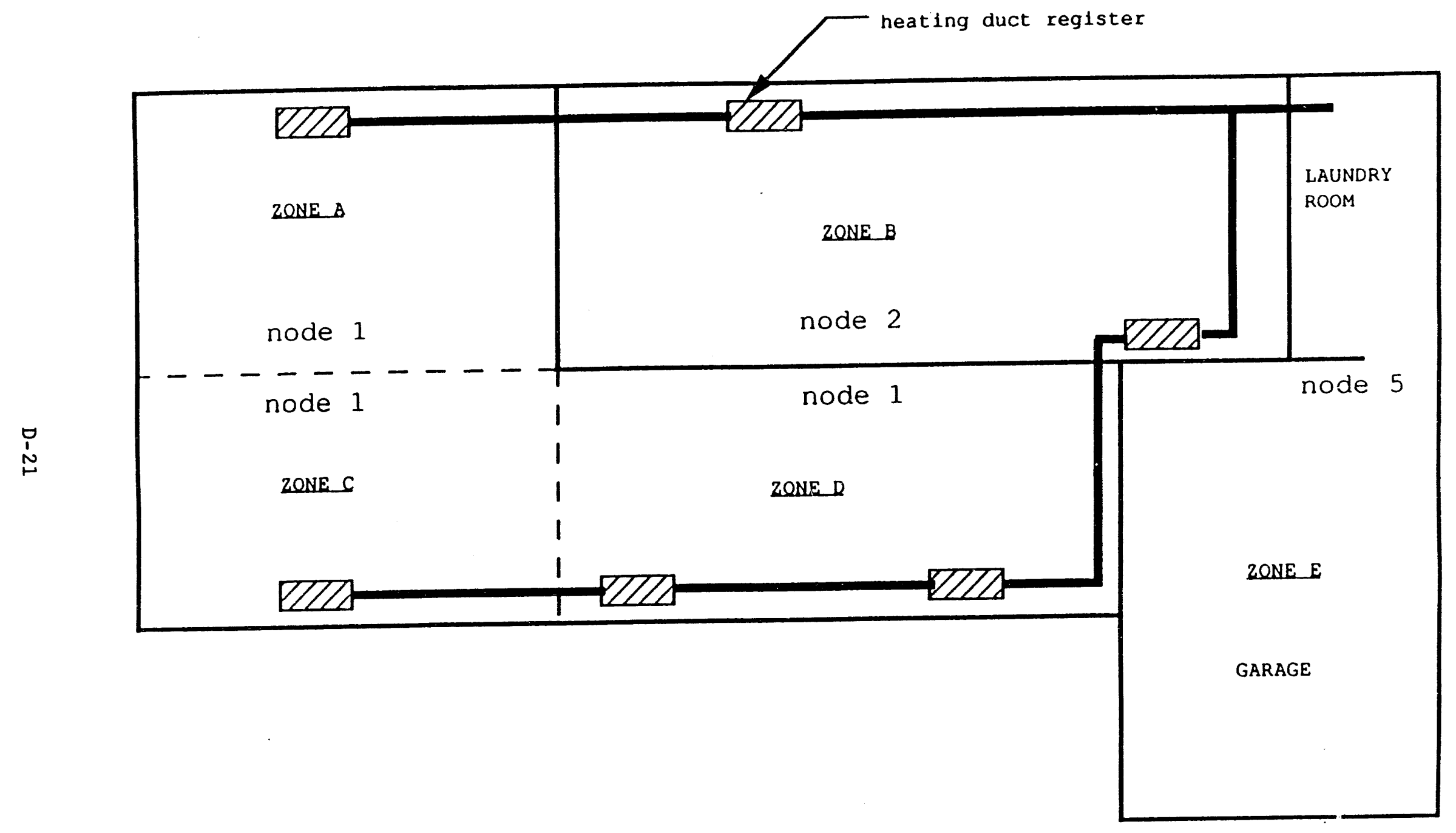

Figure 2-3 BOWIE HOUSE FIRST FLOOR HEATED SLAB 


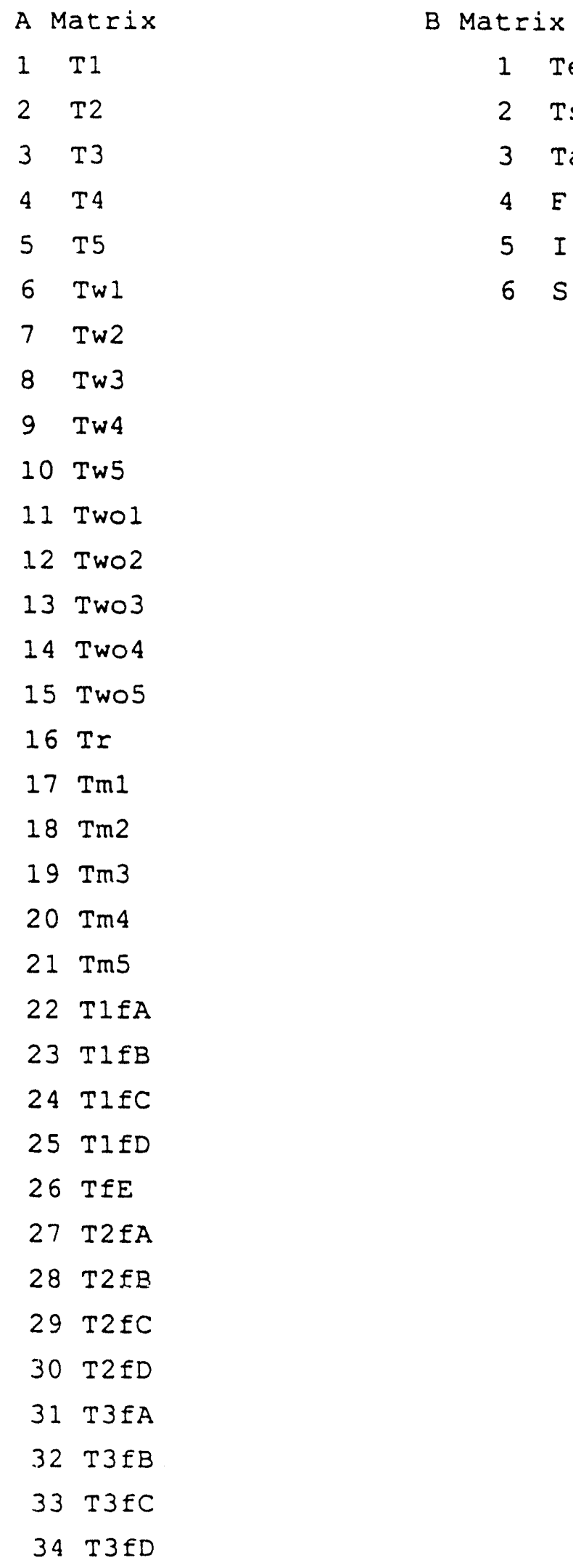




$$
\begin{aligned}
& \frac{\mathrm{dT}_{1}}{\mathrm{dt}}=\frac{-1}{\mathrm{C}_{1}}\left[\left(\frac{1}{\mathrm{R}_{12}}+\frac{1}{\mathrm{R}_{14}}+\frac{1}{\mathrm{R}_{13}}+\frac{1}{\mathrm{R}_{15}}+\frac{1}{\mathrm{R}_{1 \mathrm{fA}}}+\frac{1}{\mathrm{R}_{1 \mathrm{fC}}}+\frac{1}{\mathrm{R}_{1 \mathrm{D}}}+\frac{1}{\mathrm{R}_{1 \mathrm{a}}}+\frac{1}{\mathrm{R}_{1 \mathrm{~m}}}+\frac{1}{\mathrm{R}_{1 \mathrm{w}}}+\frac{1}{\mathrm{R}_{1 \mathrm{wi}}}+\frac{1}{\mathrm{R}_{1 \mathrm{ws}}}\right) \mathrm{T}_{1}\right. \\
& -\left(\frac{T_{2}}{R_{12}}+\frac{T_{4}}{R_{14}}+\frac{T_{3}}{R_{13}}+\frac{T_{5}}{R_{15}}+\frac{T_{f A}}{R_{1 E A}}+\frac{T_{f C}}{R_{1 f C}}+\frac{T_{f D}}{R_{11 D}}+\frac{T_{a}}{R_{1 a}}+\frac{T_{m 1}}{R_{1 m}}+\frac{T_{w 1}}{R_{1 w}}+\frac{T_{a}}{R_{1 w i}}+\frac{T_{s}}{R_{1 w s}}\right)-\left(F_{1}+S_{1}+I\right. \\
& \frac{\mathrm{dT}_{2}}{\mathrm{dt}}=\frac{-1}{\mathrm{C}_{2}}\left[\left(\frac{1}{\mathrm{R}_{12}}+\frac{1}{\mathrm{R}_{23}}+\frac{1}{\mathrm{R}_{24}}+\frac{1}{\mathrm{R}_{25}}+\frac{1}{\mathrm{R}_{2 B} \mathrm{~B}}+\frac{1}{\mathrm{R}_{2 \mathrm{a}}}+\frac{1}{\mathrm{R}_{2 \mathrm{~m}}}+\frac{1}{\mathrm{R}_{2 \mathrm{w}}}+\frac{1}{\mathrm{R}_{1 \mathrm{wi}}}+\frac{1}{\mathrm{R}_{2 \mathrm{ws}}}\right) \mathrm{T}_{2}\right. \\
& \left.-\left(\frac{T_{1}}{R_{12}}+\frac{T_{3}}{R_{23}}+\frac{T_{4}}{R_{24}}+\frac{T_{5}}{R_{25}}+\frac{T_{f B}}{R_{21 B}}+\frac{T_{a}}{R_{2 a}}+\frac{T_{m} 2}{R_{2 m}}+\frac{T_{w 2}}{R_{2 w}}+\frac{T_{a}}{R_{2 w i}}+\frac{T_{s}}{R_{2 w s}}\right)-\left(F_{2}+S_{2}+I_{2}\right)\right] \\
& \frac{\mathrm{dT}_{3}}{\mathrm{dt}}=\frac{-1}{\mathrm{C}_{3}}\left[\left(\frac{1}{\mathrm{R}_{23}}+\frac{1}{\mathrm{R}_{34}}+\frac{1}{\mathrm{R}_{13}}+\frac{1}{\mathrm{R}_{3 \mathrm{r}}}+\frac{1}{\mathrm{R}_{3 \mathrm{a}}}+\frac{1}{\mathrm{R}_{3 \mathrm{~m}}}+\frac{1}{\mathrm{R}_{3 \mathrm{w}}}+\frac{1}{\mathrm{R}_{3 \mathrm{wi}}}+\frac{1}{\mathrm{R}_{3} \mathrm{ws}_{\mathrm{s}}}\right) \mathrm{T}_{3}\right. \\
& \left.-\left(\frac{T_{2}}{R_{23}}+\frac{T_{4}}{R_{34}}+\frac{T_{1}}{R_{13}}+\frac{T_{r}}{R_{3 r}}+\frac{T_{a}}{R_{3 a}}+\frac{T_{m 3}}{R_{3 m}}+\frac{T_{w 3}}{R_{3 w}}+\frac{T_{2}}{R_{3 w i}}+\frac{T_{s}}{R_{3 w s}}\right)-\left(F_{3}+S_{3}+I_{3}\right)\right] \\
& \frac{\mathrm{dT}_{4}}{\mathrm{dt}}=\frac{-1}{\mathrm{C}_{4}}\left[\left(\frac{1}{\mathrm{R}_{14}}+\frac{1}{\mathrm{R}_{34}}+\frac{1}{\mathrm{R}_{24}}+\frac{1}{\mathrm{R}_{4 \mathrm{r}}}+\frac{1}{\mathrm{R}_{4 \mathrm{a}}}+\frac{1}{\mathrm{R}_{4 \mathrm{~m}}}+\frac{1}{\mathrm{R}_{4 \mathrm{w}}}+\frac{1}{\mathrm{R}_{4 \mathrm{wi}}}+\frac{1}{\mathrm{R}_{4 \mathrm{ws}}}\right) \mathrm{T}_{4}\right. \\
& \left.-\left(\frac{T_{1}}{R_{14}}+\frac{T_{3}}{R_{34}}+\frac{T_{2}}{R_{24}}+\frac{T_{r}}{R_{4 r}}+\frac{T_{2}}{R_{42}}+\frac{T_{m 4}}{R_{4 m}}+\frac{T_{w 4}}{R_{4 w}}+\frac{T_{2}}{R_{4 w i}}+\frac{T_{s}}{R_{4 w 5}}\right)-\left(F_{4}+S_{4}+I_{4}\right)\right] \\
& \frac{\mathrm{dT}_{5}}{\mathrm{dt}}=\frac{-1}{\mathrm{C}_{5}}\left[\left(\frac{1}{\mathrm{R}_{25}}+\frac{1}{\mathrm{R}_{15}}+\frac{1}{\mathrm{R}_{5 \mathrm{FE}}}+\frac{1}{\mathrm{R}_{5 \mathrm{a}}}+\frac{1}{\mathrm{R}_{5 \mathrm{~m}}}+\frac{1}{\mathrm{R}_{5 \mathrm{w}}}+\frac{1}{\mathrm{R}_{5 \mathrm{wi}}}+\frac{\mathrm{T}_{\mathrm{s}}}{\mathrm{R}_{\mathrm{ws}}}\right) \mathrm{T}_{\mathrm{s}}\right. \\
& \left.-\left(\frac{T_{2}}{R_{25}}+\frac{T_{1}}{R_{15}}+\frac{T_{f E}}{R_{S E E}}+\frac{T_{a}}{R_{s a}}+\frac{T_{m} 5}{R_{5 m}}+\frac{T_{w 5}}{R_{s w}}+\frac{T_{2}}{R_{s w i}}+\frac{T_{s}}{R_{s w s}}\right)-\left(F_{s}+S_{s}+I s\right)\right]
\end{aligned}
$$

Figure 2-4 BOWIE AIR MASS NODES 


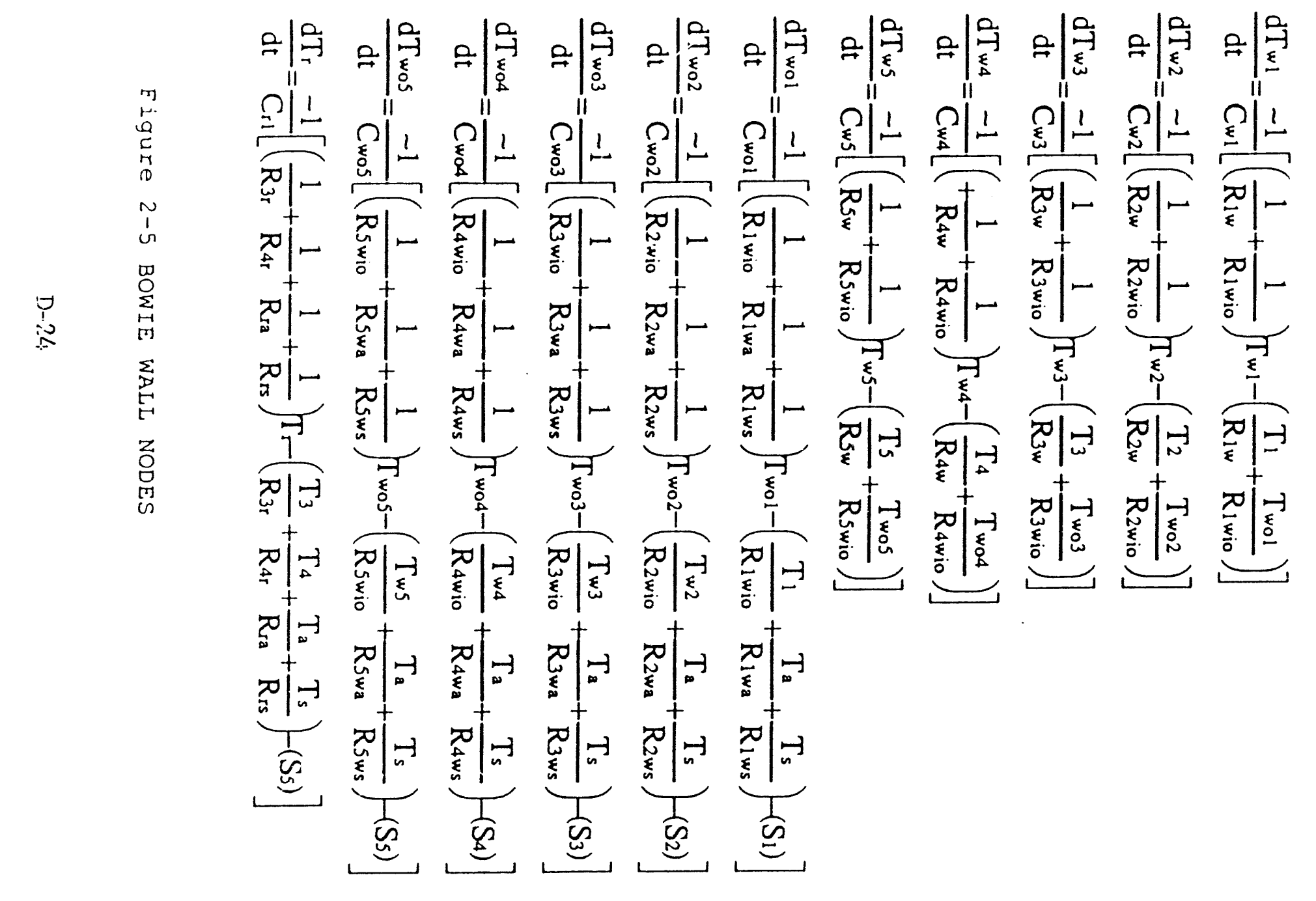




$$
\begin{aligned}
& \frac{\mathrm{dT}_{\mathrm{m} 1}}{\mathrm{dt}}=\frac{-1}{\mathrm{C}_{\mathrm{m} 1}}\left[\left(\frac{1}{\mathrm{R}_{1 \mathrm{~m}}}\right) \mathrm{T}_{\mathrm{m} 1}-\left(\frac{\mathrm{T}_{1}}{\mathrm{R}_{1 \mathrm{~m}}}\right)\right] \\
& \frac{\mathrm{dT}_{\mathrm{m} 2}}{\mathrm{dt}}=\frac{-1}{\mathrm{C}_{\mathrm{m} 2}}\left[\left(\frac{1}{\mathrm{R}_{2 \mathrm{~m}}}\right) \mathrm{T}_{\mathrm{m} 2}-\left(\frac{\mathrm{T}_{2}}{\mathrm{R}_{2 \mathrm{~m}}}\right)\right] \\
& \frac{\mathrm{dT}_{\mathrm{m} 3}}{\mathrm{dt}}=\frac{-1}{\mathrm{C}_{\mathrm{m} 3}}\left[\left(\frac{1}{\mathrm{R}_{3 \mathrm{~m}}}\right) \mathrm{T}_{\mathrm{m} 3}-\left(\frac{\mathrm{T}_{3}}{\mathrm{R}_{3 \mathrm{~m}}}\right)\right] \\
& \frac{\mathrm{dT}_{\mathrm{m} 4}}{\mathrm{dt}}=\frac{-1}{\mathrm{C}_{\mathrm{m}}}\left[\left(\frac{1}{\mathrm{R}_{4 \mathrm{~m}}}\right) \mathrm{T}_{\mathrm{m} 4}-\left(\frac{\mathrm{T}_{4}}{\mathrm{R}_{4 \mathrm{~m}}}\right)\right] \\
& \frac{\mathrm{dT}_{\mathrm{m} s}}{\mathrm{dt}}=\frac{-1}{\mathrm{C}_{\mathrm{m} s}}\left[\left(\frac{1}{\mathrm{R}_{5 \mathrm{~m}}}\right) \mathrm{T}_{\mathrm{ms}}-\left(\frac{\mathrm{T}_{\mathrm{s}}}{\mathrm{R}_{5 \mathrm{~m}}}\right)\right]
\end{aligned}
$$

Figure 2-6 BOWIE THERMAL MASS NODES 


$$
\begin{aligned}
& \frac{\mathrm{dT}_{1 \mathrm{FA}}}{\mathrm{dt}}=\frac{-1}{\mathrm{C}_{1 \mathrm{FA}}}\left[\left(\frac{1}{\mathrm{R}_{1 \mathrm{FA}}}+\frac{1}{\mathrm{R}_{12 \mathrm{FA}}}\right) \mathrm{T}_{1 \mathrm{EA}}-\left(\frac{\mathrm{T}_{1}}{\mathrm{R}_{1 \mathrm{IA}}}+\frac{\mathrm{T}_{2 \mathrm{fA}}}{\mathrm{R}_{12 \mathrm{FA}}}\right)\right] \\
& \frac{\mathrm{dT}_{1 \mathrm{FB}}}{\mathrm{dt}}=\frac{-1}{\mathrm{C}_{1 \mathrm{BB}}}\left[\left(\frac{1}{\mathrm{R}_{2 \mathrm{FB}}}+\frac{1}{\mathrm{R}_{12 \mathrm{BB}}}\right) \mathrm{T}_{1 \mathrm{BB}}-\left(\frac{\mathrm{T}_{2}}{\mathrm{R}_{2 \mathrm{FB}}}+\frac{\mathrm{T}_{2 \mathrm{FB}}}{\mathrm{R}_{12 \mathrm{BB}}}\right)\right] \\
& \frac{\mathrm{dT}_{1 \mathrm{fC}}}{\mathrm{dt}}=\frac{-1}{\mathrm{C}_{1 \mathrm{fC}}}\left[\left(\frac{1}{\mathrm{R}_{1 \mathrm{fC}}}+\frac{1}{\mathrm{R}_{12 \mathrm{fC}}}\right) \mathrm{T}_{1 \mathrm{fC}}-\left(\frac{\mathrm{T}_{1}}{\mathrm{R}_{1 \mathrm{fC}}}+\frac{\mathrm{T}_{2 \mathrm{fC}}}{\mathrm{R}_{12 \mathrm{fC}}}\right)\right] \\
& \frac{\mathrm{dT}_{1 \mathrm{DD}}}{\mathrm{dt}}=\frac{-1}{\mathrm{C}_{1 \mathrm{D}}}\left[\left(\frac{1}{\mathrm{R}_{1 \mathrm{D}}}+\frac{1}{\mathrm{R}_{12 \mathrm{D}}}\right) \mathrm{T}_{1 \mathrm{D}}-\left(\frac{\mathrm{T}_{1}}{\mathrm{R}_{1 \mathrm{D}}}+\frac{\mathrm{T}_{2 \mathrm{D}}}{\mathrm{R}_{12 \mathrm{D}}}\right)\right] \\
& \frac{\mathrm{dT}_{\mathrm{fE}}}{\mathrm{dt}}=\frac{-1}{\mathrm{C}_{\mathrm{EE}}}\left[\left(\frac{1}{\mathrm{R}_{S \mathrm{FE}}}+\frac{1}{\mathrm{R}_{\mathrm{feE}}}\right) \mathrm{T}_{\mathrm{fE}}-\left(\frac{\mathrm{T}_{\mathrm{S}}}{\mathrm{RSFE}_{\mathrm{SE}}}+\frac{\mathrm{T}_{\mathrm{e}}}{\mathrm{R}_{\mathrm{feE}}}\right)\right] \\
& \frac{\mathrm{dT}_{2 \mathrm{fA}}}{\mathrm{dt}}=\frac{-1}{\mathrm{C}_{2 \mathrm{fA}}}\left[\left(\frac{1}{\mathrm{R}_{12 \mathrm{fA}}}+\frac{1}{\mathrm{R}_{23 \mathrm{FA}}}+\frac{1}{\mathrm{R}_{2 \mathrm{fAa}}}\right) \mathrm{T}_{2 \mathrm{fA}}-\left(\frac{\mathrm{T}_{1 \mathrm{fA}}}{\mathrm{R}_{12 \mathrm{fA}}}+\frac{\mathrm{T}_{3 \mathrm{fA}}}{\mathrm{R}_{23 \mathrm{FA}}}+\frac{\mathrm{T}_{\mathrm{a}}}{\mathrm{R}_{2 \mathrm{fAa}}}\right)\right]
\end{aligned}
$$

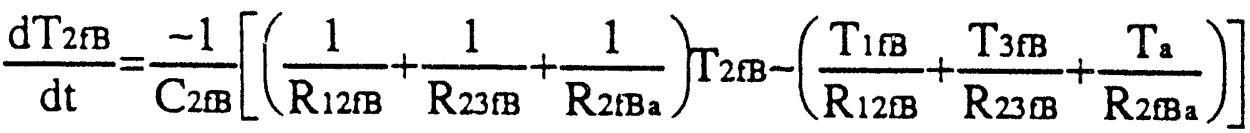

$$
\begin{aligned}
& \frac{\mathrm{dT}_{2 \mathrm{fC}}}{\mathrm{dt}}=\frac{-1}{\mathrm{C}_{2 \mathrm{fC}}}\left[\left(\frac{1}{\mathrm{R}_{12 \mathrm{fC}}}+\frac{1}{\mathrm{R}_{23 \mathrm{fC}}}+\frac{1}{\mathrm{R}_{2 \mathrm{fCa}}}\right) \mathrm{T}_{2 \mathrm{fC}}-\left(\frac{\mathrm{T}_{1 \mathrm{fC}}}{\mathrm{R}_{12 \mathrm{fC}}}+\frac{\mathrm{T}_{3 \mathrm{fC}}}{\mathrm{R}_{23 \mathrm{fC}}}+\frac{\mathrm{T}_{\mathrm{a}}}{\mathrm{R}_{2 \mathrm{fCa}}}\right)\right]
\end{aligned}
$$

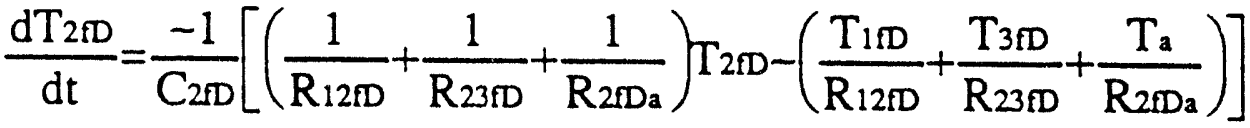

$$
\begin{aligned}
& \frac{\mathrm{dT}_{3 \mathrm{EA}}}{\mathrm{dt}}=\frac{-1}{\mathrm{C}_{3 \mathrm{FA}}}-\left[\left(\frac{1}{\mathrm{R}_{23 \mathrm{FA}}}+\frac{1}{\mathrm{R}_{\mathrm{feA}}}\right) \mathrm{T}_{2 \mathrm{fA}}-\left(\frac{\mathrm{T}_{2 \mathrm{fA}}}{\mathrm{R}_{23 \mathrm{EA}}}+\frac{\mathrm{T}_{\mathrm{e}}}{\mathrm{R}_{\mathrm{feA}}}\right)\right] \\
& \frac{d T_{3 F B}}{d t}=\frac{-1}{C_{3 E B}}\left[\left(\frac{1}{R_{23 F B}}+\frac{1}{R_{f e B}}\right) T_{2 F B}-\left(\frac{T_{2 f B}}{R_{23 A B}}+\frac{T_{e}}{R_{f A B}}\right)\right] \\
& \frac{\mathrm{dT}_{3 \mathrm{fC}}}{\mathrm{dt}}=\frac{-1}{\mathrm{C}_{3 \mathrm{fC}}}\left[\left(\frac{1}{\mathrm{R}_{23 \mathrm{fC}}}+\frac{1}{\mathrm{RfeC}_{\mathrm{f}}}\right) \mathrm{T}_{2 \mathrm{fC}}-\left(\frac{\mathrm{T}_{2 \mathrm{fC}}}{\mathrm{R}_{23 \mathrm{fC}}}+\frac{\mathrm{T}_{\mathrm{e}}}{\mathrm{RfeC}_{\mathrm{feC}}}\right)\right]
\end{aligned}
$$

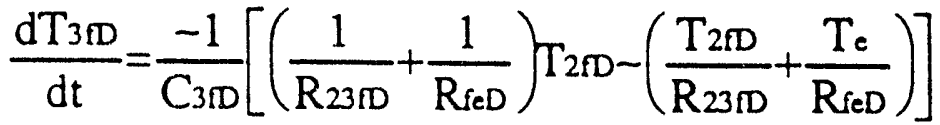

Eigure 2-7 BOWIE EIRST ELOOR SLAB NODES 


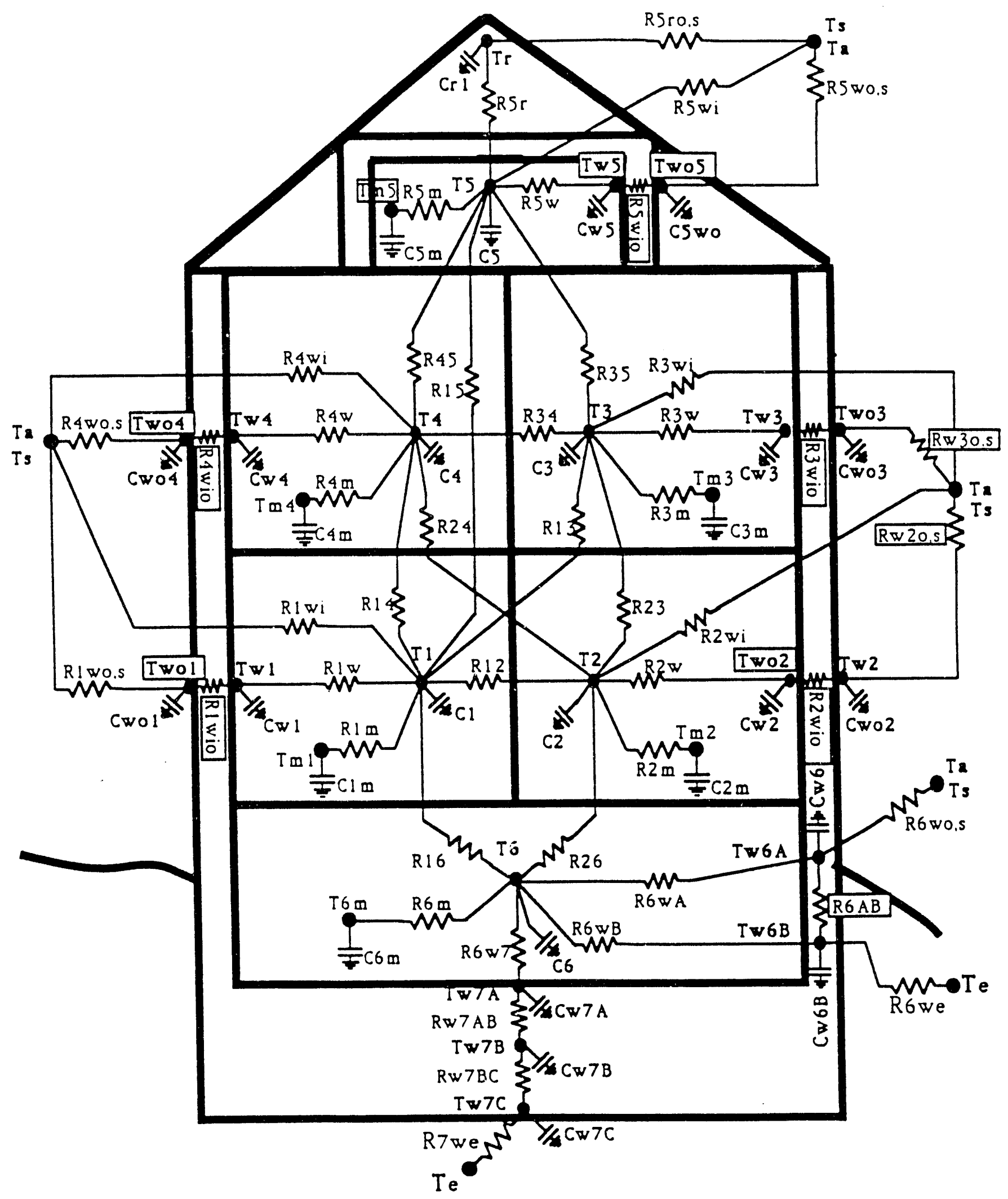

Figure 2-8 WINCHESTER THERMAL NETWORK MODEL 


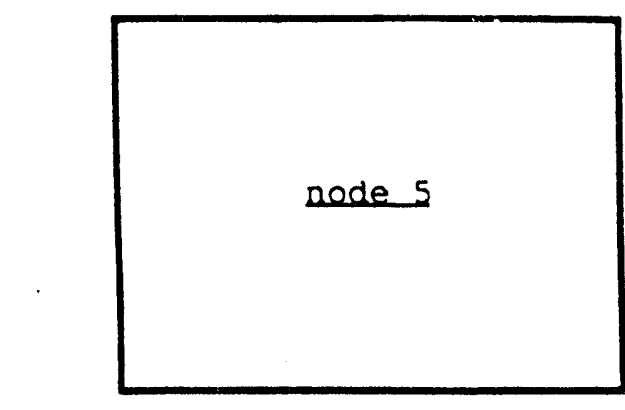

THIRD FLOOR
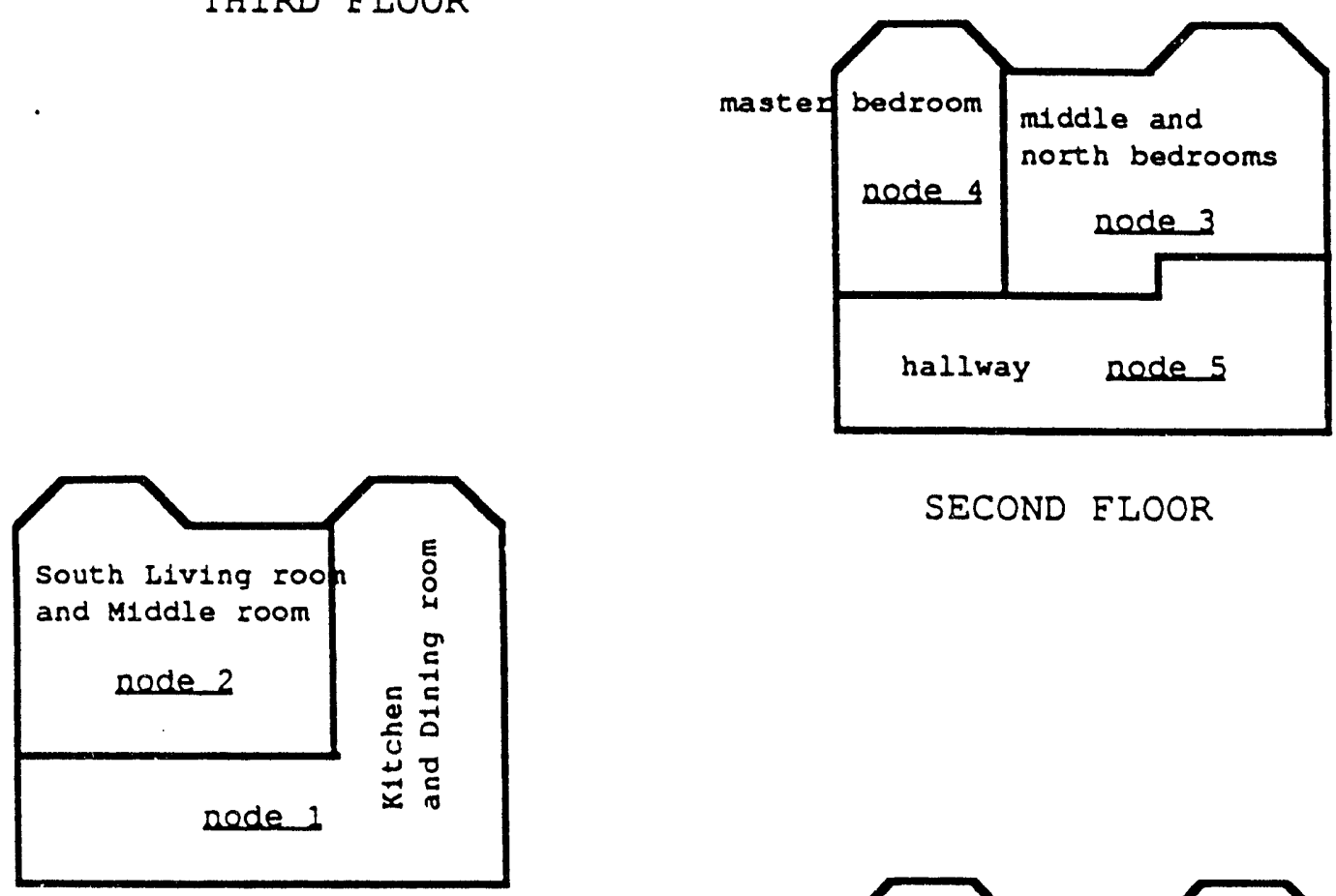

EIRST ELOOR

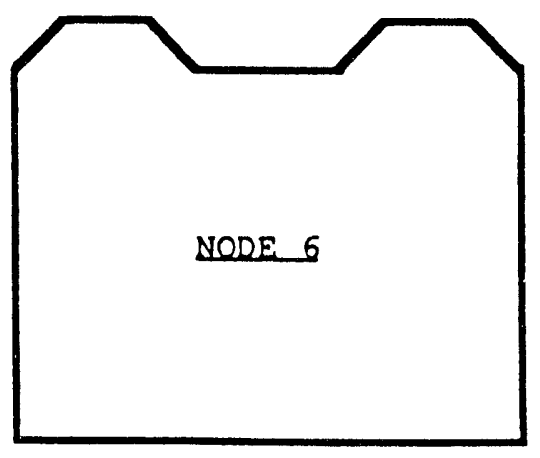

BASEMENT

Eigure 2-9 WINCHESTER NODE LOCATIONS 


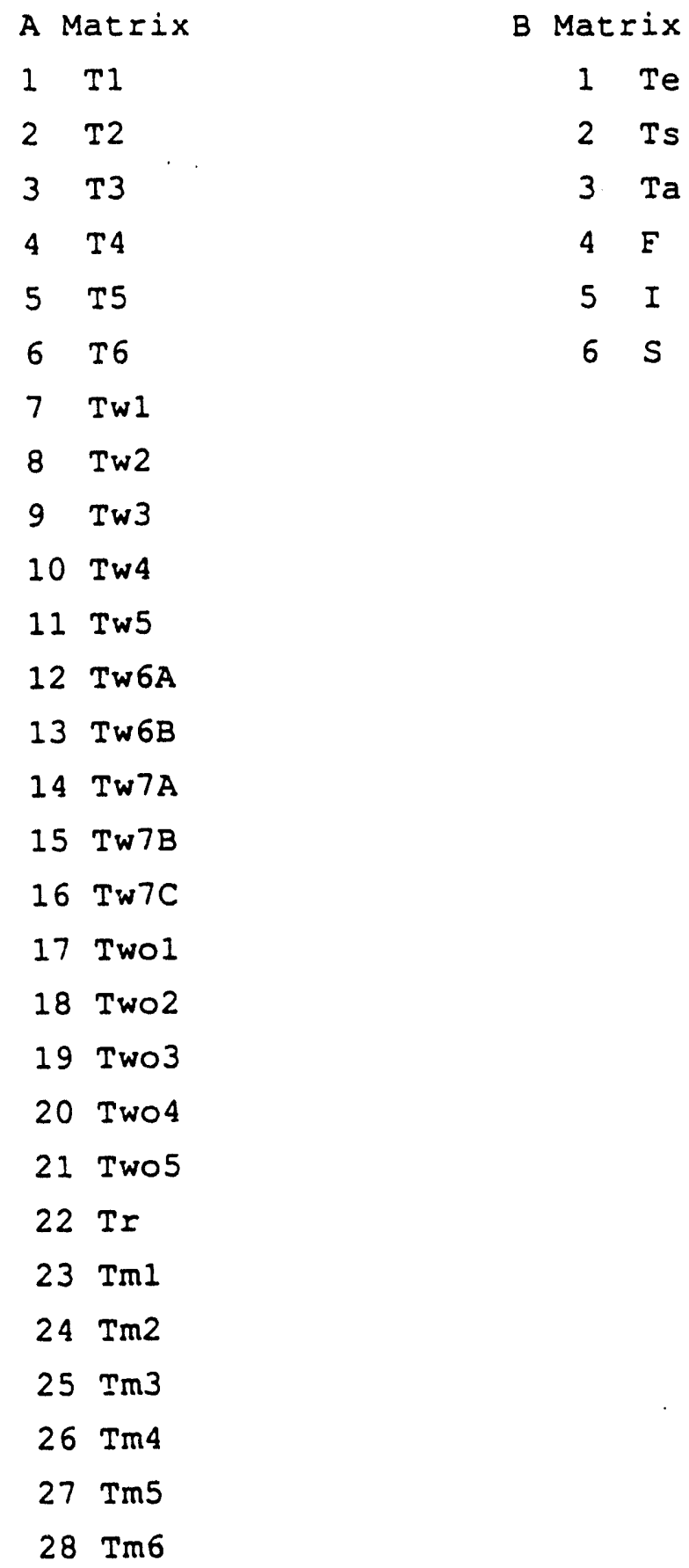

$28 \operatorname{Tm} 6$

Table 2-2 KEY TO WINCHESTER NODE ORDER 


$$
\begin{aligned}
& \frac{\mathrm{dT}_{1}}{\mathrm{dt}}=\frac{-1}{\mathrm{C}_{1}}\left[\left(\frac{1}{\mathrm{R}_{12}}+\frac{1}{\mathrm{R}_{13}}+\frac{1}{\mathrm{R}_{14}}+\frac{1}{\mathrm{R}_{15}}+\frac{1}{\mathrm{R}_{16}}+\frac{1}{\mathrm{R}_{12 \mathrm{i}}}+\frac{1}{\mathrm{R}_{14 \mathrm{i}}}+\frac{1}{\mathrm{R}_{16 \mathrm{i}}}+\frac{1}{\mathrm{R}_{1 \mathrm{a}}}+\frac{1}{\mathrm{R}_{1 \mathrm{~m}}}+\frac{1}{\mathrm{R}_{1 \mathrm{w}}}+\frac{1}{\mathrm{R}_{1 \mathrm{wi}}}\right) \mathrm{T}_{1}\right. \\
& -\left(\frac{T_{2}}{R_{12}}+\frac{T_{3}}{R_{13}}+\frac{T_{4}}{R_{14}}+\frac{T_{5}}{R_{15}}+\frac{T_{6}}{R_{16}}+\frac{T_{2}}{R_{12 i}}+\frac{T_{4}}{R_{14 i}}+\frac{T_{6}}{R_{16 i}}+\frac{T_{a}}{R_{1 a}}+\frac{T_{m 1}}{R_{1 m}}+\frac{T_{w 1}}{R_{1 w}}+\frac{T_{a}}{R_{1 w i}}\right)-\left(F_{1}-\right. \\
& \frac{\mathrm{dT}_{2}}{\mathrm{dt}}=\frac{-1}{\mathrm{C}_{2}}\left[\left(\frac{1}{\mathrm{R}_{12}}+\frac{1}{\mathrm{R}_{23}}+\frac{1}{\mathrm{R}_{24}}+\frac{1}{\mathrm{R}_{26}}+\frac{1}{\mathrm{R}_{12 \mathrm{i}}}+\frac{1}{\mathrm{R}_{23 \mathrm{i}}}+\frac{1}{\mathrm{R}_{26 \mathrm{i}}}+\frac{1}{\mathrm{R}_{2 \mathrm{a}}}+\frac{1}{\mathrm{R}_{2 \mathrm{~m}}}+\frac{1}{\mathrm{R}_{2 \mathrm{w}}}+\frac{1}{\mathrm{R}_{2 \mathrm{i}}}\right) \mathrm{T}_{2}\right. \\
& -\left(\frac{T_{1}}{R_{12}}+\frac{T_{3}}{R_{23}}+\frac{T_{4}}{R_{24}}+\frac{T_{6}}{R_{26}}+\frac{T_{2}}{R_{12 i}}+\frac{T_{3}}{R_{23 i}}+\frac{T_{6}}{R_{26 i}}+\frac{T_{a}}{R_{2 a}}+\frac{T_{m 2}}{R_{2 m}}+\frac{T_{w 2}}{R_{2 w}}+\frac{T_{a}}{R_{2 w i}}\right)-\left(F_{2}+S_{2}+I_{2}\right. \\
& \frac{\mathrm{dT}_{3}}{\mathrm{dt}}=\frac{-1}{\mathrm{C}_{3}}\left[\left(\frac{1}{\mathrm{R}_{13}}+\frac{1}{\mathrm{R}_{23}}+\frac{1}{\mathrm{R}_{34}}+\frac{1}{\mathrm{R}_{35}}+\frac{1}{\mathrm{R}_{23 i}}+\frac{1}{\mathrm{R}_{34 i}}+\frac{1}{\mathrm{R}_{35 i}}+\frac{1}{\mathrm{R}_{3 \mathrm{a}}}+\frac{1}{\mathrm{R}_{3 \mathrm{~m}}}+\frac{1}{\mathrm{R}_{3 \mathrm{w}}}+\frac{1}{\mathrm{R}_{3 \mathrm{wi}}}\right) \mathrm{T}_{3}\right. \\
& -\left(\frac{T_{1}}{R_{13}}+\frac{T_{2}}{R_{23}}+\frac{T_{4}}{R_{34}}+\frac{T_{5}}{R_{35}}+\frac{T_{2}}{R_{23 i}}+\frac{T_{4}}{R_{34 i}}+\frac{T_{5}}{R_{35 i}}+\frac{T_{a}}{R_{3 a}}+\frac{T_{m 3}}{R_{3 m}}+\frac{T_{w 3}}{R_{3 w}}+\frac{T_{2}}{R_{3 w i}}\right)-\left(F_{3}+S_{3}+I_{3}\right) \\
& \frac{\mathrm{dT}_{4}}{\mathrm{dt}}=\frac{-1}{\mathrm{C}_{4}}\left[\left(\frac{1}{\mathrm{R}_{14}}+\frac{1}{\mathrm{R}_{24}}+\frac{1}{\mathrm{R}_{34}}+\frac{1}{\mathrm{R}_{45}}+\frac{1}{\mathrm{R}_{14 i}}+\frac{1}{\mathrm{R}_{34 \mathrm{i}}}+\frac{1}{\mathrm{R}_{45 \mathrm{i}}}+\frac{1}{\mathrm{R}_{4 \mathrm{a}}}+\frac{1}{\mathrm{R}_{4 \mathrm{~m}}}+\frac{1}{\mathrm{R}_{4 \mathrm{w}}}+\frac{1}{\mathrm{R}_{4 \mathrm{wi}}}\right) \mathrm{T}_{4}\right. \\
& -\left(\frac{T_{1}}{R_{14}}+\frac{T_{2}}{R_{24}}+\frac{T_{3}}{R_{34}}+\frac{T_{5}}{R_{45}}+\frac{T_{1}}{R_{14 i}}+\frac{T_{3}}{R_{34 i}}+\frac{T_{5}}{R_{45 i}}+\frac{T_{2}}{R_{4 a}}+\frac{T_{m 4}}{R_{4 m}}+\frac{T_{w 4}}{R_{4 w}}+\frac{T_{2}}{R_{4 w i}}\right)-\left(F_{4}+S_{4}+I_{4}\right) \\
& \frac{\mathrm{dT}_{5}}{\mathrm{dt}}=\frac{-1}{\mathrm{C}_{5}}\left[\left(\frac{1}{\mathrm{R}_{15}}+\frac{1}{\mathrm{R}_{35}}+\frac{1}{\mathrm{R}_{45}}+\frac{1}{\mathrm{R}_{35 i}}+\frac{1}{\mathrm{R}_{45 i}}+\frac{1}{\mathrm{R}_{5 \mathrm{r}}}+\frac{1}{\mathrm{R}_{5 \mathrm{a}}}+\frac{1}{\mathrm{R}_{5 \mathrm{~m}}}+\frac{1}{\mathrm{R}_{5 \mathrm{w}}}+\frac{1}{\mathrm{R}_{5 \mathrm{wi}}}\right) \mathrm{T}_{5}\right. \\
& \left.-\left(\frac{T_{1}}{R_{15}}+\frac{T_{3}}{R_{35}}+\frac{T_{4}}{R_{45}}+\frac{T_{3}}{R_{35 i}}+\frac{T_{4}}{R_{45 i}}+\frac{T_{r}}{R_{5 r}}+\frac{T_{a}}{R_{5 a}}+\frac{T_{m 5}}{R_{5 m}}+\frac{T_{w 5}}{R_{5 w}}+\frac{T_{a}}{R_{5 w i}}\right)-\left(F_{5}+S_{5}+I_{5}\right)\right] \\
& \frac{\mathrm{dT}_{6}}{\mathrm{dt}}=\frac{-1}{\mathrm{C}_{6}}\left[\left(\frac{1}{\mathrm{R}_{16}}+\frac{1}{\mathrm{R}_{26}}+\frac{1}{\mathrm{R}_{16 \mathrm{i}}}+\frac{1}{\mathrm{R}_{26 \mathrm{i}}}+\frac{1}{\mathrm{R}_{6 \mathrm{a}}}+\frac{1}{\mathrm{R}_{5 \mathrm{~m}}}+\frac{1}{\mathrm{R}_{6 \mathrm{wA}}}+\frac{1}{\mathrm{R}_{6 \mathrm{wB}}}+\frac{1}{\mathrm{R}_{6 \mathrm{w} 7}}\right) \mathrm{T}_{6}\right. \\
& \left.-\left(\frac{T_{1}}{R_{16}}+\frac{T_{2}}{R_{26}}+\frac{T_{1}}{R_{16 i}}+\frac{T_{2}}{R_{26 i}}+\frac{T_{a}}{R_{6 a}}+\frac{T_{m 6}}{R_{6 m}}+\frac{T_{w 6 A}}{R_{6 w A}}+\frac{T_{w 6 B}}{R_{6 w B}}+\frac{T_{w 7}}{R_{6 w 7}}\right)-\left(F_{6}+I_{6}\right)\right]
\end{aligned}
$$

Figure 2-10 WINCHESTER AIR MASS NODES 


$$
\begin{aligned}
& \frac{d T_{w 1}}{d t}=\frac{-1}{C_{w 1}}\left[\left(\frac{1}{R_{1 w}}+\frac{1}{R_{1 w i o}}\right) T_{w 1}-\left(\frac{T_{1}}{R_{1 w}}+\frac{T_{w 01}}{R_{1 w 10}}\right)\right] \\
& \frac{d T_{w 2}}{d t}=\frac{-1}{C_{w 2}}\left[\left(\frac{1}{R_{2 w}}+\frac{1}{R_{2 w i o}}\right) T_{w 2}-\left(\frac{T_{2}}{R_{2 w}}+\frac{T_{w 02}}{R_{2 w i o}}\right)\right] \\
& \frac{d T_{w 3}}{d t}=\frac{-1}{C_{w 3}}\left[\left(\frac{1}{R_{3 w}}+\frac{1}{R_{3 w i o}}\right) T_{w 3}-\left(\frac{T_{3}}{R_{3 w}}+\frac{T_{w 03}}{R_{3 w i o}}\right)\right] \\
& \frac{d T_{w 4}}{d t}=\frac{-1}{C_{w 4}}\left[\left(\frac{1}{R_{4 w}}+\frac{1}{R_{4 w i o}}\right) T_{w 4}-\left(\frac{T_{4}}{R_{4 w}}+\frac{T_{w 04}}{R_{4 w i o}}\right)\right] \\
& \frac{d T_{w 5}}{d t}=\frac{-1}{C_{w 5}}\left[\left(\frac{1}{R_{5 w}}+\frac{1}{R_{5 w i o}}\right) T_{w 5}-\left(\frac{T_{5}}{R_{5 w}}+\frac{T_{w 05}}{R_{5 w i o}}\right)\right] \\
& \frac{d T_{w 6 A}}{d t}=\frac{-1}{C_{w 6 A}}\left[\left(\frac{1}{R_{6 w A}}+\frac{1}{R_{6 w A}}\right) T_{w 6 A}-\left(\frac{T_{6 A}}{R_{6 w A}}+\frac{T_{e}}{R_{6 w A}}\right)\right] \\
& \frac{d T_{w 6 B}}{d t}=\frac{-1}{C_{w 6 B}}\left[\left(\frac{1}{R_{6 w B}}+\frac{1}{R_{6 w e}}\right) T_{w 6 B}-\left(\frac{T_{6 B}}{R_{6 w B}}+\frac{T_{e}}{R_{6 w e}}\right)\right] \\
& \frac{d T_{w 7 A}}{d t}=\frac{-1}{C_{w 7 A}}\left[\left(\frac{1}{R_{6 w 7}}+\frac{1}{R_{w 7 A B}}\right) T_{w 7 A}-\left(\frac{T_{6}}{R_{6 w 7}}+\frac{T_{w 7 B}}{R_{w 7 A B}}\right)\right] \\
& \frac{d T_{w 7 B}}{d t}=\frac{-1}{C_{w 7 B}}\left[\left(\frac{1}{R_{w A B}}+\frac{1}{R_{w B C}}\right) T_{w 7 B}-\left(\frac{T_{w 7 A}}{R_{w A B}}+\frac{T_{w 7 C}}{R_{w B C}}\right)\right] \\
& \frac{d T_{w 7 C}}{d t}=\frac{-1}{C_{w 7 C}}\left[\left(\frac{1}{R_{w 7 B C}}+\frac{1}{R_{7 w e}}\right) T_{w 7 C}-\left(\frac{T_{w 7 B}}{R_{w 7 B C}}+\frac{T_{e}}{R_{7 w e}}\right)\right]
\end{aligned}
$$

Figure 2-11 WINCHESTER INNER WALL NODES 


$$
\begin{aligned}
& \frac{\mathrm{dT}_{\text {wol }}}{\mathrm{dt}}=\frac{-1}{\mathrm{C}_{\text {wol }}}\left[\left(\frac{1}{\mathrm{R}_{1 \text { wio }}}+\frac{1}{\mathrm{R}_{1 w_{a}}}+\frac{1}{\mathrm{R}_{1 w_{s}}}\right) \Gamma_{\text {wol }}-\left(\frac{\mathrm{T}_{1}}{\mathrm{R}_{1 \text { wio }}}+\frac{\mathrm{T}_{\mathrm{a}}}{\mathrm{R}_{1 w_{\mathrm{a}}}}+\frac{\mathrm{T}_{\mathrm{s}}}{\mathrm{R}_{1 w_{s}}}\right)-\left(\mathrm{S}_{w 1}\right)\right] \\
& \frac{\mathrm{dT}_{\text {wo2 }}}{\mathrm{dt}}=\frac{-1}{\mathrm{C}_{w 02}}\left[\left(\frac{1}{\mathrm{R}_{2 w i o}}+\frac{1}{\mathrm{R}_{2 w a}}+\frac{1}{\mathrm{R}_{2 w s}}\right) \mathrm{T}_{w 02}-\left(\frac{\mathrm{T}_{w 2}}{\mathrm{R}_{2 w i o}}+\frac{\mathrm{T}_{\mathrm{a}}}{\mathrm{R}_{2 w a}} \frac{\mathrm{T}_{s}}{\mathrm{R}_{2 w \mathrm{w}}}\right)-\left(\mathrm{S}_{w 2}\right)\right] \\
& \frac{\mathrm{dT}_{\text {wo3 }}}{\mathrm{dt}}=\frac{-1}{\mathrm{C}_{\text {w03 }}}\left[\left(\frac{1}{\mathrm{R}_{3 w i o}}+\frac{1}{\mathrm{R}_{3 w a}}+\frac{1}{\mathrm{R}_{3 w_{s}}}\right) \mathrm{T}_{w 03}-\left(\frac{\mathrm{T}_{w 3}}{\mathrm{R}_{3 w i o}}+\frac{\mathrm{T}_{\mathrm{a}}}{\mathrm{R}_{3 w a}}+\frac{\mathrm{T}_{s}}{\mathrm{R}_{3 w s}}\right)-\left(\mathrm{S}_{w 3}\right)\right] \\
& \frac{\mathrm{dT}_{\text {wo4 }}}{\mathrm{dt}}=\frac{-1}{\mathrm{C}_{\text {wo4 }}}\left[\left(\frac{1}{\mathrm{R}_{4 w_{i 0}}}+\frac{1}{\mathrm{R}_{4 w_{a}}}+\frac{1}{R_{4 w s}}\right) \mathrm{T}_{\text {wo4 }}-\left(\frac{\mathrm{T}_{w 4}}{\mathrm{R}_{4 w_{i 0}}}+\frac{\mathrm{T}_{\mathrm{a}}}{\mathrm{R}_{4 w_{a}}}+\frac{\mathrm{T}_{s}}{\mathrm{R}_{4 w 5}}\right)-\left(\mathrm{S}_{w 4}\right)\right] \\
& \frac{\mathrm{dT}_{\text {wo5 }}}{\mathrm{dt}}=\frac{-1}{\mathrm{C}_{\text {wo5 }}}\left[\left(\frac{1}{\mathrm{R}_{5 w i o}}+\frac{1}{\mathrm{R}_{5 w a}}+\frac{1}{\mathrm{R}_{5 w s}}\right) \mathrm{T}_{\text {wos }}-\left(\frac{\mathrm{T}_{w 5}}{\mathrm{R}_{5 w i o}}+\frac{\mathrm{T}_{\mathrm{a}}}{\mathrm{R}_{5 w a}}+\frac{\mathrm{T}_{s}}{\mathrm{R}_{5 w s}}\right)-\left(\mathrm{S}_{w 5}\right)\right] \\
& \frac{\mathrm{dT}_{\mathrm{r}}}{\mathrm{dt}}=\frac{-1}{\mathrm{C}_{\mathrm{r}}}\left[\left(\frac{1}{\mathrm{R}_{\mathrm{r}}}+\frac{1}{\mathrm{R}_{5_{\mathrm{ra}}}}+\frac{1}{\mathrm{R}_{\mathrm{rs}}}\right) \mathrm{T}_{\mathrm{r}}-\left(\frac{\mathrm{T}_{5}}{\mathrm{R}_{\mathrm{r}}}+\frac{\mathrm{T}_{\mathrm{a}}}{\mathrm{R}_{\mathrm{ra}}}+\frac{\mathrm{T}_{\mathrm{r}}}{\mathrm{R}_{\mathrm{rs}}}\right)-\left(\mathrm{S}_{\mathrm{r}}\right)\right]
\end{aligned}
$$

Figure 2-12 WINCHESTER OUTER WALL NODES

$$
\begin{aligned}
& \frac{\mathrm{dT}_{\mathrm{m} 1}}{\mathrm{dt}}=\frac{-1}{\mathrm{C}_{\mathrm{m} 1}}\left[\left(\frac{1}{\mathrm{R}_{1 \mathrm{~m}}}\right) \mathrm{T}_{\mathrm{m} \mathrm{1}}-\left(\frac{\mathrm{T}_{1}}{\mathrm{R}_{1 \mathrm{~m}}}\right)\right] \\
& \frac{\mathrm{dT}_{\mathrm{m} 2}}{\mathrm{dt}}=\frac{-1}{\mathrm{C}_{\mathrm{m} 2}}\left[\left(\frac{1}{\mathrm{R}_{2 \mathrm{~m}}}\right) \mathrm{T}_{\mathrm{m} 2}-\left(\frac{\mathrm{T}_{2}}{\mathrm{R}_{2 \mathrm{~m}}}\right)\right] \\
& \frac{\mathrm{dT}_{\mathrm{m} 3}}{\mathrm{dt}}=\frac{-1}{\mathrm{C}_{\mathrm{m} 3}}\left[\left(\frac{1}{\mathrm{R}_{3 \mathrm{~m}}}\right) \mathrm{T}_{\mathrm{m} 3}-\left(\frac{\mathrm{T}_{3}}{\mathrm{R}_{3 \mathrm{~m}}}\right)\right] \\
& \frac{\mathrm{dT}_{\mathrm{m} 4}}{\mathrm{dt}}=\frac{-1}{\mathrm{C}_{\mathrm{m} 4}}\left[\left(\frac{1}{\mathrm{R}_{4 \mathrm{~m}}}\right) \mathrm{T}_{\mathrm{m} 4} \sim\left(\frac{\mathrm{T}_{4}}{\mathrm{R}_{4 \mathrm{~m}}}\right)\right] \\
& \frac{\mathrm{dT}_{\mathrm{m} s}}{\mathrm{dt}}=\frac{-1}{\mathrm{C}_{\mathrm{m} s}}\left[\left(\frac{1}{\mathrm{Rsm}_{\mathrm{m}}}\right) \mathrm{T}_{\mathrm{m} \mathrm{s}}-\left(\frac{\mathrm{T}_{\mathrm{s}}}{\mathrm{Rs}_{\mathrm{m}}}\right)\right] \\
& \frac{\mathrm{dT}_{\mathrm{m} 6}}{\mathrm{dt}}=\frac{-1}{\mathrm{C}_{\mathrm{m} 6}}\left[\left(\frac{1}{\mathrm{R}_{6 \mathrm{~m}}}\right) \mathrm{T}_{\mathrm{m} 6}-\left(\frac{\mathrm{T}_{6}}{\mathrm{R}_{6 \mathrm{~m}}}\right)\right]
\end{aligned}
$$




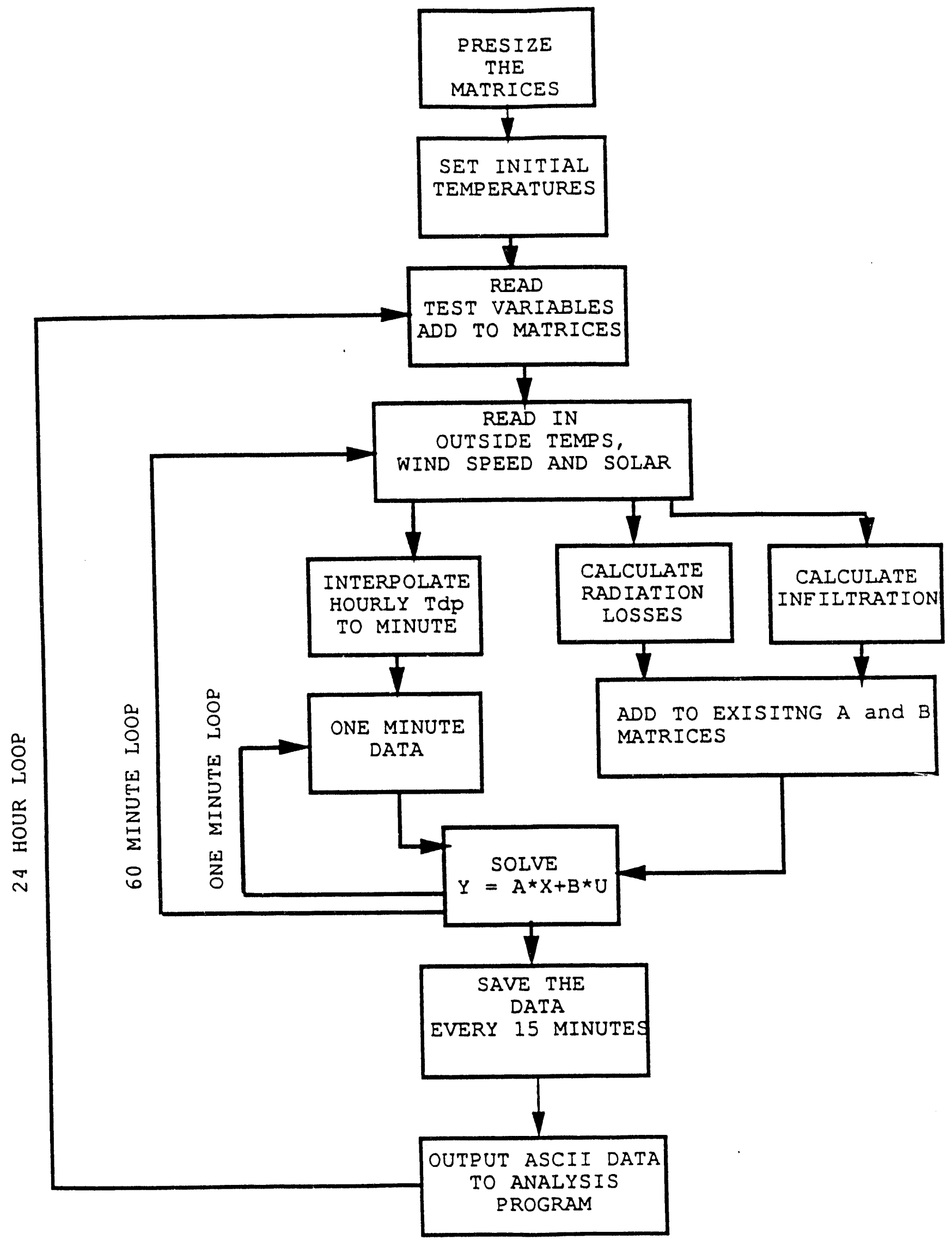

Eigure 2-14 SIMU.M PROGRAM SEQUENCE 


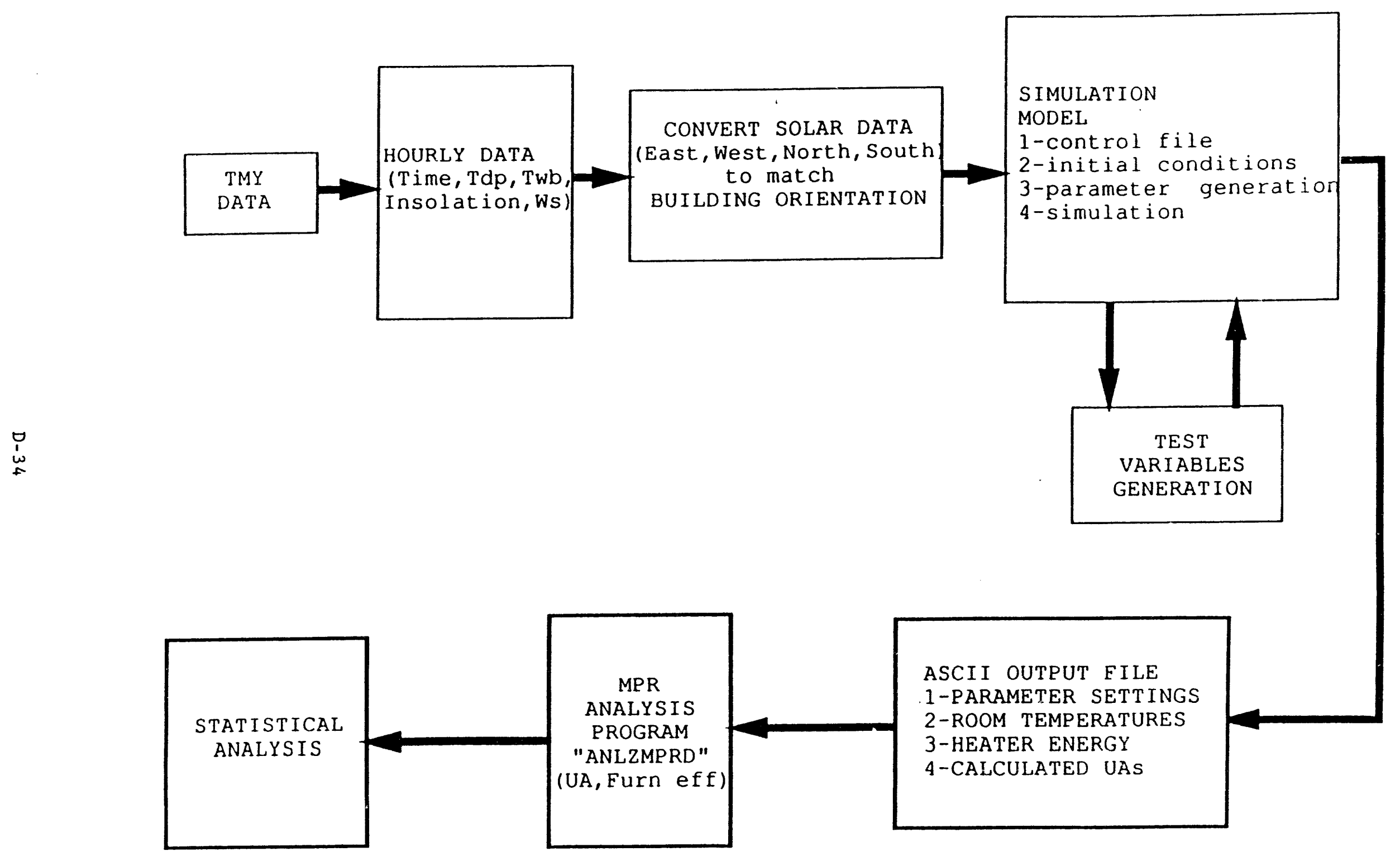

Eigure 2-15 MPR SIMULATIÚN TEST 


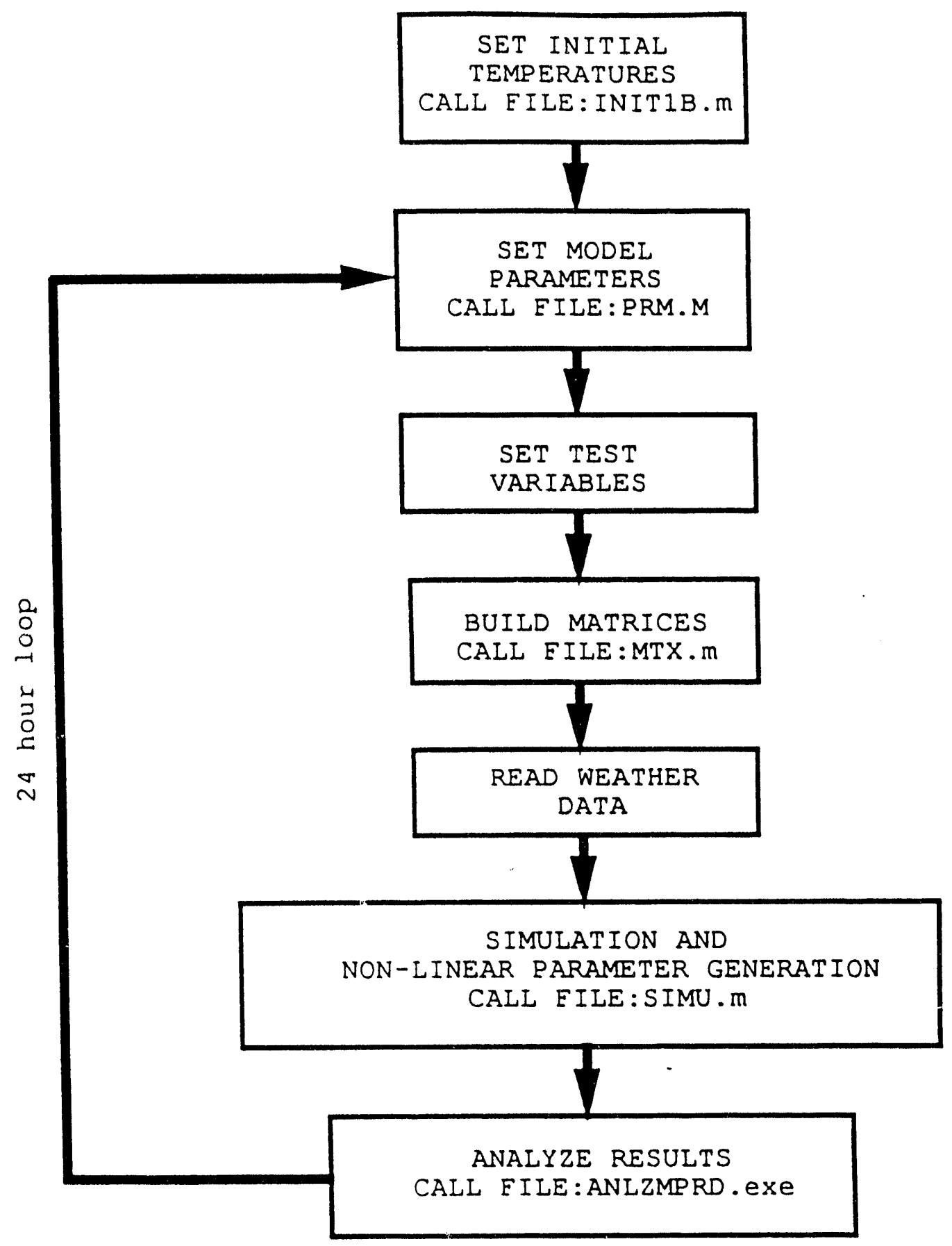

Eigure 2-16 FILE MI.m PROGRAM STEPS 


\begin{tabular}{|c|l|}
\hline COLUMN \# & DATA TYPE \\
\hline 1 & Day \\
\hline 2 & Hour \\
\hline 3 & Solar Beam Radiation \\
\hline 4 & Solar Total Horozontal \\
\hline 5 & Temperature dry bulb \\
\hline 6 & Temperature dew point \\
\hline 7 & Wind Speed \\
\hline 8 & Insolation North vertical surface \\
\hline 9 & Insolation South vertical surface \\
\hline 10 & Insolation East vertical surface \\
\hline 11 & Insolation West vertical surface \\
\hline 12 & Insolation East horozontal surface \\
\hline 13 & Insolation West horozontal surface \\
\hline 14 & Temperature Ground \\
\hline 15 & Test Number \\
\hline
\end{tabular}

Table 2-3 KEY TO WEATHER EILE 


\begin{tabular}{|c|c|c|}
\hline COLUMN \# & KOW 1 & ROWS $2: 97$ \\
\hline 1 & UAcond (BTU/HR-deaE) & dav \\
\hline 2 & UArad (BTU/HR-degE) & hour-minute \\
\hline 3 & UAinf (BTU/HR-degE) & Test \# \\
\hline 4 & UAs lab-earth (BTU/HR-ded) & Tnode 2 (deaE) \\
\hline 5 & UAs lab-ambient (BTU/HR-d) & Tnode 1 (deaE) \\
\hline 6 & UAtotEurn (BTU/HR - deaE) & Tnode I (degF) \\
\hline 7 & INE Eurnace (ACH) & Tnode l (deaE) \\
\hline 8 & INF coheat (ACH) & Inode 3 (deaE) \\
\hline 9 & Ws avg (ft/sec) & Tnode $3($ degE) \\
\hline 10 & Ws std(ft/sec) & Tnode 2 (degE) \\
\hline 11 & Tdrift (degF) & not used \\
\hline 12 & Istd (deaF) & $\mathrm{Ta}($ deg $E)$ \\
\hline 13 & Tmean (degF) & $\mathrm{Ta}($ dea $\mathrm{F})$ \\
\hline 14 & not used & Tnode 4 (degE) \\
\hline 15 & Egt & not used \\
\hline 16 & Etfm & Tnode 5 (degE) \\
\hline 17 & Ei & Tearth (degE) \\
\hline 18 & ECWO & not used \\
\hline 19 & Erad & not used \\
\hline 20 & dist (1) & not used \\
\hline 21 & diste (2) & not used \\
\hline 22 & diste (3) & not used \\
\hline 23 & diste (4) & not used \\
\hline 24 & diste (5) & not used \\
\hline 25 & Frs & not used \\
\hline 26 & $\operatorname{distf}(1)$ & not used \\
\hline 27 & $\operatorname{distf}(2)$ & not used \\
\hline 28 & distf $(3)$ & Eurn on time (min) \\
\hline 29 & $\operatorname{distf}(4)$ & Heater node 2 on time (min) \\
\hline 30 & distf (5) & Heater node 1 on time (min) \\
\hline 31 & not used & Heater node 1 on time (min) \\
\hline 32 & Eiz & Heater node 1 on time (min) \\
\hline 33 & Esq & Heater node 3 on time (min) \\
\hline 34 & Eslab & Heater node 3 on time (min) \\
\hline 35 & Etw & Heater node 2 on time (min) \\
\hline 36 & Etm & not used \\
\hline 37 & Lch & not used \\
\hline 38 & Lf & Inot used \\
\hline 39 & Cumlative Solar & not used \\
\hline
\end{tabular}

Eigure 2-4 KEY TO BOWIE MPR. AVG OUTPUT EILE 


\begin{tabular}{|c|c|c|}
\hline COLUMN \# & \begin{tabular}{r|r|} 
ROW 1 \\
\end{tabular} & ROWS 2:97 \\
\hline 1 & UAcond (BTU/HR-degF) & dav \\
\hline 2 & UArad (BTU/HR-deaE) & hour \\
\hline 3 & UAinf (BTU/HR-degE) & minute \\
\hline 4 & UAbas (BTU/HR-deaE) & Test \# \\
\hline 5 & UAearth (BTU/HR-degE & Tnode 4 (degE) \\
\hline 6 & UAtotEurn (BTU/HR-de & Tnode 3 (deaE) \\
\hline 7 & INE furnace (ACH) & Tnode 3 (degE) \\
\hline 8 & INE coheat (ACH) & Tnode 1 (degE) \\
\hline 9 & Ws avg (ft/sec) & Tnode 2 (deaE) \\
\hline 10 & Ws std $(\mathrm{ft} / \mathrm{sec})$ & Tnode 2 (deaE) \\
\hline 11 & Tdrift (degE) & Tnode6 (degE) \\
\hline 12 & Tstd (degE) & Tnode 6 (degE) \\
\hline 13 & Tmean (dea $F$ ) & $\mathrm{Ta}(\operatorname{deg} F)$ \\
\hline 14 & $\mathrm{Eq}$ & not used \\
\hline 15 & Eqt & $\mathrm{Ta}(\operatorname{deg} E)$ \\
\hline 16 & $F b$ & not used \\
\hline 17 & $E i$ & Tearth (degF) \\
\hline 18 & Ecwo & not used \\
\hline 19 & Frad & Tnode 5 (degE) \\
\hline 20 & diste (1) & Tnode 5 (degE) \\
\hline 21 & diste (2) & not used \\
\hline 22 & diste (3) & not used \\
\hline 23 & diste (4) & not used \\
\hline 24 & diste $(5)$ & not used \\
\hline 25 & diste $(6)$ & not used \\
\hline 26 & distf(1) & not used \\
\hline 27 & distf $(2)$ & not used \\
\hline 28 & $\operatorname{distf(3)}$ & Wind Speed (ft/sec) \\
\hline 29 & distf (4) & Eurn on time (min) \\
\hline 30 & $\operatorname{distf}(5)$ & Heater node 4 on time (min) \\
\hline 31 & distf $(6)$ & Heater node 3 on time (min) \\
\hline 32 & Fiz & Heater node 3 on time (min) \\
\hline 33 & Esq & Heater node 2 on time (min) \\
\hline 34 & Eslab & Heater node 2 on time (min) \\
\hline 35 & Etw & Heater node 1 on time (min) \\
\hline 36 & Etm & Heater node 6 on time (min) \\
\hline 37 & $\mathrm{Lch}$ & Heater node 6 on time (min) \\
\hline 38 & LE & not used \\
\hline 39 & Cumlative Solar & not used \\
\hline
\end{tabular}

Eigure 2-15 KEY TO WINCHESTER MPR. AVG OUTPUT EILE 


\section{SECTION 2 REEERENCES}

Kao, Y.K., 1983, Low-Voltage Beom Thermostat Performance , NBS Building Science Series 150, National Bureau of Standards

1989 ASHBAE Handbook of Eundementals, American Society of Heating, Refrigerating and Air Conditioning Engineers, Inc., At lanta, GA

Duffie, JA, Beckman, WA, 1980, SOLAR ENGINEERING OF THERMAL PROCESSES

Krieth, F, Kreider, JE, 1978, RRINCIRIES OE SOLAR ENGINEERING

Anderson, E.E., 1983, EUNDAMENTALS OE SOLAR ENERGY CONVEERSION

, Addison-Wesley 
Section 3

EXPERIMENTAL DESIGN FOR SIMULATION TESTING OF MPR TESTS

In order to test the MPR method for sensitivity to a variety of weather and house thermal parameters, the models described in the previous section were used in many repeated simulations of the MPR tests under two sets of conditions. The first set contained an entire heating season of hourly TMY data for the respective locations of each house. The MPR tests were repeated each night for 181 days of typical weather. The second set involved systematic changes of 15 parameters of thermal characteristics and lengths of MPR tests. Results corresponding to both sets of tests are presented in this section. The implications of the results to the uncertainty in the MPR method are discussed.

\subsection{A HEATING SEASON OF TESTS}

Since the winchester house is located ten miles north of Boston, typical meterological year (TMY) data from Boston was used for the winchester house simulations. The Bowie, MD, house is Iocated in the Washington, DC, area. Tom stoeffel of the National Renewable Energy Laboratory (NREL) was kind enough to send us a pre-release copy of WYEC data (TMY data with improved solar direct normal estimates) from the washington, DC, area. TMY and WYEC data has been assembled from measurements of solar irradiation and ambient temperature taken between 1952 and 1975 
(Quinlan, 1979) to represent a typical year in both monthly means and variations.

The global horizontal solar and direct beam solar irradiation, ambient dry bulb temperature, and wind speed for each hour were utilized from the TMY/WYEC data bases. The incident solar irradiation on each face of the house (north, south, east, west, and both roof halves) were estimated for each hour using wellaccepted textbook methods (e.g., Duffie and Beckman, 1991; Hsieh, 1988). The basic approach was to estimate the fractions of the irradiation that were the beam, diffuse, and reflected components. The beam component was determined by first calculating the incident angle of the beam component on each of the six surfaces and then multiplying the direct sormal hourly estimate from the data base by the cosine of each of the incident angles. The diffuse component was estimated by subtracting the beam component on the horizontal plane from the measured total horizontal irradiation and then assuming that the diffuse component is isotropic (coming uniformly from the entire sky). The diffuse component on the surface in question was then simply the horizontal diffuse component multiplied by the sky view factor (the fraction of the view of the surface open to the sky). Finally, the reflected component was estimated as the product of the total horizontal irradiation, the ground view factor, and the ground reflectance (assumed to be 0.3 ). In addition, obstacles causing shading on each surface (e.g., houses and trees) were modeled by estimating a threshold altitude angle below which the incident direct beam was set to zero.

The above calculations were performed by a program written in QuickBASIC (TM, Microsoft). The output of the houxly values were embedded in the format of statements of a MATLAB program, which was used to execute the simulation model. The listing of one of the incident solar programs (SOLWIN2.BAS for the winchester house) is contained in Appendix $C$. 
In the simulation model, the estimated absorbed hourly solar irradiation values for each of the six surfaces were obtained by multiplying the incident solar irradiation values by the estimated absorptances of exterior surfaces and by the estimated transmittance-absorptance products of windows.

The output of each simulation was in the form of fifteen-minute averages of simulated sensor readings (temperature sensors, ontimes of electric heaters, wind speed anemometers, and gas chromatograph tracer gas readings over a two-hour period during the coheat tests). This information served as the input to virtually the same analysis program used to analyze actual MPR tests (ANLZMPRD.BAS). The sequence of the MPR tests was first furnace test, then coheat test (as called for in the last MPR report: NAHB/RC, 1991). The duration of each of the two tests for both houses was six hours.

The results of the 181 days of simulations from November 1 to April 30 for each of the houses are illustrated in a series of graphs for each house. Figure 3-1 compares the predicted UA and "actual" UA. The predicted UA in each of the following graphs refers to the UA calculated by taking into account all the fifteen minute readings except the first two of the test. The heater energy output is divided by the average difference in the inside and outside temperatures. The other UA estimates obtained in the MPR analysis are discussed later. The mean predicted UA is $408 \mathrm{Btu} / \mathrm{h}-\mathrm{F}$ with the mean actual $471 \mathrm{Btu} / \mathrm{h}-\mathrm{F}$. The standard deviation is $14.4 \mathrm{Btu} / \mathrm{h}-\mathrm{F}$ ( $3 \%$ of the mean actual UA). The root mean square difference between the actual and predicted UA is 64.2. The bias is approximately $13 \%$ of the mean actual UA, while the random uncertainty is low at $3 \%$.

The relatively low random uncertainty would mean that for a weatherization "before and after" set of tests (in which any consistent bias would subtract out), a change of $7 \%$ or more would be detected at a $95 \%$ confidence level. This estimate of 
detectable UA change is based on a one-tailed z-test under the assumption that the UA estimates follow a normal distribution with known variance (John, 1990, p. 130). Thus, it appears that the MPR test has the potential of detecting relatively low levels of UA improvement.

What is some of the difference related to? Figures 3-2 and 3-3 indicate ambient temperature and infiltration to be factors. Linear regressions of the difference in actual and predicted UA versus ambient temperature and UA due to "actual" infiltration are significant with $R^{2}$ of 0.80 and 0.29 , respectively. The $R^{2}$ is the proportion of the variance of the dependent variable (UA difference) accounted for by the regression line. As ambient temperature goes up, the predicted gets closer to the actual UA. Above $50 \mathrm{~F}$, the scatter increases, outweighing the lower bias. The data seems to indicate that approximately $20 \mathrm{~F}$ should be maintained between the indoor and outdoor temperatures. The indoor temperatures could be increased for the actual MPR tests in the warmer months. In our simulations, the indoor thermostat set point was never changed $(70.25 \mathrm{~F}$ for the winchester house and 69 $F$ for the Bowie house). For infiltration, the effect is the opposite: the higher the infiltration, the more the underprediction in UA. Since the actual infiltration is simulated to be known for the middle two of the six hours of the coheat test, the cause of this increased underprediction is not immediately evident. It is clear that the infiltration component of the UA in our simulated model is the most variable component and that the infiltration should be empirically determined, preferably by tracer gas, but at least by blower door measurements and models.

Additional regressions of the difference in actual and predicted UA versus daily absorbed solar energy and ambient temperature drift indicated no significant relationships. 
The predicted furnace efficiencies average 51.1\%, while the "actual" in the model for the heating season is $46 \%$, indicating a bias of $11 \%$. The "actual" figure does not include the addition of heat from the basement to the first floor, which heat would be measured in the MPR test as heat from the furnace (and correctly so). The addition of a correction for this effect results in a change of less than $0.5 \%$ in the above values. The standard deviation of the predicted furnace efficiencies is $3.8 \%$, or about $9 \%$ of the mean. Figures $3-4$ and $3-5$ indicate the relative insensitivity of the furnace efficiency predictions to ambient temperature and to the predicted UA. It appears that we are underpredicting UA and overpredicting furnace efficiency by the same approximate percentage.

For the Bowie house, the predictions show more scatter than for the winchester house, as illustrated in Figures 3-6 through 3-9. The difference between actual and predicted UA is not significantly related to ambient temperature, temperature drift during the tests, "actual" UA due to infiltration, total UA actual, or absorbed solar energy. The mean actual UA is $1003 \mathrm{Btu} / \mathrm{h} / \mathrm{F}$, with $400 \mathrm{Btu} / \mathrm{h} / \mathrm{F}$ being contributed by the slab to air UA (no insulated slab or footings). The predicted UA average is $654 \mathrm{Btu} / \mathrm{h} / \mathrm{F}$, which represents a $35 \%$ underprediction. The rms difference between the actual and predicted UA is 402 , or $40 \%$ of the actual. The mean actual furnace efficiency is $80 \%$, while the predicted efficiency is $56 \%$, or $30 \%$ lower. The standard deviation of the predicted furnace efficiency is $22 \%$, or $28 \%$ of the actual efficiency. Again in this house, there is an underprediction of UA, but also an underprediction of furnace efficiency (instead of overprediction as in the winchester house).

There are several factors which appear to contribute to the increased bias and random differences between actual and predicted thermal characteristics of the Bowie house. The weather is warmer: during a couple of days the temperature difference between the inside and outside was very low during the tests, 
resulting in a small heater energy being divided by an even smaller temperature difference, resulting in a large UA estimate. The slab has furnace air ducts running under it with no insulation underneath or on the sides of the slab. We include slab loss to the ground and to the air (from the sides) in the total actual UA by normalizing with respect to room air and outside air temperature difference. On some occasions, the slab surface temperature and the room air temperature are nearly the same, resulting in no actual heat loss. However, the UA values are included in the "actual" total UA. The Bowie model radiation UA is higher than that of the winchester house. The models are highly dependent on the data used to calibrate them. The Bowie house had no special tests done in order to perturb the system so as to bring out the thermal parameters, since this modeling effort was not envisioned at the time the tests were performed. The winchester house did have some tests done with varying temperature drops between zones and with cooldown tests.

There are four UA predictions being examined in the MPR analysis development: (a) the simplest, formed by taking all the 15-minute data except the first two and dividing the heater energy by the average inside-outside temperature difference (b) the UA formed by selecting an optimum window of data in which the 15-minute heater energy divided by the temperature difference has a low slope compared to scatter, (c) the UA formed by selecting another window represented by the most consistent trend in the same 15-minute heater energy divided by temperature difference (i.e., where the regression line fit to the data has the slope closest to zero), and (d) the UA in (b) corrected for thermal discharge (or recharge) due to cooldown (or warmup) in the building temperature. The method of correction for (d) is given in Duffy and Saunders (1987) or NAHB/RC (1991). The issue of which of the four UA is the closest to the simulated UA was addressed by computing the mean, the standard deviation, and the root-mean-square (rms) difference in actual and predicted UA. Not much difference appears among the four methods, as can be 
noted from Table 3-1, although a paired $t$-test indicates that the fourth method is significantly different from the other three. None of the fourth methods results in a predicted UA that is not significantly different from zero (the $t$ statistics are listed in Table 3.1).

There is actually a fifth UA estimation based on correcting for the "ramp-up" of fifteen-minute values of heater energy divided by temperature difference. It was not included in the above discussion since it is 'unly used if there is significant "rampup." This approach (developed by Duffy and reported in NAHB/RC, 1991) involves curve fitting with a three parameter equation exponential in time (characteristic of air temperatures being influenced by thermal mass charging, i.e., first-order system step responses). The estimated UA becomes the estimated steady-state value of UA from the fitted equation. For those cases in the Bowie house where ramp-up occurred (more than $2 \mathrm{~F}$ shift in interior temperatures), the predicted UA was actually much closer to the actual (predicted steady-state UA mean: $1200 \mathrm{Btu} / \mathrm{h}-\mathrm{F}$; actual UA mean: $1003 \mathrm{Btu} / \mathrm{h}-\mathrm{F}$; predicted UA of type (a) mean: 654 $\mathrm{Btu} / \mathrm{h}-\mathrm{F})$. In both houses, however, the predicted steady-state UA values were higher than the actual. Winchester had a mean predicted steady-state UA of $634 \mathrm{Btu} / \mathrm{h}-\mathrm{F}$ compared an actual UA mean of $471 \mathrm{Btu} / \mathrm{h}-\mathrm{F}$. This approach may have some merit but needs some additional development.

A question naturally arises as to the cause(s) of the discrepancies between the "actual" and predicted values of UA and furnace efficiency. Is the MPR at fault or are the simulation models at fault? Without more calibration data with which to test the models, we cannot be sure of the models. As the previous discussion of the Bowie model indicates, we have some doubts about that model. As a first step in examining the sensitivity of results to calibration of even the winchester model, a modest recalibration of the winchester model was performed. The same heating season analysis was repeated. The predicted UA compared 
to the actual UA improved: the bias went from $13 \%$ to $10 \%$ of the actual and the standard deviation remained at $3 \%$. The regression of predicted UA on actual UA improved (Fig. 3-10), with the intercept going to almost zero (4) and the slope (0.88) getting closer to one, with the $r^{2}$ remaining roughly the same. Sensitivity of the difference between the predicted and actual UA to ambient mean temperature was greatly reduced $\left(r^{2}=0.00001\right)$. Consequently, the models are undoubtedly a source of some of the discrepancy between the "actual" and MPR predicted values.

In fact, the lack of methods with which to estimate accurately the "real" UA in a building, with which to compare the MPR predictions to, points out even more strongly the need for a method like the MPR with which to estimate the UA empirically.

TABLE 3-1

Comparison of UA Estimation Methods

\begin{tabular}{|l|r|r|r|r|}
\hline Winchester & UAa-UAcoh (a) & UAa-UAhyp (b) & UAa-UAtre (c) & UAa-UAccld (d) \\
\hline$t$ statistic: & 784.43 & 806.05 & 714.63 & 561.09 \\
\hline mean: & 62.59 & 63.43 & 62.41 & 56.39 \\
\hline rms difference: & 64.23 & 65.01 & 64.38 & 59.25 \\
\hline stand. deviation: & 14.44 & 14.24 & 15.81 & 18.19 \\
\hline & & & & \\
\hline Bowie & & & & 270.20 \\
\hline$t$ statistic: & 315.06 & 316.73 & 354.27 & 337.13 \\
\hline mean: & 348.79 & 348.01 & 426.41 & 419.96 \\
\hline rms difference: & 402.25 & 400.83 & 17.64 & 18.61 \\
\hline stand. deviation: & 14.89 & 14.78 & & \\
\hline
\end{tabular}




\subsection{MULTI-PARAMETER ANALYSIS}

The foregoing simulations took into account weather parameters, but what about the effect of changing the amount of thermal mass in the walls of the simulated houses? How about the effect of not coheating a room? What about changing the furnace hot air distribution pattern? What about the effect of several parameters changing at the same time?

To answer these questions, one would need to vary parameters in the simulation models in such a way that interaction of one parameter on another would be taken into account. Varying several parameters at different values each requires a high number of tests. Running $\mathrm{k}$ values of $\mathrm{m}$ parameters requires $\mathrm{k}^{\mathrm{m}}$ tests. We identified fifteen parameters to test. These are listed in Tables 3-2 and 3-3. For fifteen parameters at four values each, $4^{15}$, or 1 billion, tests would have to be performed! A great economy can be had by running just two values (two levels, in statistical terms) (e.g., Box, Hunter, Hunter, 1978). However, even $2^{15}$ is 32,000 . Our simulations take one minute on a $33 \mathrm{mHz}$ 486 PC for the winchester house and about 1.5 minutes for the Bowie house. Even the faster simulation would take 22 days to complete! 
TABLE 3-3

Bowie House Simulations

\begin{tabular}{|r|r|r|l|l|l|}
\hline & Calc: & $\begin{array}{l}\text { Diff } \\
\text { UAact- }\end{array}$ & $\begin{array}{l}\text { Diff } \\
\text { UAact- }\end{array}$ & Parameter & \\
\hline Eff.furn & UAact & UApred & UAcorcldn & & \\
\hline 0.52 & 903.15 & 469.86 & 457.40 & Lch & Coheat 4 hrs, furnace test 10 hrs \\
\hline 0.77 & 888.93 & 236.27 & 203.81 & Lf & Coheat 10 hrs, furnace test 4 hrs \\
\hline 0.82 & 898.86 & 238.65 & 213.47 & Baseline & Coheat 8 hrs, furnace test 8 hrs \\
\hline 0.82 & 900.32 & 272.19 & 218.96 & Fgt & Decrease slab loss to outside air \\
\hline 0.63 & 878.38 & 285.17 & 294.93 & Ftfm & Decrease slab mass initial temperature \\
\hline 0.75 & 837.63 & 170.80 & 158.02 & Fi & Reduce infiltration loss \\
\hline 0.51 & 897.30 & 397.00 & 397.00 & Fcwo & Reduce exterior wall mass \\
\hline 0.81 & 884.65 & 220.79 & 201.59 & Frad & Reduce radiation UA \\
\hline 1.11 & 887.72 & 407.34 & -90.55 & nocohkit & No coheat kitchen, family rm, 1st floor \\
\hline 0.52 & 899.99 & 436.12 & 399.85 & nocohbdrm & No coheat bedroom, 2nd fl \\
\hline 0.18 & 902.76 & 625.54 & 620.73 & furnlessfl & Reduce furnace air by 50\%, inc to slab \\
& & & & & $50 \%$ \\
\hline 0.48 & 901.29 & 604.99 & 668.98 & Fiz & Increase interzone heat transfer \\
\hline 0.91 & 900.84 & 343.03 & 297.41 & Fsg & Increase loss to ground \\
\hline 0.71 & 901.33 & 502.24 & 509.01 & Fslab & Decrease slab mass \\
\hline 0.54 & 899.28 & 436.33 & 414.05 & Ftw & Reduce initial temp interior wall mass \\
\hline 0.52 & 900.06 & 427.90 & 419.76 & Fim & Reduce initial temp. interior room mass \\
\hline & & & & & \{Note: Increases and decreases are by \\
\hline & Mean: & 379.64 & 336.53 & & a factor of two unless otherwise stated \\
\hline & & & &
\end{tabular}

A way out of this dilemma was tc use designs for screening experiments. We chose a design matrix for an $L(16)$ lattice for fifteen factors in 16 runs (John, 1991, p. 301). In this approach, the trade off in reducing the number of experiments is that the main effects from each factor (thermal or test parameter in our experiment) are aliased or confouncied with higher order interactions of factors. Even so this approach is good for screening in the hopes that a few facrurs will stand out and be detected (John, 1991) and is better than only changing just one parameter at a time. We did perform the latter experiment, however, also since it requires only fifteen runs. In both 
TABLE 3-2

Winchester Simulations

\begin{tabular}{|r|r|r|r|l|l|}
\hline & Calc: & $\begin{array}{l}\text { Diff } \\
\text { UAact- }\end{array}$ & $\begin{array}{l}\text { Diff } \\
\text { UAact- }\end{array}$ & Parameter & \\
\hline Eff.furn & UAact & UAcohls2 & UAcorcldn & & \\
\hline 0.48 & 472.10 & 36.99 & 38.24 & Fg & Increase ground loss from basement \\
\hline 0.47 & 462.11 & 42.54 & 43.82 & Fgt & Decrease basement loss to outside air \\
\hline 0.48 & 468.01 & 42.37 & 42.56 & Fb & Increase loss from 1st fl to basement \\
\hline 0.49 & 367.37 & 38.87 & 39.01 & Fi & Reduce infiltration loss \\
\hline 0.46 & 470.25 & 105.78 & 103.69 & Fcwo & Reduce exterior wall mass \\
\hline 0.48 & 454.81 & 38.11 & 38.82 & Frad & Reduce sky temp \\
\hline 0.43 & 465.98 & 76.03 & 77.84 & nocohsbdrm & No coheat s. bdrm \\
\hline 0.50 & 491.13 & 36.60 & 36.29 & nocohbas & No coheat basement \\
\hline 0.51 & 470.01 & 68.47 & 44.76 & furnlessfl1 & Reduce furnace to 1st floor by 35\% \\
\hline 0.63 & 482.51 & 52.59 & 53.72 & furnmorefl1 & Increase furnace to 1st floor by 20\% \\
\hline 0.47 & 471.25 & 56.48 & 58.69 & Fiz & Increase interzone heat transfer \\
\hline 0.48 & 470.06 & 44.91 & 44.88 & Lch & Inc. coheat by 2 hrs, dec. furn. by 2 hrs \\
\hline 0.50 & 470.17 & 36.98 & 35.78 & Lf & Inc. furn test by 2 hrs, dec. coheat by 2 \\
\hline 0.45 & 462.91 & 29.13 & 26.86 & Ftw & Reduce initial temp interior wall mass \\
\hline 0.46 & 462.80 & 37.90 & 38.48 & Ftm & Reduce initial temp. interior room mass \\
\hline & & & & & \\
\hline & mean: & 49.58 & 48.23 & & \{Note: Increases and decreases are by \\
\hline & & & & & a factor of two unless otherwise stated\} \\
\hline
\end{tabular}


experiments, the thermal/test parameters, or factors, had two levels: the level in the default model used in the heating season analysis, representing the high level, and a level representing a drastic change by a factor of two (in most cases), representing the low level. Tables $3-2$ and $3-3$ contain the level changes along with the results of using those changes one at a time in a simulation. The weather data was the same for each run and corresponds to January 2 for the winchester house and January 11 for the Bowie house. The exact hourly weather data is listed in programs MPIREV.M and MPIBOW.M for the winchester and Bowie houses, respectively, along with the exact parameter level changes. The winchester house ambient temperatures averaged approximately $33 \mathrm{~F}$, while the Bowie temperatures averaged $20 \mathrm{~F}$, during the night.

It is obvious from Table 3-2 that reducing the wall exterior mass has the greatest impact in the winchester house on the difference between the actual and predicted UA increasing it to $105 \mathrm{Btu} / \mathrm{h}-\mathrm{F}$ above the average of all the differences is $49 \mathrm{Btu} / \mathrm{h}-\mathrm{F}$. For the Bowie house, the mean difference between the actual and predicted UA for all the parameter level changes is $380 \mathrm{Btu} / \mathrm{h}-\mathrm{F}$. Reducing infiltration loss, reducing furnace air to the heated zone, and increasing interzone heat transfer appear to stand out as having large impacts.

The results of using the $I(16)$ lattice experimental design with the parameters are summarized in Tables 3-4 and 3-5. Regression analysis was used to estimate the effect on each parameter level change on both the difference between the actual and predicted UA and the predicted furnace efficiency tests. The factor which stands out for the winchester house in the UA estimation is again reducing the exterior wall mass. But also not putting an electric heater in the south bedroom during the coheat test affects both the UA estimate and the furnace efficiency prediction. 
For the Bowie house, reducing the furnace output to the air while increasing the output to the slab by a factor of two has a dramatic effect on both the $U A$ and furnace efficiency predictions. In fact, in at least one test involving a decreased initial slab temperature along with the increased furnace output to the slab, the furnace was on all day and increased the slab heat content enough so that the furnace and heaters essentially did not go on the entire night. Consequently, the estimated UA was zero. Obwiously, the implication for houses with in-slab heating is to monitor the initial slab conditions prior to MPR testing.

The broader implications of the simulation results are discussed in the last section.

TABLE 3-4

Winchester House Simulations with Parameter Changes According to an L(16) Lattice

\begin{tabular}{|c|c|c|c|}
\hline $\begin{array}{l}\text { UAact - UAcohls2 vs. } \\
\text { parameters }\end{array}$ & & & \\
\hline & Regression Output: & & \\
\hline Constant & & & 96.37 \\
\hline $\begin{array}{l}=\text { mean of y } \\
\text { (difference in actual } \\
\text { and predicted UA) }\end{array}$ & & & \\
\hline Std Err of Y Est & & & 0.24 \\
\hline R Squared & & & 1.00 \\
\hline No. of Observations & & & 16.00 \\
\hline Degrees of Freedom & & & 1.00 \\
\hline Parameter: & $\mathrm{X}$ Coefficient(s) & Std Err of Coef. & Estimated effect on y: \\
\hline $\mathrm{Fg}$ & -1.15 & 0.06 & -2.30 \\
\hline Fgt & 0.94 & 0.06 & 1.89 \\
\hline $\mathrm{Fb}$ & 3.38 & 0.06 & 6.76 \\
\hline
\end{tabular}




\begin{tabular}{|c|c|c|c|}
\hline $\mathrm{Fi}$ & 8.56 & 0.06 & 17.13 \\
\hline Fcwo & -28.90 & 0.06 & -57.81 \\
\hline Frad & 1.06 & 0.06 & 2.13 \\
\hline nocohsbdrm & -21.59 & 0.06 & -43.18 \\
\hline nocohbas & 0.37 & 0.06 & 0.73 \\
\hline furnlessfl1 & -6.24 & 0.06 & -12.49 \\
\hline furnmorefl1 & -3.61 & 0.06 & -7.22 \\
\hline Fiz & -3.22 & 0.06 & -6.43 \\
\hline Lch & 3.42 & 0.06 & 6.84 \\
\hline Lf & 1.84 & 0.06 & 3.68 \\
\hline Ftw & 3.79 & 0.06 & 7.58 \\
\hline & & & \\
\hline & & & \\
\hline \multicolumn{4}{|l|}{$\begin{array}{l}\text { Furnace efficiency vs } \\
\text { parameters }\end{array}$} \\
\hline & Regression Output: & & \\
\hline \multirow[t]{2}{*}{ Constant } & & & 0.50 \\
\hline & $\begin{array}{l}=\text { mean of y (furnace } \\
\text { efficiency) }\end{array}$ & & \\
\hline Std Err of Y Est & & & 0.06 \\
\hline R Squared & & & 0.89 \\
\hline No. of Observations & & & 16.00 \\
\hline Degrees of Freedom & & & 2.00 \\
\hline Parameter: & $\mathrm{X}$ Coefficient(s) & Std Err of Coef. & Estimated effect on y: \\
\hline $\mathrm{Fg}$ & 0.00 & 0.02 & 0.01 \\
\hline Fgt & 0.00 & 0.02 & 0.00 \\
\hline $\mathrm{Fb}$ & -0.01 & 0.02 & -0.01 \\
\hline $\mathrm{Fi}$ & -0.01 & 0.02 & -0.03 \\
\hline Fcwo & 0.01 & 0.02 & 0.02 \\
\hline Frad & 0.00 & 0.02 & 0.00 \\
\hline nocohsbdrm & 0.04 & 0.02 & 0.08 \\
\hline
\end{tabular}




\begin{tabular}{|l|r|r|r|}
\hline nocohbas & -0.02 & 0.02 & -0.04 \\
\hline furnlessf1 & 0.01 & 0.02 & 0.01 \\
\hline furnmorefl1 & -0.04 & 0.02 & -0.08 \\
\hline Fiz & -0.01 & 0.02 & -0.01 \\
\hline Lch & 0.00 & 0.02 & 0.00 \\
\hline Lf & 0.00 & 0.02 & 0.01 \\
\hline Ftw & 0.02 & 0.00 & 0.05 \\
\hline & & & \\
\hline
\end{tabular}

TABLE 3-5

Bowie House Simulations with Parameter Changes According to an $L(16)$ Lattice

\begin{tabular}{|l|r|r|r|}
\hline $\begin{array}{l}\text { UAact - UAcohls2 vs. } \\
\text { parameters }\end{array}$ & & & \\
\hline & Regression Output: & & \\
\hline Constant & & & 664.54 \\
\hline $\begin{array}{l}\text { = mean of y (difference } \\
\text { in actual and predicted } \\
\text { UA) }\end{array}$ & & & \\
\hline Std Err of Y Est & & & 200.84 \\
\hline R Squared & & & 0.86 \\
\hline No. of Observations & & & 16.00 \\
\hline Degrees of Freedom & & & 2.00 \\
\hline & & & \\
\hline Parameter: & Coefficient(s) & Std Err of Coef. & \\
\hline & 9.30 & & Estimated effect on y: \\
\hline Fgt & -59.83 & 50.21 & 18.59 \\
\hline Ftfm & 41.47 & 50.21 & -119.65 \\
\hline Fi & 34.48 & 50.21 & 82.93 \\
\hline Fcwo & 60.78 & 50.21 & 68.95 \\
\hline Frad & -37.41 & 50.21 & 121.56 \\
\hline nocohkit & -29.79 & 50.21 & -74.82 \\
\hline nocohbdrm & -108.59 & -59.58 \\
\hline furnlessfl1 & -45.34 & 50.21 & -217.18 \\
\hline Fiz & 36.10 & 50.21 & -90.68 \\
\hline Fsg & & 50.21 & 72.21 \\
\hline
\end{tabular}




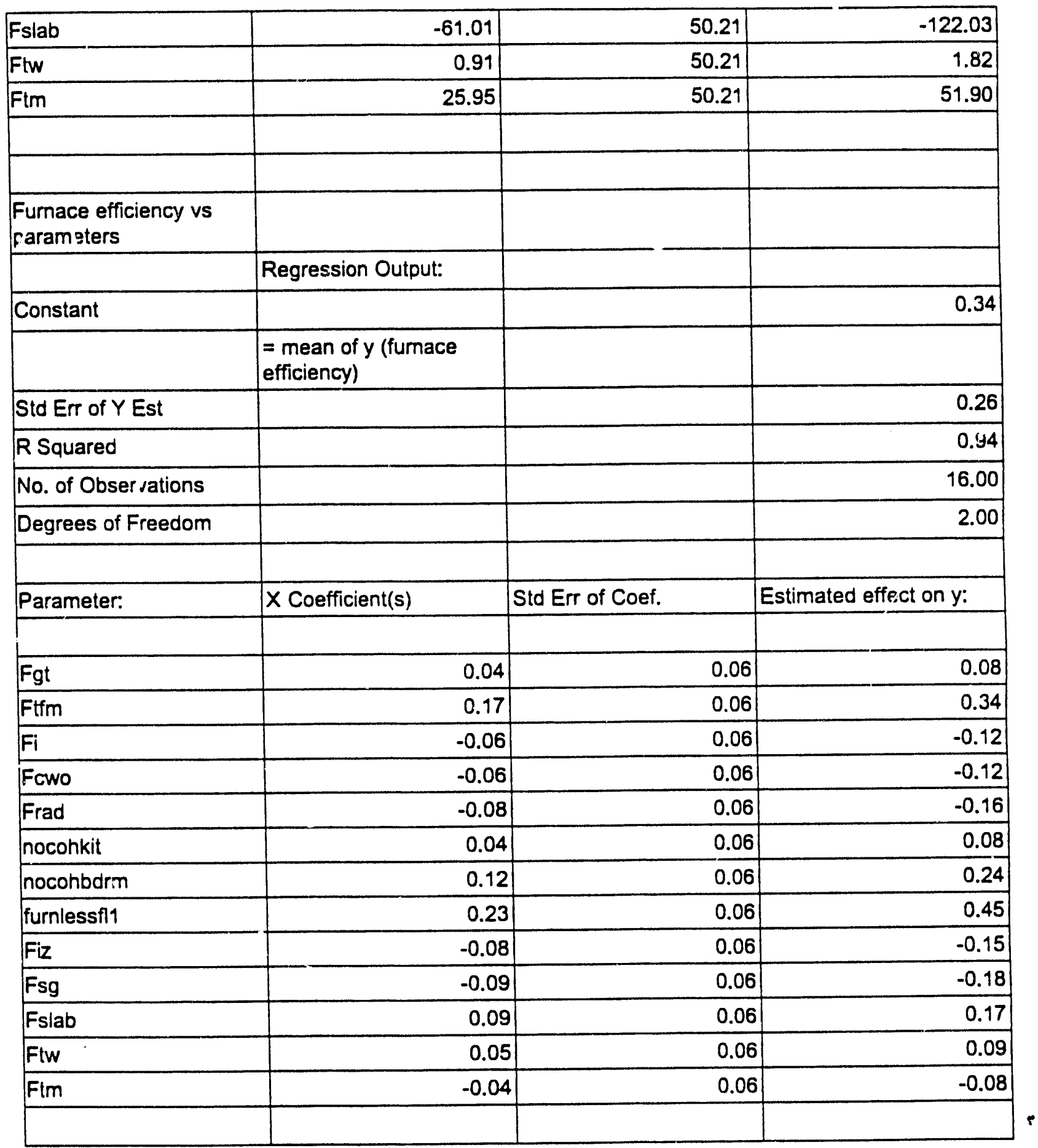




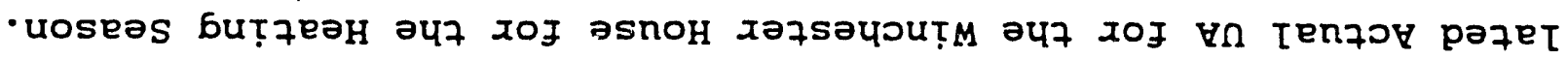

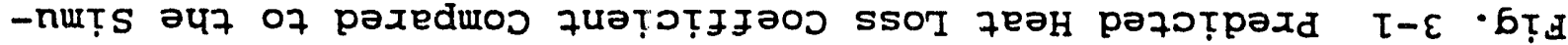

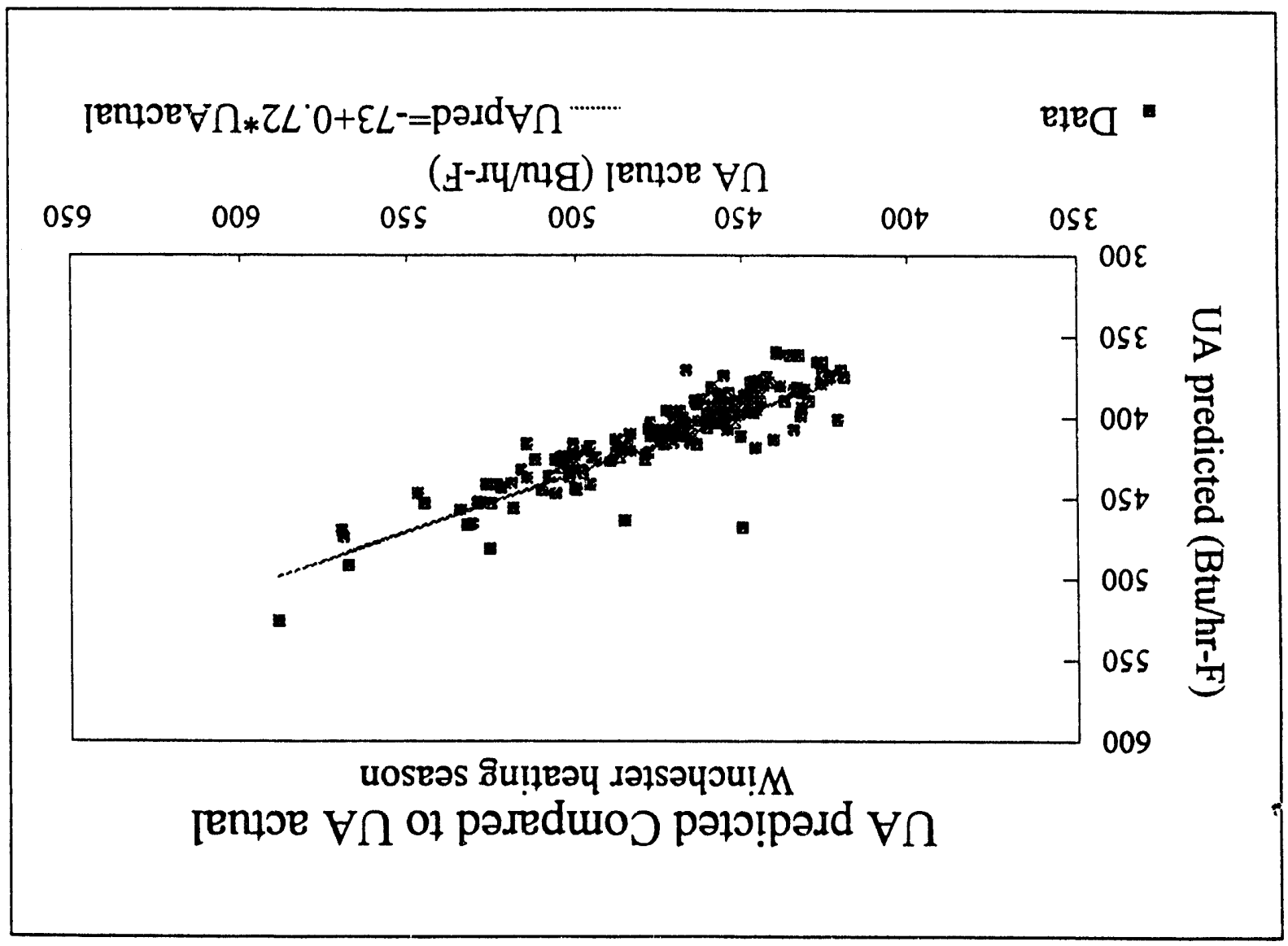




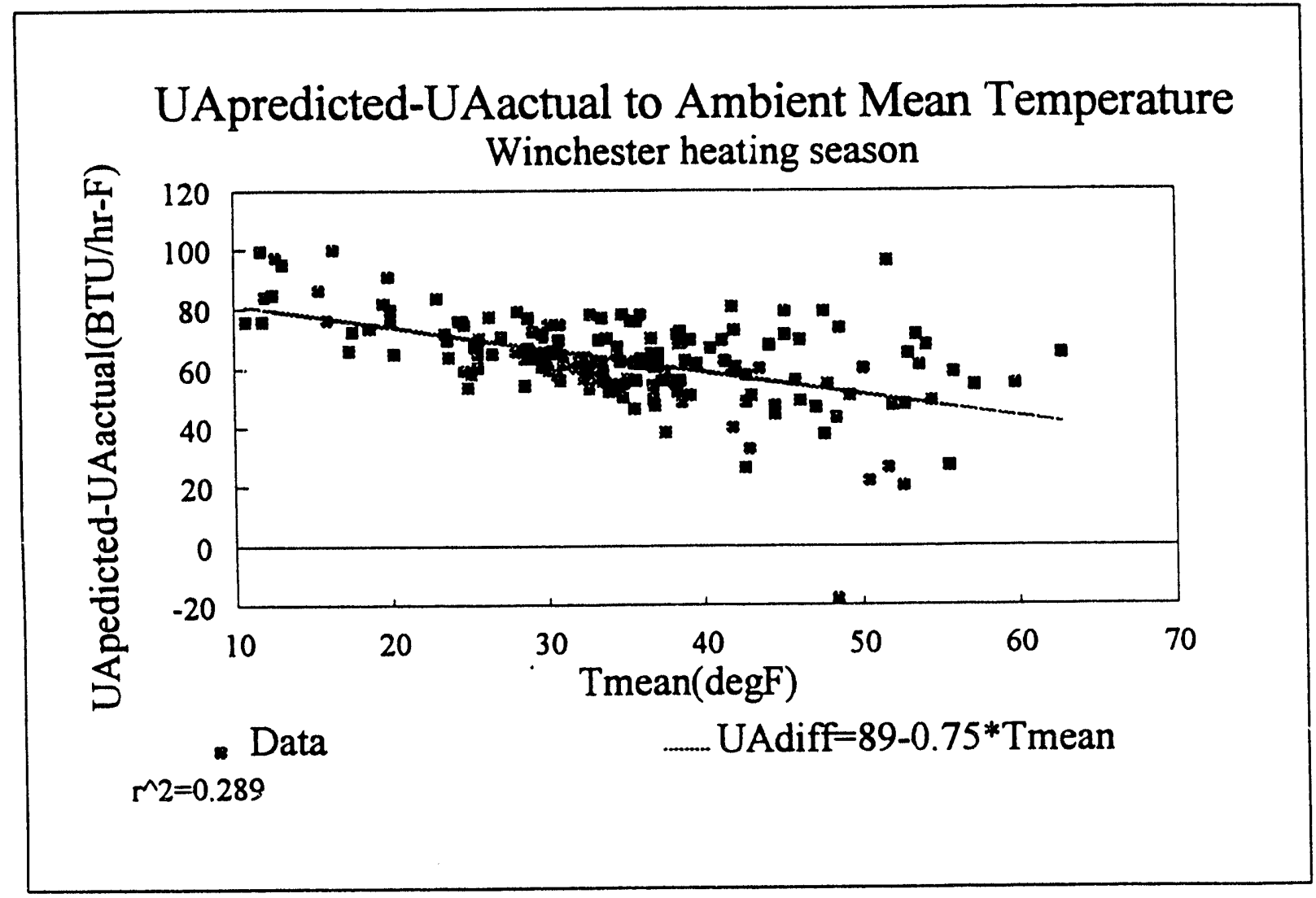

Fig. 3-2. Sensitivity of the Difference in Actual and Predicted UA to Ambient Temperature Average During the Coheat Test. 


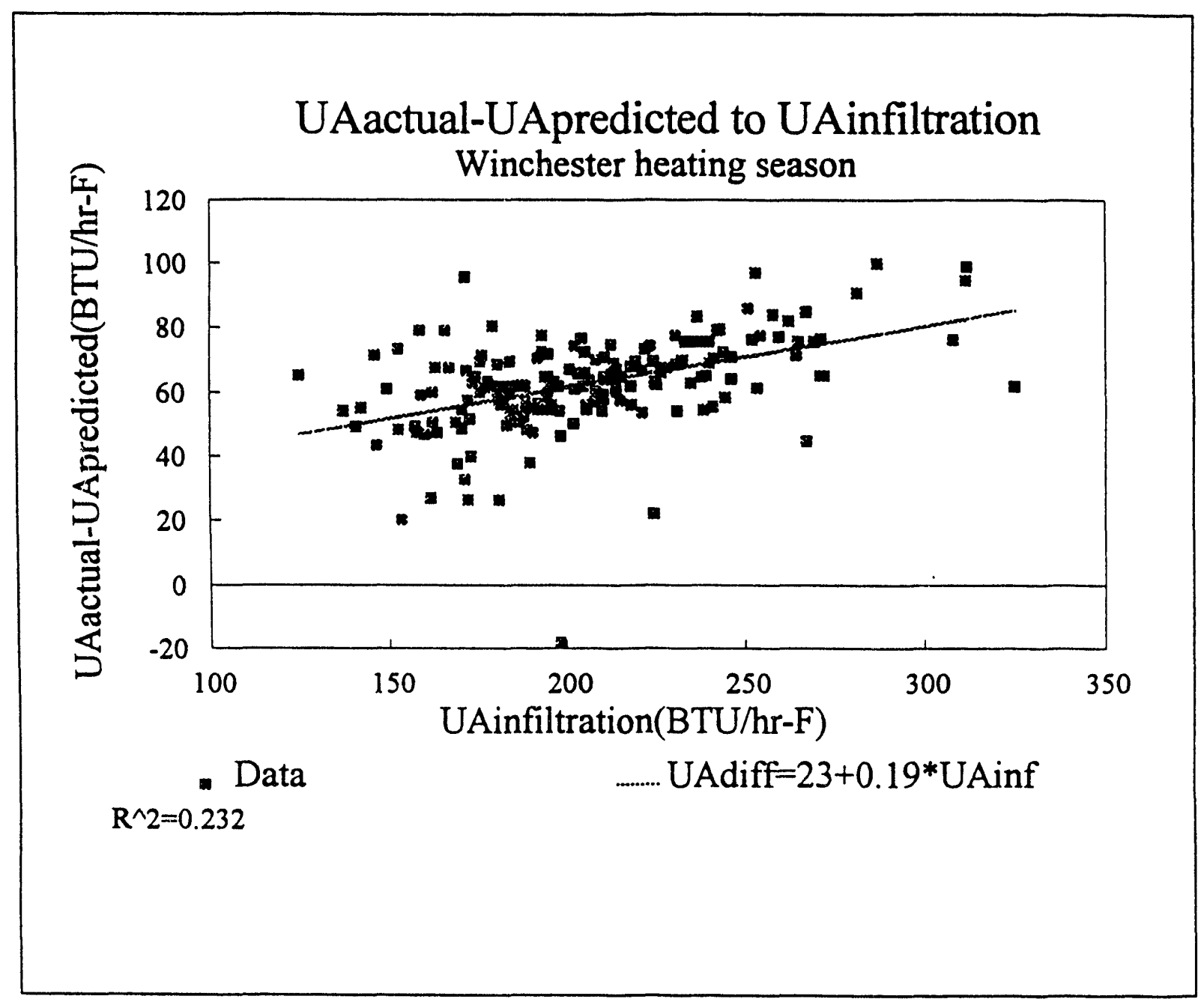

Fig. 3-3. Sensitivity of the Difference in Actual and Predicted UA to the component of Actual UA due to Infiltration. 
Predicted Furnace Efficiency Compared to Ambient Mean Temperature Winchester heating season

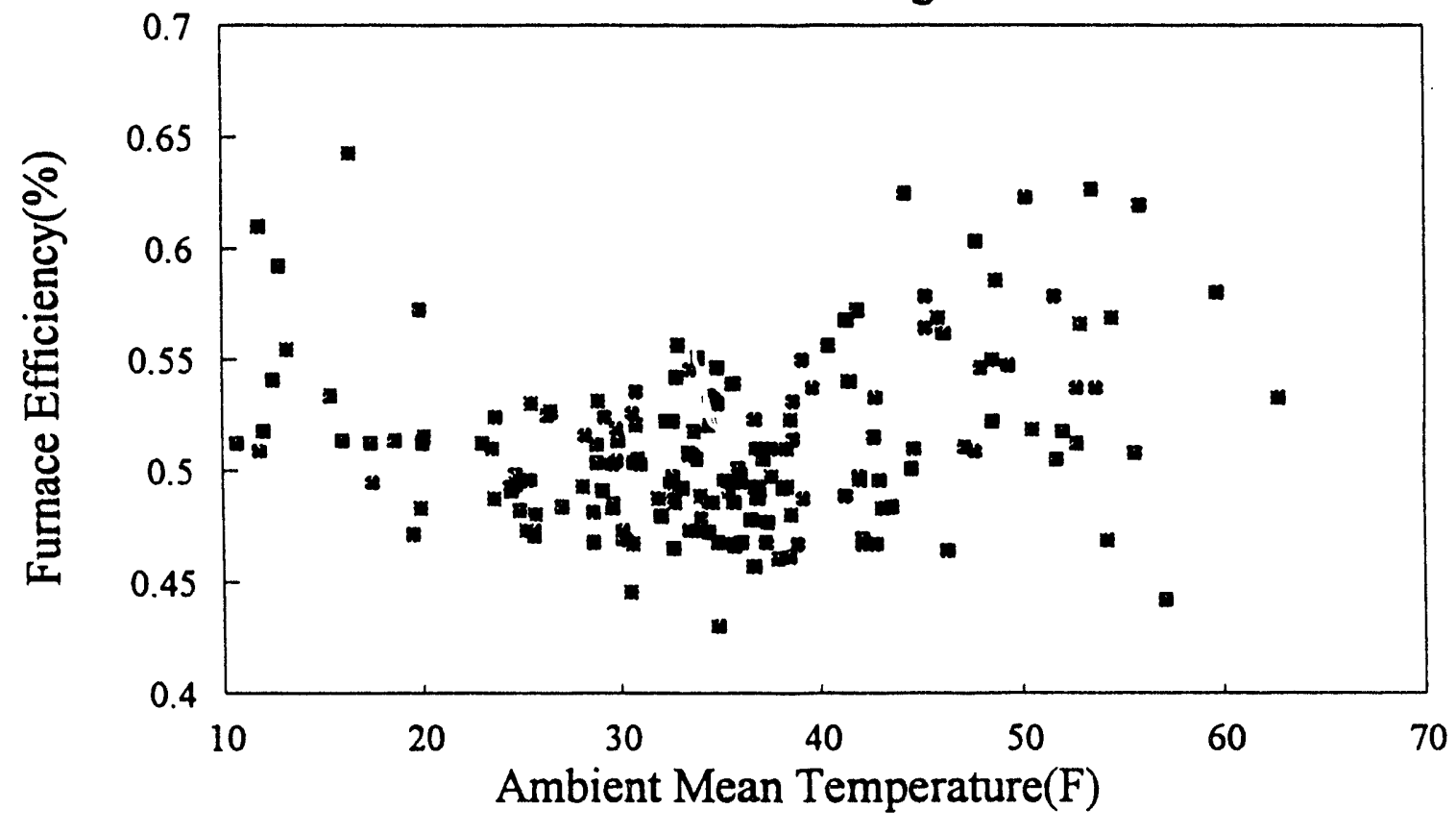

Fig. 3-4. Predicted Furnace System Efficiency Showing Little Sensitivity to Mean Ambient Temperature. 


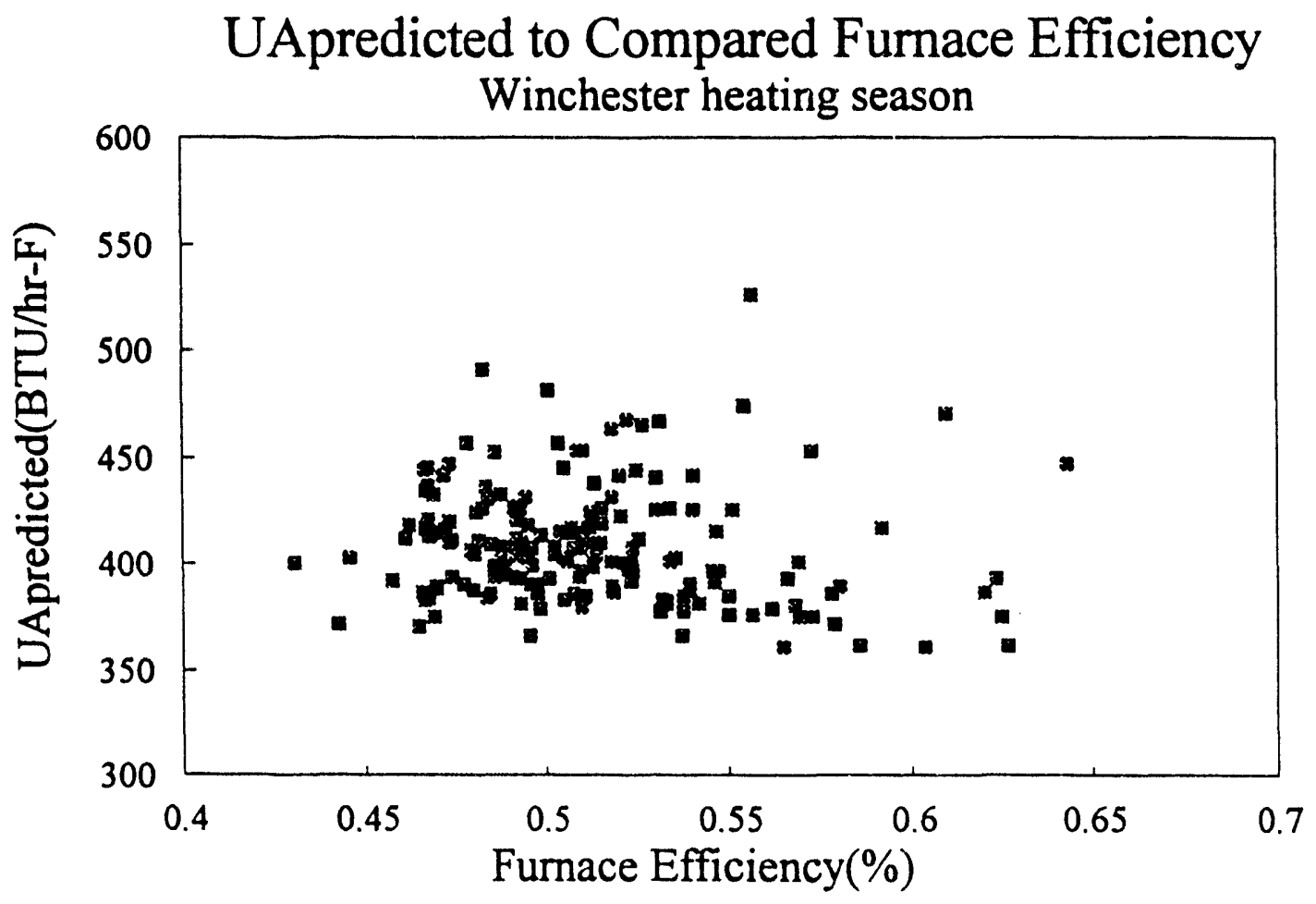

Fig. 3-5. Predicted UA Compared to Predicted Furnace Efficiency. 


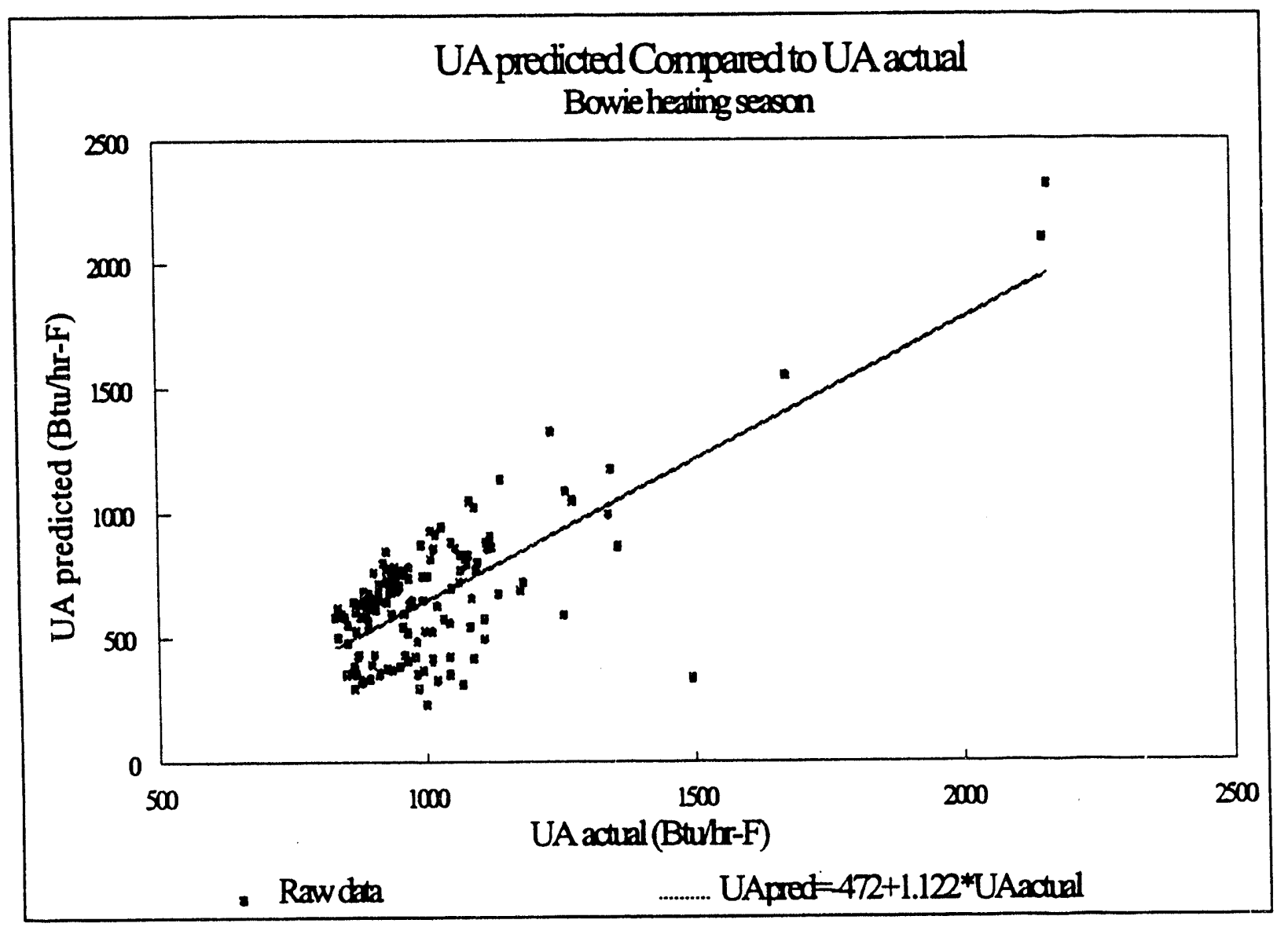

Fig. 3-6. Predicted Heat Loss coefficient Compared to the simulated Actual UA for the Bowie House during the Heating season. 


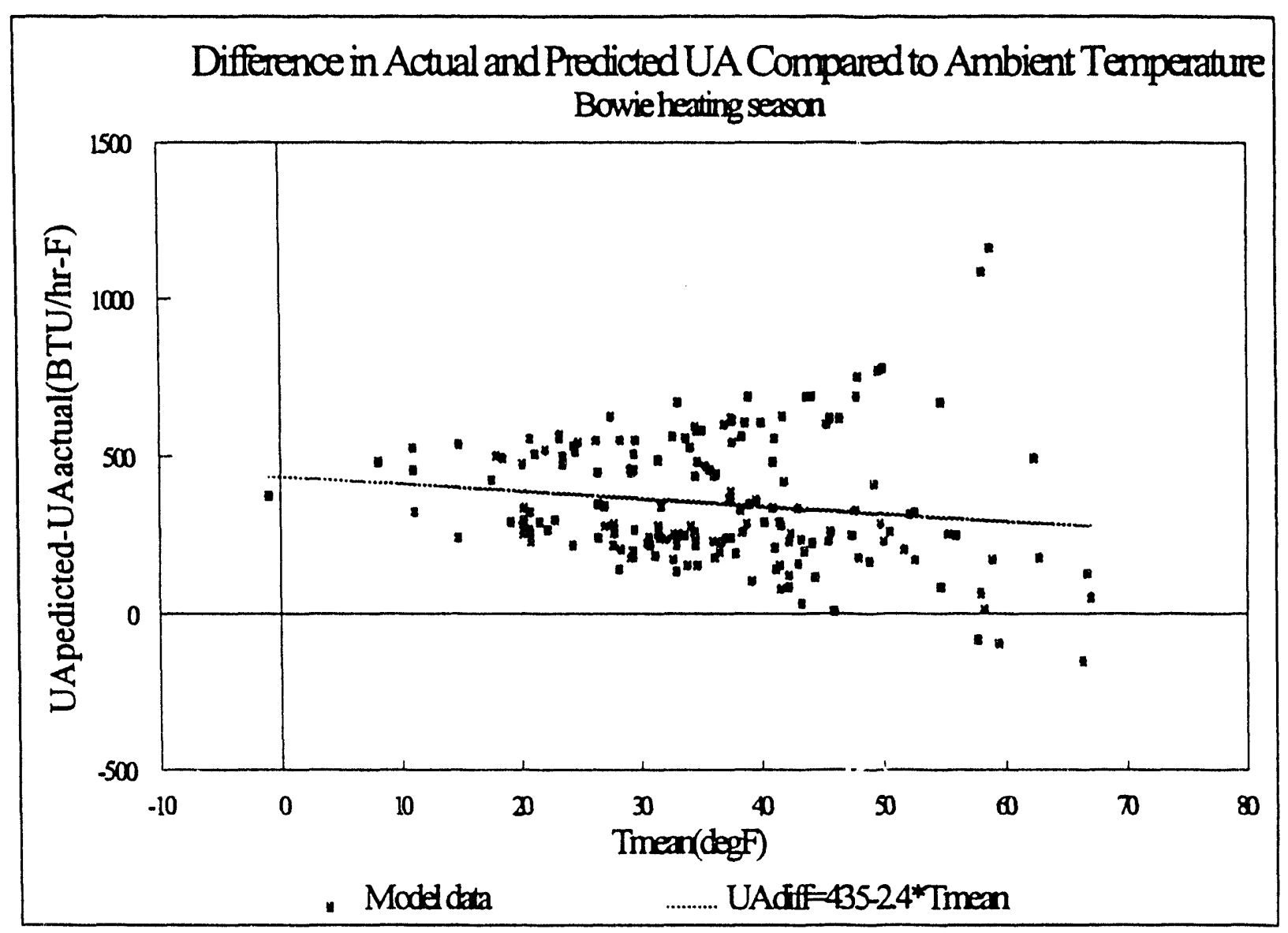

Fig. 3-7. Sensitivity of the Bowie Difference in Actual and Predicted UA to Mean Ambient Temperature during the coheat Test. 


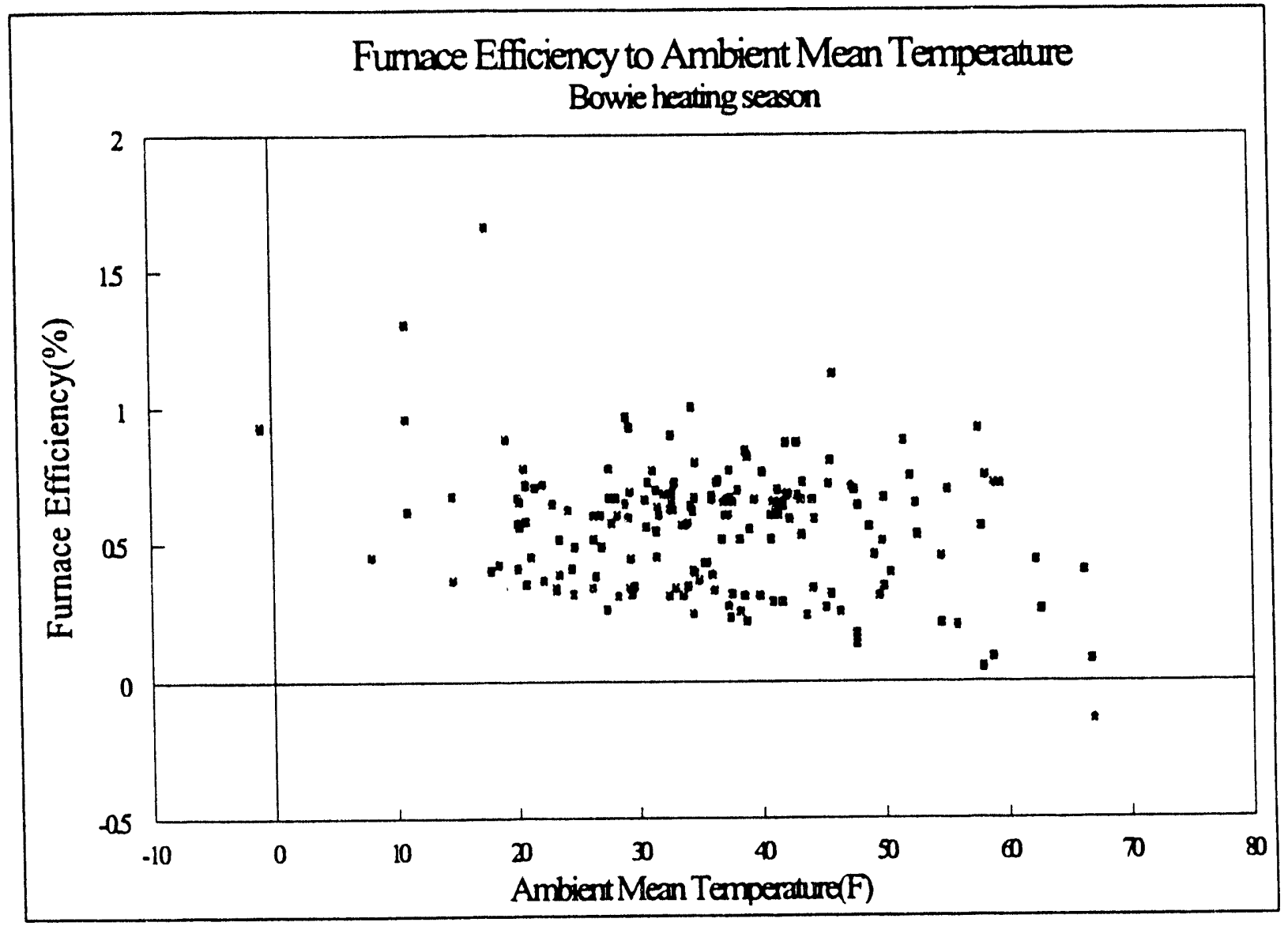

Fig. 3-8. Bowie Predicted Furnace Efficiency Related to Ambient Temperature. 


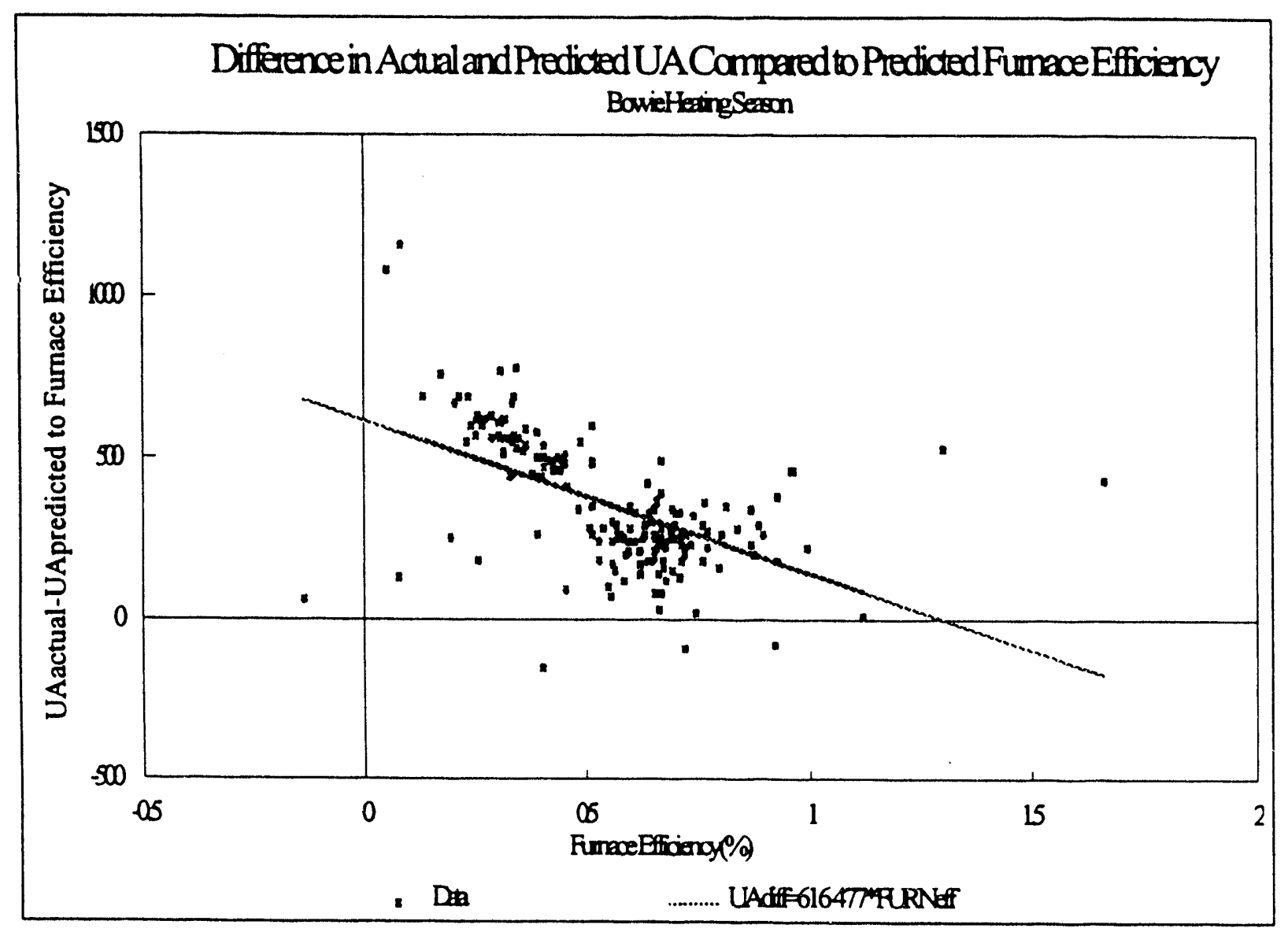

Fig. 3-9. Relationship between the Difference in Actual and Predicted UA and the Predicted Furnace Efficiency for the Bowie Heating season simulations. 


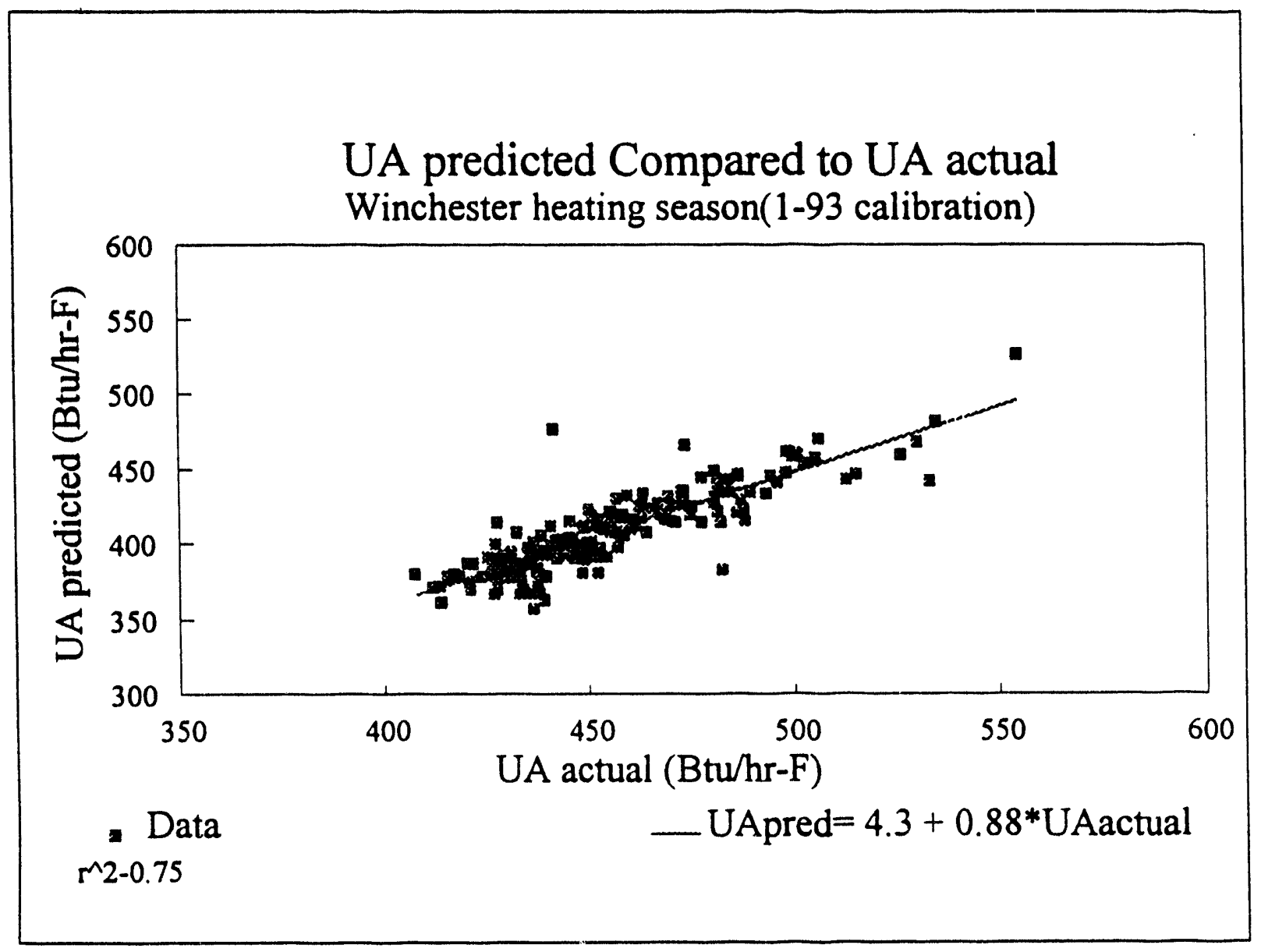

Fig. 3-10. Recalibrated Winchester Model Predicted UA Compared to Actual UA Heating season Values. 


\section{SECTION 3 REFERENCES}

Box, G.E.P., W.G. Hunter, and J.S. Hunter, 1978, 8tatistics for Experimenters, Wiley, New York.

Duffie, J.A., and W. Beckman, 1991, Bolar Energy of Thermal processes, Wiley, New York.

Hsieh, 1988, 8olar Energy Engineering, Prentice-Hall, Englewood Cliffs, NJ.

John, P.W., 1990, statistical Methods in Engineering and Quality Assurance, Wiley, New York.

NAHB Research Center, 1991, "Measured Performance Rating System," NAHB/RC, Upper Marlboro, MD.

Quinlan, F.T., 1979, "SOLMET, Vol. 2, Final Report," National Climatic Center, U.S. Dept. of Commerce, Ashville, NC. 
Section 4.0

WINCHESTER HOUSE MONITORING DATA

The second approach to uncertainty analysis is to compare MPR-based predictions of furnace fuel use to measured fuel use for the winchester house. To that end, an elapsed time meter was installed on the furnace in the winchester house in January, 1992 to capture the cumulative time of the furnace gas valve being on. Readings were taken roughly once a week of the furnace elapsed time meter, the gas meter, the electric meter, and a thermoneter in the thermostat in the house. In all readings spanned the time from January 21 to April 28, 1992, or 99 days or almost exactly half the heating season. The readings are listed in Appendix $B$. In the house, gas provided energy to the domestic hot water heater (in the basement), the dryer (in the basement), and the stove/oven (in the kitchen). Weather data from the same period was obtained from the state climatologist, R. Lautzenheiser: dry-bulb temperature in Reading, MA (approximately 3 miles north of the winchester house), wind speed and minutes of sunshine at Logan airport in Boston (approximately 10 miles south of the Winchester house).

From the weather data and the winchester house meter readings, the following estimates were made of the energy balance for the 99-day period:

(a) Total degree days: 3447 F-day, based on the thermostat average readings of $69.1 \mathrm{~F}$ and the daily average outside temperature readings.

(b) Furnace input fuel: 68.0 million Btu, based on elapsed time meter, several one-time gas flow rate measurements ( 1.158 cubic feet/minute), and a heating value of gas of 1020 Btu/cubic foot from the gas utility company invoice.

(c) Total gas use: 79.7 million Btu based on the utility meter readings and the heating value of gas given in (b). 
(c) Total electricity used: 8.2 million Btu, based on the utility meter readings.

(d) Internal gains estimate: 9.39 million Btu, based on the sum of the electricity use (virtually all of electrical use is in the house and eventually turns to heat) and of ten percent of the gas not used in the furnace (studies indicate that roughly this percentage becomes internal gains from domestic hot water use and from dryer use--SERI, 1984)

(e) Solar input: 3.4 million Btu, based on minutes of sunshine and model given in Lunde (1980), transmittance of 0.7 , and 54 square feet of almost-south-facing glass.

The infiltration component of heat loss is estimated to be that of the heating season average predicted value from the MPR tests and the analysis described in our report of June 1991 to NAHB/RC.

It is assumed that $\mathrm{NAHB} / \mathrm{RC}$ will do the actual comparison of predicted and measured furnace energy use so as to minimize any possibility of predicting the "right answer." We have not made any prediction ourselves as to the furnace use.

SECTION 4 REFERENCES

Lunde, P., 1980, Solar Thermal Engineering, Wiley, New York, p. 507 .

SERI, 1984, "Passive Solar Performance, Summary of Class B Results," Solar Energy Research Institute report SERI/SP-271-2362, Golden, CO. 
Section 5

SUMMARY AND OBSERVATIONS

The following strategy was used in estimating the sensitivity and uncertainty in the MPR method: model two houses with widely-varying thermal characteristics; perform simulated MPR tests with an entire heating season of representative weather data, keeping the thermal characteristics constant; and perform simulated MPR tests with varying thermal and test parameters, keeping the weather data constant. In the last set of simulations, experimental design methods were utilized to minimize the number of simulation runs but not to eliminate possible interactions of parameters.

The results for the winchester house were in general consistent with the repeated actual MPR test results (as reported in NAHB/RC, 1991). A 10\% bias (underprediction) and a $3 \%$ random uncertainty were obtained in UA estimates over one heating season with TMY data. For weatherization "before and after" tests, a change in $7 \%$ in UA should be detectable at a $95 \%$ confidence level. For furnace efficiency estimates, a bias (overprediction) and a random uncertainty of both roughly $10 \%$ were the outcomes.

The results for the Bowie house were in general unrealistically widely varying. Although it appears that there are certainly some real sensitivities that were brought to light in the analysis of the Bowie tests, such as furnace ducts in the slab and large amounts of thermal mass, much of the scatter and bias may be more due to the model than to the MPR method. The models 
are highly dependent on calibration data. The Bowie calibration data was taken in the process of simply performing multiple MPR tests and not with the intention of collecting data for calibrating a model. More complete measured data under a set of varying experimental conditions is needed to choose the proper level of complexity of the model and to estimate the parameters. The system identification method developed by Bourque and Duffy (1992) was useful in this context for the winchester house and would be very useful for the Bowie house.

One aspect of the MPR method that should be examined as a result of the Bowie simulations is the wisdom of using a single UA to represent the thermal efficiency of a building with a slab on grade. There should be two heat loss coefficients examined separately: the UA from the heated zone to the ambient air and the UA from the heated zone to the earth. In the Bowie simulations, we included the equivalent UA of the slab to earth in the overall "actual" UA by taking the UA of the slab to earth and multiplying it by the ratio of the temperature difference of the average inside, and the earth to the temperature difference of the average interior and the ambient. Sometimes this ratio was huge when the difference in interior and ambient temperatures became very small. In a couple of cases during the heating season simulations, the equivalent UA of slab to earth was over $1000 \mathrm{Btu} / \mathrm{h}-\mathrm{F}$. In the majority of cases during the simulated heating season, the slab UA components comprised over one half the total "actual" UA. The apparent difference between the MPR predicted and the "actual" UA was in great part due to high slab loss. The MPR method should be extended to predict both the UA to air and the UA to earth, separately. A somewhat simplified version of the analytic approach of Bourque and Duffy (1992) with a series of coheat, furnace, and cooldown tests would probably be adequate to predict both UA components. Of course, more study would be needed here. 
Based on the results of developing a complex model and of performing many simulations of the MPR tests on the model of the winchester house, the following observations can be made: It appears that the MPR method is reasonably precise for large field applications of the method (for which it was originally intended). In weatherization applications, UA changes as low as $7 \%$ should be detectable with two carefully administered "before and after" tests. The bias in UA predictions can in part be reduced by applying the regression models developed here for sensitivity to ambient temperatures and infiltration levels. Additional bias correction equations could be developed with additional simulations and more complete experimental designs focused on the most sensitive parameters. Initial conditions at the time of the start of the tests must be monitored and accounted for in the analysis. Further development of non-steady-state models and "ramp-up" type corrections need to be undertaken. Differences between the indoor and outdoor temperatures of at least $20 \mathrm{~F}$ should be maintained by raising the indoor thermostat set points if necessary.

At this time, the overall best method of uncertainty prediction in the field still appears to be the system identification method (Bourque and Duffy, 1992) of estimating the model equation error in real time given the measurement error estimates (which are relatively more easily predicted).

One other result of this analysis is worth noting: the MPR predictions of furnace efficiencies for the winchester house model overestimated the actual by $10 \%$ on the average. Many of the MPR predictions of furnace efficiencies obtained in the field were low, with some even in the range of $30 \%$ (NAHB/RC, 1991). If in fact the actual heating system efficiencies were even lower, the implications for the need of weatherization and upgrades of heating systems and for the need of a method such as the MPR to test entire heating systems is clear. 


\section{SECTION 5 REFERENCES}

Bourque, K., and J.J. Duffy, 1992, "Building Thermal Parameter Estimation using system Identification and Short-Term Tests," Proceedings of the 1992 National Solar Energy Conference, American Solar Energy Society, Boulder, co.

NAHB Research Center, 1991, "Measured Performance Rating System," NAHB/RC, Upper Marlboro, MD. 
APPENDIX A

BOWIE AND WINCHESTER CALIBRATION PLOTS 


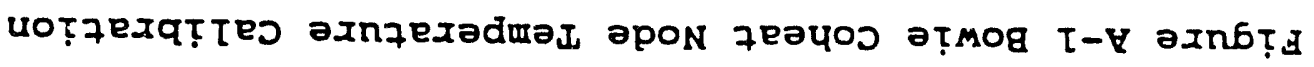

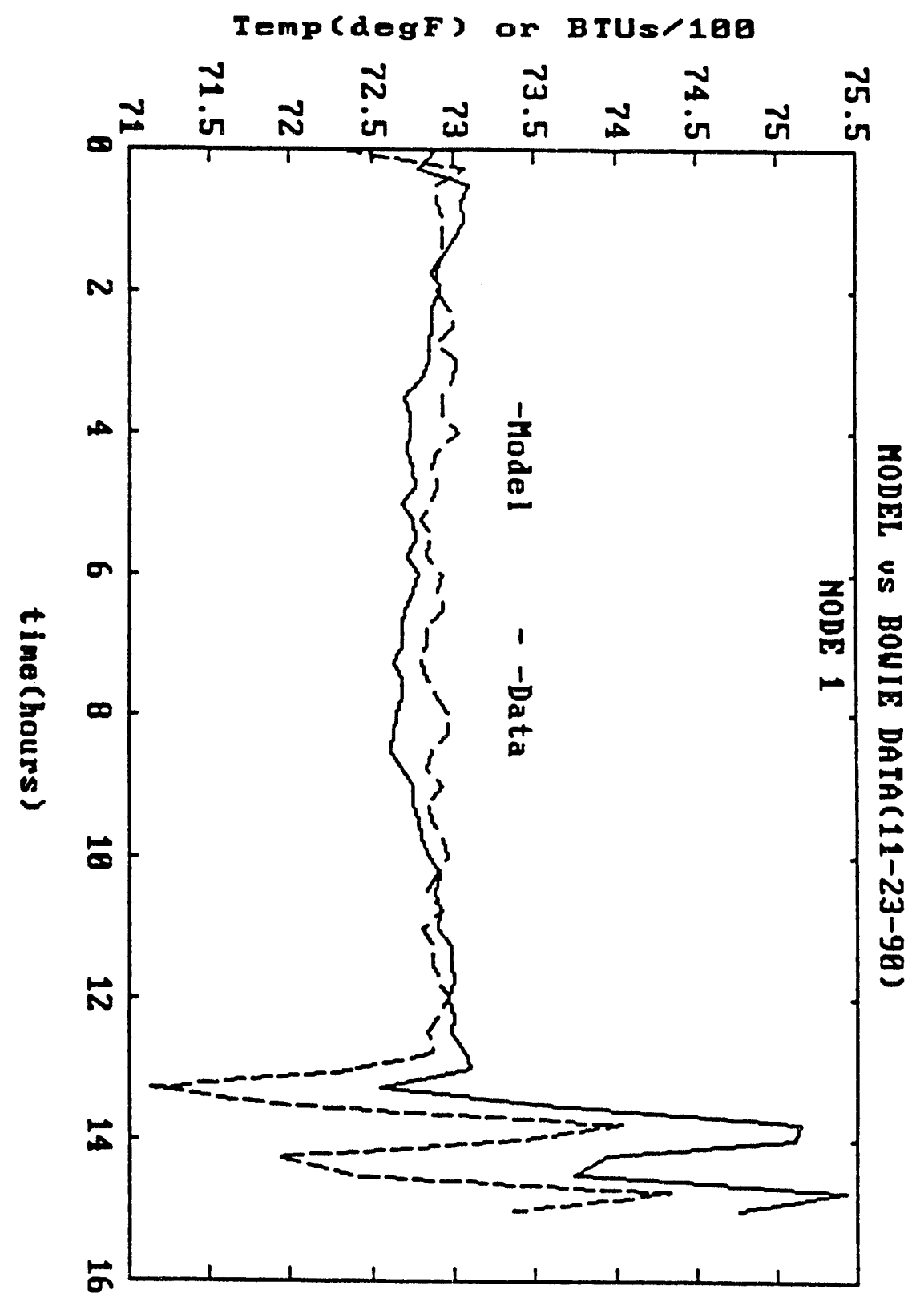


BOWIE COHEAT NODE TEMPERATURE CALIBRATION

D-75 


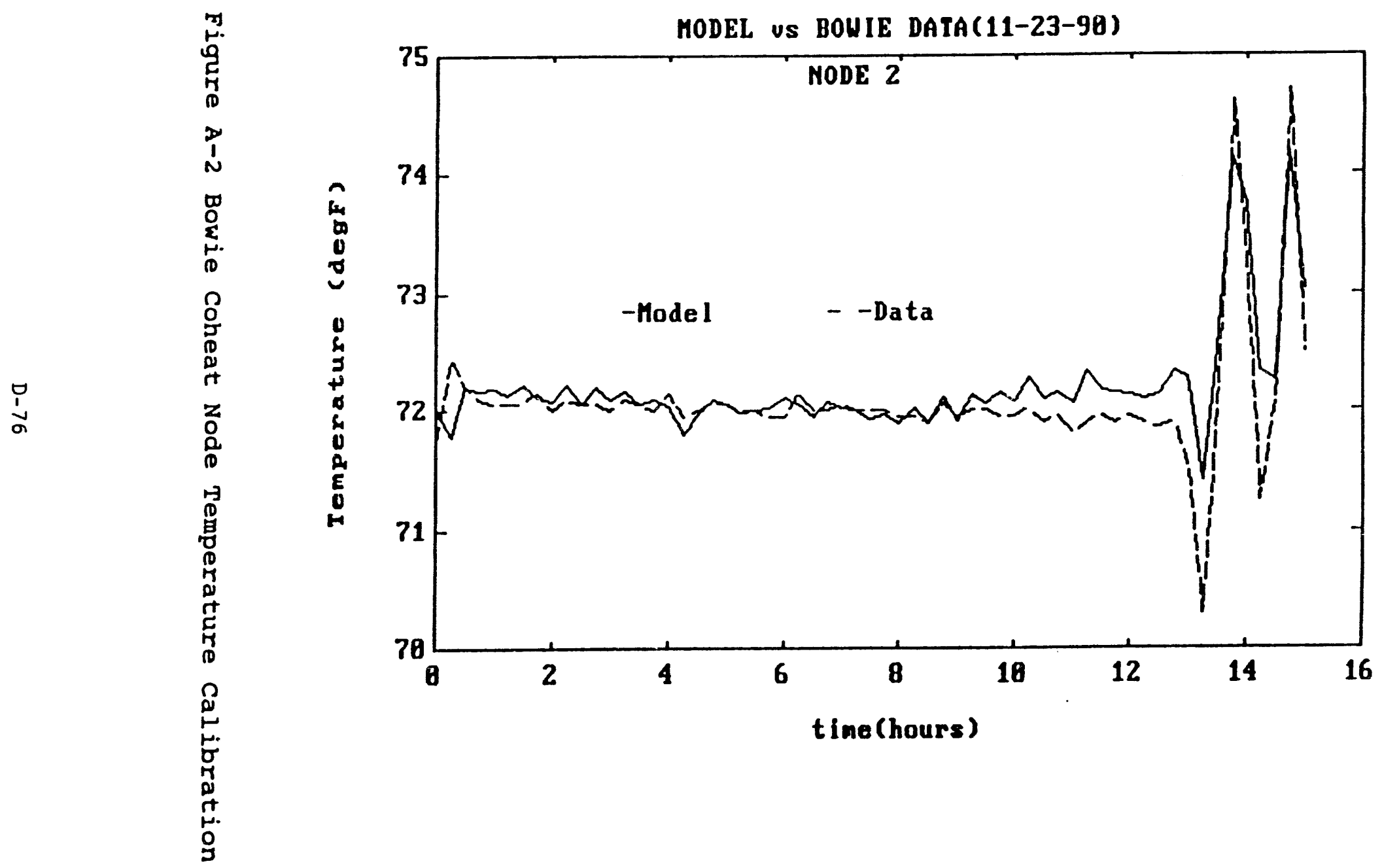




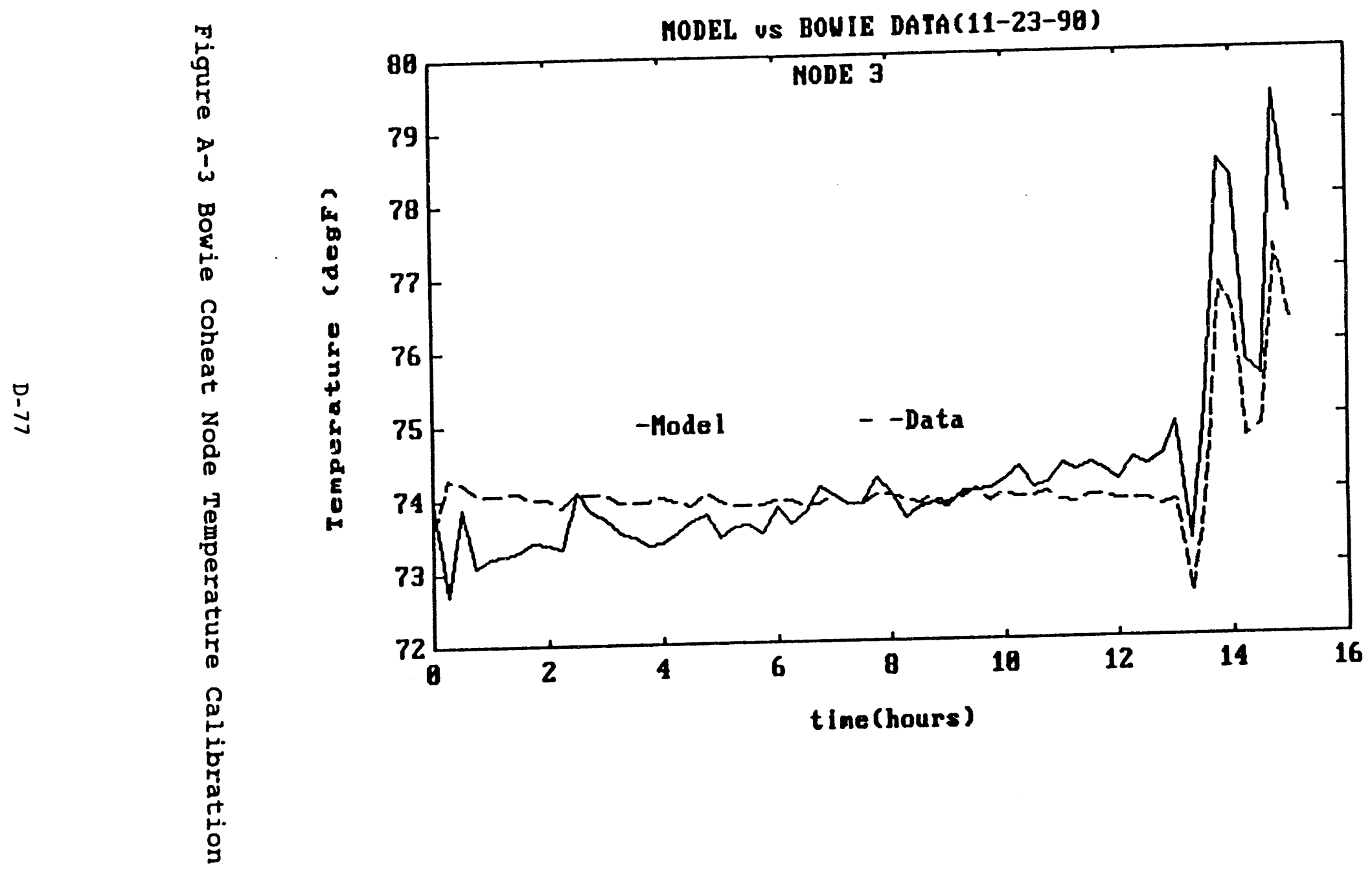




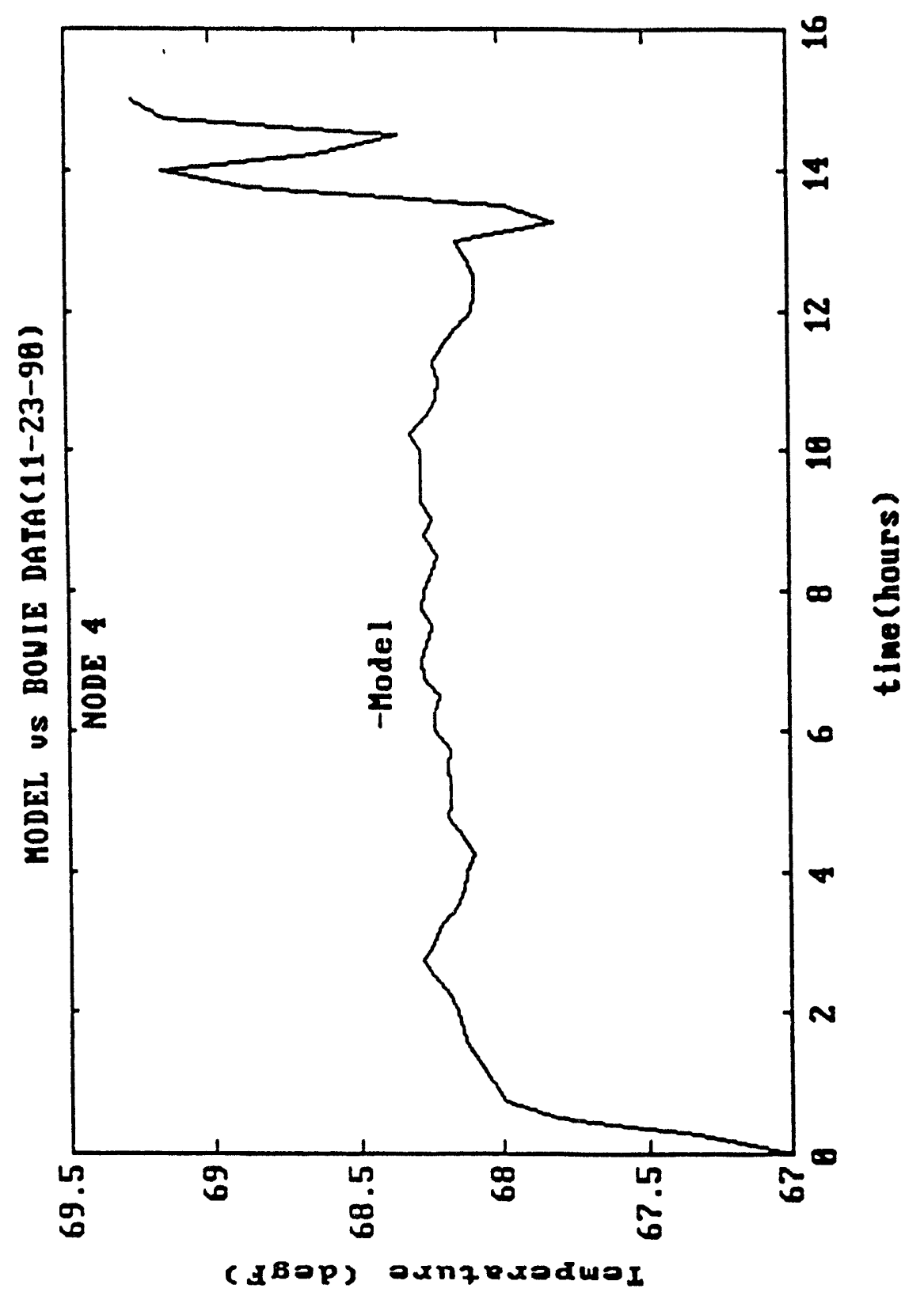

Figure A-4 Bowie Coneat Node Temperature Calibration 


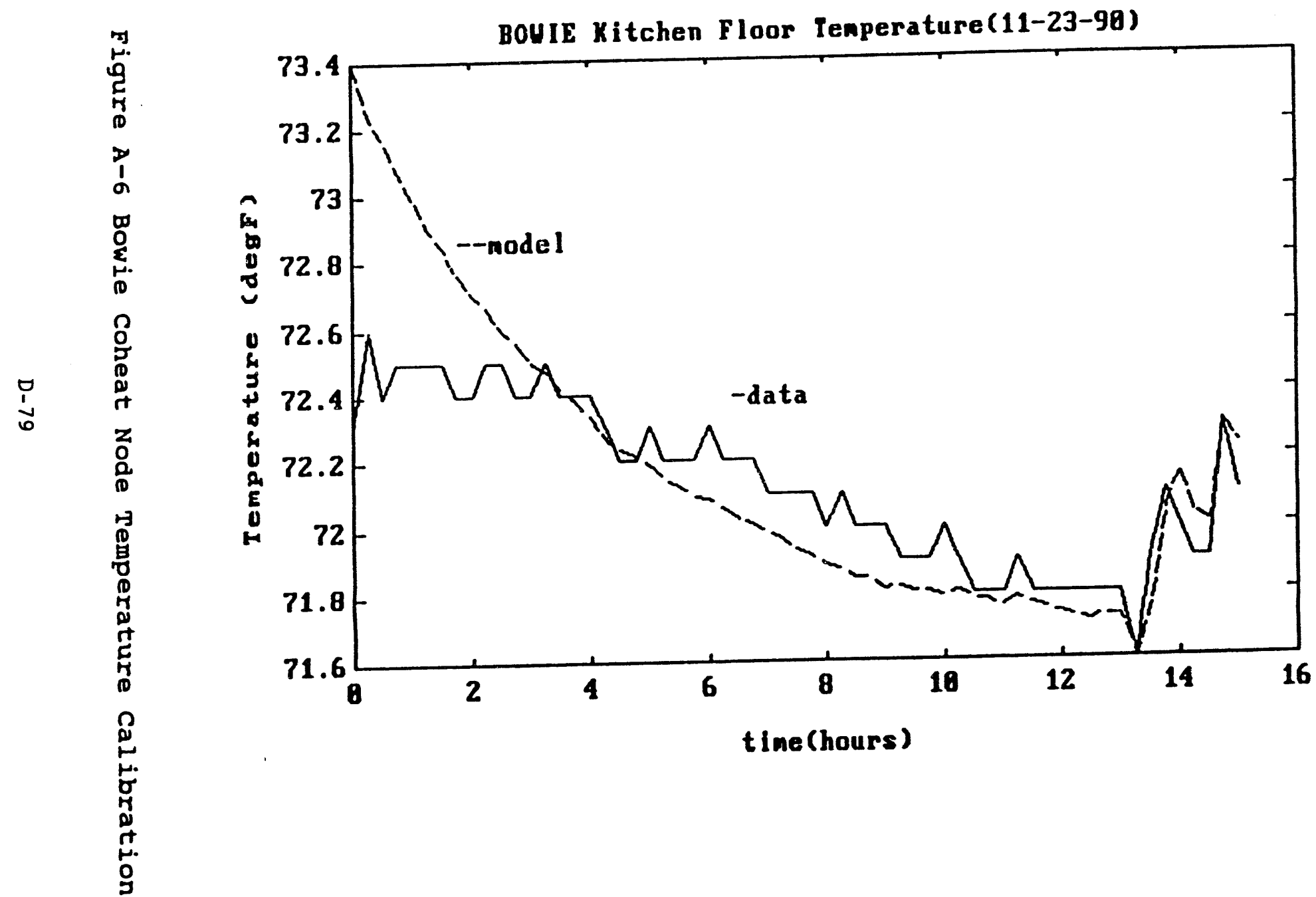




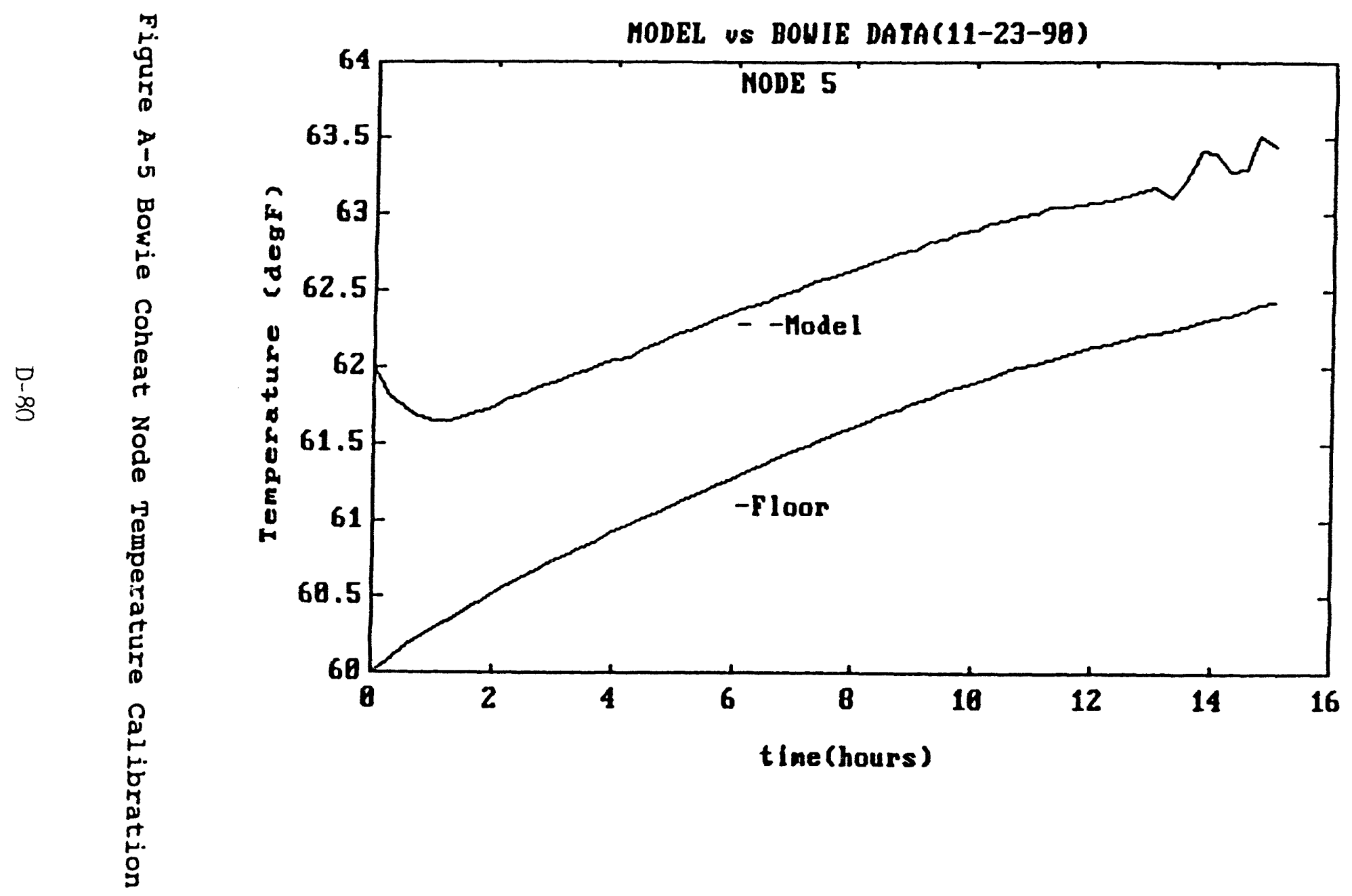


BOWIE COHEAT ENERGY BALANCE CALIBRATION

D-81 


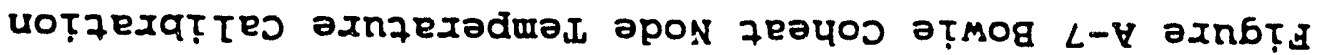

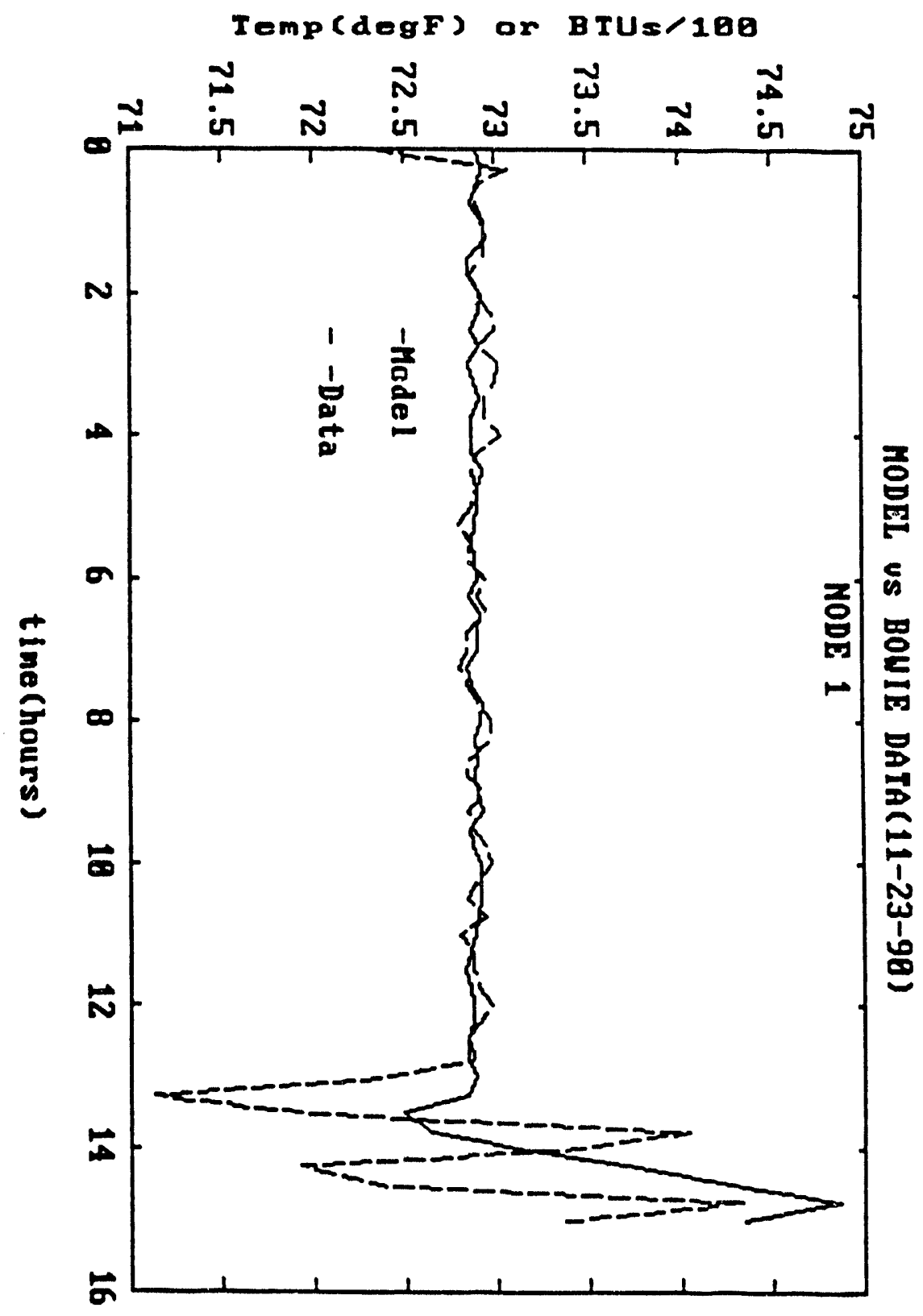




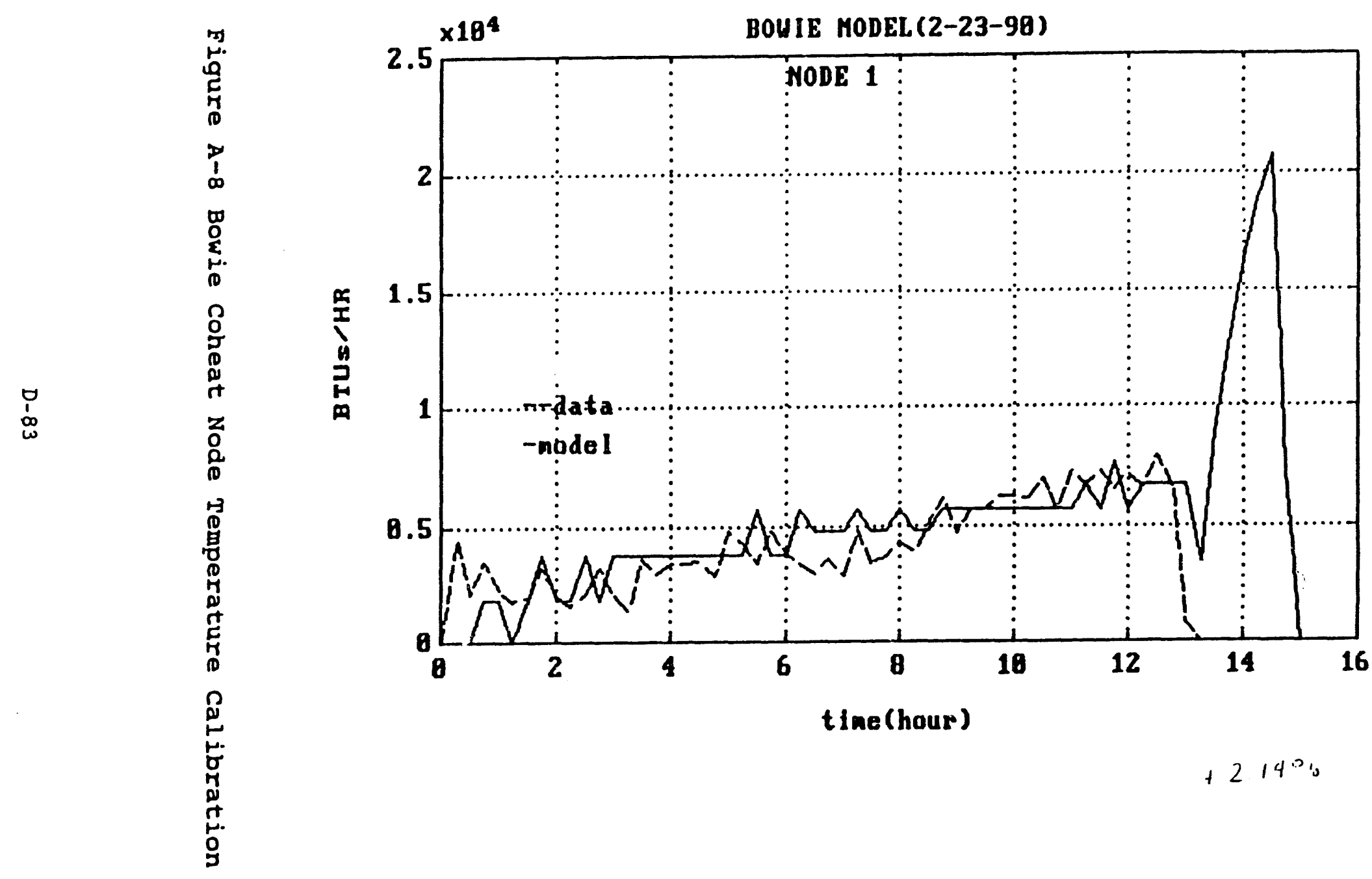




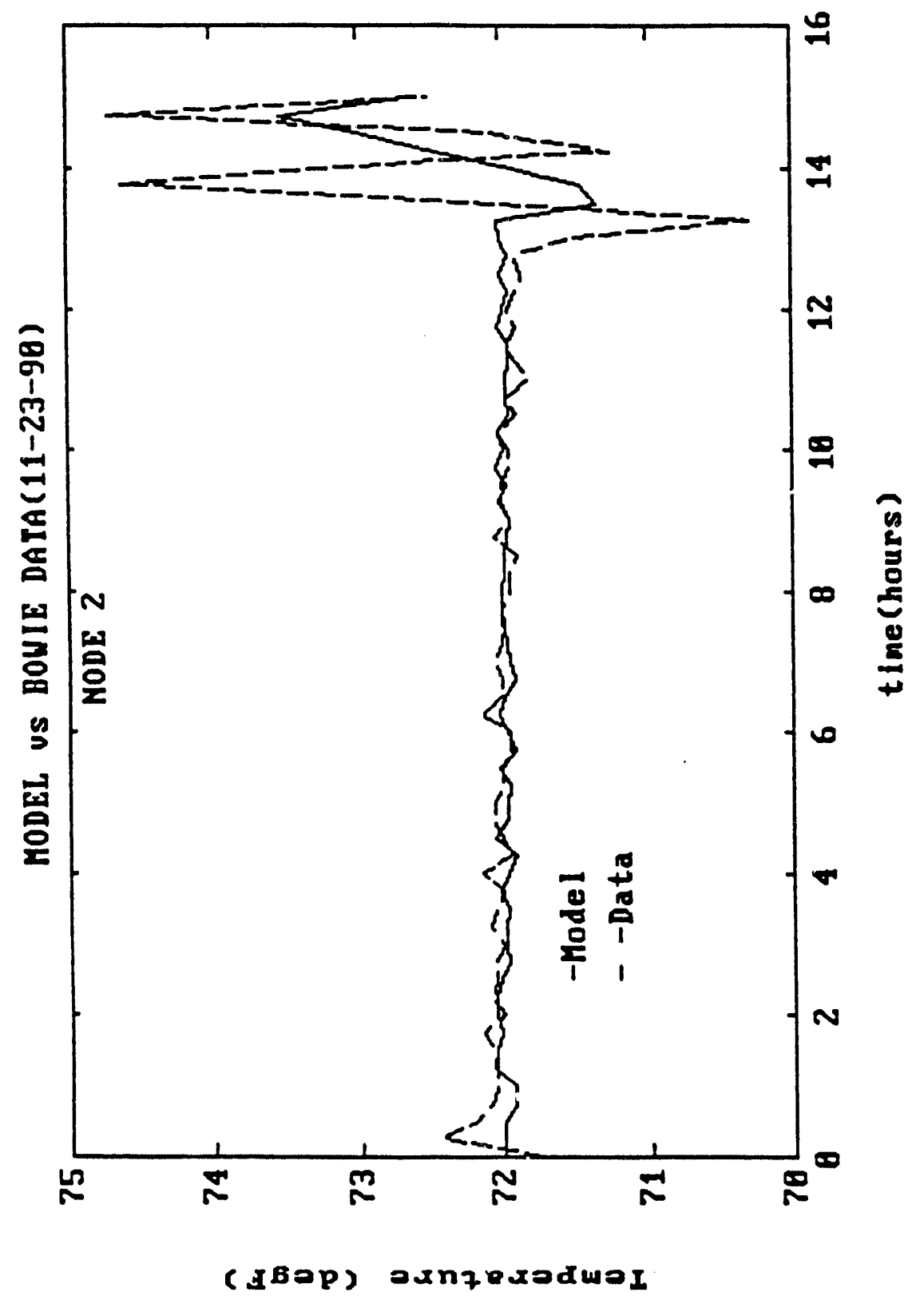

Figure A-9 Bowie Coheat Node Temperature Calibration 


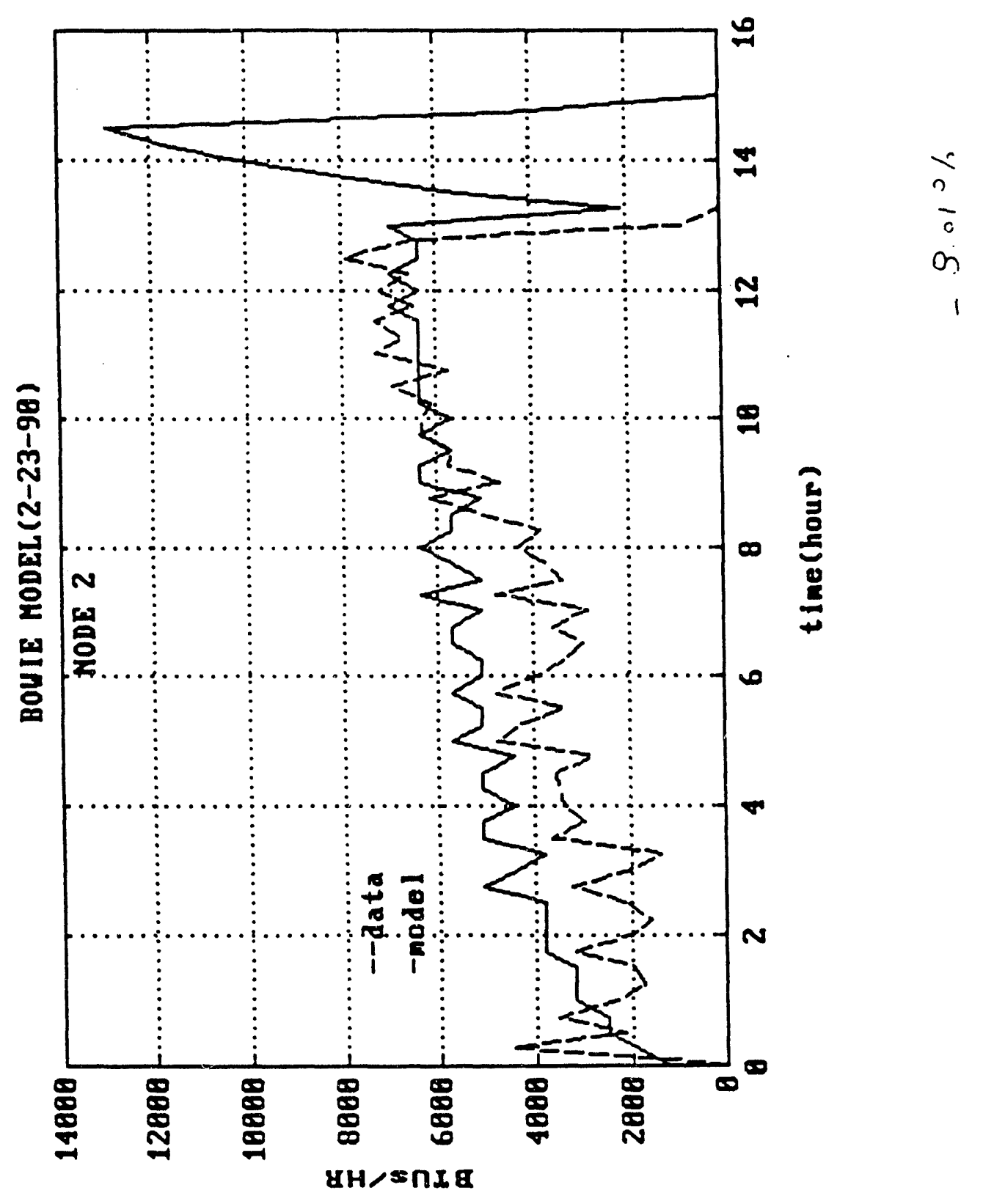

Figure A-10 Bowie Coheat Node Temperature Calibration 


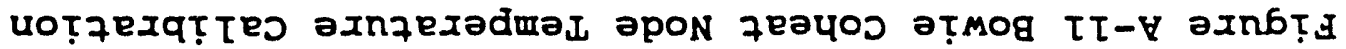

Temperature (degF)

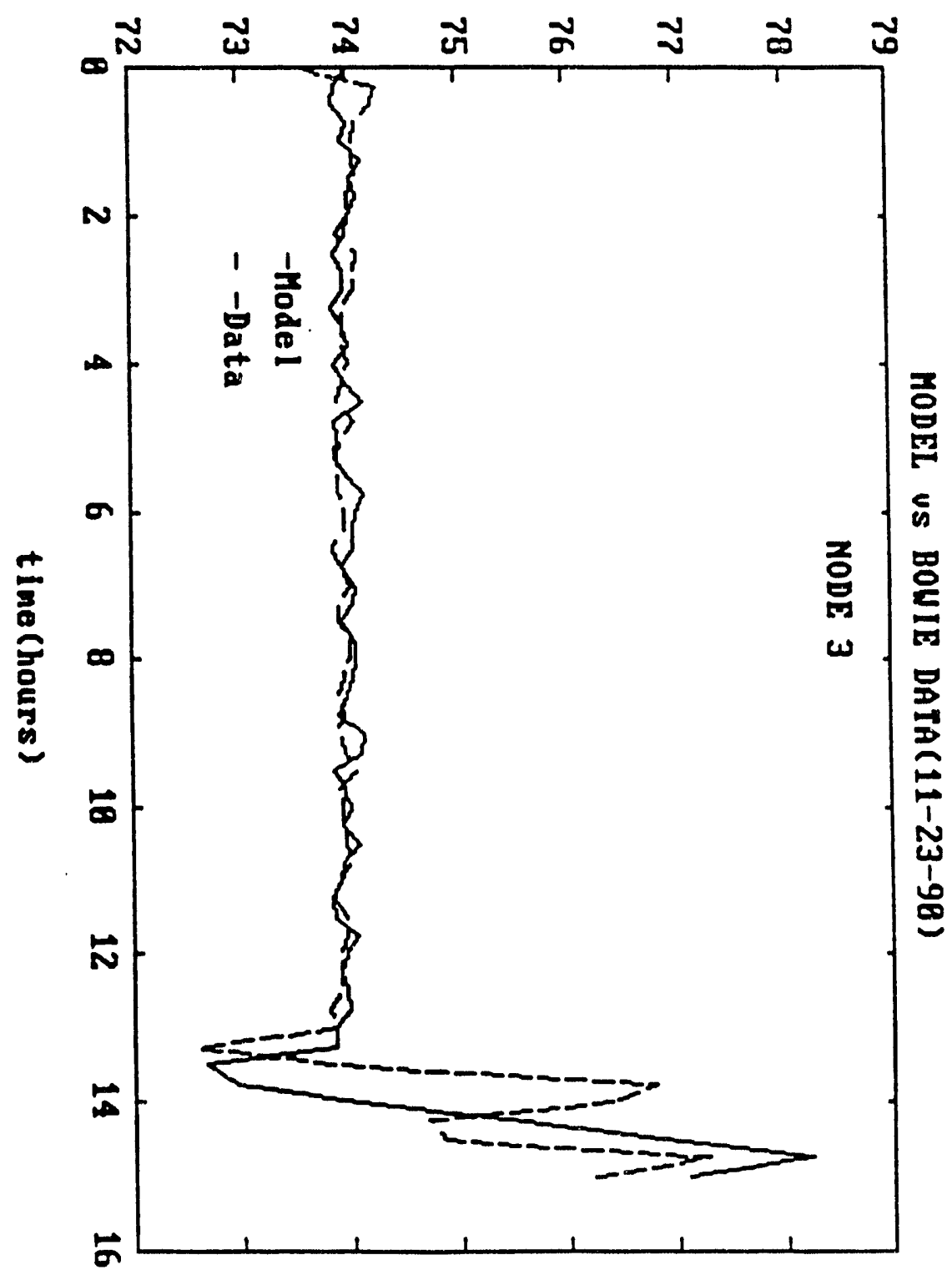




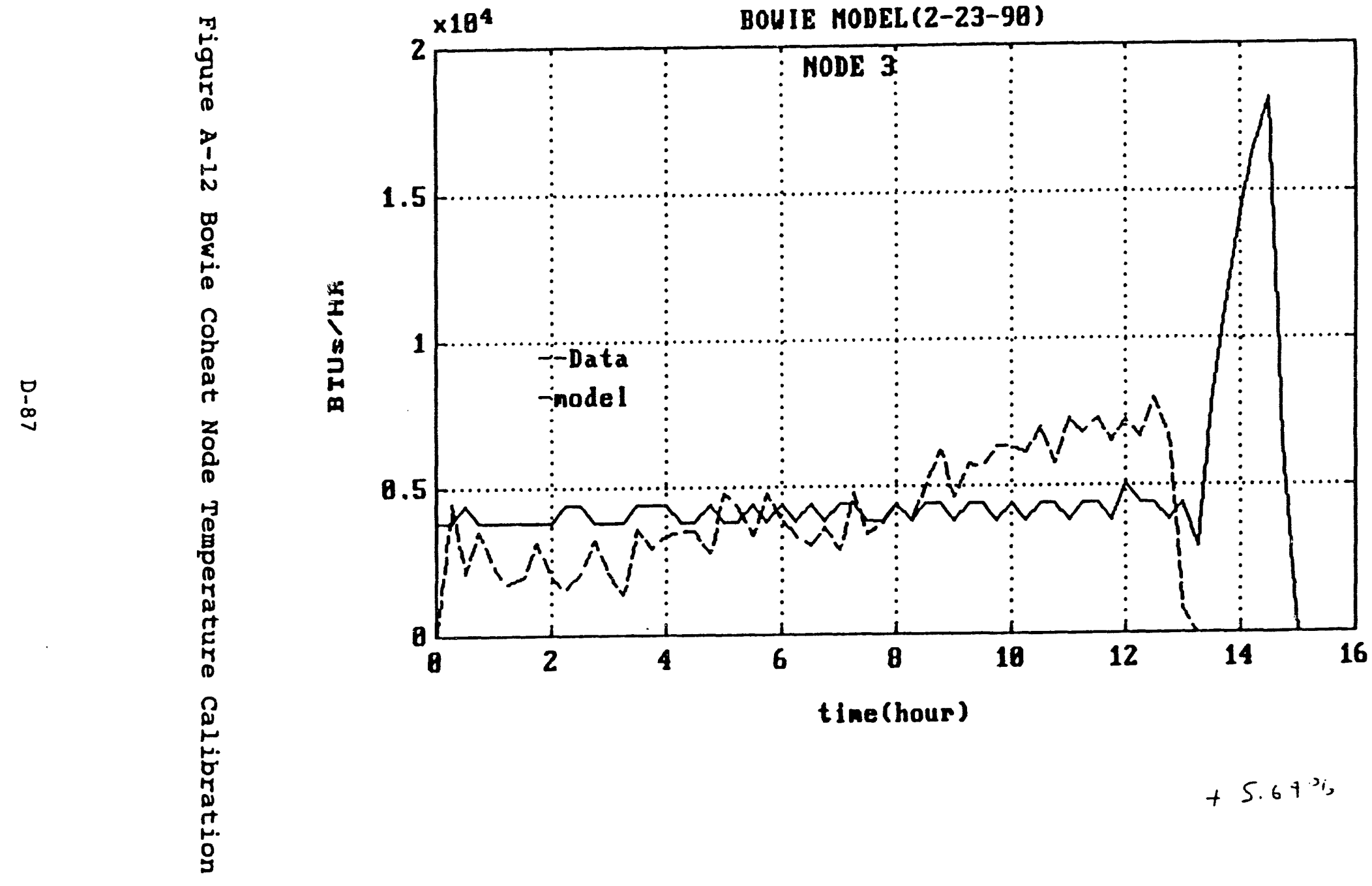




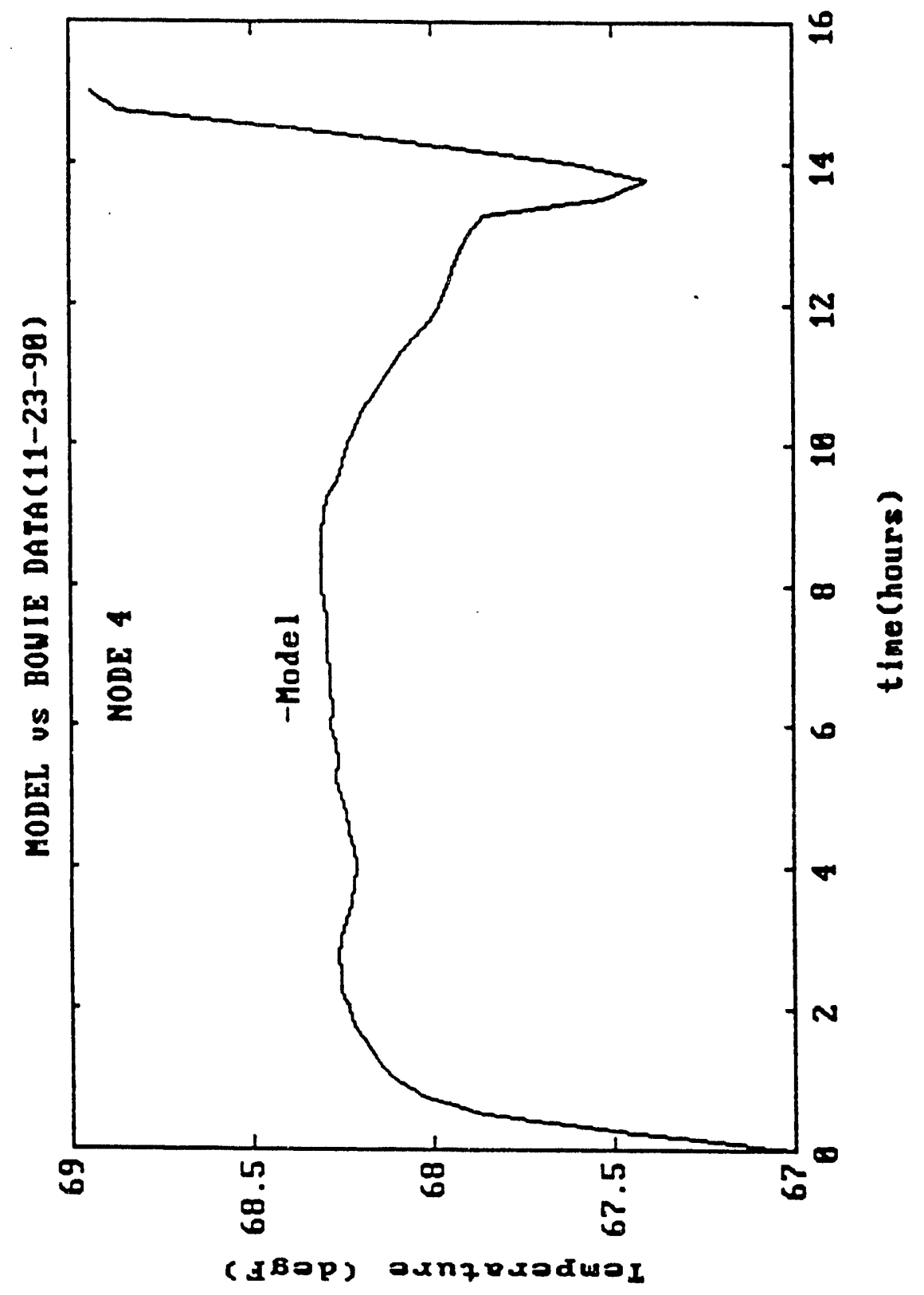

Figure A-13 Bowie Coheat Node Temperature Calibration 


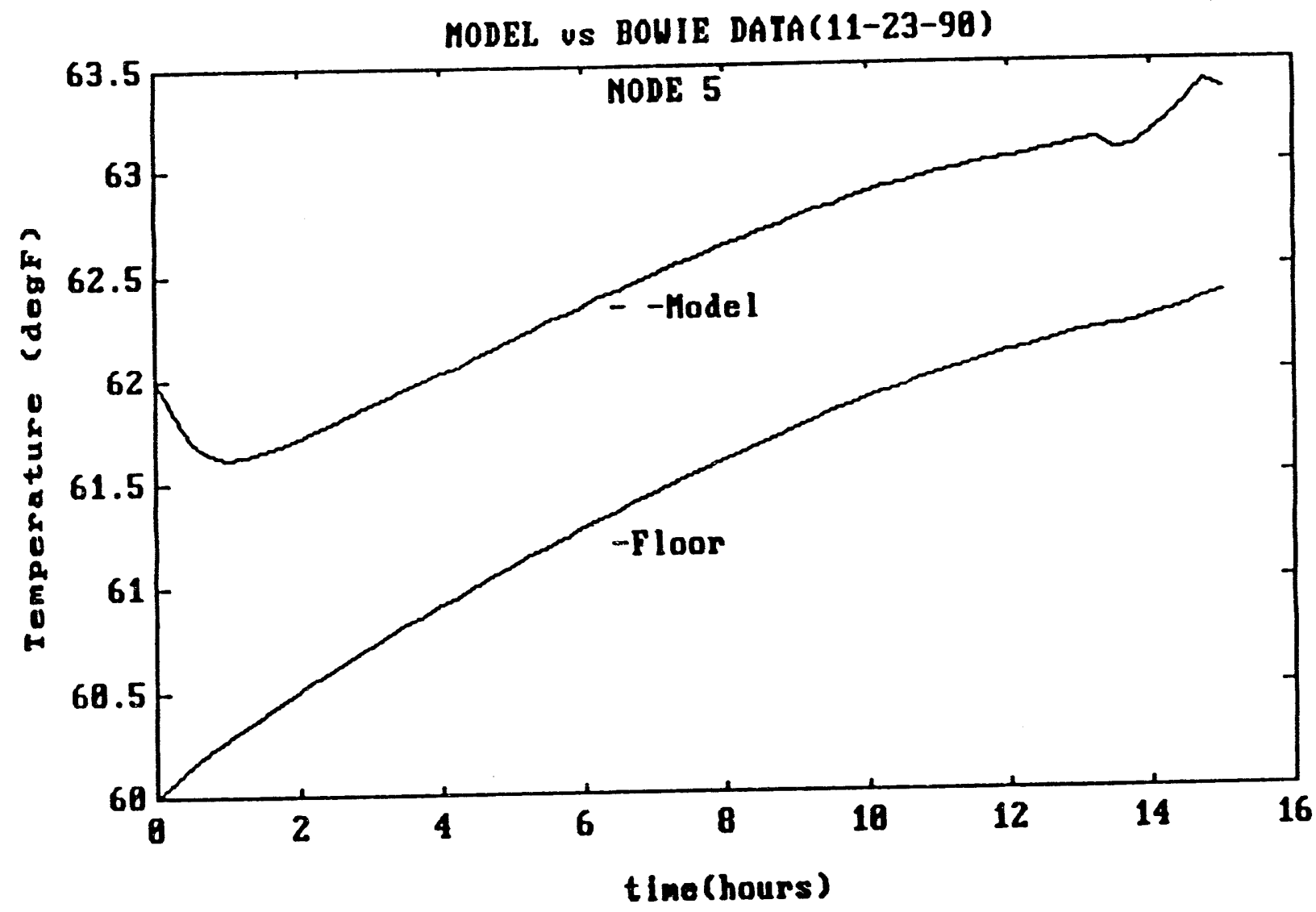




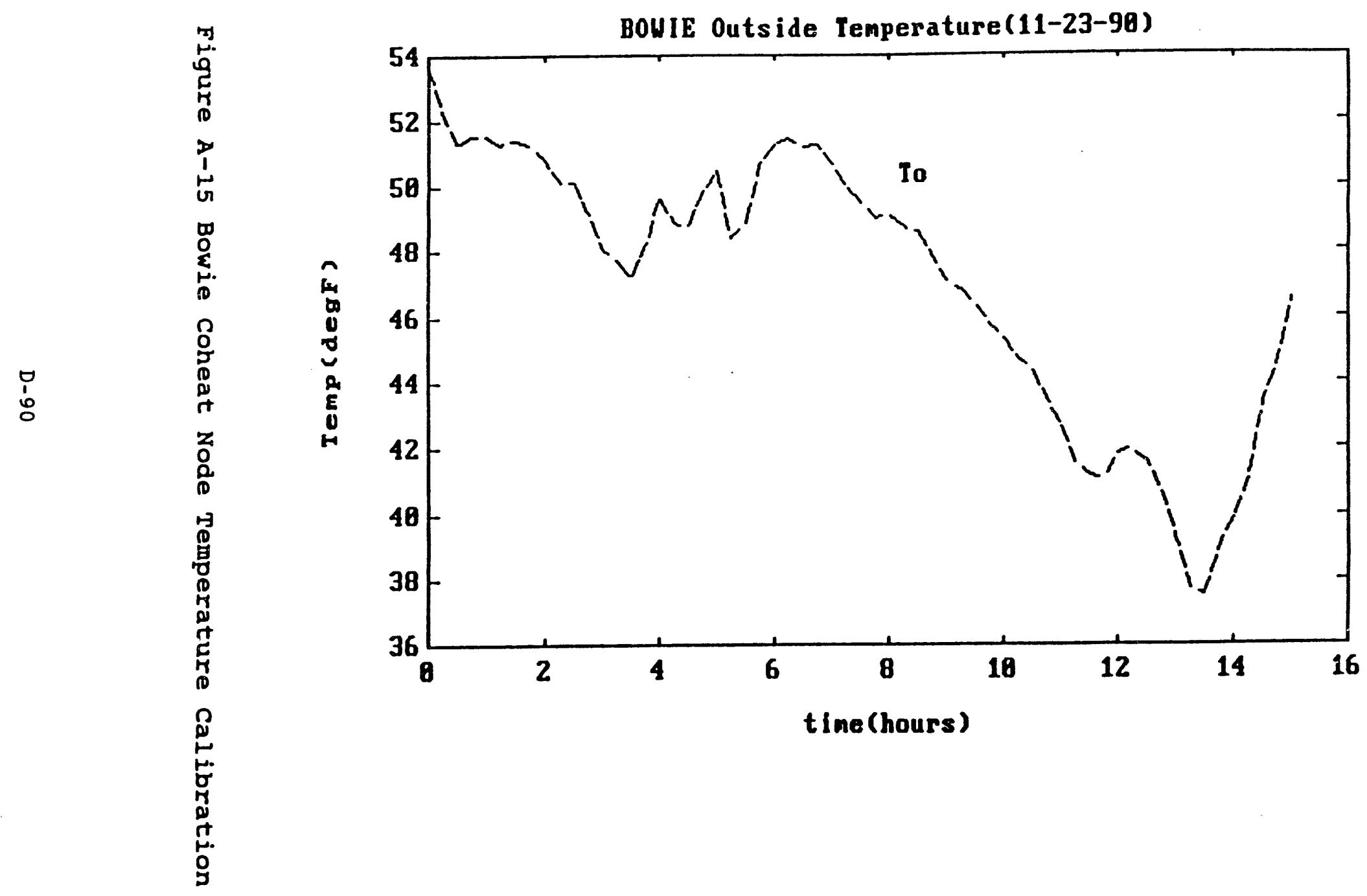




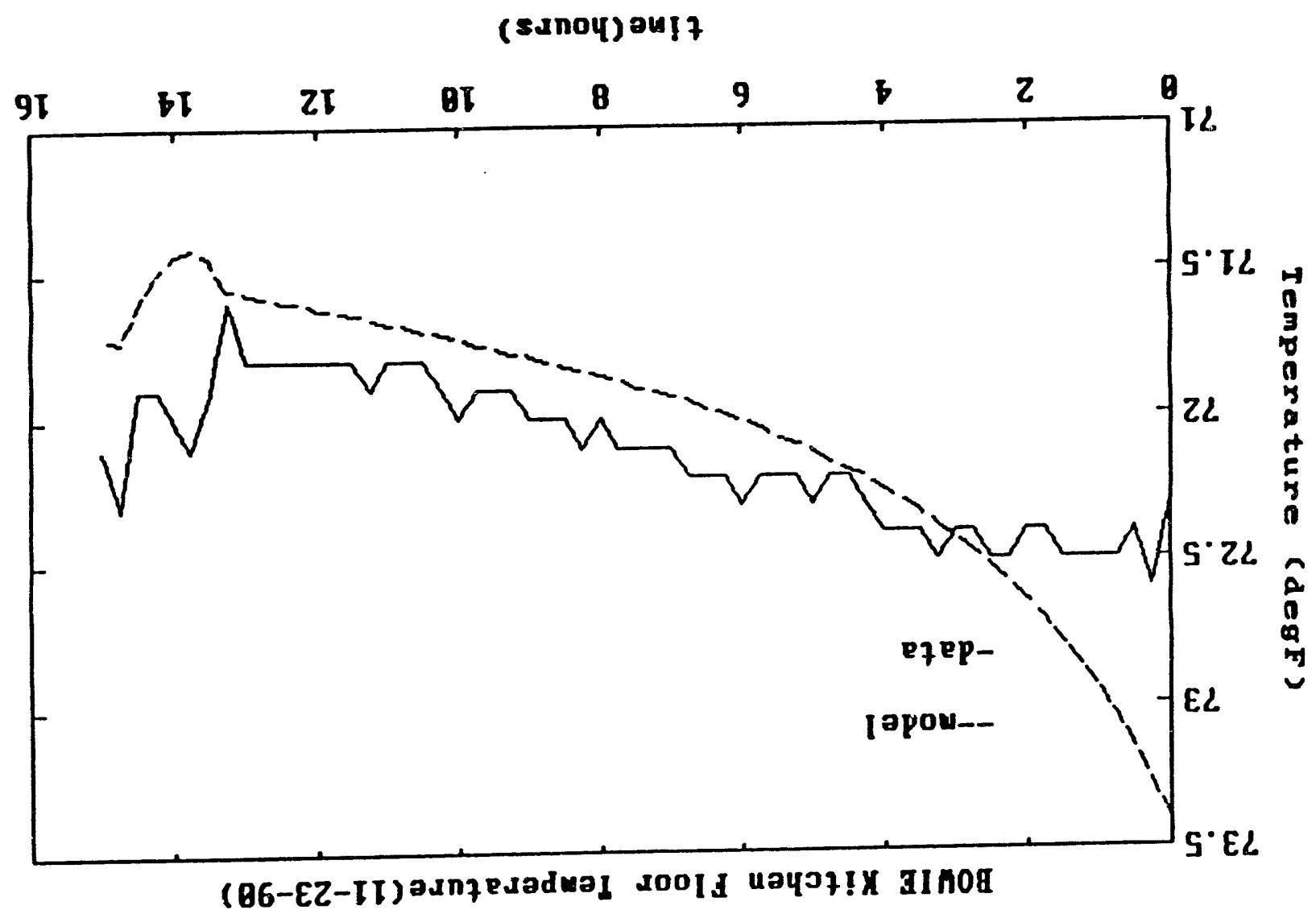

ó 
BOWIE FURNACE DISTRIBUTION

AND

EFFICIENCY CALIBRATION

D-92 


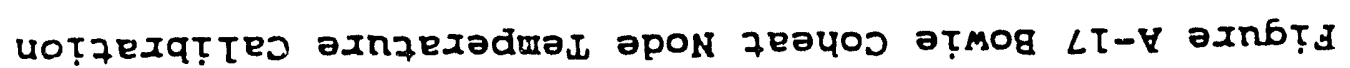

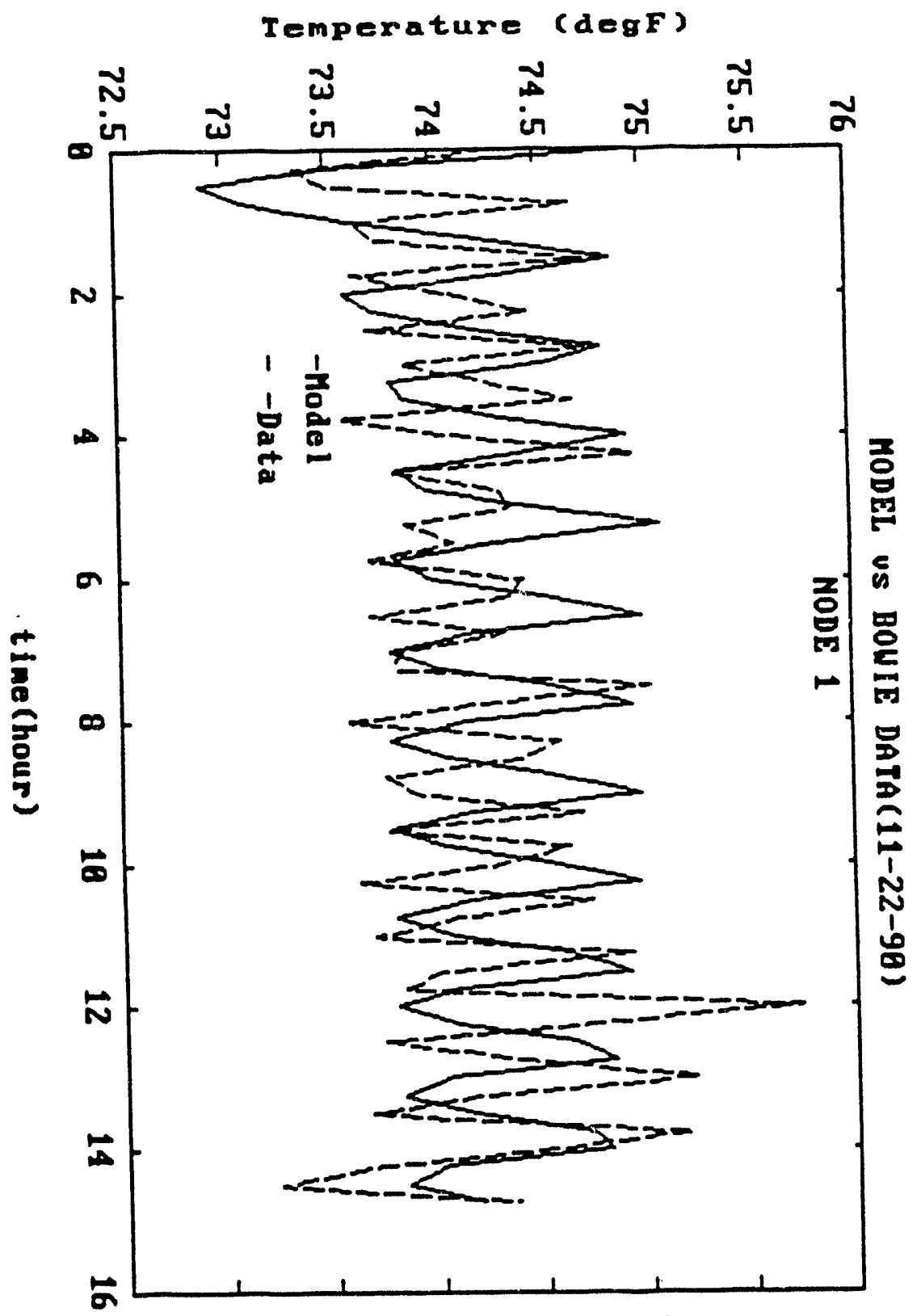




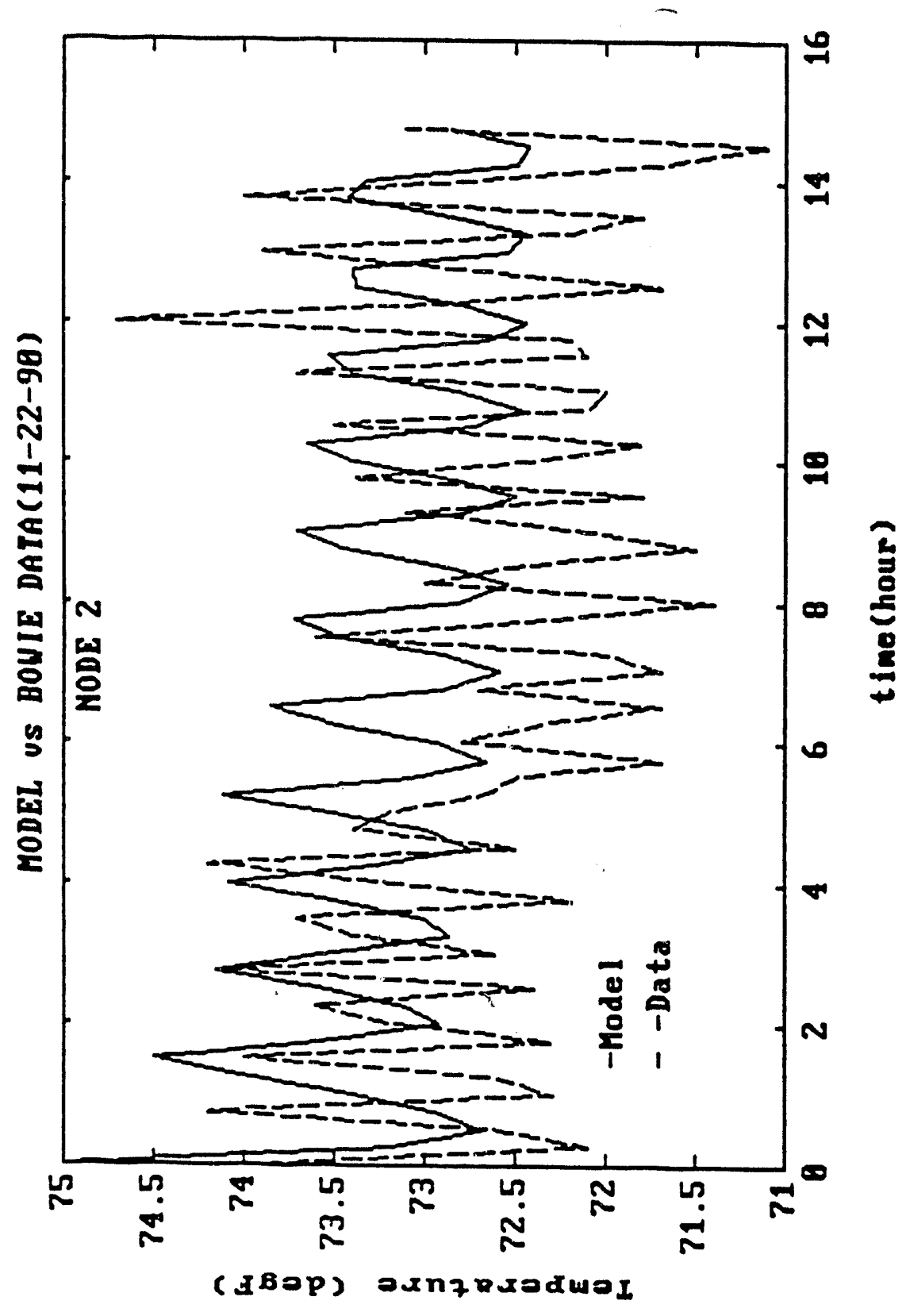

Figure A-18 Bowie Coheat Node Temperature Calibration 


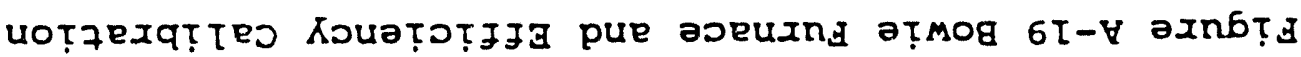

Temperature (degF)

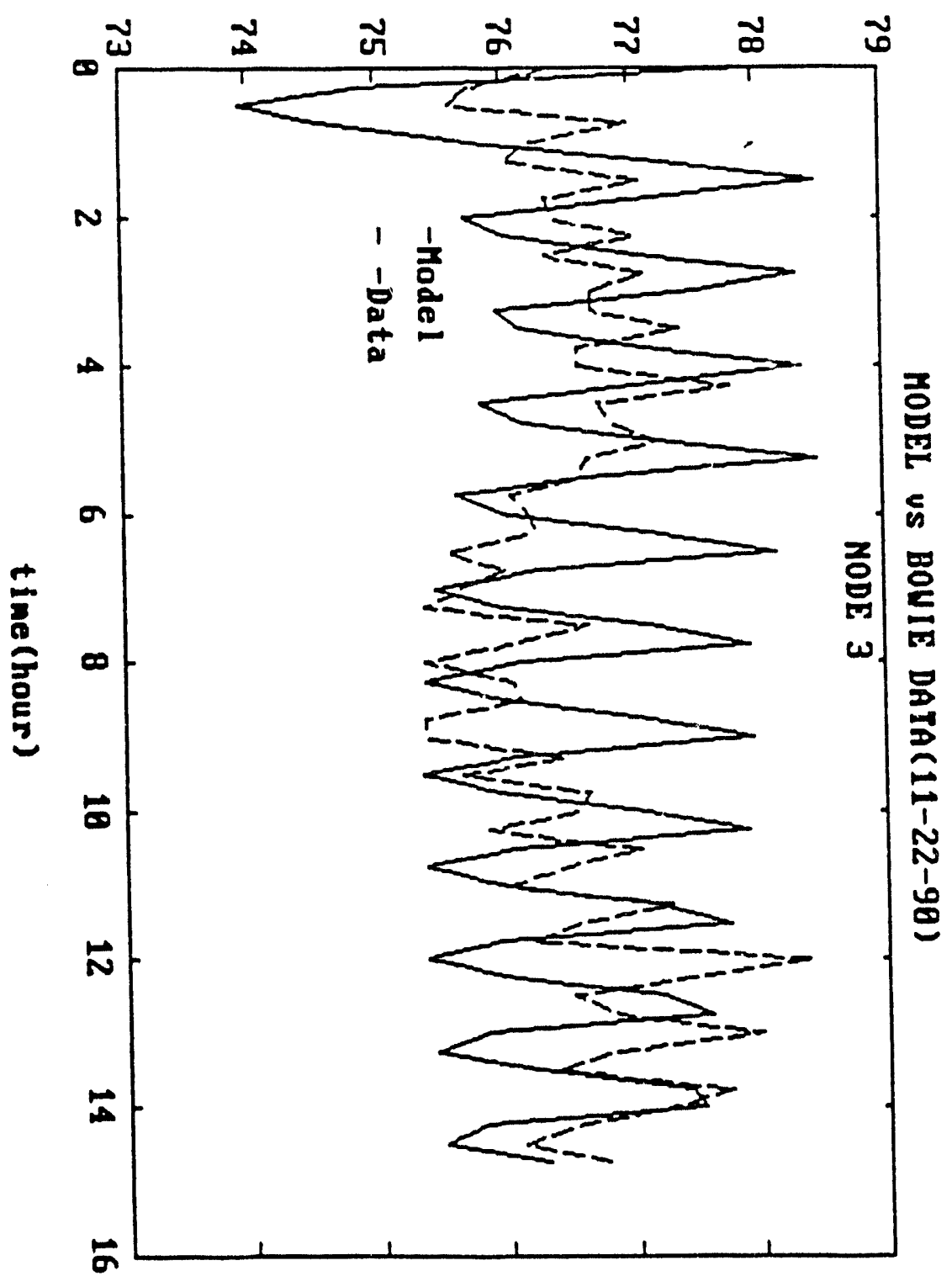


WINCHESTER CALIBRATION PLOTS

D-96 
WINCHESTER NODE TEMPERATURE CALIBRATICN

$$
\text { D-97 }
$$




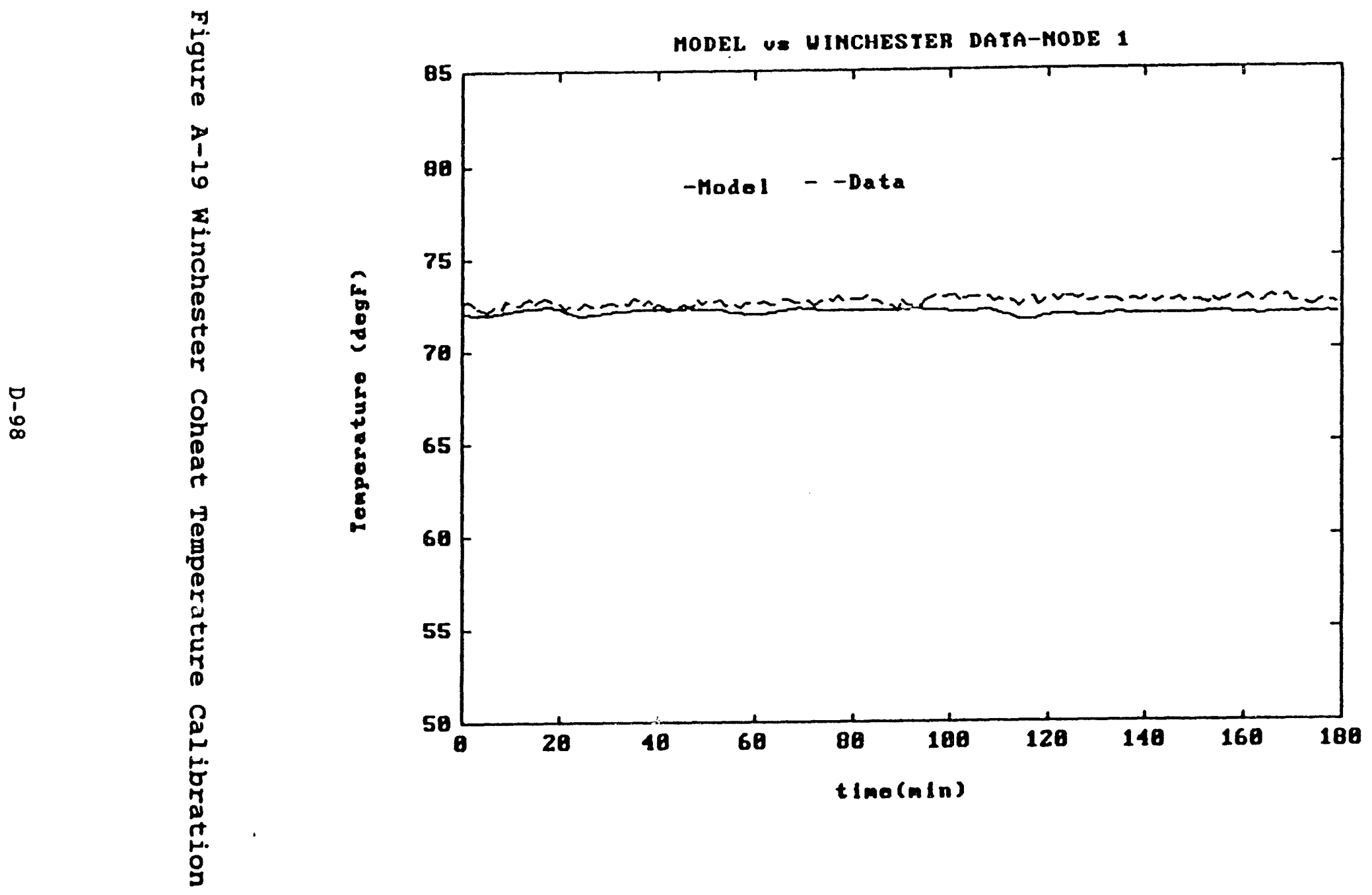




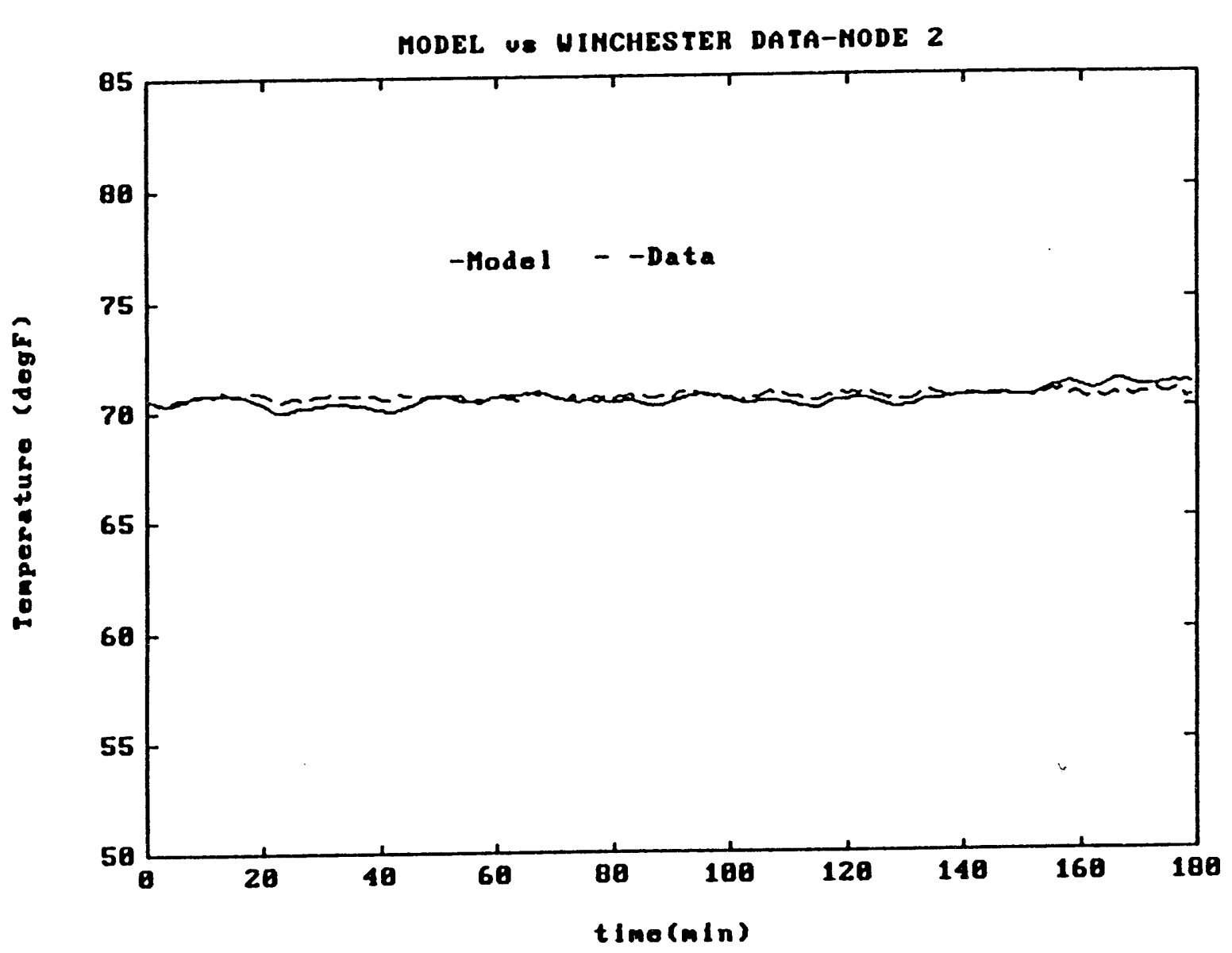




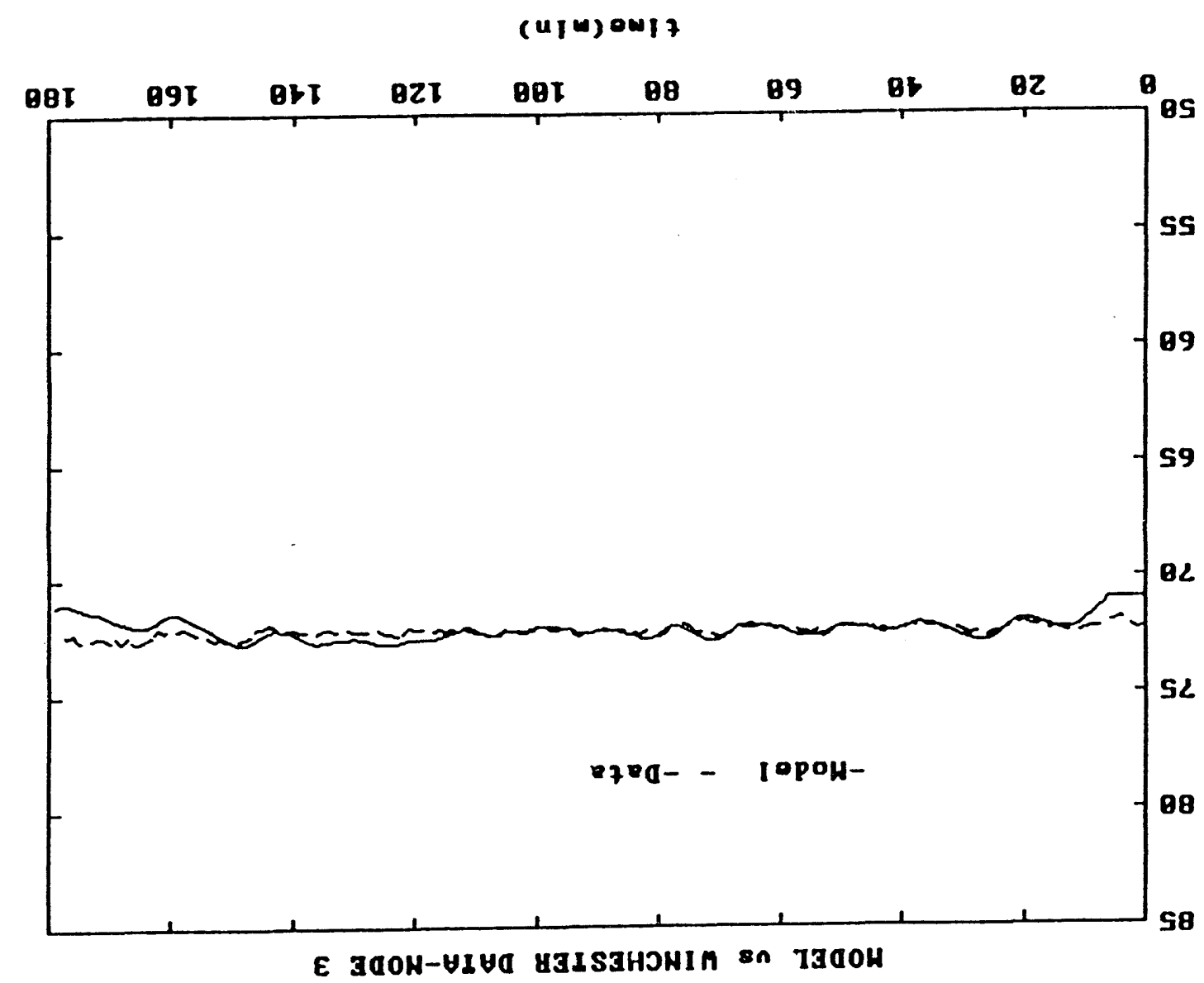




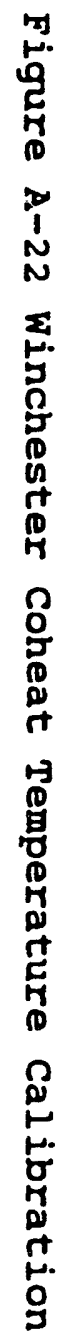

MODEL UE WIMCHESTER DATA-MODE 4

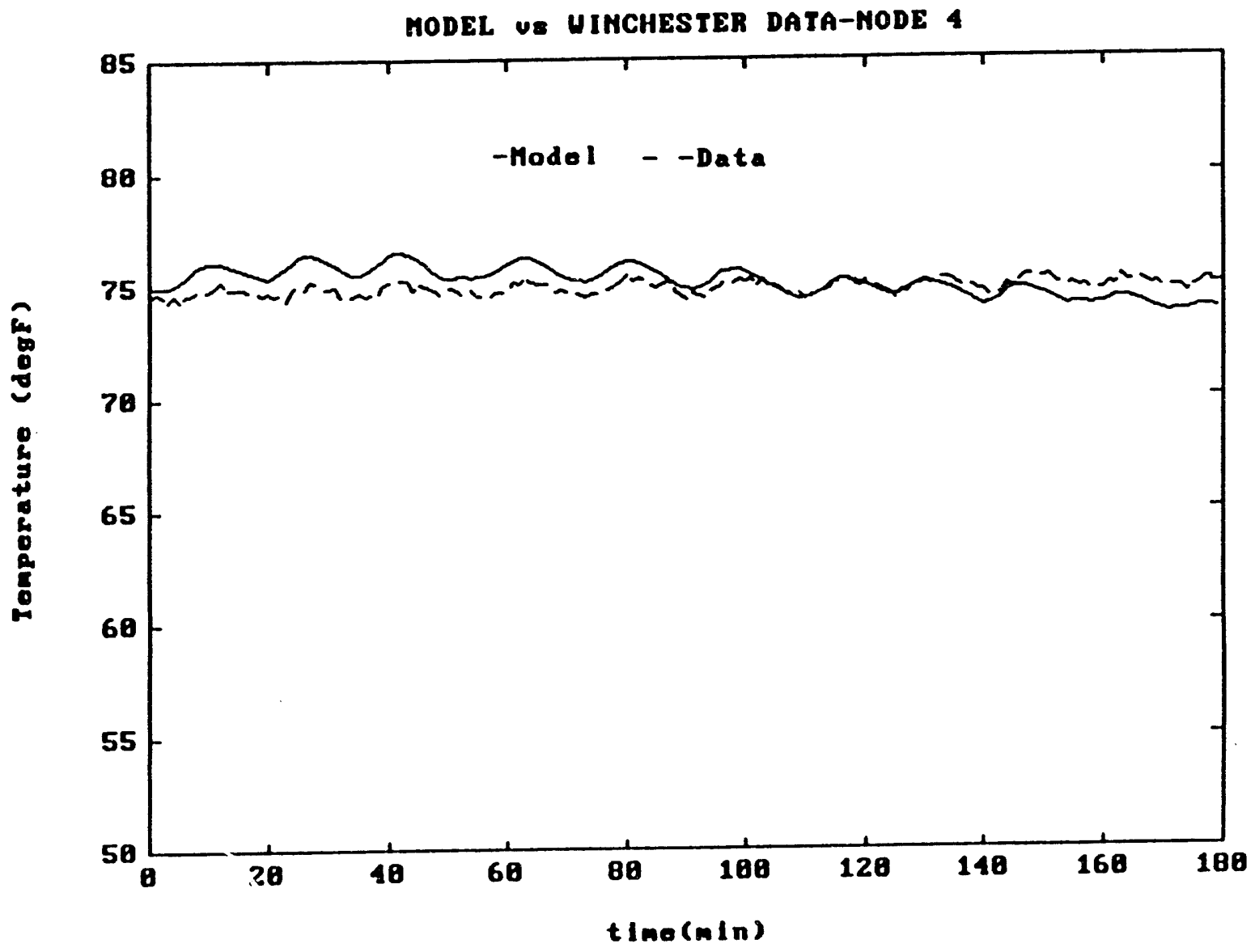




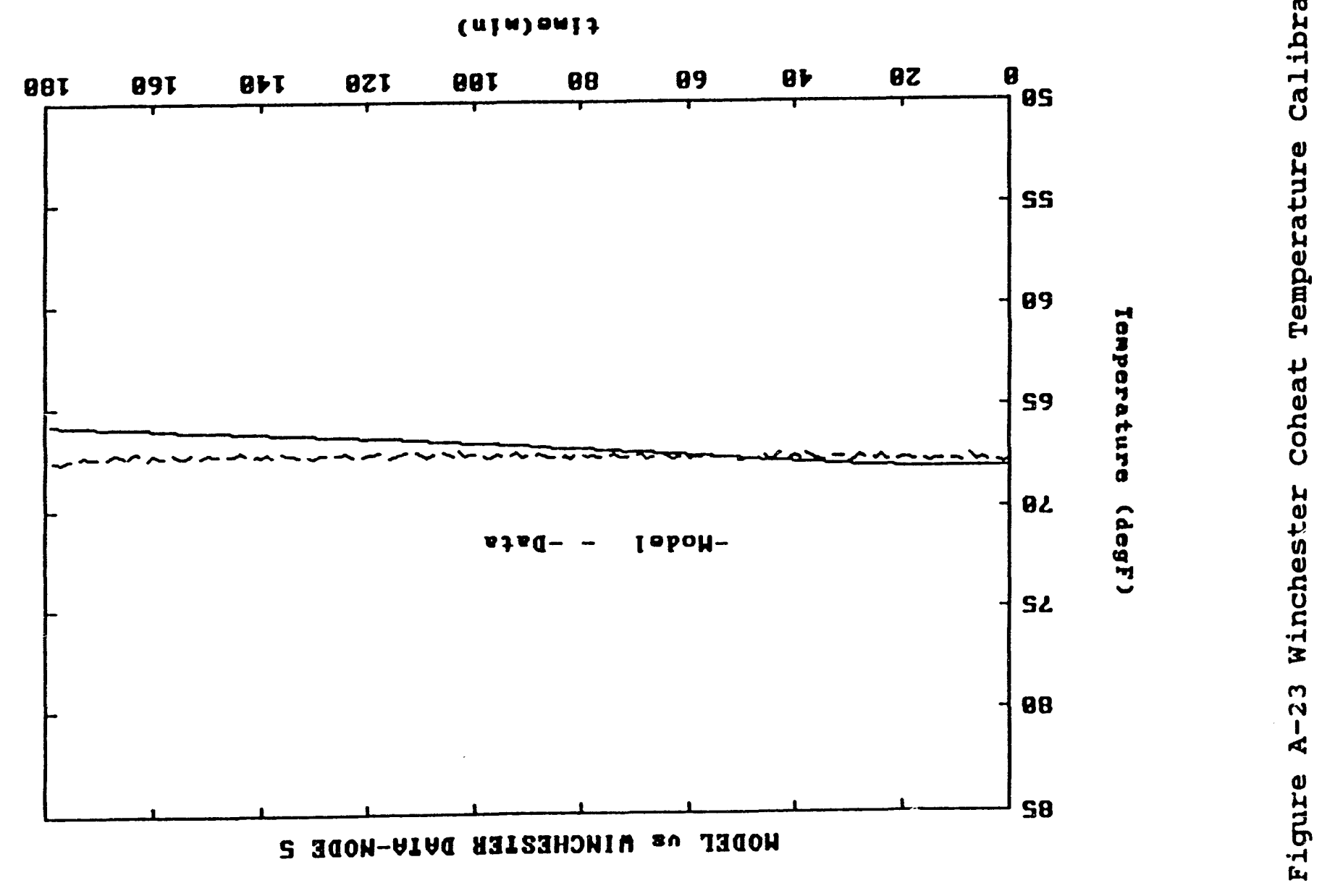




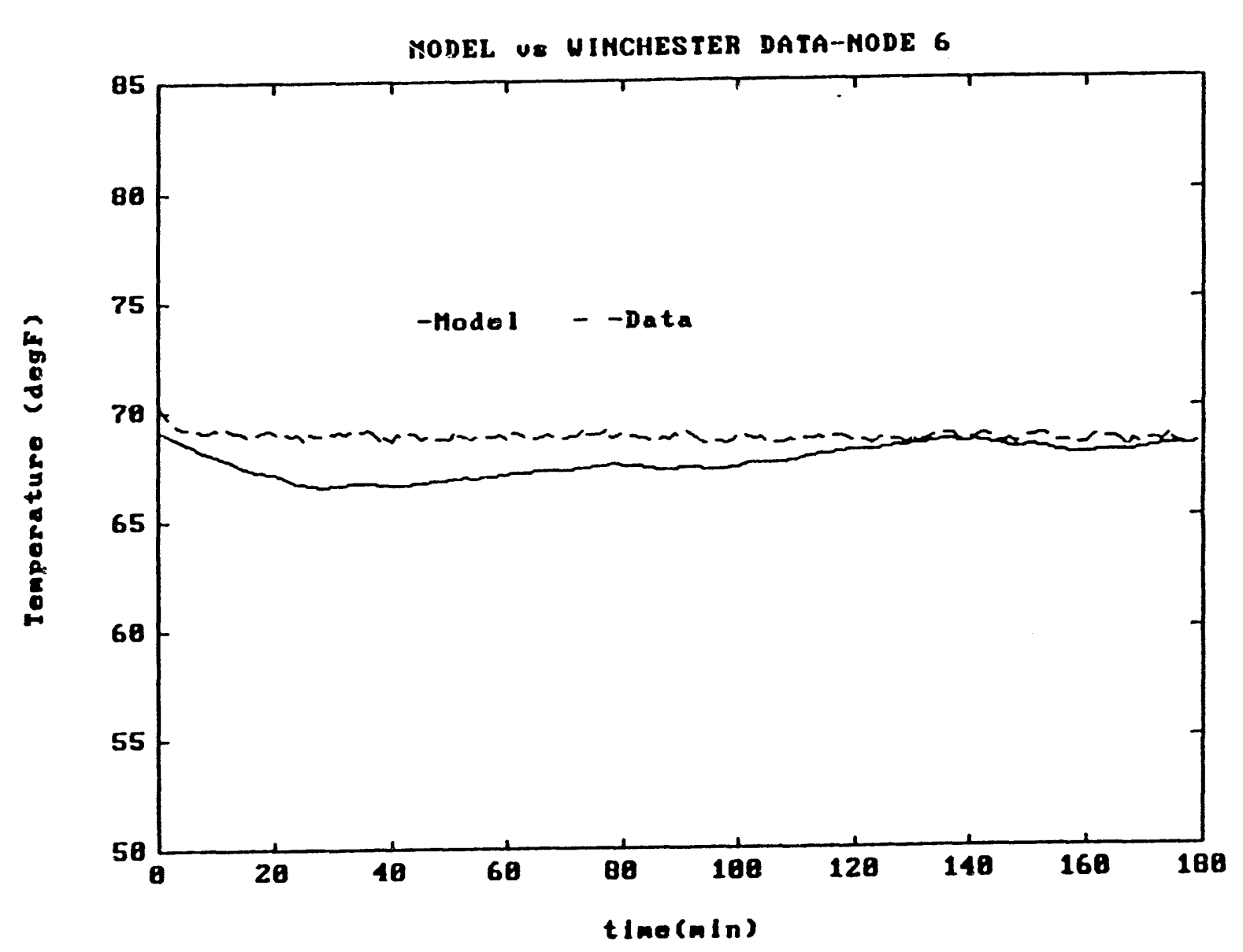




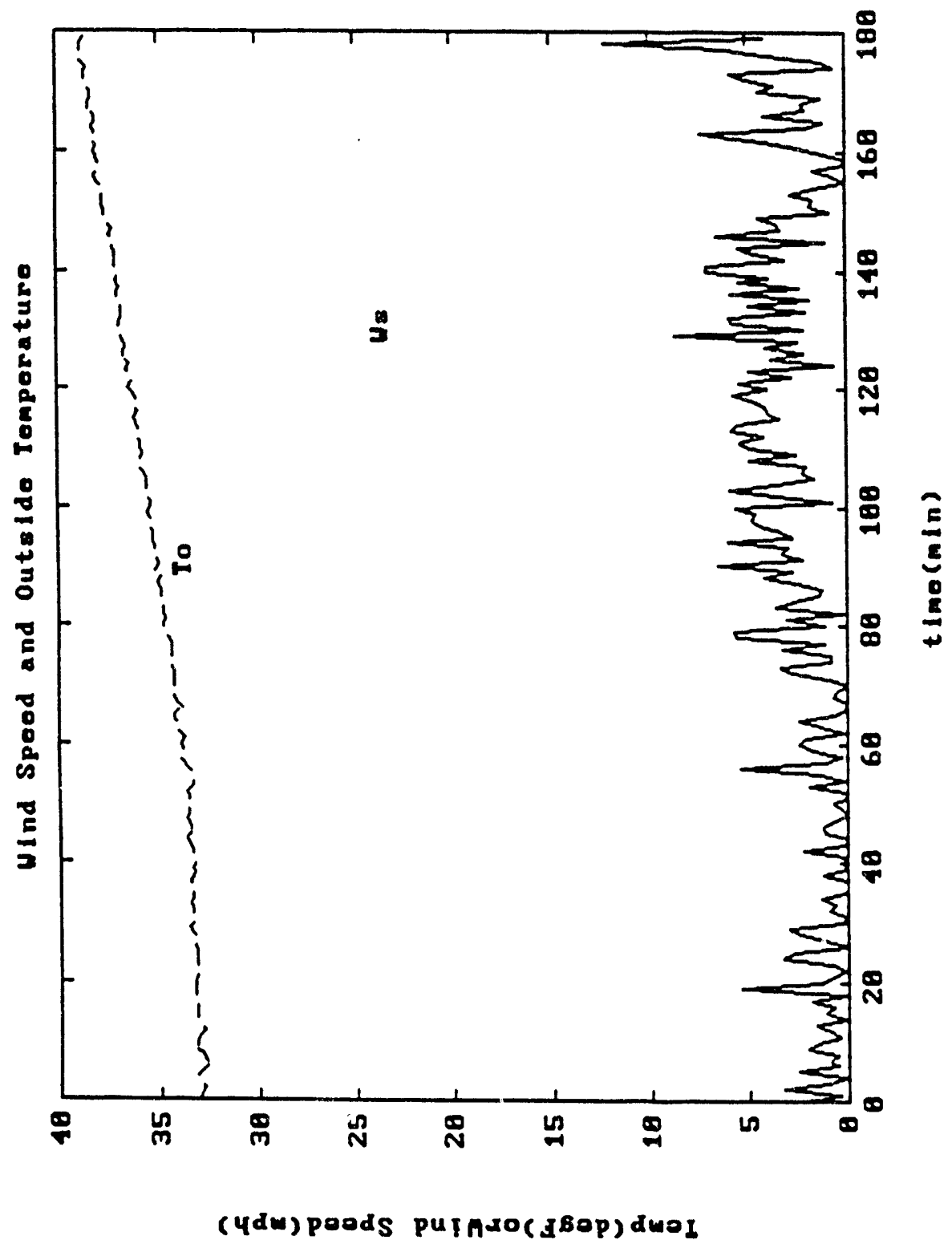

Figure A-25 Winchester Coheat Temperature Calibration D-104 
WINCHESTER ENERGY BALANCE CALIBRATION

D-105 


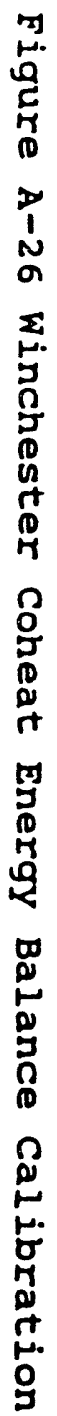

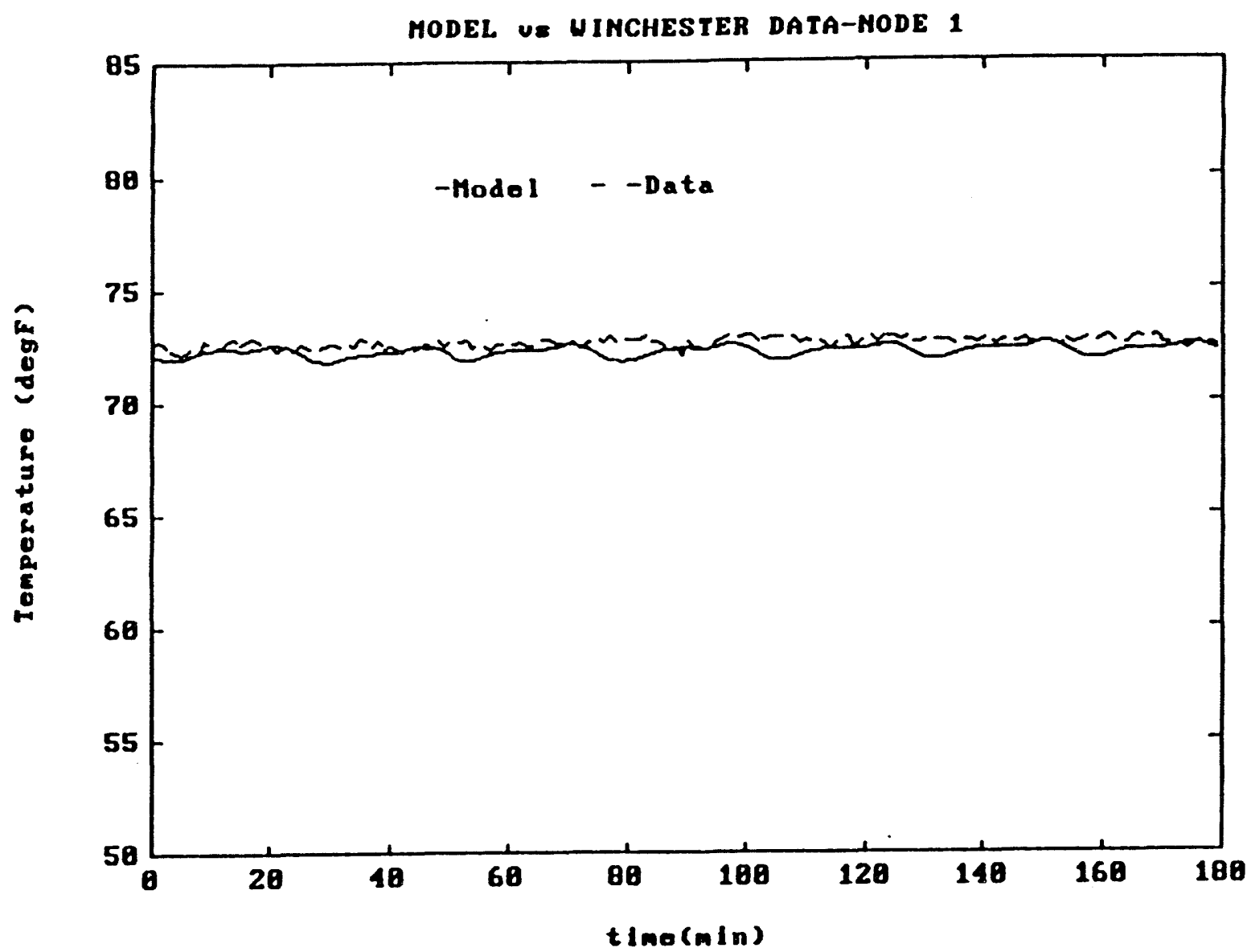




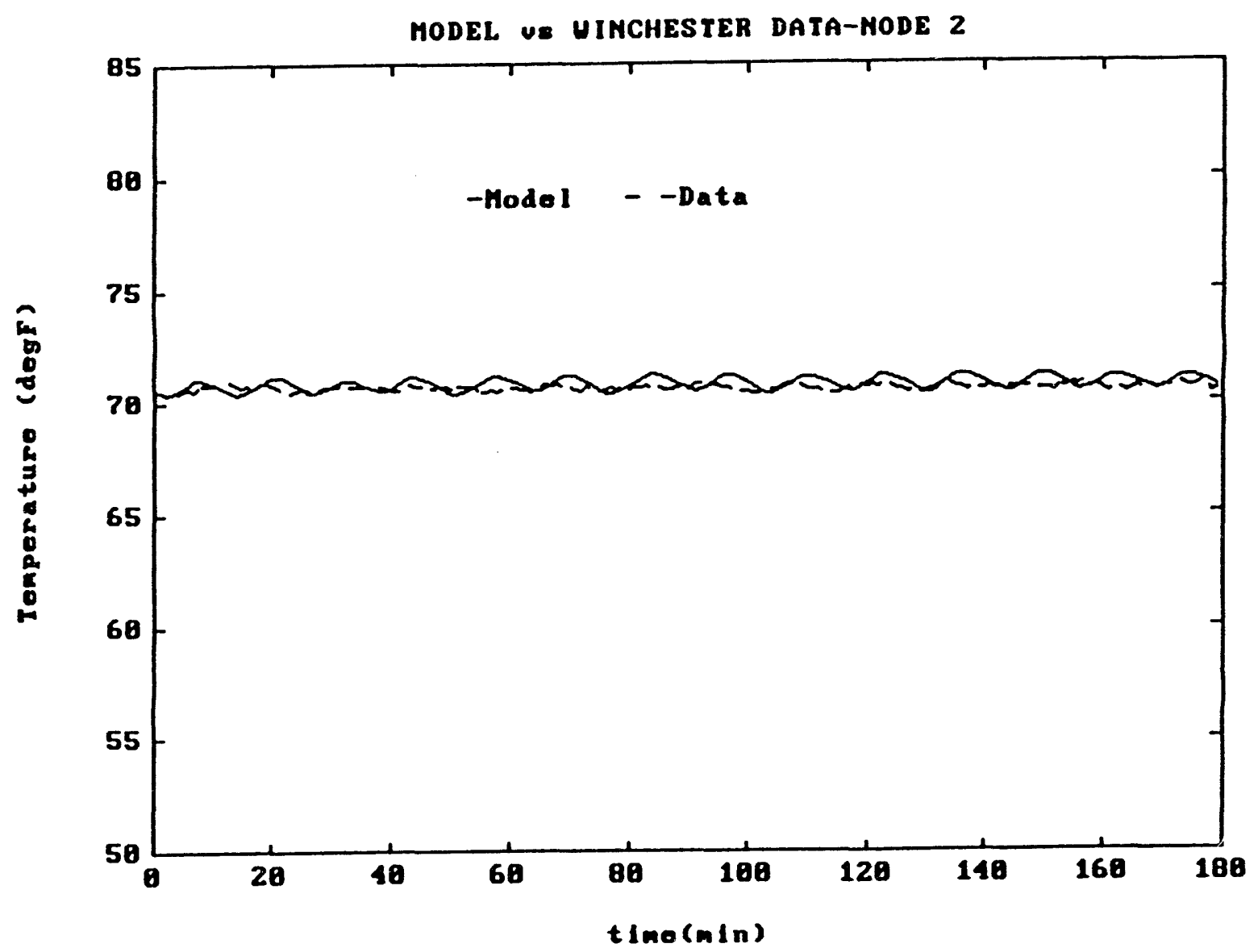




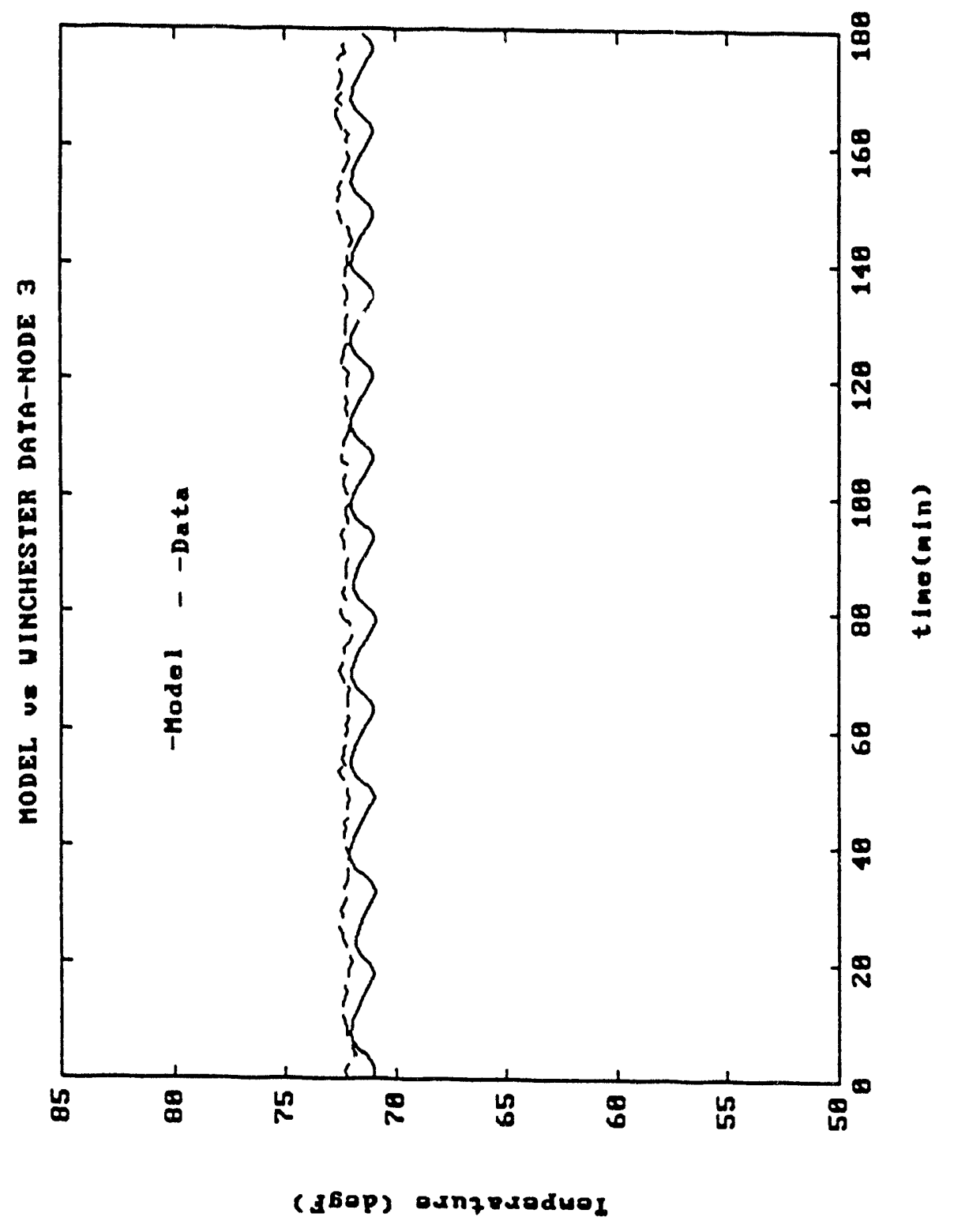

Figure A-28 Winchester Coheat Energy Balance Calibration 


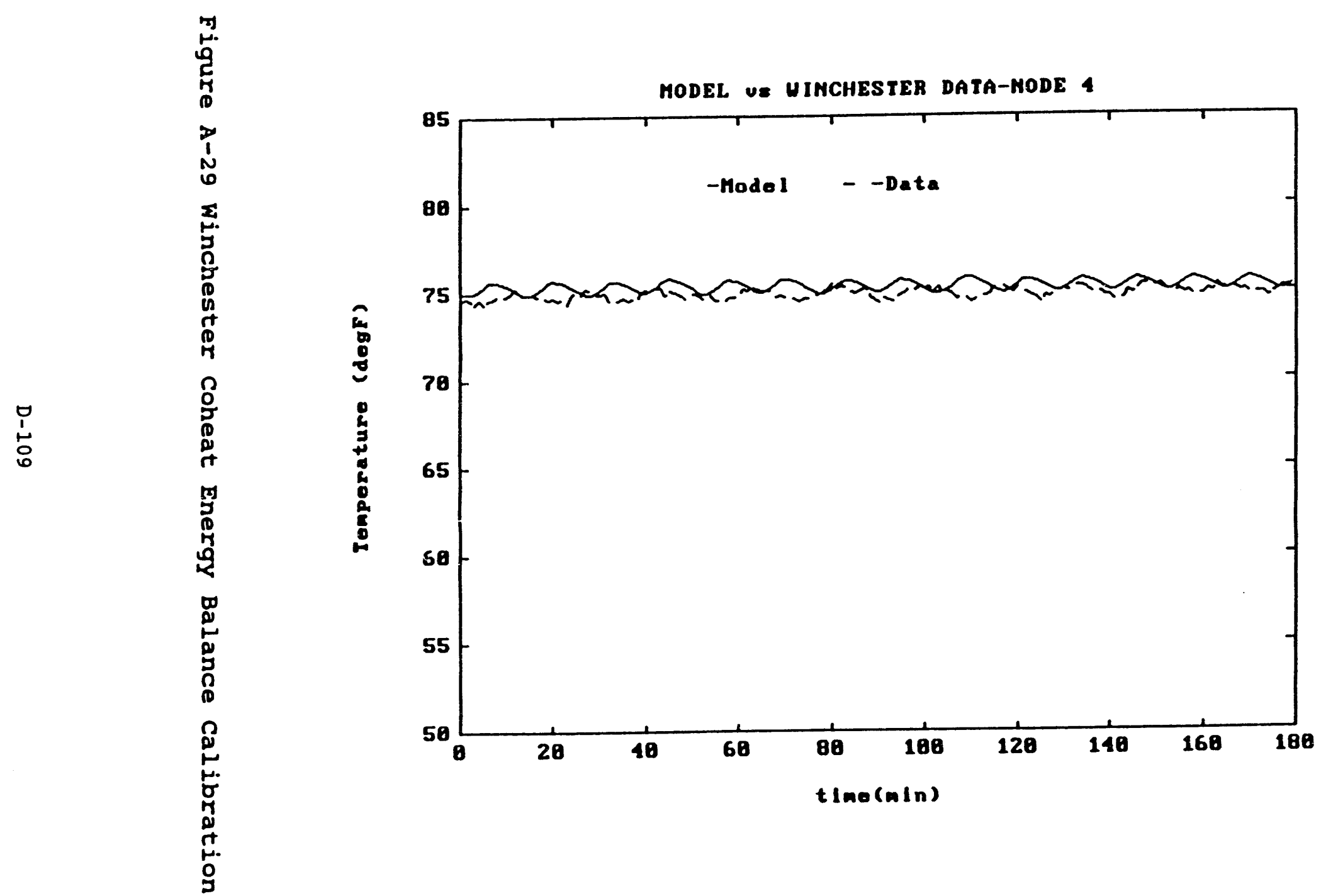




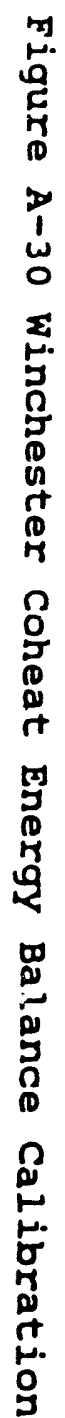

MODEL US UIMCHESTER DATA-MODE 5

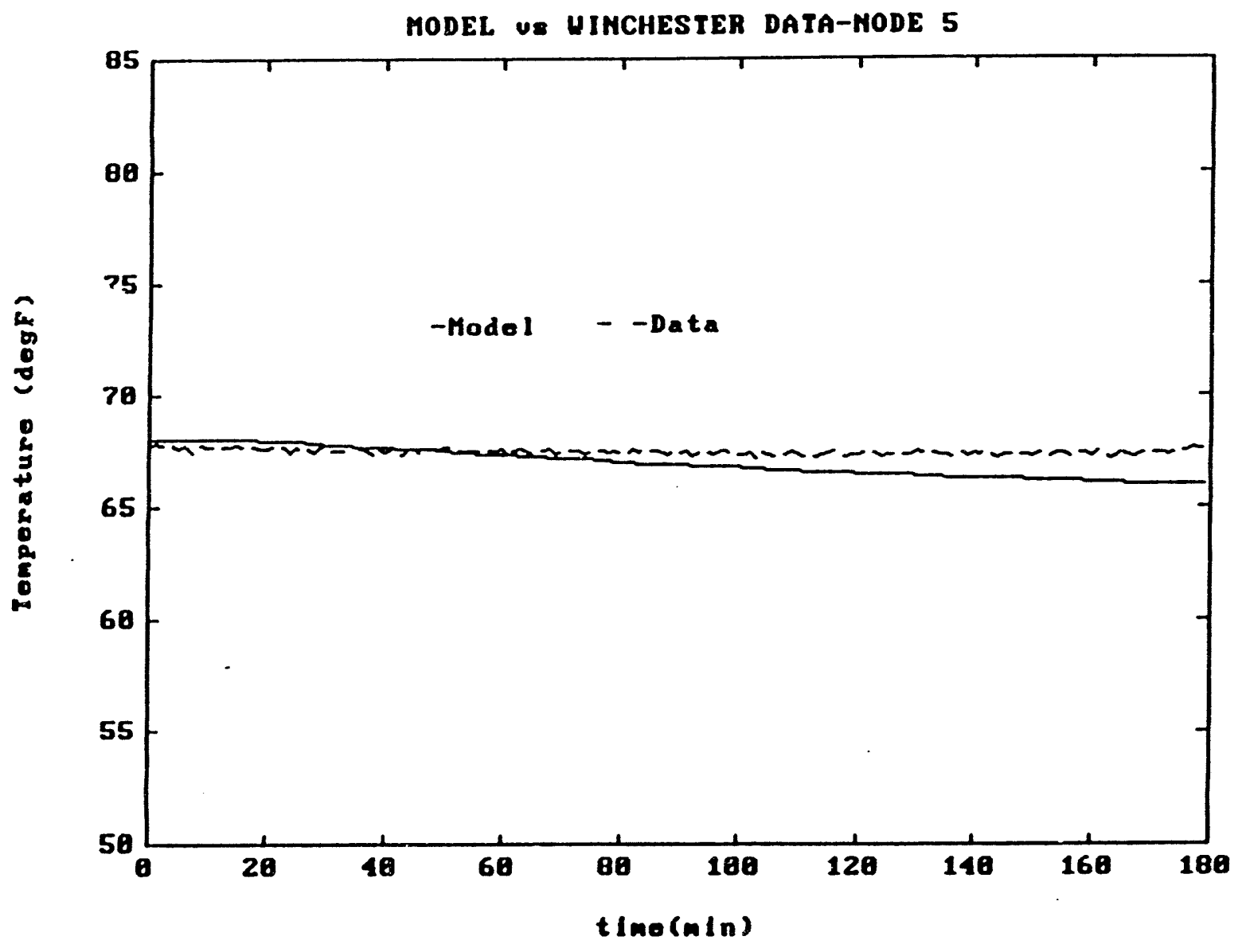




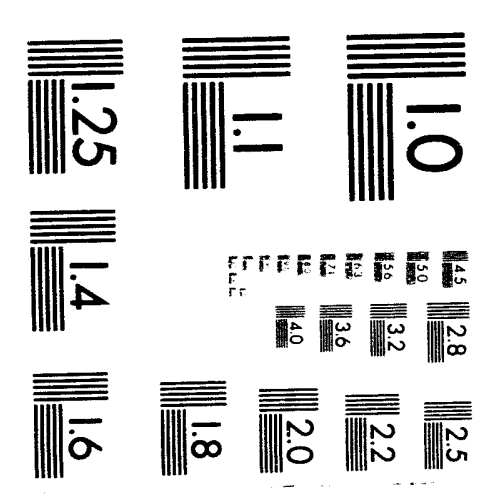



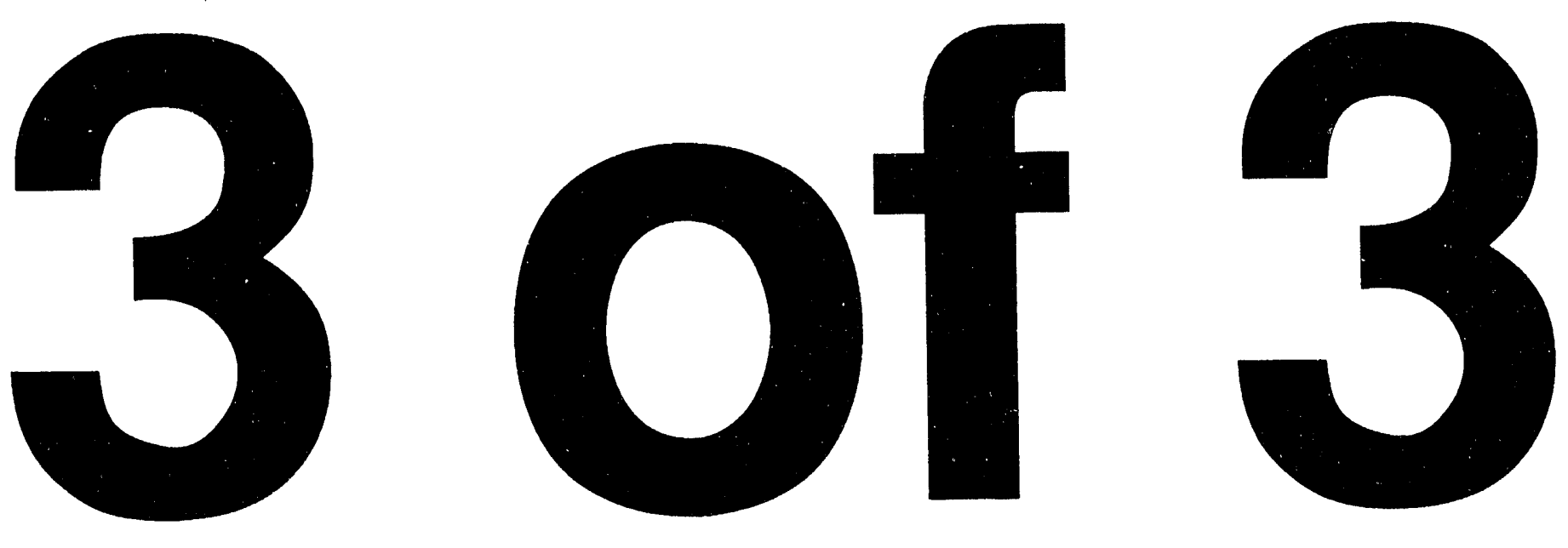


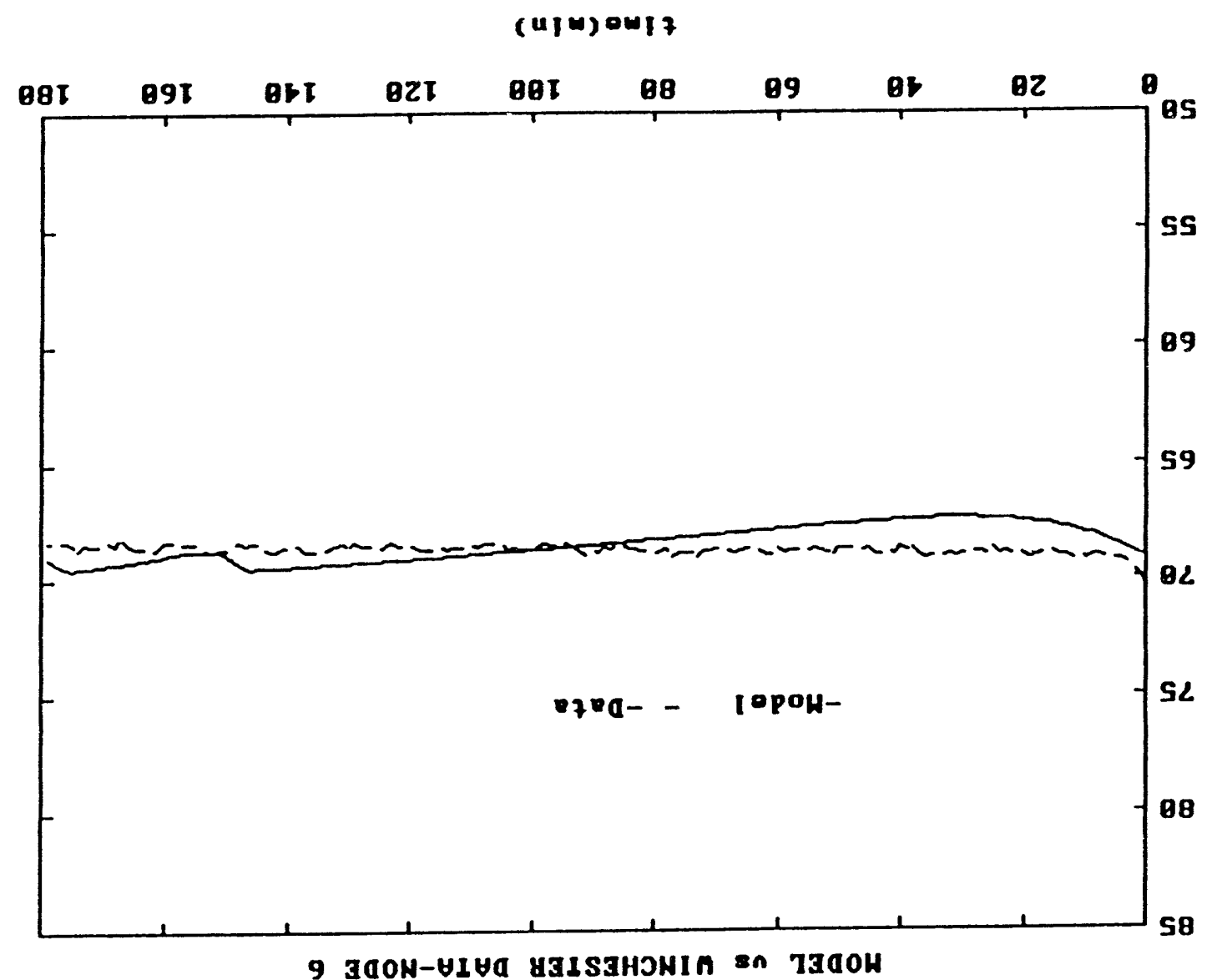

$\vec{I}$ 
WINCHESTER FURNACE DISTRIBUTION

AND

EFFICIENCY CALIBRATION 


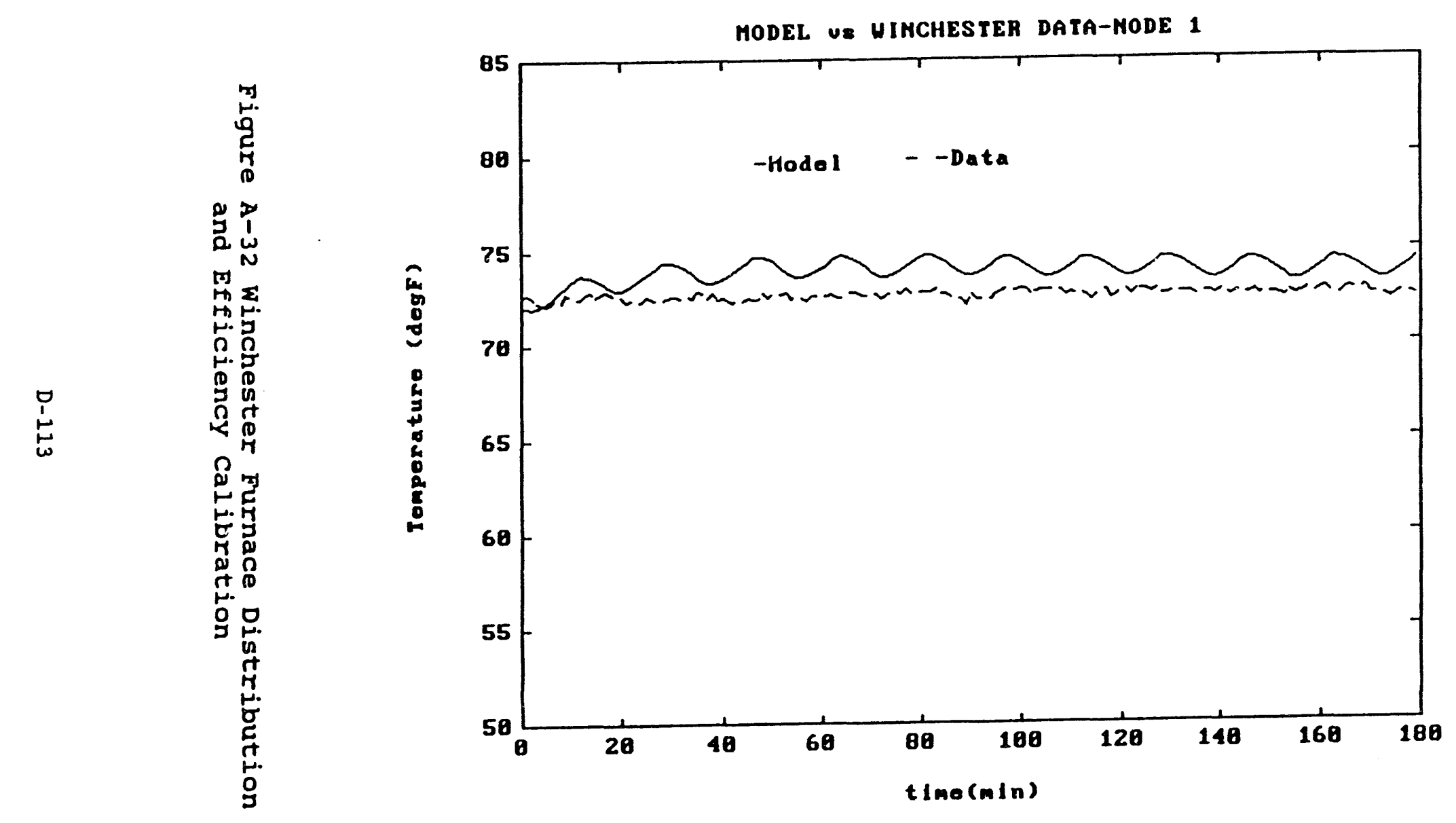




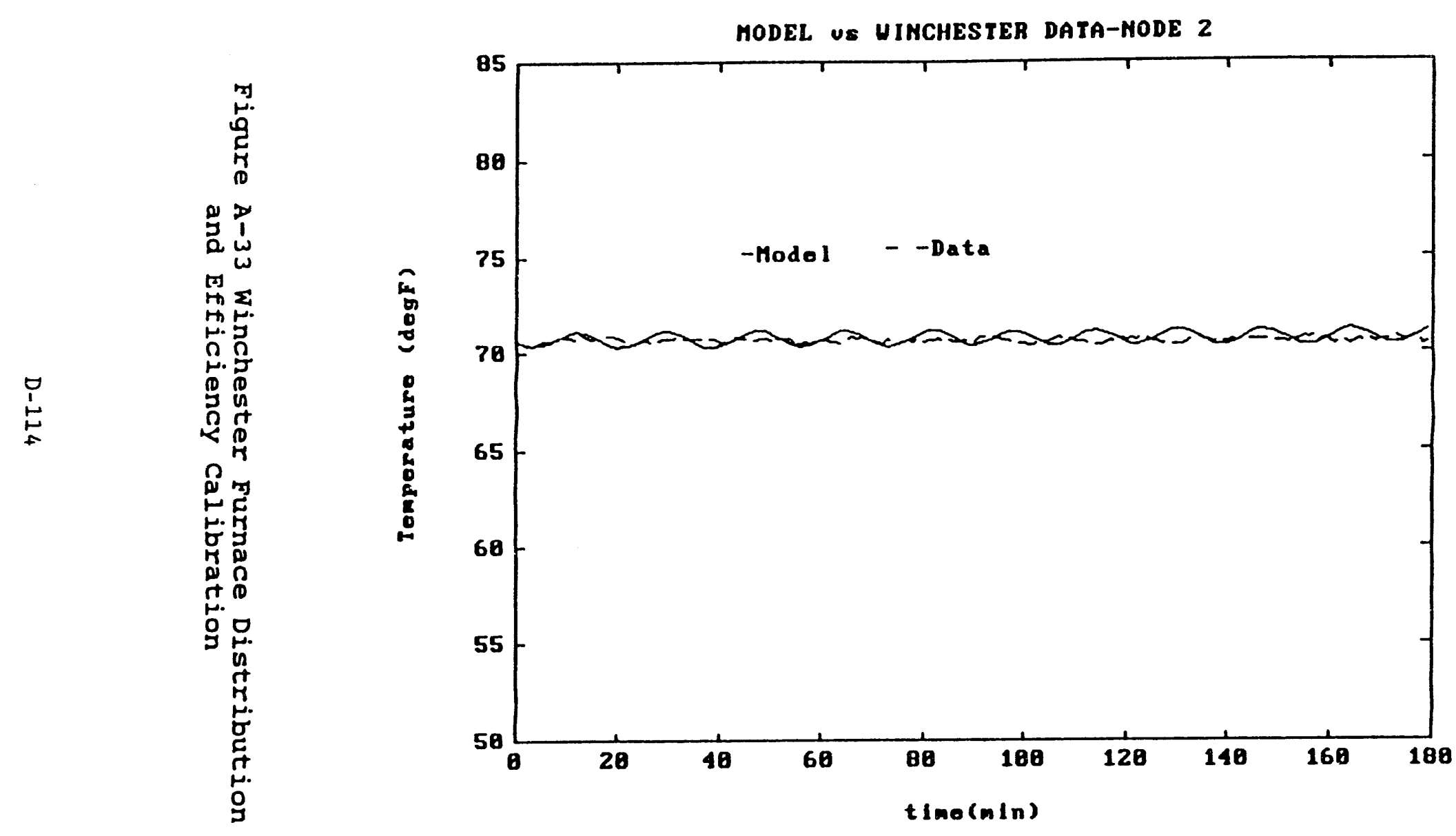




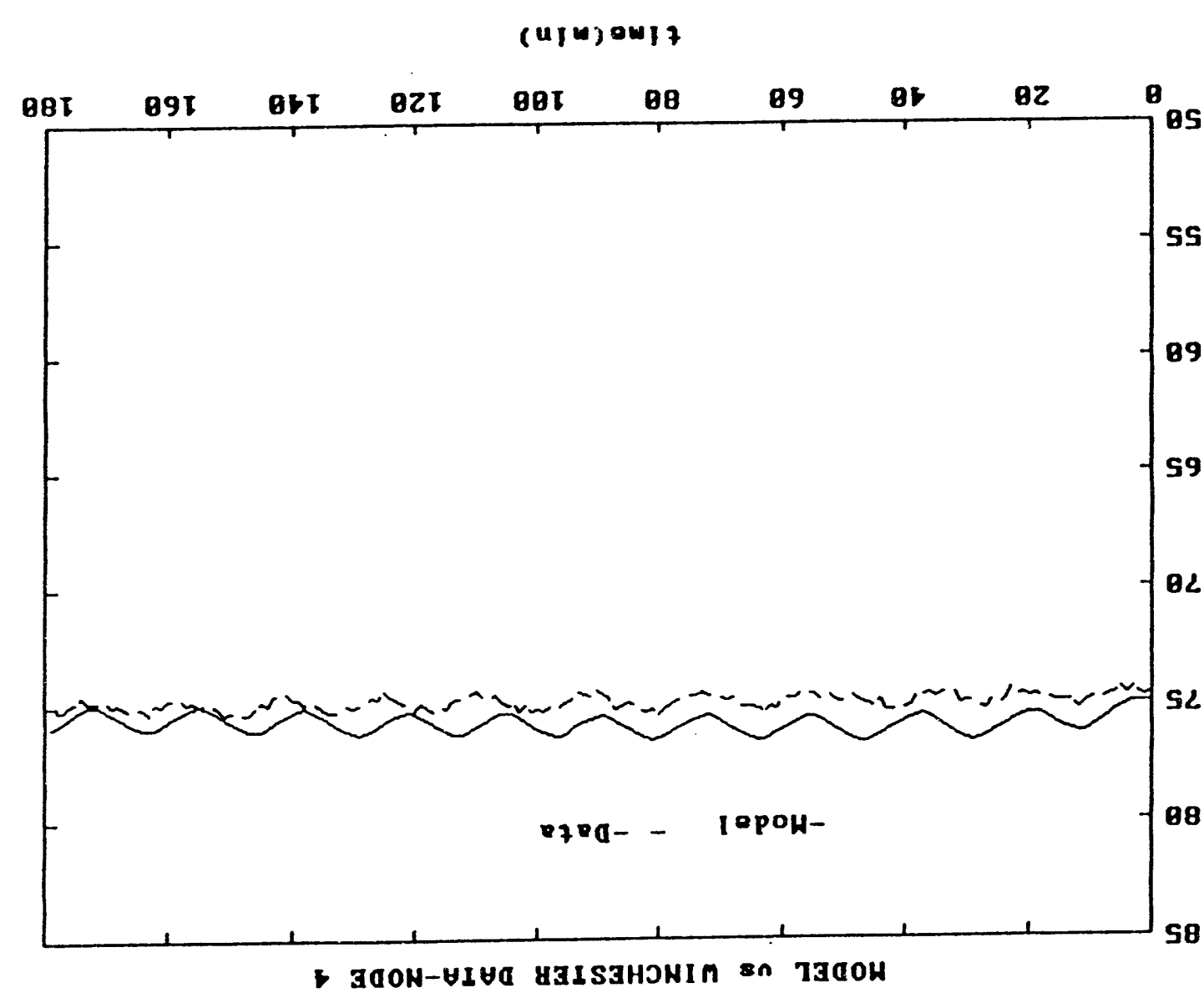




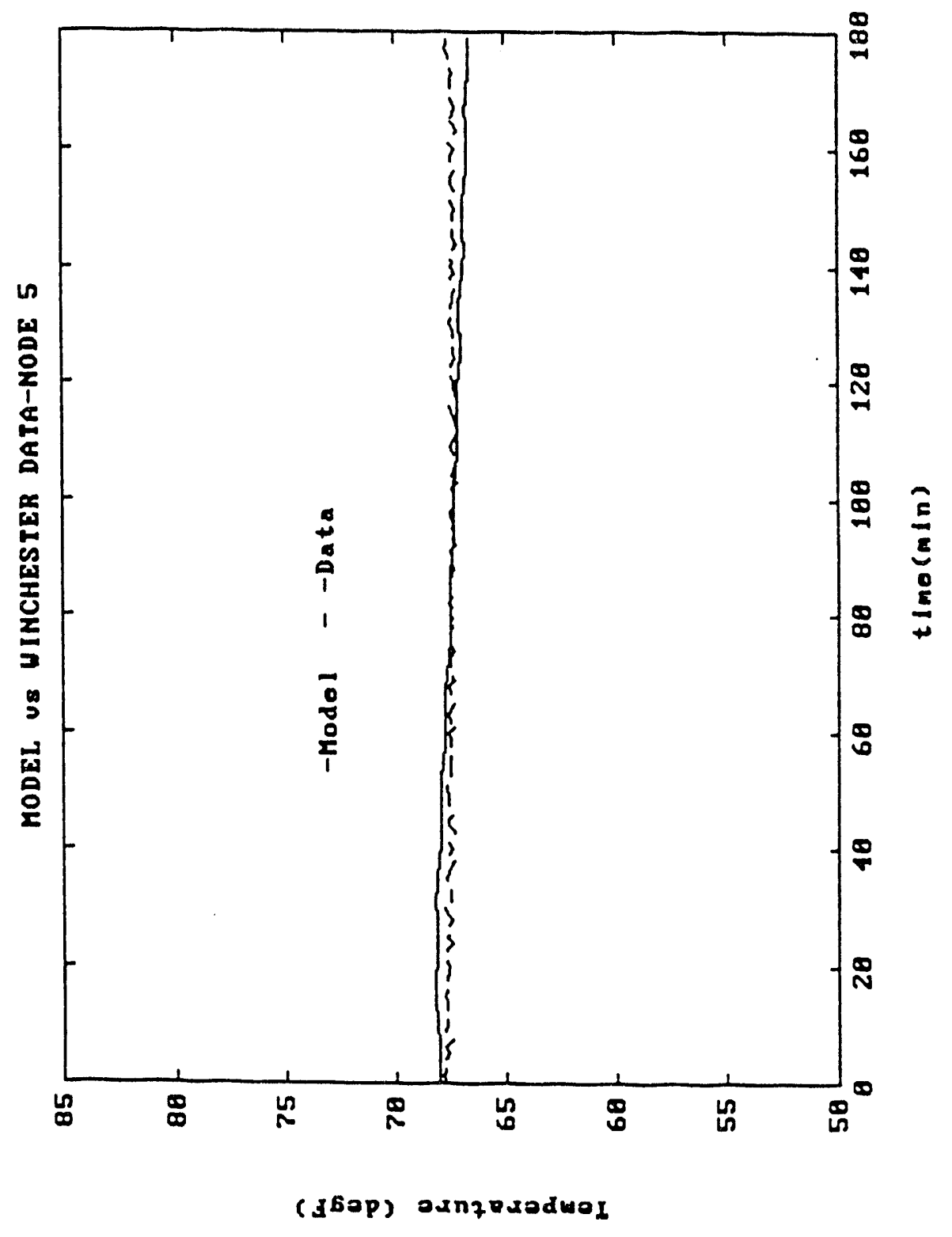

Figure A-35 Winchester Furnace Distribution and Efficiency Calibration 


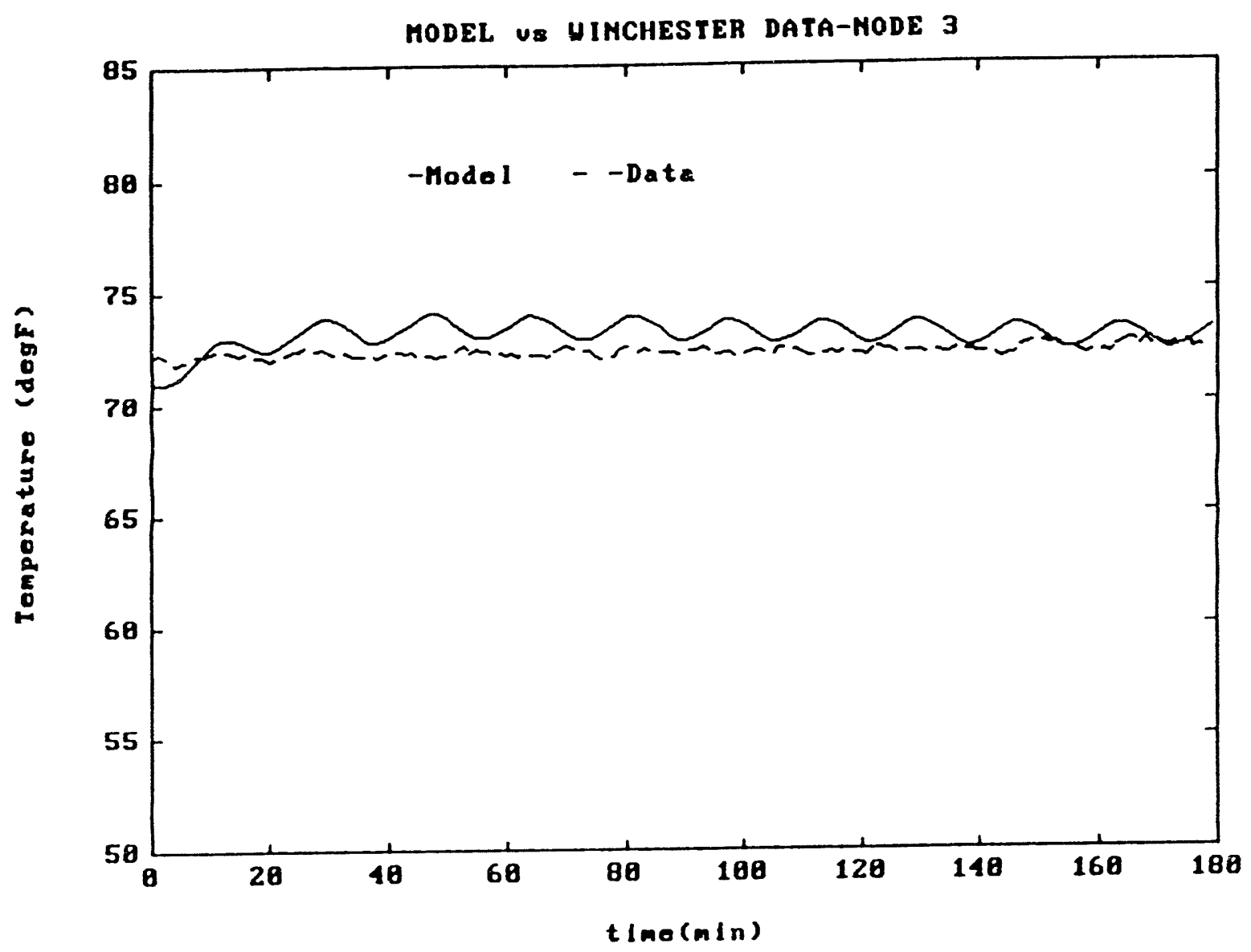




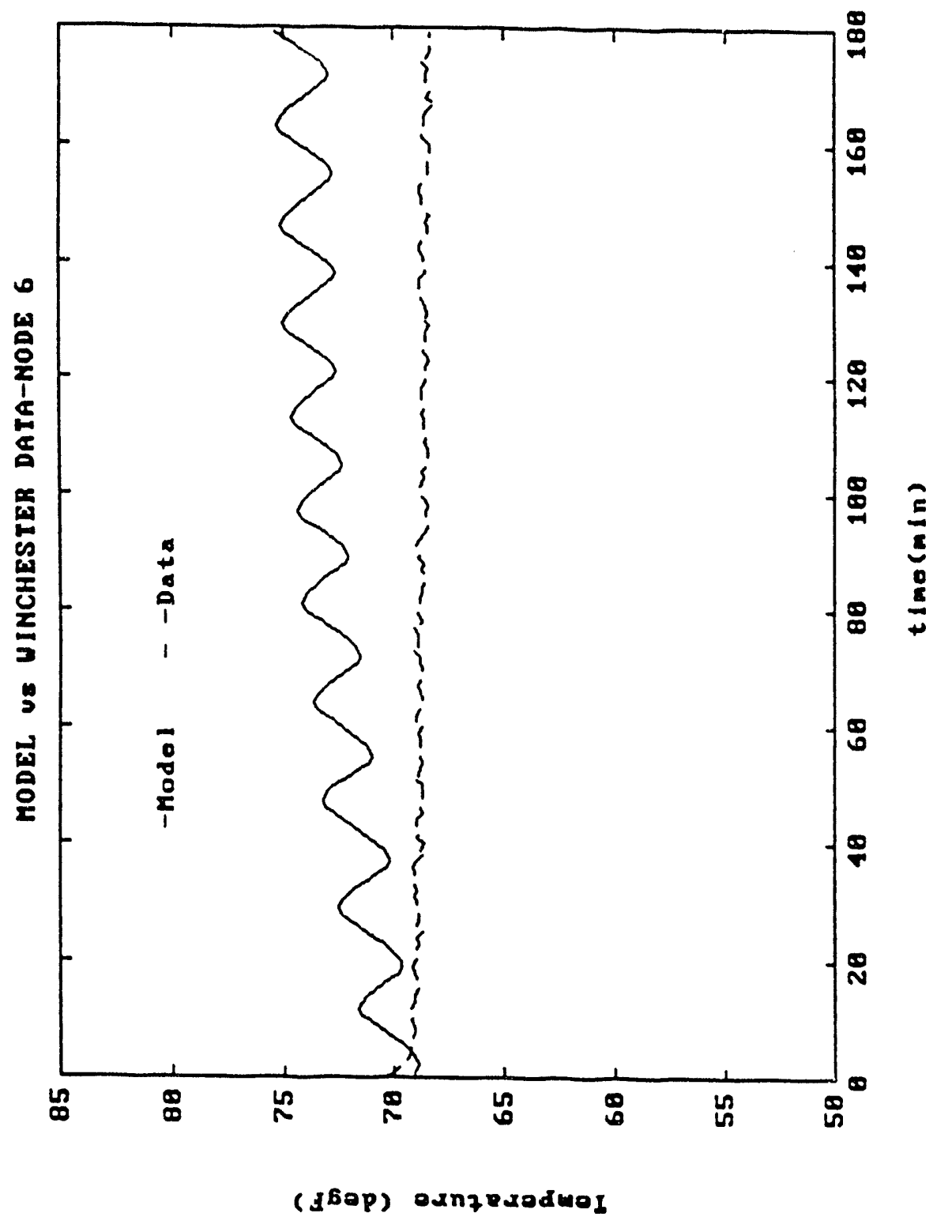

Figure A-37 Winchester Furnace Distribution and Efficiency Calibration 

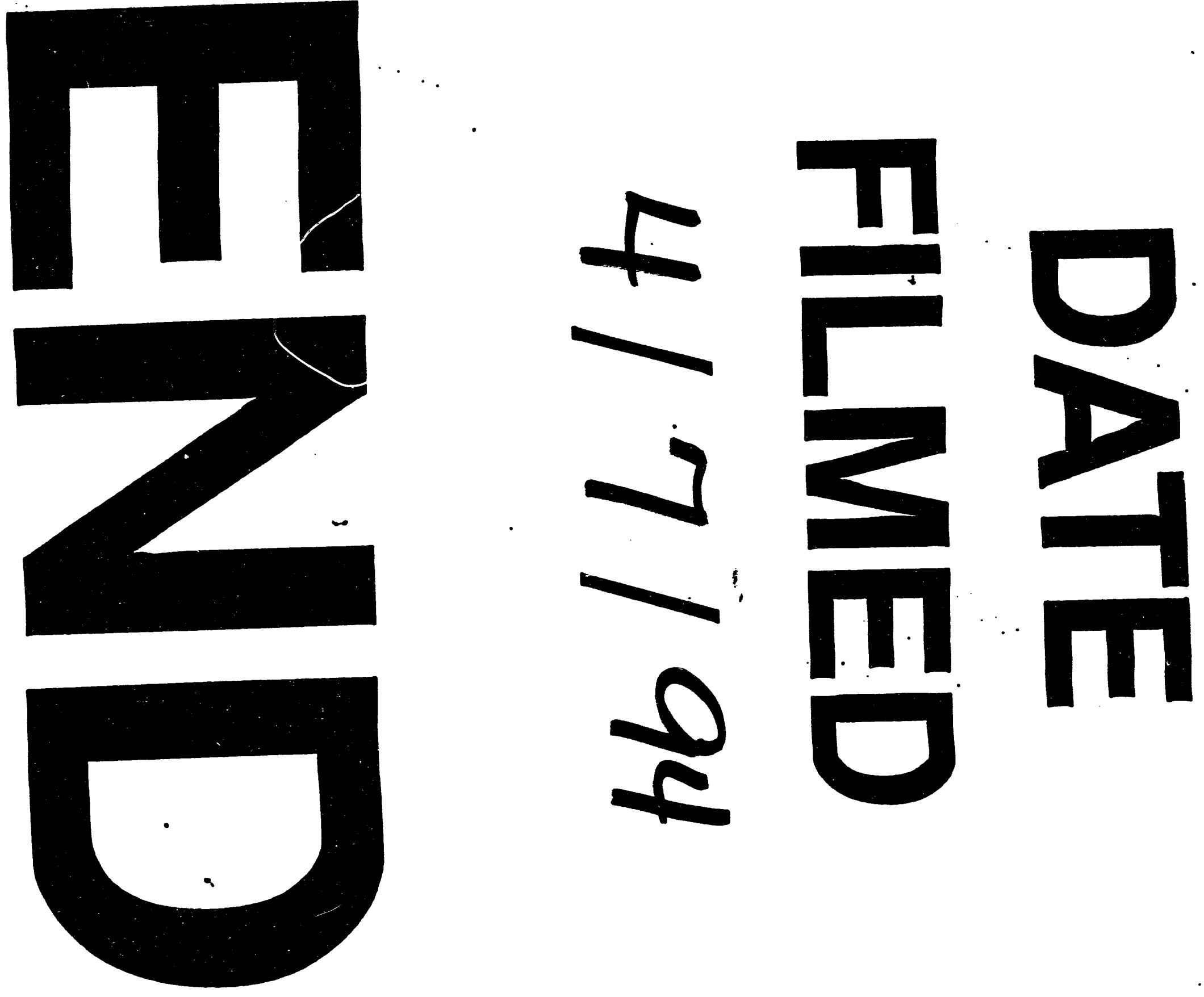
